Florida International University FIU Digital Commons

$10-5-2011$

\title{
Proton Nuclear Magnetic Resonance Investigation of the Native and Modified Active Site Structure of Heme Proteins
}

Zhonghua Wang

Florida International University, zwang003@fiu.edu

DOI: $10.25148 /$ etd.FI11120706

Follow this and additional works at: https://digitalcommons.fiu.edu/etd

\section{Recommended Citation}

Wang, Zhonghua, "Proton Nuclear Magnetic Resonance Investigation of the Native and Modified Active Site Structure of Heme Proteins" (2011). FIU Electronic Theses and Dissertations. 513.

https://digitalcommons.fiu.edu/etd/513 


\title{
FLORIDA INTERNATIONAL UNIVERSITY
}

\author{
Miami, Florida
}

PROTON NUCLEAR MAGNETIC RESONANCE INVESTIGATION OF THE NATIVE AND MODIFIED ACTIVE SITE STRUCTURE OF HEME PROTEINS

A dissertation submitted in partial fulfillment of the

requirements for the degree of

DOCTOR OF PHILOSOPHY

in

CHEMISTRY

by

Zhonghua Wang 
To: Dean Kenneth Furton

College of Arts and Sciences

This dissertation, written by Zhonghua Wang, and entitled Proton Nuclear Magnetic Resonance Investigation of the Native and Modified Active Site Structure of Heme Proteins, having been approved in respect to style and intellectual content, is referred to you for judgment.

We have read this dissertation and recommend that it be approved.

Watson Lees

Fenfei Leng

Kathleen Rein

Lidia Kos

Xiaotang Wang, Major Professor

Date of Defense: October 5, 2011

The dissertation of Zhonghua Wang is approved.

$\begin{array}{r}\begin{array}{r}\text { Dean Kenneth Furton } \\ \text { College of Arts and Sciences }\end{array} \\ \hline \begin{array}{r}\text { Dean Lakshmi Reddi } \\ \text { University Graduate School }\end{array}\end{array}$

Florida International University, 2011 
(C) Copyright 2011 by Zhonghua Wang

All rights reserved. 


\section{DEDICATION}

I dedicate this dissertation to my wife, Taiyi Chen, my mother, Fenglan Jiang and my

father, You Wang. Without their understanding, encouragement, support, and love, the completion of this work would not have been possible. 


\section{ACKNOWLEDGMENTS}

I am deeply grateful to many people that have helped me during my delightful and rewarding graduate study at Florida International University in the past few years. First, I would like to thank my research advisor, Dr. Xiaotang Wang, who has been not only an intelligent and respectable mentor, but also a close and thoughtful friend to me. His patience, guidance, and encouragement strongly supported me through my graduate studies and have offered me a great foundation to build a career in science. I would also like to thank all my committee members, Dr. Watson Lees, Dr. Fenfei Leng, Dr. Kathleen Rein and Dr. Lidia Kos, for all of their constructive advice and professional assistance. Thanks to all the members of Dr. Wang's group, especially Wei Lin, Zheng Wang, Lin Jiang, Hui Tian, Rui Zhang, Hua Ling, Dr. Hedong Bian, and Dr. Yuchang Jiang, for all of the inspiring discussions, selfless help, and for creation of a pleasant lab environment. I would like to extend my appreciation to Yali Hsu and Dr. Yaru Song for their enthusiastic help and careful maintenance of the NMR and MS instruments. Special thanks go to Dr. Palmer Graves and Dr. Uma Swamy for all their kindness help and guidance to teach me how to be a good teaching assistant. I would like to especially acknowledge Dr. Chengxiao Zhang and Dr. Peng Lu for all their care, support and encouragement to me during the years. I also want to thank all of my family and friends for their continuous support, understanding, and encouragement. Finally, I would like to thank the Department of Chemistry and Biochemistry, Florida International University, the National Institutes of Health, and the National Science Foundation for the assistantship support and the funding support during my graduate studies. 


\begin{abstract}
OF THE DISSERTATION
PROTON NUCLEAR MAGNETIC RESONANCE INVESTIGATION OF THE

NATIVE AND MODIFIED ACTIVE SITE STRUCTURE OF HEME PROTEINS
\end{abstract}

by

Zhonghua Wang

Florida International University, 2011

Miami, Florida

\title{
Professor Xiaotang Wang, Major Professor
}

Hemoproteins are a very important class of enzymes in nature sharing the essentially same prosthetic group, heme, and are good models for exploring the relationship between protein structure and function. Three important hemoproteins, chloroperoxidase (CPO), horseradish peroxidase (HRP), and cytochrome P450cam (P450cam), have been extensively studied as archetypes for the relationship between structure and function. In this study, a series of $1 \mathrm{D}$ and 2D NMR experiments were successfully conducted to contribute to the structural studies of these hemoproteins.

During the epoxidation of allylbenzene, $\mathrm{CPO}$ is converted to an inactive green species with the prosthetic heme modified by addition of the alkene plus an oxygen atom forming a five-membered chelate ring. Complete assignment of the NMR resonances of the modified porphyrin extracted and demetallated from green CPO unambiguously established the structure of this porphyrin as an $\mathrm{N}_{\mathrm{III}}$-alkylated product. A novel substrate binding motif of $\mathrm{CPO}$ was proposed from this concluded regiospecific $\mathrm{N}$-alkylation structure.

Soybean peroxidase (SBP) is considered as a more stable, more abundant and less expensive substitute of HRP for industrial applications. A NMR study of SBP using 1D 
and 2D NOE methods successfully established the active site structure of SBP and consequently fills in the blank of the SBP NMR study. All of the hyperfine shifts of the $\mathrm{SBP}-\mathrm{CN}^{-}$complex are unambiguously assigned together with most of the prosthetic heme and all proximal His 170 resonances identified. The active site structure of SBP revealed by this NMR study is in complete agreement with the recombinant SBP crystal structure and is highly similar to that of the HRP with minor differences.

The NMR study of paramagnetic P450cam had been greatly restricted for a long time. A combination of 2D NMR methods was used in this study for $\mathrm{P} 450$ cam- $\mathrm{CN}^{-}$complexes with and without camphor bound. The results lead to the first unequivocal assignments of all heme hyperfine-shifted signals, together with certain correlated diamagnetic resonances. The observed alternation of the assigned novel proximal cysteine $\beta-\mathrm{CH}_{2}$ resonances induced by camphor binding indicated a conformational change near the proximal side. 


\section{TABLE OF CONTENTS}

CHAPTER

PAGE

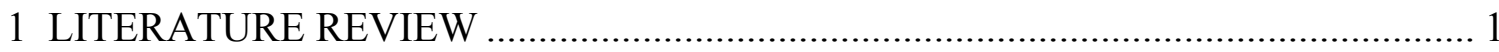

1.1 Introduction to Hemoproteins ............................................................................ 1

1.2 Heme-Thiolate Proteins .................................................................................. 5

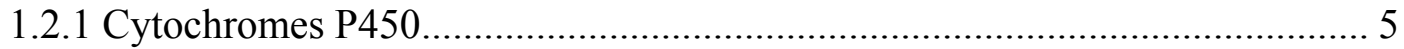

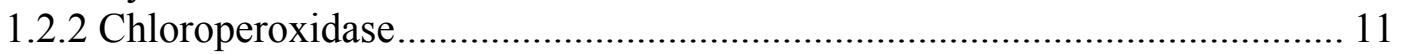

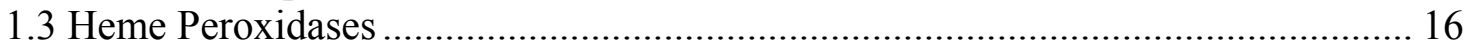

1.3.1 Horseradish Peroxidase ........................................................................ 17

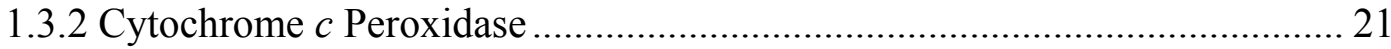

1.3.3 Soybean Peroxidase ............................................................................ 22

1.4 Nuclear Magnetic Resonance of Hemoproteins..................................................... 24

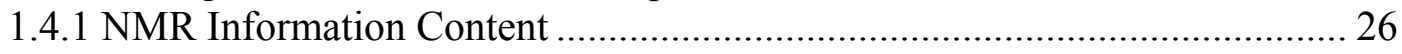

1.4.2 Temperature Dependence of Hyperfine Shifts ……………………….......... 27

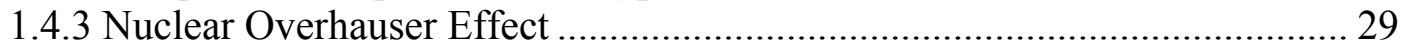

1.5.2 Low-Spin Cynide Complex of Hemoprotein ………………….................. 30

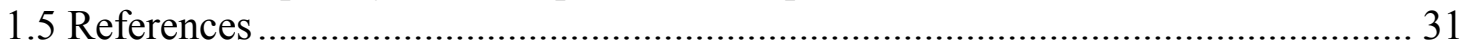

2 ISOLATION AND STRUCTURE DETERMINATION OF A NOVEL HEME IN ALLYLBENZENE-MODIFIED CHLOROPEROXIDASE ……………………........ 44

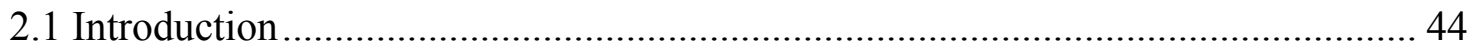

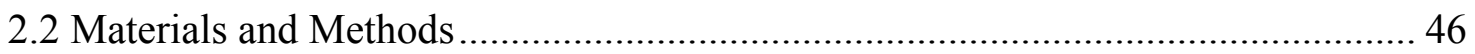

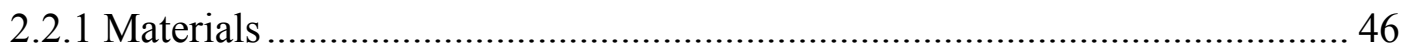

2.2.2 Growth of Mold and Preparation of CPO.................................................. 46

2.2.3 Preparation, Extraction and Purification of Allylbenzene-Modified Heme.. 47

2.2.4 Spectroscopic Characterization of Green Heme............................................ 47

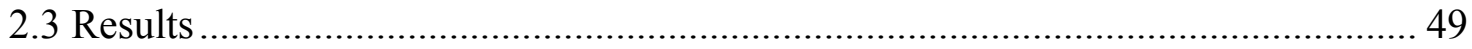

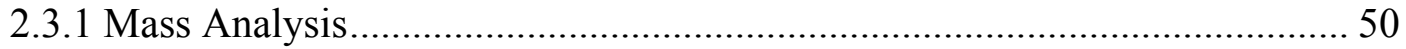

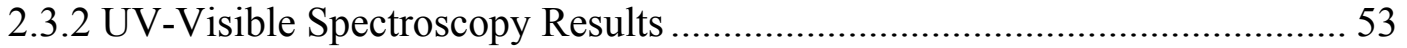

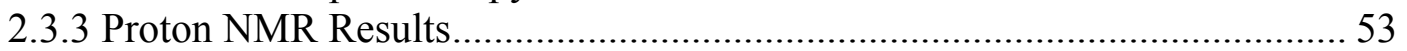

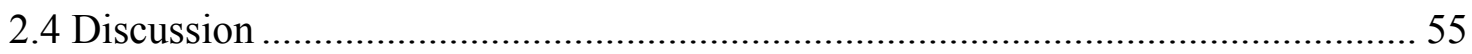

2.4.1 Assighment of the Porphyrin Resonances of $N$-ABPP .................................. 55

2.4.2 Assignment of the Linking Group Resonances of $N$-ABPP.......................... 62

2.4.3 Identification of the Predominant Regioisomer of $N$-ABPP .......................... 63

2.4.4 Mechanism of Epoxidation and Substrate Binding of CPO Revealed by the

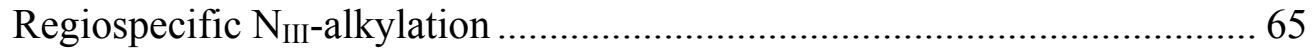

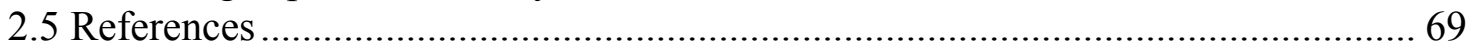

3 THE ACTIVE SITE STRUCTURE OF SOYBEAN PEROXIDASE AS PROBED BY 1D AND 2D NUCLEAR OVERHAUSER EFFECT ………................................. 76

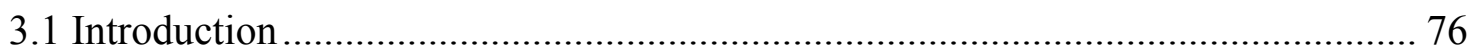

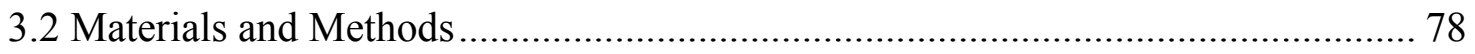

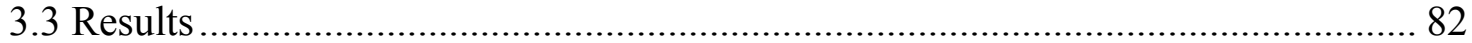

3.3.1 Proton NMR of the Native Ferric High-Spin SBP ...................................... 82 
3.3.2 Proton NMR of the Cyanide Adduct of Native SBP .................................... 83

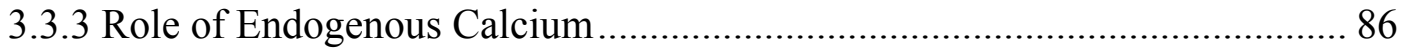

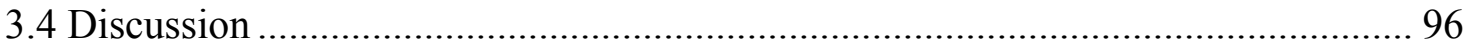

3.4.1 Assignment of the Hyperfine-Shifted Resonances for Native SBP ............. 96

3.4.2 Hyperfine-Shifted Heme Resonances Assignments for the

SBP-CN Complex ................................................................................ 98

3.4.3 Distal and Proximal Histidine Assignments for the SBP-CN ${ }^{-}$Complex..... 102

3.4.4 Distal Arginine Assignments for the SBP-CN Complex............................ 104

3.4.5 2D NOESY and COSY Results for the SBP-CN Complex....................... 105

3.4.6 Attempted Calcium Removal and Reconstitution ....................................... 106

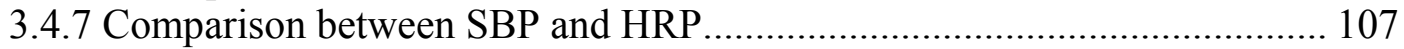

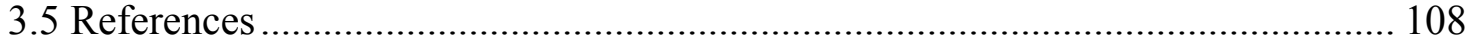

$4{ }^{1}$ H-NMR STUDY OF PARAMAGNETIC CYTOCHROME P450CAM-CYANIDE COMPLEXES: ASSIGNMENT OF HYPERFINE-SHIFTED HEME RESONANCES

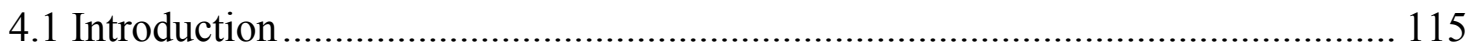

4.2 Materials and Methods ................................................................................. 118

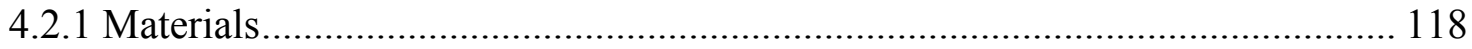

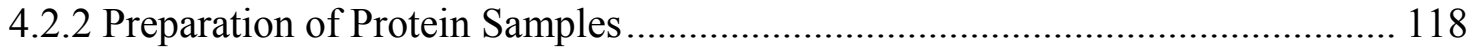

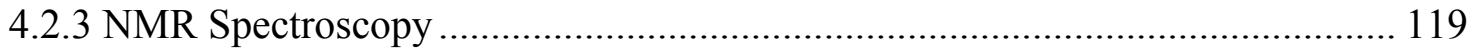

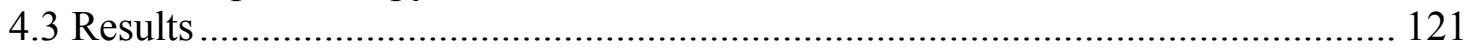

4.3.1 Proton NMR of the Ferric Forms of the Native Low-Spin Camphor-Free P450cam and the Native High-Spin Camphor-Bound P450cam ................ 121

4.3.2 Proton NMR of the Ferric Low-Spin Cyanide-Adducts of Camphor-Free and Camphor-Bound P450cam ……………………........................... 122

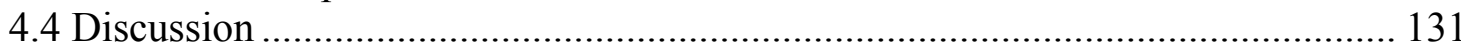

4.4.1 Assignment of the Hyperfine-Shifted Resonances for Ferric Camphor-Free P450cam-CN- Complex …….............................................................. 131

4.4.2 Assignment of the Hyperfine-Shifted Resonances for Ferric Camphor-bound

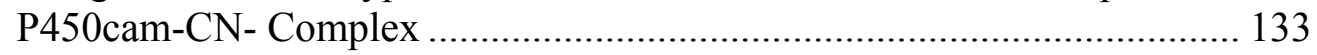

4.4.3 The Heme thiolate ligand Cys357 ......................................................... 135

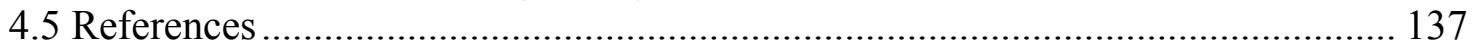

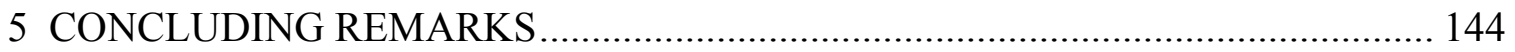

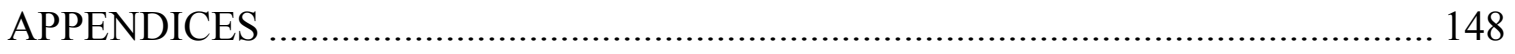

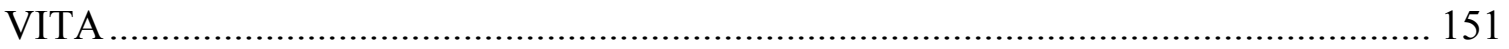




\section{LIST OF FIGURES}

FIGURE

PAGE

1.1 Hemes commonly found in biological systems ............................................... 3

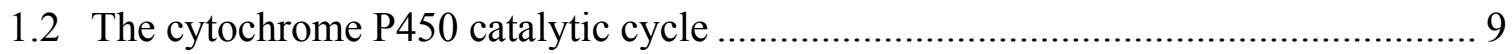

1.3 Schematic structures obtained from the crystallographic results of P450s............. 10

1.4 Schematic structure obtained from the crystallographic result of chloroperoxidase... 14

1.5 The heme active site of chloroperoxidase .................................................. 14

1.6 The chloroperoxidase catalytic cycle ............................................................. 15

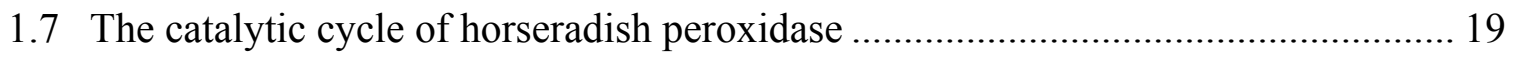

1.8 The highly conserved active site structures of three peroxidases ......................... 20

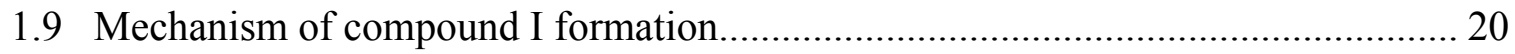

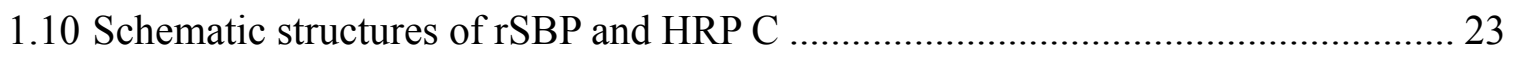

2.1 MS and MS/MS spectra of purified green species extracted from green CPO ........ 51

$2.2 \mathrm{MS} / \mathrm{MS}$ spectra of depolymerized green heme …........................................... 52

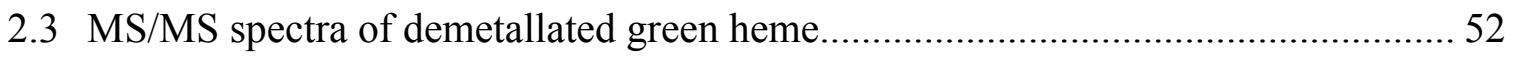

2.4 UV-vis spectra of the green heme dimer, green heme monomer and green heme cyanide derivative in methanol .................................................... 54

2.5 The proton NMR spectra of the green heme dimer, monomer, green heme- $\mathrm{CN}^{-}$adduct and demetallated green heme .............................................. 56

2.6 The 600-MHz phase-sensitive ${ }^{1} \mathrm{H}$ NOESY spectra of $N$-ABPP ............................. 58

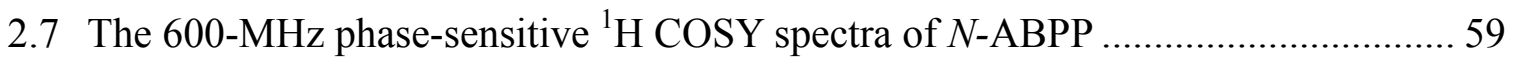

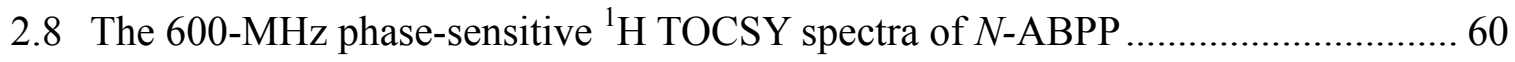

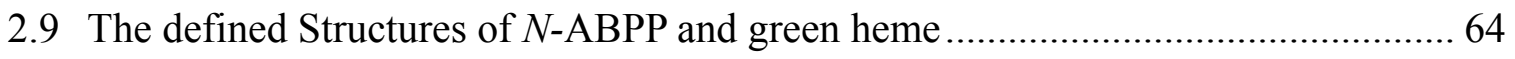

2.10 Hypothesized catalytic pathways of epoxidation and $N$-alkylation........................ 66 
2.11 CPO distal side binding pocket with allylbenzene 66

3.1 The $600 \mathrm{MHz}$ proton NMR spectra of native high-spin SBP 83

3.2 The schematic representation of the active site structure of the cyanideligated form of most heme peroxidases 84

3.3 The proton NMR spectra of the low-spin protein-cyanide complexes 85

3.4 The 600-MHz proton NMR spectrum of the low-spin $\mathrm{SBP}_{-} \mathrm{CN}^{-}$complex and the the NOE difference spectra 88

3.5 The 600-MHz proton NMR spectrum of the low-spin $\mathrm{SBP}_{-} \mathrm{CN}^{-}$complex and the the NOE difference spectra

3.6 The 600-MHz proton NMR spectrum of the ferric low-spin $\mathrm{SBP}_{-} \mathrm{CN}^{-}$complex ..... 90

3.7 The 600-MHz phase-sensitive ${ }^{1} \mathrm{H}$ NOESY spectra of $\mathrm{SBP}_{-\mathrm{CN}}{ }^{-}$at $308 \mathrm{~K} \ldots \ldots \ldots \ldots . . . . . .91$

3.8 The 600-MHz phase-sensitive ${ }^{1} \mathrm{H}$ NOESY spectra of $\mathrm{SBP}_{-\mathrm{CN}^{-}}$at $313 \mathrm{~K} \ldots \ldots \ldots \ldots . . . . . .92$

3.9 The 600-MHz phase-sensitive ${ }^{1} \mathrm{H}$ NOESY spectra of $\mathrm{SBP}_{-\mathrm{CN}}{ }^{-}$at $323 \mathrm{~K} \ldots \ldots \ldots \ldots . . . . . .93$

3.10 The 600-MHz phase-sensitive ${ }^{1} \mathrm{H}$ COSY spectra of SBP-CN ${ }^{-}$at $323 \mathrm{~K} \ldots \ldots \ldots \ldots . . . . . .94$

3.11 Curie plots of the resolved hyperfine shifted resonances of SBP-CN ${ }^{-} \ldots \ldots \ldots \ldots \ldots \ldots . . . . . . .96$

3.12 The 600-MHz proton NMR spectra of calcium "depleted" SBP-CN' ${ }^{-}$.................... 97

4.1 Schematic presentation of the iron protoporphyrin IX in P450cam.......................116

$4.2600 \mathrm{MHz}{ }^{1} \mathrm{H}$ NMR spectra of the ferric forms of P450cam.............................. 123

4.3 The $600 \mathrm{MHz}$ phase-sensitive ${ }^{1} \mathrm{H}$ NOESY spectra of cam-free P450cam-CN ${ }^{-} \ldots . . .127$

4.4 The $600 \mathrm{MHz}$ phase-sensitive ${ }^{1} \mathrm{H}$ NOESY spectra of cam-bound P450cam-CN ${ }^{-}$.. 128

4.5 The 600-MHz proton NMR spectrum of cam-bound $\mathrm{P} 450$ cam- $\mathrm{CN}^{-}$ and the the NOE difference spectra

4.6 The $600 \mathrm{MHz}$ phase-sensitive ${ }^{1} \mathrm{H}$ COSY spectra of cam-bound $\mathrm{P} 450 \mathrm{cam}-\mathrm{CN}^{-} \ldots . .130$

4.7 Curie plots of the hyperfine shifted resonances of cam-bound $\mathrm{P} 450 \mathrm{cam}-\mathrm{CN}^{-} \ldots \ldots .131$ 


\section{LIST OF TABLES}

TABLE

PAGE

1.1 H Reactions catalysed by cytochromes P450 ................................................... 7

2.1 Proton NMR chemical shifts of the resonances for the $N$-ABPP and $N_{\mathrm{III}}-\mathrm{MePP} \ldots \ldots .57$

3.1 Proton NMR chemical shift, $\mathrm{T}_{1}$, and Curie plots intercept values of the resonances for the low-spin cyanide derivatives of SBP, $\mathrm{HRP}$ and $\mathrm{CcP}$

3.2 Proton NMR chemical shift values of the diamagnetic resonances for the low-spin cyanide derivatives of SBP and HRP

3.3 Proton NMR chemical shift values of the isotropically shifted resonances for the high-spin native SBP and HRP

4.1 Proton NMR values of the assigned resonances for the low-spin cyanide derivatives of cam-free and cam-bound P450cam, and CPO....

4.2 Distances between proximal Cys $\beta$-protons and selected atoms in the low-spin cyanide derivatives of cam-free and cam-bound P450cam, and CPO 


\section{LIST OF ABBREVIATIONS}

1D

One dimensional

2D

Two dimensional

$\mathrm{AB}$

Allylbenzene

ABTS

2,2'-azino-bis(3-ethylbenzthiazoline-6-sulfonic acid) diammonium salt

cam

Camphor

$\mathrm{CcO}$

Cytochrome $c$ oxidase

$\mathrm{CcP}$

Cytochrome $c$ peroxidase

CMP

Coprinus macrorhizus peroxidase

$\mathrm{CN}$

Cyanide

CooA

CO-sensing transcriptional activator

COSY

Correlation spectroscopy

Cpd 0

Compound 0

Cpd I

Compound I

$\mathrm{CPO}$

Chloroperoxidase

CW

Q-band continuous wave

CYP

Cytochrome P450

DEAE

Diethylaminoethyl (cellulose)

DMSO

Dimethyl sulfoxide

DTT

Dithiothreitol

EDTA Ethylenediaminetetraacetic acid

ENDOR Pulsed electron nuclear double resonance spectroscopy

EPR Electron paramagnetic resonance 


$\begin{array}{ll}\text { FPS } & \text { Fructose Peptone Salts } \\ \text { HPLC } & \text { High-performance liquid chromatography } \\ \text { HRP } & \text { Horseradish peroxidase } \\ \text { HRP-C } & \text { Horseradish peroxidase C isoenzyme } \\ \text { IPTG } & \text { Isopropyl } \beta \text {-D-1-thiogalactopyranoside } \\ \text { KCN } & \text { Potassium cyanide } \\ \text { MALDI } & \text { Matrix-assisted laser desorption/ionisation } \\ \text { MCD } & \text { Monochlorodimedone } \\ \text { MnP } & \text { Manganese peroxidase } \\ N \text {-ABPP } & N \text {-allylbenzene-protoporphyrin adduct } \\ \text { NADH } & \text { Nicotinamide adenine dinucleotide, reduced } \\ \text { NADPH } & \text { Nicotinamide adenine dinucleotide phosphate, reduced } \\ N \text {-GfPP } & N \text {-griseofulvin-protoporphyrin } \\ N \text {-MePP } & N \text {-methylprotoporphyrin } \\ \text { NMR } & \text { Nuclear magnetic resonance } \\ \text { NOE } & \text { Nuclear Overhauser effect } \\ \text { NOESY } & \text { Nuclear Overhauser enhancement spectroscopy } \\ \text { NOS } & \text { Nitric oxide synthase } \\ \text { P450 } & \text { Cytochrome P450 } \\ \text { PDA } & \text { Potato Dextrose Agar } \\ \text { PMSF } & \text { Phenylmethylsulfonyl fluoride } \\ \text { RBP } & \text { Recombinant SBP } \\ \text { Heitzahl value }\end{array}$




\begin{tabular}{ll} 
SBP & Soybean peroxidase \\
SDS & Sodium dodecyl sulfate \\
TOCSY & Total correlation spectroscopy \\
TOF & Time of flight \\
Tris & Tris(hydroxymethyl)aminomethane \\
WT & Wild type \\
Ala & Alanine \\
Arg & Arginine \\
Asn & Asparagine \\
Cys & Cysteine \\
Glu & Glutamic acid \\
His & Histidine \\
Ile & Isoleucine \\
Leu & Leucine \\
Phe & Phenylalanine \\
Trp & Tryptophan \\
Val & Valine \\
\hline
\end{tabular}




\section{CHAPTER I. LITERATURE REVIEW}

\subsection{Introduction to Hemoproteins}

Hemoproteins (EC 1.11.1.7) are one of the largest classes of metalloproteins that are ubiquitous and essential for every organism and are involved in a wide range of biological processes while retaining a basically unaltered prosthetic group consisting of an iron-protoporphyrin IX (heme). Hemoproteins are therefore good models for exploring the relationship between protein structure and function. However, after over eighty years of research, the relationship between the various porphyrin architectures and the biochemical function of hemoproteins remains poorly defined.

The biological functions of hemoproteins include: electron transfer (b- and c-type cytochromes), oxygen transport (hemoglobin) and storage (myoglobin), oxygen reduction (cytochrome oxidases), organic substrates oxygenation (cytochromes P450), small molecules sensing (CooA), and peroxides reduction (catalases and peroxidases) (1-2). Studies of hemoproteins have suggested that the flexibility in hemoprotein functions normally arises from a combination of differences in both heme constituents and the polypeptide of the various hemoproteins (3).

Concerning the prosthetic heme group, there are four common representatives, that contain a planar iron-tetrapyrrole ring system with different side chains (Figure 1.1). Heme $a$ provides cofactors for the cytochrome $a a_{3}$ of cytochrome $c$ oxidase $(\mathrm{Cc} \mathrm{O})$, an important respiratory protein (4). Heme- $b$ (also termed iron-protoporphyrin IX) is the most abundant and important among these four hemes, since heme $b$ forms the prosthetic group of hemoglobin, myoglobin, catalase, most peroxidases, the b-type cytochromes and the cytochrome P-450s. Heme $c$ is the prosthetic group for the c-type 
cytochromes, which are the most extensively studied family of electron transfer proteins (5). Heme $d_{1}$ is found as a prosthetic group in the heme-containing nitrite reductases designated as cytochromes $c d_{1}(6)$. Since heme $b$ is the only prosthetic group of all the hemoproteins related to my work, I will limit my discussion to enzymes containing a heme $b$.

Heme $b$ has eight side chains which are conventionally numbered as position 1-8 in a clockwise sense as shown in Figure 1.1 B: four methyl groups located at positions 1, 3,5 , and 8, two vinyl groups at positions 2 and 4, and two propionate groups at positions 6 and 7. The carbon atoms of the methine bridges are named separately from the side chains as $\alpha, \beta, \gamma$, and $\delta$, which results in the hydrogen on each methine bridge carbon named as meso- $\alpha, \beta, \gamma$, and $\delta$ proton, respectively. The four pyrrole rings are, clockwise, named as pyrrole ring I, II, III and IV. The iron atom is placed in the center of the heme with the four pyrrole nitrogens equatorially ligated. In hemoproteins, the heme iron normally has two axial ligands binding sites which are located above (termed distal side) and below the porphyrin plane (termed proximal side). The importance and function of these two axial ligands will be discussed later.

Heme $b$ in hemoproteins can exist as two rotational isomers called isomers A and B that differ by a $180^{\circ}$ rotation of the heme group about the $\alpha-\gamma$ axis, and leads to different protein-heme contacts. For isomer A, the porphyrin substituents are arranged in a clockwise sense when viewed from the proximal side of proteins, such as chloroperoxidase (CPO) and horseradish peroxidase (HRP). For isomer B, the porphyrin substituents are arranged in a counterclockwise sense, such as cytochrome $c$ peroxidase $(\mathrm{C} c \mathrm{P})$ and manganese peroxidase $(\mathrm{MnP})$. Two-fold rotation about the 


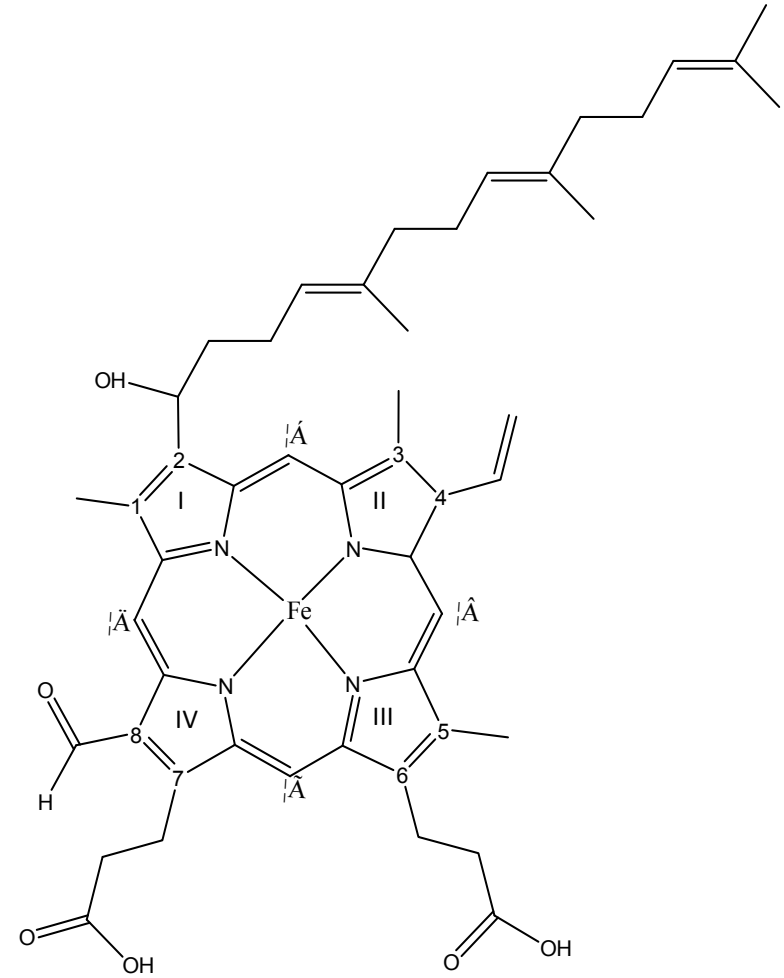

A. Heme $a$

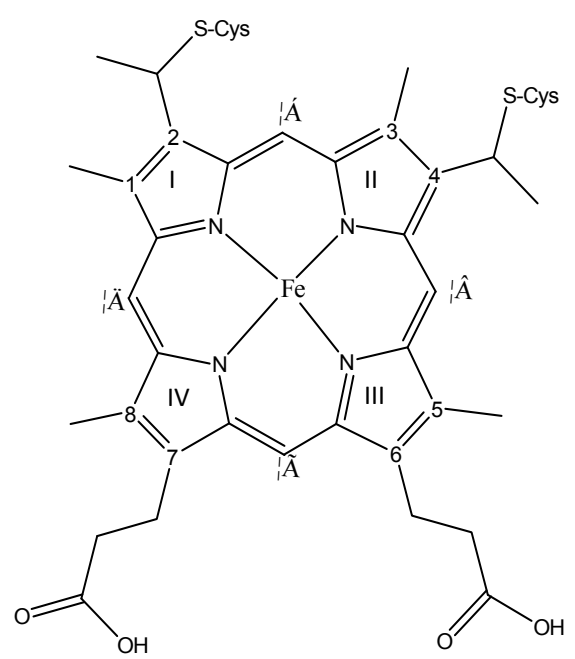

C. Heme $c$

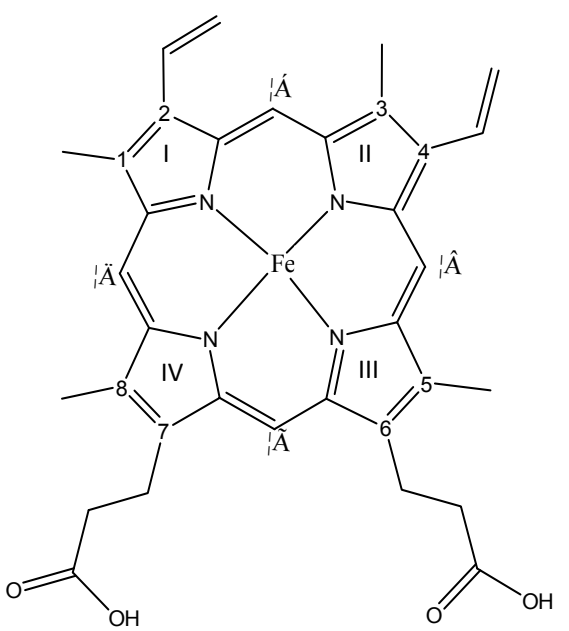

B. Heme $b$

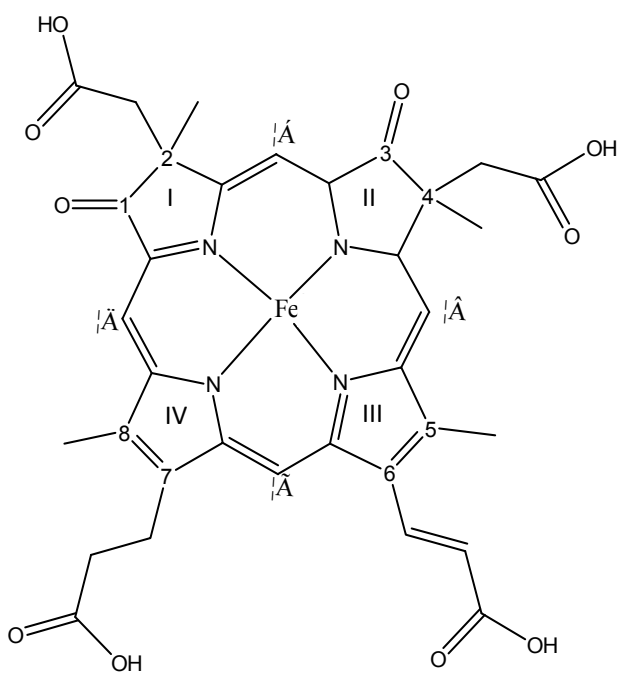

D. Heme $d_{1}$

Figure 1.1 Hemes commonly found in biological systems. 
$\alpha-\gamma$ axis interchanges the 2- and 4-vinyl groups with the 3- and 1-methyl groups, respectively. Studies by NMR have shown that the equilibrium ratio of A to B form depends on the protein sequence, and the oxidation state and the ligands of the heme (7).

Concerning the polypeptide part of the molecule, it is obvious that the protein structure around the heme center is of great importance in controlling the type of reaction catalyzed, and directing the heme to a prescribed function specifically and efficiently. The environment surrounding the heme iron in hemoproteins dictates their activity and makes them a diverse group of enzymes. In particular, axial coordination to the central iron atom, covalent attachment of the heme to the protein, H-bonding interactions, conformations, and amino acid side chains in the immediate vicinity of the active site, are all of dominant importance in governing the redox potentials and enzymatic functions.

The presence and the nature of the fifth and sixth axial ligands to the iron will be crucial in determining the reactivity of hemoproteins. For example, in oxidases or oxygenases which need to bind oxygen or hydrogen peroxide, it is obviously of paramount importance to have a coordination site (distal site) available for exogenous ligands. At the same time, the proximal site will be occupied by heteroatoms from nucleophilic amino acid residues. The distal position in these hemoproteins is normally the catalytic center and its coordination state varies during the catalytic cycle. Meanwhile the proximal ligand is highly conserved and is used as an important indication to differentiate each hemoprotein families. For example, in all heme peroxidases except chloroperoxidase, the proximal ligand is always a histidine nitrogen; while in catalase, it is a tyrosine oxygen, and in all $\mathrm{P} 450 \mathrm{~s}$ and $\mathrm{CPO}$, it is a cysteinate sulfur serving as the proximal ligand. In contrast, the hemoproteins involved in simple electron transfers, 
which do not directly bind exogenous ligands but need to avoid reorganization at the heme iron site, are therefore almost invariably hexa-coordinated with the iron in the lowspin state with both axial sites occupied by heteroatoms from the peptide backbone amino acids (8). Because of the variety and complexity of hemoproteins, I will only further introduce several hemoproteins which are closely related to my researches in this chapter.

\subsection{Heme-Thiolate Proteins}

As mentioned above, the proximal heme ligand of hemoprotein is an important indication for classification. Therefore, hemoproteins using a thiolate anion from a deprotonated cysteine residue as the proximal ligand are grouped as heme-thiolate proteins, in contrast with another major hemoprotein category, the heme-imidazole proteins, whose proximal ligand is from a histidine imidazole nitrogen.

The known heme-thiolate proteins currently include all cytochromes P450, CPO, nitric oxide synthases (NOS), cystathionine $\beta$-synthase, sensor protein CooA and eIF2 $\alpha$ kinase. With the same proximal ligand, heme-thiolate proteins exhibit highly versatile, diverse and essential functions in biological systems. The remainder of this chapter will focus on the structures, functions, and mechanisms of cytochromes P450 and CPO that constitute the major part of my dissertation research.

\subsubsection{Cytochromes P450}

Cytochromes P450 (CYP) are a family of monooxygenases (mixed function oxidases) named for their characteristic absorption maximum (the Soret peak) at around $450 \mathrm{~nm}$ upon binding of carbon monoxide by the reduced protein. The title of "cytochrome" is earned because of the electron transfer functionality and colorful character of the P450 enzymes. Cytochrome P450 monooxygenases require only two electrons and two protons 
to reductively cleave atmospheric dioxygen, producing a single water molecule in the process while saving the second oxygen atom for substrate functionalization and formal oxidation (Equation 1). This two-electron reduction process is in contrast with dioxygenases which need the full four-electron reduction of dioxygen to generate two water molecules (9). Electrons that are required for the catalysis were found to come from NADH or NADPH via electron transfer proteins, such as cytochrome P450 ferredoxin and cytochrome P450 reductase.

$$
\mathrm{R}-\mathrm{H}+\mathrm{O}_{2}+2 \mathrm{e}^{-}+2 \mathrm{H}^{+} \longrightarrow \mathrm{ROH}+\mathrm{H}_{2} \mathrm{O}
$$

Ever since the first P450 was discovered in 1958 (10-11), massive amount of effort has been put into studying the ubiquitous P450s for over half a century. Many reviews ( 9 , 12-22) and books (23-28) have been devoted to the superfamily of P450s as catalyst. There are two main features that attracted the interest of chemists: (i) the P450s can catalyze the selective monooxygenation of a wide variety of organic substrate, and (ii) the P450s play a key role in the oxidative metabolism of drugs and other xenobiotics including steroid hormones, which is a crucial step in the adaptation of living organisms to their always changing chemical environment (18). The intense research performed on P450s has revealed more than 11,500 distinct proteins that are spread across almost all living organisms (9). All P450s have the highly conserved cysteine thiolate acting as the heme proximal ligand, which is considered to play critical roles in the catalytic mechanisms. Although the sequence homology between P450 members from different gene sources can be as low as $\sim 20 \%$ on the amino acid level, their overall structures have high similarity. 
The P450s have revealed a high diversity of biological functions, catalyzed reactions, and acceptable substrates $(19,21-22)$. The biological functions of P450s include the biosynthesis and biodegradation of important endogenous compounds and the metabolism of xenobiotics as mentioned above. The reactions catalyzed by P450s are listed in Table 1.1. The substrates for P450s include fatty acids, steroids, prostaglandins, as well as a multitude of foreign compounds such as drugs, anaesthetics, organic solvents, ethanol, alkylaryl hydrocarbon products, pesticides, and carcinogens.

Table 1.1 Reactions catalyzed by cytochromes P450 (14, 19, 22, 29).

\begin{tabular}{ll}
\hline Hydrocarbon hydroxylation & Alkene epoxidation \\
Alkyne oxygenation & Arene epoxidation \\
Aromatic hydroxylation & N- Dealkylation \\
S- Dealkylation & O- Dealkylation \\
N- Hydroxylation & N- Oxidation \\
S- Oxidation & Oxidative deamination \\
Dehydratations & Alcohol and aldehydes oxidations \\
Oxidative dehalogenation & Reductive dehalogenation \\
N- Oxide reduction & Epoxide reduction \\
Reductive $\beta$-scission of alkyl peroxides & NO reduction \\
Isomerizations & Oxidative C-C bond cleavage \\
Allylic rearrangement & Sulfoxidation \\
Desaturation & Deformylation \\
Peroxidase-type oxidation & NO-Synthase-type oxidation \\
\hline
\end{tabular}


The detailed catalytic cycle of dioxygen activation and substrate hydroxylation derived from the most studied P450, the camphor monooxygenase, P450cam, from Pseudomonas putida, is shown in Figure 1.2. The resting state enzyme [1] is in an oxidized ferric low-spin, six-coordinate form with a water molecule serving as the distal ligand. Upon substrate binding, the distal water is removed, leaving the heme iron as a high-spin, five-coordinate form shown as intermediate [2] (19), and meanwhile the reduction potential is increased to allow electron transfer to occur. The water exclusion process initiated the first electron transfer to the heme iron from $\mathrm{NAD}(\mathrm{P}) \mathrm{H}$ via electron transfer proteins, yielding a reduced P450 with a five-coordinate heme (intermediate [3]). The binding of dioxygen forms an unstable ferrous-oxy intermediate [4]. The following second electron transfer step, which is the rate limiting step, forms a ferric peroxo-iron species [5], which is consequently protonated to the formation of the hydroperoxo-iron complex [6], Compound $0(\mathrm{Cpd} 0)$. Another proton is transfered into the active site to the distal oxygen to initiate the formation of a highly reactive $\mathrm{Fe}^{\mathrm{IV}}=\mathrm{O}$ (ferryl-oxo) complex [7], Compound I (30), which is the most widely accepted key intermediate in P450catalyzed reactions. The Compound I will hydroxylate the bound substrate and finish the reaction cycle by releasing the product and adopting a water molecule to regenerate the resting state enzyme.

As previously mentioned, $\mathrm{P} 450$ s from different sources are found to preserve a highly similar secondary and tertiary structure in spite of their low amino acid sequence homology $(9,17)$. Crystal structures of four P450s: P450 cam, P450 $0_{\mathrm{BM}-3}, \mathrm{P} 450_{\text {eryF }}$ and P450 terp, which are shown in Figure 1.3, exhibit very similar topology. The two 


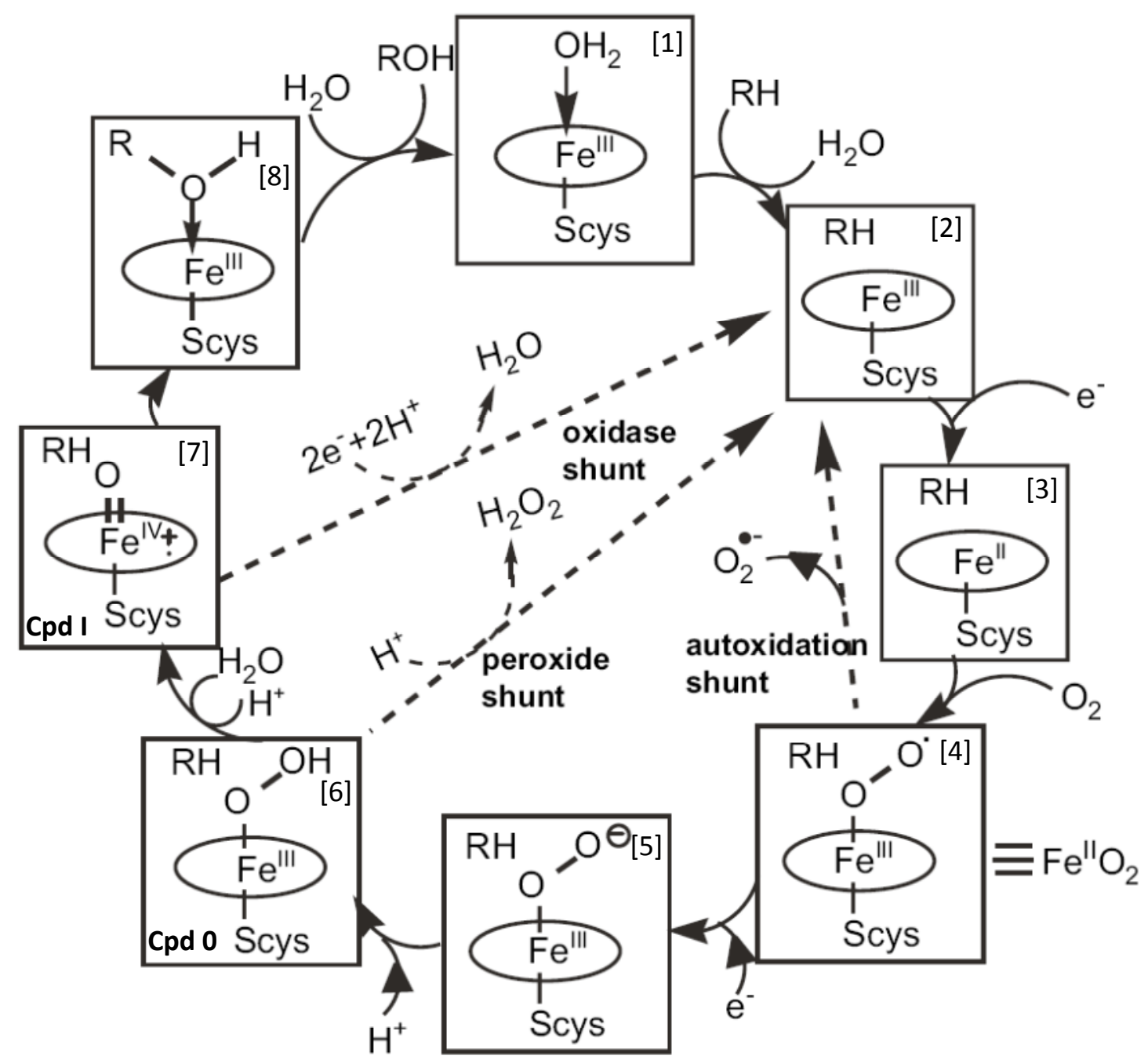

Figure 1.2 The cytochrome P450 catalytic cycle (23). RH is substrate and ROH is product; Scys is the cysteinate ligand; the heme porphyrin is represented as a circle. Decoupling pathways resulting from oxidase activities of P450, in competition with the monooxygenase activity, are called autoxidation, and peroxide and oxidase shunts.

hydrophobic helices which sandwich the heme prosthetic group from the proximal and distal direction, respectively, are similarly positioned in all P450s, leaving no part of the heme directly exposed to bulk solvent. The highly conserved cysteine ligand is situated at the N-terminal end of the proximal helix.

Cytochrome P450 cam (CYP101) is the first to be completely purified (31) and analyzed by X-ray diffraction (32-33) and the most studied among all P450 members. 

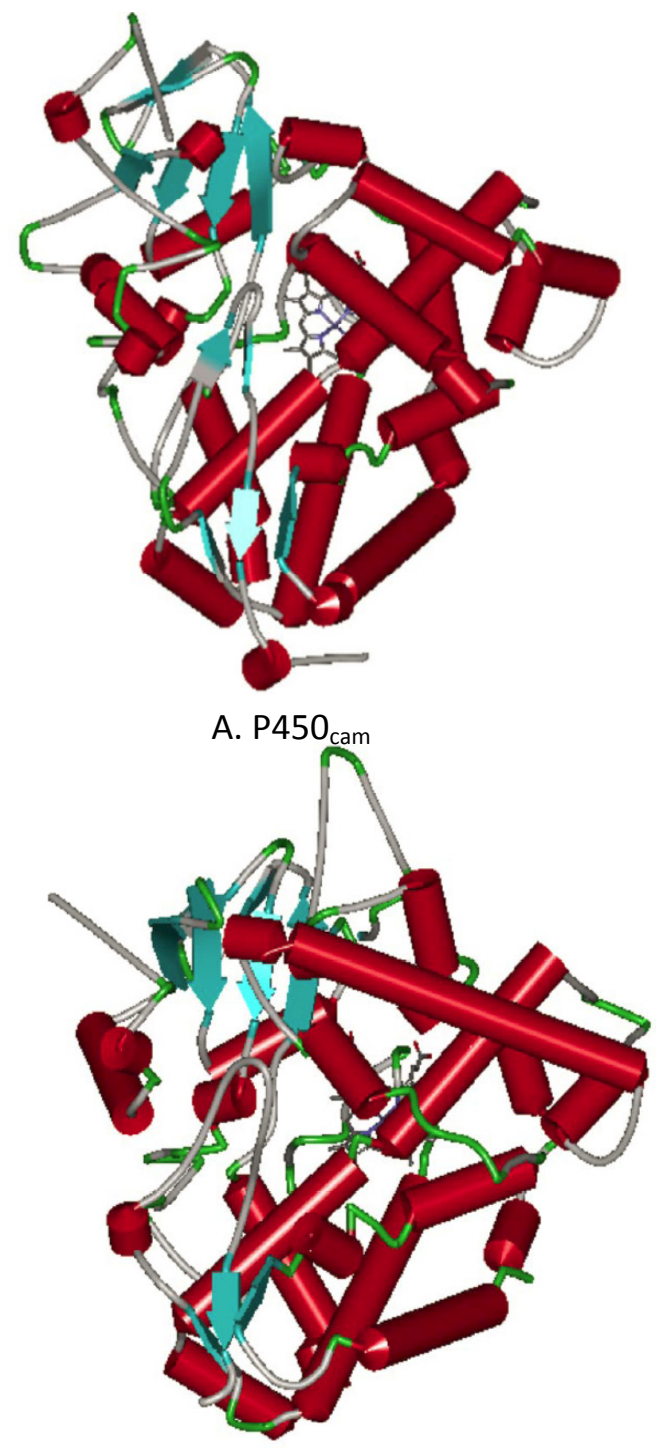

C. $P 450_{\text {eryF }}$

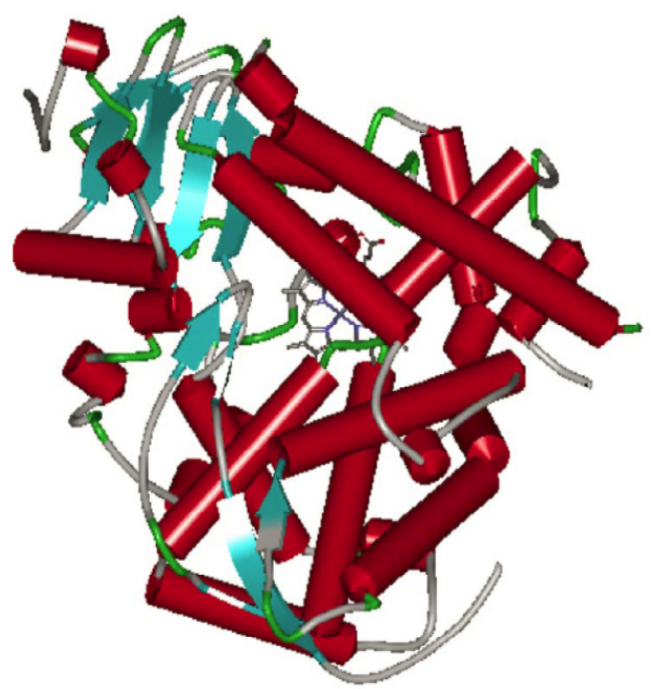

B. $P 450_{\mathrm{BM}-3}$

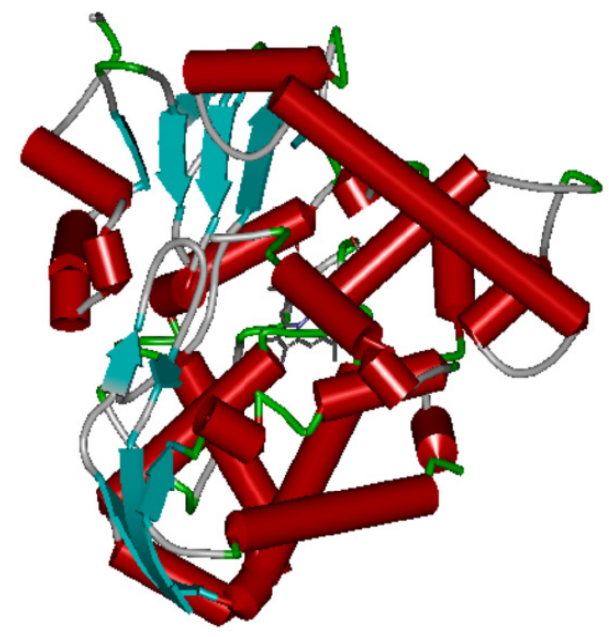

D. $P 450_{\text {terp }}$

Figure 1.3 Schematic structures obtained from the crystallographic results of: A. P450 $0_{\text {cam }}$ (PDB code: 2CPP), B. P450 $0_{\mathrm{BM}-3}$ (PDB code: 1JPZ), C. P450 eryF (PDB code: 1EUP) and P450 $0_{\text {terp }}$ (PDB code: 1CPT). The helix-rich domain is shown on the right side and the helix-poor domain on the left side.

A large amount of crystal structure studies of substrate/inhibitor complexes of P450 $0_{\text {cam }}$ provided important insights into the relationship between substrate binding, spin state, redox potential and stereospecificity. However, although $\mathrm{P} 450_{\text {cam }}$ is the best characterized among the P450 superfamily, a fundamental question remains to be addressed: How does 
the substrate fit into the active site of the enzyme? This mystery has troubled researchers for a long time because all crystal structures of $\mathrm{P} 450_{\text {cam }}$, no matter substrate-free or substrate-bound form, are always in a static conformation with no obvious path into the active site and provided only limited information regarding the dynamics of the binding process. The "invisible" structural and conformational changing process upon substrate binding is partly revealed by my NMR research of P450cam and is described in detail in Chapter 4.

\subsubsection{Chloroperoxidase}

Chloroperoxidase (CPO) was first discovered by Hager and coworkers as a chlorinating enzyme from a marine fungus, Caldariomyces fumago (34). During the last half century of study, CPO has been found to be one of the most diverse and versatile catalysts among the hemoprotein superfamily. In addition to its biological role of halogenating organic molecules (Equation 2) (35), CPO was also found to possess peroxidase (Equation 3) and catalase (Equation 4) activities (36), together with P450-like oxygen insertion reactions (Equation 5) without any requirement for NADH or coenzyme (37). In general, CPO can utilize hydrogen peroxide or organic hydroperoxide as the oxidant to catalyze a wide variety of one- and two-electron oxidations, such as the oxidation of alcohols to aldehydes, aldehydes to acid, and amines to nitroso compounds, propargylic oxidation (38-40), $\mathrm{N}$-demethylation and dealkylation (41), dimerization of phenols, stereospecific hydroxylation and chiral sulfoxidations (39, 42, 43). Chloroperoxidase can also catalyze enantioselective epoxidation of cis-disubstituted alkenes with high turnovers in concert with high enantiomeric excess (ee) for a range of aliphatic and aromatic olefins in this class $(38,44-45)$. This capability makes CPO a 
promising candidate to be an important industrial catalyst because chiral epoxides are very useful to form bifunctional compounds via stereo specific ring-opening.

$$
\begin{array}{ll}
\text { Halogenation (normal function): } & \mathrm{RH}+\mathrm{H}_{2} \mathrm{O}_{2}+\mathrm{Cl}^{-}+\mathrm{H}^{+} \longrightarrow \mathrm{R}-\mathrm{Cl}+2 \mathrm{H}_{2} \mathrm{O} \\
\text { Dehydrogenation (peroxidases): } & 2 \mathrm{RH}+\mathrm{H}_{2} \mathrm{O}_{2} \longrightarrow \mathrm{R}-\mathrm{R}+2 \mathrm{H}_{2} \mathrm{O} \\
\text { Peroxide decomposition (catalase): } & 2 \mathrm{H}_{2} \mathrm{O}_{2} \longrightarrow \mathrm{O}_{2}+2 \mathrm{H}_{2} \mathrm{O} \\
\text { Oxygen insertion (P450): } & \mathrm{RH}+\mathrm{H}_{2} \mathrm{O}_{2} \longrightarrow \mathrm{R}-\mathrm{OH}+\mathrm{H}_{2} \mathrm{O}
\end{array}
$$

In addition to catalyzing a wide range of reactions, $\mathrm{CPO}$ also serves as a unique and precious structure model for the study of the mechanism of hemoproteins. Chloroperoxidase, composed of 299 amino acid residues, is a typical heme enzyme containing ferric protoporphyrin IX as its prosthetic group. Although CPO shares a number of physiochemical functions with cytochromes P450, the distal pocket of CPO possesses a hydrophilic environment similar to those of heme peroxidases and catalases (46-47). However, in contrast to other peroxidases, the proximal side of the heme active site in CPO has the same feature as cytochromes P450 with a thiolate cysteine as the proximal heme ligand rather than a histidine as in other peroxidases (48-49). The electron-rich thiolate ligand has been generally believed to be critical to the cleavage of the peroxide oxygen-oxygen bond in the absence of the assistance provided by polar active site residues as in horseradish peroxidase. Combined with a P450-like proximal heme environment and a peroxidase-like distal environment, $\mathrm{CPO}$ can be considered as a P450-peroxidase hybrid. The hybrid architecture may explain why CPO can catalyze the transformation of such a broad range of substrates and exhibits both P450-type and peroxidase-type activities. 
Although shares functional and structural similarities with other hemoproteins, CPO holds some distinctive features revealed by its crystal structure shown in Figure 1.4 (50). The overall tertiary structure of CPO is dominated by eight helical segments, which bears little resemblance to peroxidases or P450s, implying a separate evolutionary origin. Although the distal pocket environment of $\mathrm{CPO}$ is polar as in other peroxidases, a glutamic acid rather than a histidine is positioned directly adjacent to the peroxide-binding site (Figure 1.5). It has been proposed that this glutamic acid serves as the acid-base catalyst to help cleaving the peroxide bond in the formation of Compound I (Cpd I), an oxyferryl porphyrin $\pi$-cation radical, in CPO (51). The peroxide binding site is flanked by two phenylalanine residues, Phe103 and 186, whose benzene rings are almost parallel with the heme plane. These phenylalanine residues are suggested to play a key role in the binding of hydrophobic substrates. The substrate-binding pocket of CPO more closely resembles P450s in that a small opening above the heme allows access to the ferryl-oxo center in Cpd I. The crystal structure of $\mathrm{CPO}$ also reveals that, as the result of post-translational modification, CPO is heavily glycosylated and the carbohydrate account for about $19 \%$ of the total molecular weight (52).

All reactions catalyzed by $\mathrm{CPO}$ are ping-pong reactions in which Compound I is formed in the initial reaction and subsequently reacts with a second substrate to return the protein to the native state (53). Accidentally, it was discovered that when incubated with allylbenzene $(\mathrm{AB})$ or other selected terminal unsaturated substrates in the presence of hydrogen peroxide, $\mathrm{CPO}$ will be rapidly and quantitatively converted to a green species with a complete vanishing of enzymatic activity (Figure 1.6) (54). This phenomenon 


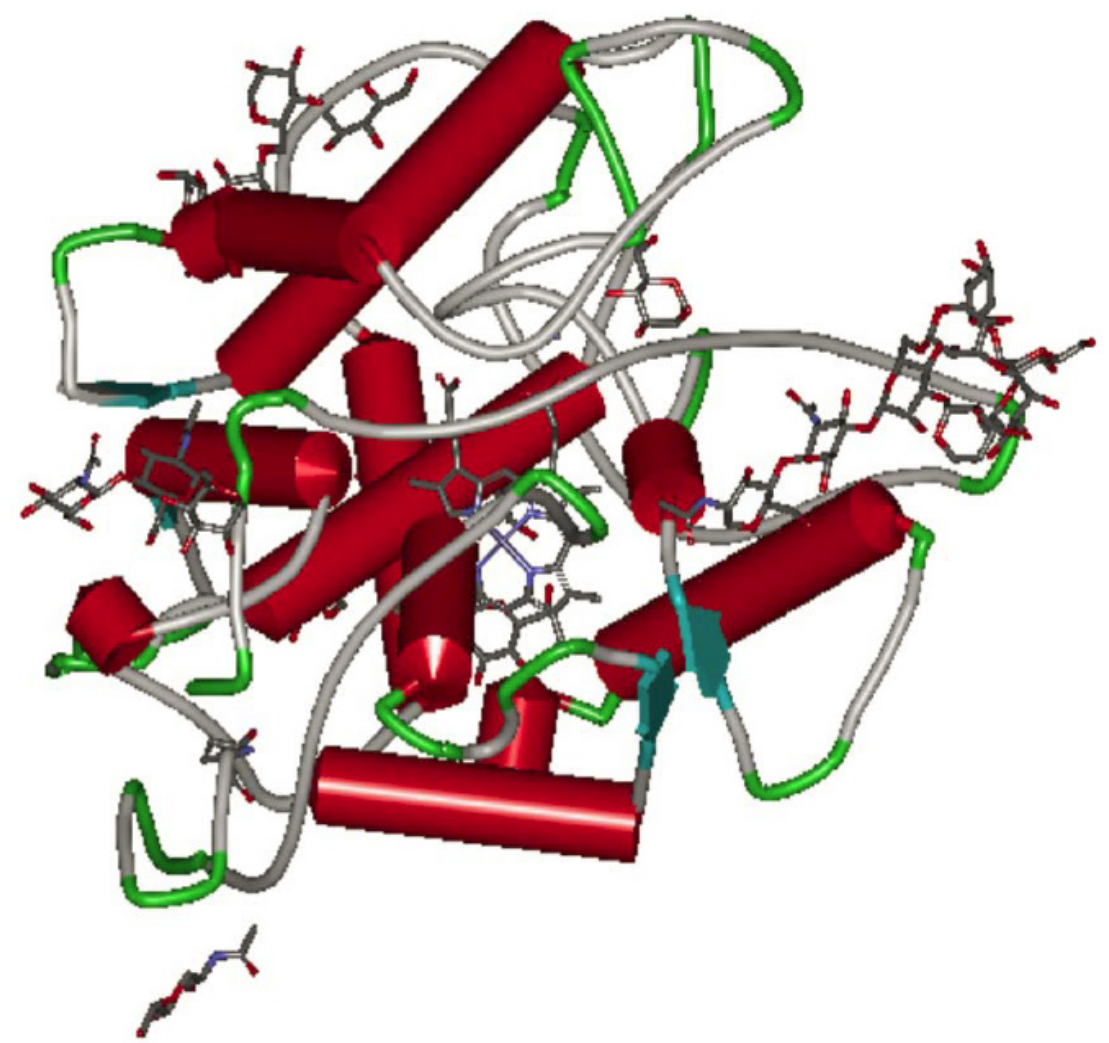

Figure 1.4 Schematic structure obtained from the crystallographic result of chloroperoxidase (PDB code: $2 \mathrm{~J} 5 \mathrm{M})$.
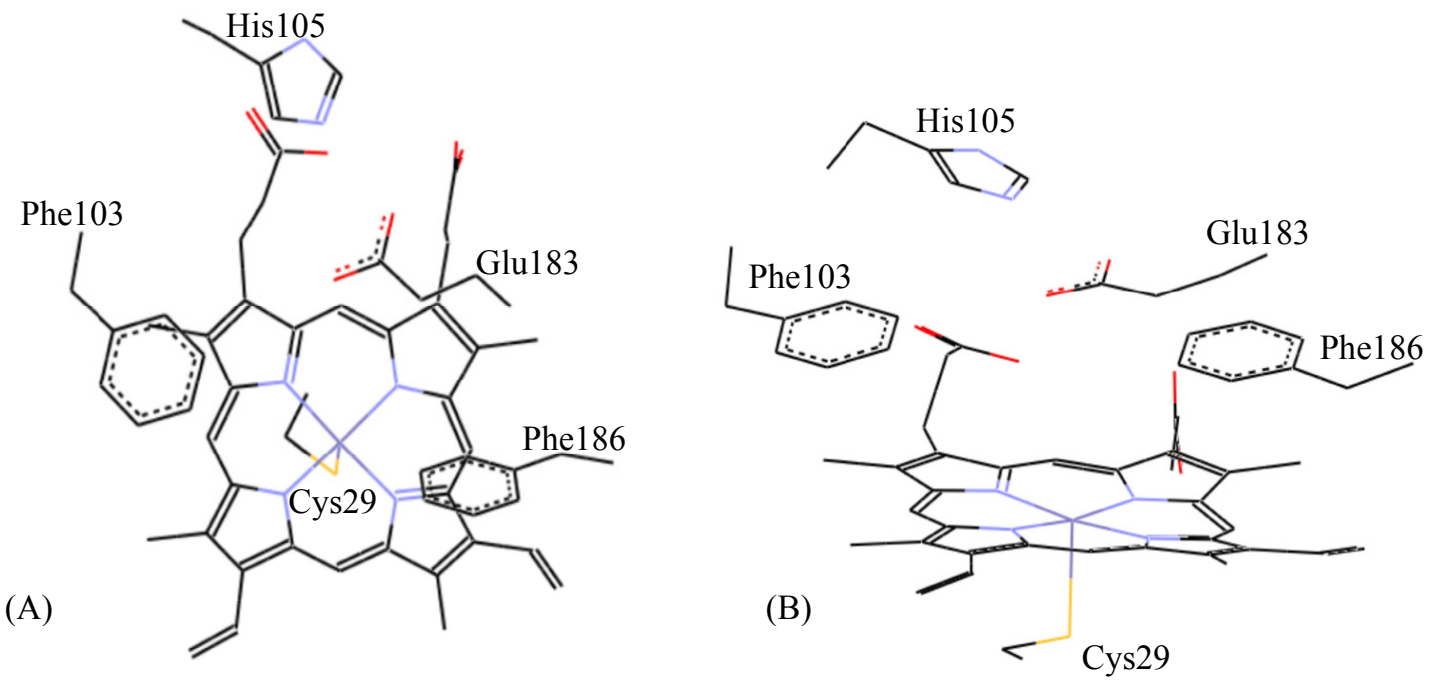

Figure 1.5 The top view (A) and side view (B) of the heme active site of chloroperoxidase. The Glu183 and His105 can form hydrogen bond and function as acid-base catalyst. The two phenylalanine residues may play a role in the binding of hydrophobic residues. 
suggested that $\mathrm{CPO}$ was inactivated in a $\mathrm{P} 450$-type reaction involving the mechanismbased formation of $N$-alkylporphyrins during the oxidation of allylbenzene. Most amazingly, the inactive $\mathrm{CPO}$ (green $\mathrm{CPO}$ ) can slowly return to native $\mathrm{CPO}$ upon standing with a concomitant regain of greater than $80 \%$ of that of the original enzyme's activity (55).

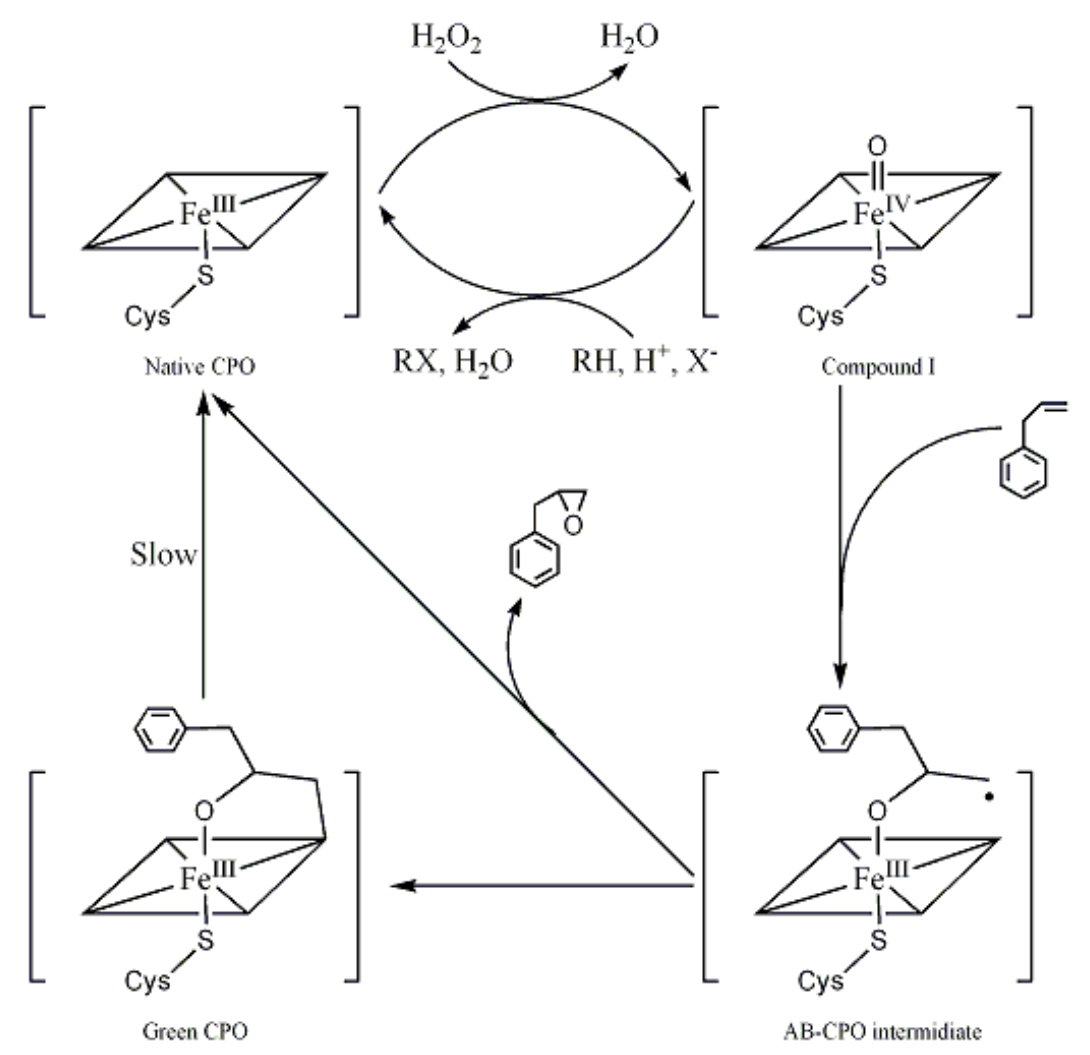

Figure 1.6 The chloroperoxidase catalytic cycle including the allylbenzene inactivation and autorestoration. $\mathrm{RH}$ is substrate and $\mathrm{X}^{-}$is $\mathrm{Cl}^{-}, \mathrm{Br}^{-}$, or $\mathrm{I}^{-}$.

The suicide inactivation of $\mathrm{CPO}$ is shown to be another common reactivity shared with cytochromes $\mathrm{P} 450$ in addition to the structural and functional similarity. The mechanism-based inactivation of P450s also results in "green pigments" with the active site heme being modified by addition of the unsaturated substrate skeleton plus an oxygen atom (56-57). It was reported that the olefin can predominately alkylate the 
nitrogen of pyrrole ring IV of the prosthetic heme, but the acetylenes prefer to alkylate pyrrole ring I (58). The reversible N-alkylation of the prosthetic heme was also reported for a P450 2E1 mutant T303A (59-60).

Q-band continuous wave (CW), pulsed electron nuclear double resonance spectroscopy (ENDOR), Mössbauer and electron paramagnetic resonance (EPR) studies of allylbenzene-inactivated $\mathrm{CPO}$ identified that the green heme species resulted from the $N$-alkylation of the heme with a five-membered chelate ring formed $(55,61)$. However, the techniques used in previous studies were not able to identify which of the four pyrrole ring nitrogen was alkylated. Therefore, a study using NMR was carried out to characterize the modified heme of CPO and the results are described in Chapter II of this dissertation.

\subsection{Heme Peroxidases}

Heme peroxidases (donor: $\mathrm{H}_{2} \mathrm{O}_{2}$, oxidoreductase: EC 1.11.1.7) are a large ubiquitous superfamily of $b$-type hemoproteins that catalyze the oxidation of a number of substrates in the presence of hydrogen peroxide. The reactions catalyzed by heme peroxidases are important to biological systems, ranging from oxidation of aromatic and heteroatom compounds, through free radical oligomerizations and polymerizations of electron-rich aromatics, to electron transfer, sulfoxidation, epoxidation, demethylation and oxidative degradation of lignin (62-63). Nonmammalian heme peroxidases are often grouped with P450s because of having similar intermediates during their catalytic cycles. Nonmammalian heme peroxidases can be divided into three distinct classes (64). Class I are the intercellular peroxidases, such as yeast cytochrome $c$ peroxidase, gene-duplicated bacterial peroxidases and ascorbate peroxidases. Class II are the extracellular fungal peroxidases and class III are the well-known extracellular plant peroxidases. Although 
the primary sequence homology may greatly vary, all none-mammalian heme peroxidases share a common helical-hold overall structure. As mentioned before, all heme peroxidases utilize histidine as the proximal ligand of the heme iron except for chloroperoxidase. In this chapter, I will briefly introduce three important heme peroxidases related to my research: Horseradish peroxidase (HRP), cytochrome $c$ peroxidase $(\mathrm{CcP})$ and soybean peroxidase $(\mathrm{SBP})$.

\subsubsection{Horseradish Peroxidase}

Horseradish peroxidase is one of the most important enzymes obtained from the plant horseradish, Armoracia rusticana, and has been extensively studied for more than a century. Most of our current knowledge about heme peroxidases comes from the studies of HRP. Horseradish peroxidases can utilize hydrogen peroxide to oxidize a wide variety of organic and inorganic compounds. Horseradish peroxidase has been exploited extensively in applications and commercial uses spanning from bioscience to biotechnology, including organic synthesis (65), bioremediation (66), biocatalysis (67), diagnostics (68), biosensors (69), transgenics (70), protein engineering (71) and even to therapeutics (72). Comparing with other popular alternatives such as alkaline phosphatase, horseradish peroxidase has advantages for industrial applications, such as enzyme size, stability, and price aspects. It also has a high turnover rate that allows the generation of strong signals in a relatively short time span. Although the term "horseradish peroxidase" is used generically, the root of the horseradish plant actually contains a number of distinctive peroxidase isoenzymes, among which the $\mathrm{C}$ isoenzyme (HRP-C) is the most abundant and consequently most widely used in studies and applications. 
The HRP-C is a $44 \mathrm{kD}$ glycoprotein containing a single polypeptide of 308 amino acid residues, with two calcium ion binding sites and four disulfide bonds between cysteine residues 11-91, 44-49, 97-301 and 177-209. The two calcium binding sites are located proximal and distal to the heme plane, respectively, and are linked by a network of hydrogen bond to the heme-binding region. The presence of the calcium ions is significant for the structural and functional integrity of HRP-C. Removal of calcium ions results in a decrease in both enzymatic activity and thermal stability (73), and causes subtle changes in the heme environment (74). The calcium ions are also essential for correct folding of denatured or recombinant HRP (75).

Most reactions catalyzed by HRP can be expressed by Equation 6, in which one molecule of hydrogen peroxide is reduced to water and two molecules of radical product, $\mathrm{RH} \bullet$, are generated (70). The catalytic mechanism of HRP-C, has been investigated extensively (Figure 1.7) (65), which involves the formation of the well established and powerful oxidant intermediates: compounds I and II.

$$
2 \mathrm{RH}_{2}+\mathrm{H}_{2} \mathrm{O}_{2} \longrightarrow 2 \mathrm{RH} \bullet+2 \mathrm{H}_{2} \mathrm{O}
$$

Similar as in the peroxide shunt pathway of P450s, the first step in the catalytic cycle of HRP is the reaction that $\mathrm{H}_{2} \mathrm{O}_{2}$ bind to the resting state of the enzymatic heme $\mathrm{Fe}^{\mathrm{III}}$ as the distal ligand. The bound $\mathrm{H}_{2} \mathrm{O}_{2}$ is heterolytically cleaved to generate the high oxidation state intermediate compound $\mathrm{I}$, an $\mathrm{Fe}^{\mathrm{IV}}$ ferryl-oxo center and a porphyrin-based cation radical. Compound I stores two oxidation equivalents, which are used subsequently for substrate oxidation, thereby leading to crucial functions. A putative transient $\mathrm{Fe}^{\mathrm{III}}$ hydroperoxy intermediate (compound 0) formed prior to compound I has been detected in reactions between HRP-C and $\mathrm{H}_{2} \mathrm{O}_{2}$ at low temperatures (76). The highly conserved 
distal histidine and arginine residues (Figure 1.8A) are suggested to cooperatively play important roles through acid-base catalysis in the cleavage of the O-O bond (Figure 1.9) (77-78). The second step in the catalytic cycle is conducted by the first one-electron reduction, which requires the participation of a reducing substrate and leads to the generation of compound II, an $\mathrm{Fe}^{\mathrm{IV}}$ ferryl-oxo species in which the $\mathrm{Fe}^{\mathrm{IV}}=\mathrm{O}$ species remains intact but the porphyrin radical has been reduced (79-80). The catalytic activity of HRP-Compound II is suggested to link to the protonated distal His42 (81). The remaining oxidation equivalent stored in compound II is released consequently in the second one-electron reduction step, which returns the enzyme to its resting state.

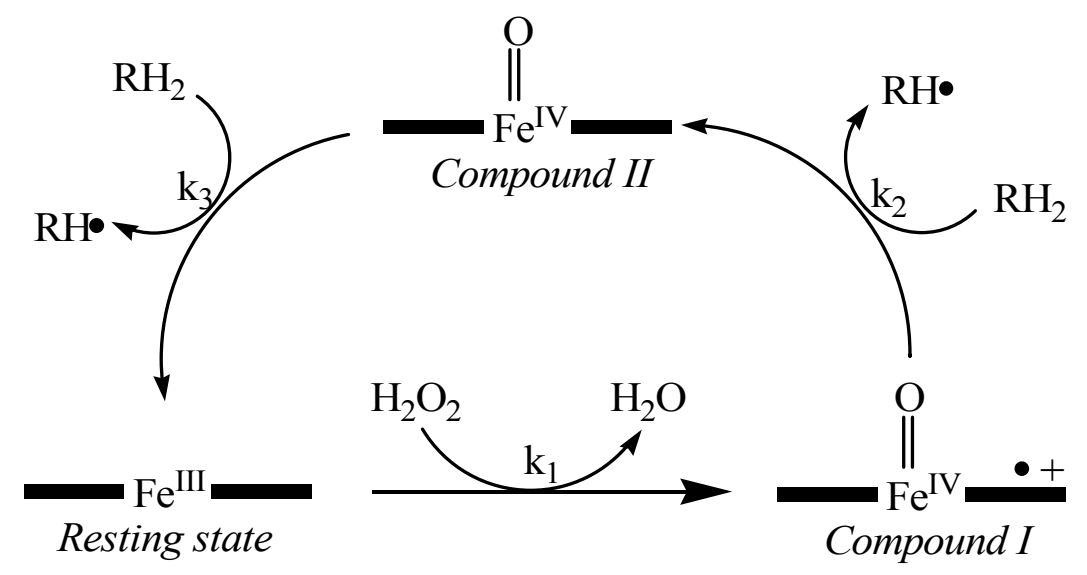

Figure 1.7 The catalytic cycle of horseradish peroxidase (HRP-C). The rate constants $\mathrm{k}_{1}, \mathrm{k}_{2}$ and $\mathrm{k}_{3}$ represent the rate of compound I formation, the rate of compound I reduction and the rate of compound II reduction, respectively. $\mathrm{RH}_{2}$ and $\mathrm{RH} \bullet$ represent a reducing substrate and its radical product, respectively.

Because of the presence of attached carbohydrates which hinders crystal growth, the first crystal structure of HRP-C using X-ray crystallography appeared in the literature relatively late (82) as a recombinant enzyme expressed in Escherichia coli in nonglycosylated form. The structures of HRP-C contain three $\alpha$-helices that are 


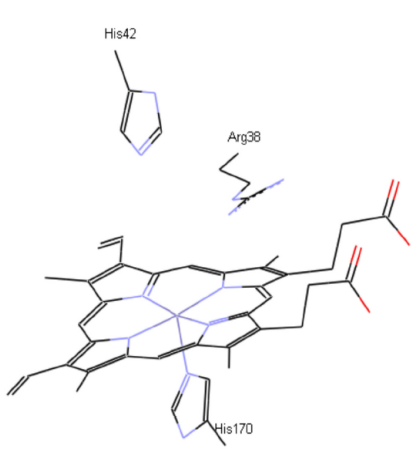

A. HRP-C

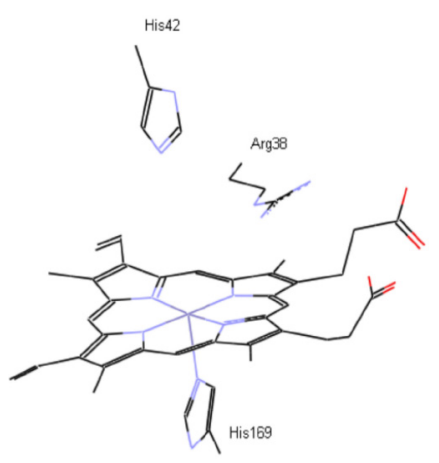

B. SBP

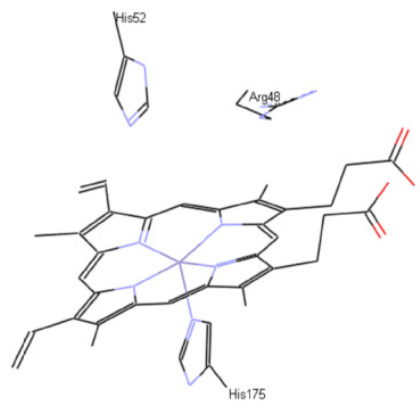

C. $\mathrm{C} c \mathrm{P}$

Figure 1.8 The highly conserved active site structures of (A) horseradish peroxidase C, (B) soybean peroxidase, and (C) cytochrome $c$ peroxidase. The heme group and the catalytic residues Arg, distal His and proximal His are in atom colors.

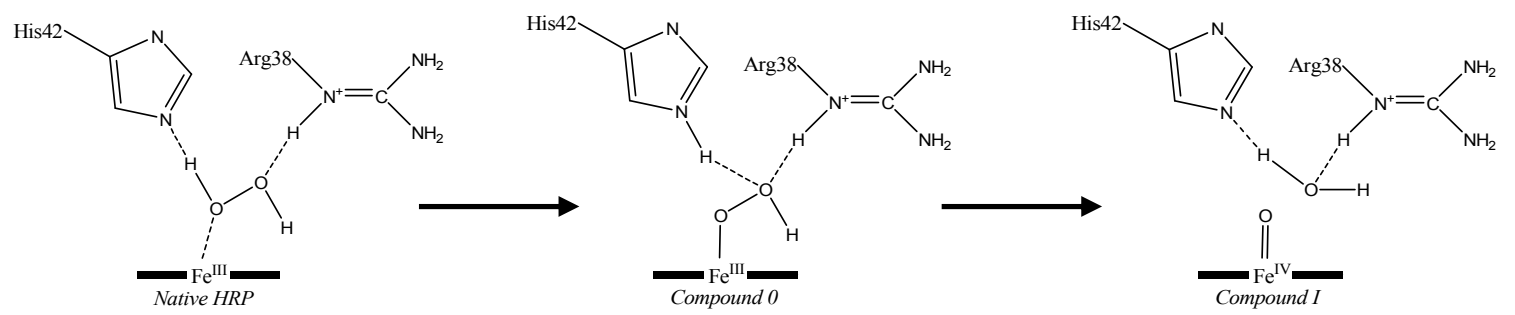

Figure 1.9 Mechanism of compound I formation with the distal His42 plays the main role as both proton acceptor from one of the peroxide oxygen and proton donor to the other peroxide oxygen. The distal Arg38 facilitate the heterolytic cleavage of the $\mathrm{O}-\mathrm{O}$ bond. A very unstable intermediate enzyme complex is formed before compound I and is referred in the literature as compound 0 .

additional to the conserved "core" peroxidase fold. The distal residues, His42 and Arg38 are emphasized to play critical roles in substrate stabilization, catalytic oxidation and ligand binding through an extensive hydrogen bonding network, in addition to the cooperation with the proximal His170 to accomplish the "push-pull" mechanism (14).

Although HRP has been used in a vast number of applications such as organic, enzyme assays, chemiluminescent assays, immunoassays and the treatment of waste waters, it should be pointed out that HRP is relatively less stable than other enzymes commonly used in industrial applications (e.g., hydrolases). Furthermore, the relatively 
expensive price of HRP is another obstacle preventing the usage of HRP in applications such as peroxidase-catalyzed enantioselective oxidations at the industry level. Solutions to these problems include better process management of hydrogen peroxide to avoid HRP inactivation, use of engineered or alternative enzymes with improved stability, catalytic efficiency and price points. The latter solution leads attentions to a peroxidase, soybean peroxidase (SBP), extracted from soybean seed hull, with ideally improvement from every aspect mentioned above. A brief introduction about SBP is given in Chapter 1.3.3.

\subsubsection{Cytochrome $c$ Peroxidase}

Cytochrome $c$ Peroxidase $(\mathrm{C} c \mathrm{P})$, a class I b-type heme peroxidase from yeast, is the first heme peroxidase whose X-ray crystal structure was elucidated. It is anomalous in that $\mathrm{C} c \mathrm{P}$ can utilize ferro-cytochrome $c$, a hemoprotein, as substrate, in addition to small substrates such as guaiacol, styrene, phenylhydrazine, etc. The metabolic role of $\mathrm{C} c \mathrm{P}$ is not well defined, but was suggested to relate to detoxification of hydrogen peroxide formed in the intermembrane space of mitochrondria using ferro-cytochrome $c$ as the electron donor. Despite the little interest of study from a metabolic perspective, $\mathrm{C} c \mathrm{P}$ has been extensively used as a model system and archetype in the study of peroxidase catalytic mechanism, protein electron transfer, protein engineering, and structure-function interactions.

The catalytic cycle of $\mathrm{CcP}$ is similar to that of HRP and other peroxidases, with compound I and II formation and the two-electron reduction of peroxide to water. The highly conserved distal His52 and Arg48 (figure 1.8C) play the same role as the corresponding residues in HRP through acid-base catalysis. From an analysis of the crystal structure of $\mathrm{CcP}(83)$, it was proposed that binding of the peroxide to the iron is 
facilitated by transfer of one of its hydrogens to the imidazole nitrogen of His52. It is notable that studies of $\mathrm{C} c \mathrm{P} 1.2 \AA$ crystal structure (84) revealed that $\operatorname{Arg} 48$ occupies two positions: one termed "out" and the other "in". When Arg48 occupies the "out" position, a water molecule take up the space vacated by the side chain of Arg48 and functions as the sixth axial ligand of heme iron in the resting state of the enzyme. Conversely, in Compound I, Arg48 occupies the "in" position permanently where it is able to H-bond with the ferryl oxygen atom for stabilization purpose. The major difference between $\mathrm{CcP}$ and other peroxidases is that $\mathrm{C} c \mathrm{P}$ does not form a porphyrin cation radical but instead a cationic Trp191 radical compound I (85). The $\mathrm{C} c \mathrm{P}$ compound I, is stable with a half-life of several hours in the absence of reductant (86) but rapidly returns to the ferric resting state via sequential reactions with two molecules of ferro-cytochrome c. The Trp191 residue is suggested to be essential for activity and responsible for the high stability of $\mathrm{C} c \mathrm{P}$ compound I (85).

\subsubsection{Soybean Peroxidase}

Soybean peroxidase (SBP) is a class III heme peroxidase expressed in the seed hull of soybean $\sim 20$ days after anthesis (87). Soybean peroxidase shows $57 \%$ amino acid sequence identity with the most widely studied member of this class of peroxidases, horseradish peroxidase.

As most other glycoprotein peroxidases, the native SBP encountered difficulties in obtaining suitable single crystals for X-ray diffraction. Alternatively, the noneglycosylated recombinant SBP (rSBP) produced in Escherichia coli was used for crystal structure determination (88). The rSBP and HRP-C share a very similar threedimensional structure (Figure 1.10), active-site environment (Figure 1.8 A, B), and 
catalytic mechanism. They have many structural factors in common, such as heme prosthetic group, two calcium ions, four disulfide bonds, a single tryptophan and eight glycosylation sites (89). The crystallographic structure of rSBP revealed three features of mechanistic implications: a bound Tris buffer molecule at the binding site of small phenolic substrates, a highly exposed heme edge, and the contact of one heme vinyl group with a sulfur atom from methionine.
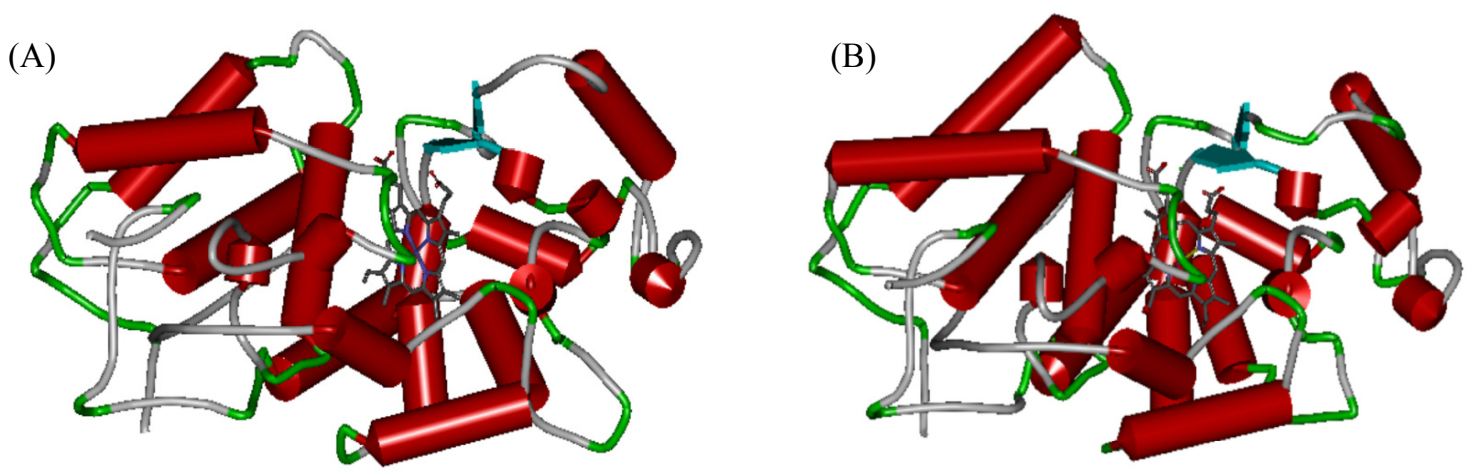

Figure 1.10 Schematic structures of (A) rSBP (PDB code: 1FHF) and (B) HRP-C (PDB code: 1ATJ) obtained from crystallographic results.

As mentioned in the end of section 3.1 of this chapter, horseradish peroxidase is not an ideal peroxidase for industrial applications but needs improvement for its activity, efficiency, stability, cost, abundance, and large-scale production. Soybean peroxidase, however, has many advantages in these aspects as an attractive alternative to HRP for industrial use. Catalytic activity of SBP is reported to be $\sim 1.5$-fold higher than that of HRP-C (90). A 20 fold higher catalytic efficiency for SBP over HRP-C at their $\mathrm{pH}$ optima was also reported (89). The thermal and kinetic stabilities of SBP are better than those of HRP (91-93). SBP has also been found to be greatly resistant to denaturation induced by high concentration of urea and guanidine hydrochloride (90). The above 
attributes together with the low cost and high abundance of SBP make it a very attractive candidate for industrial and medical uses, and in fact, SBP has been patented for a broad range of uses, such as biocatalyst, biosensor and wastewater treatment (94-106).

With most of the previous investigations focusing on the physiological property, biological functions and applications of SBP, the structural and chemical properties of the enzyme were somewhat neglected. Structural studies of SBP, other than the crystal structure of recombinant enzyme, include electronic absorption, resonance Raman, and tryptophan fluorescence studies $(90,107-109)$. It has been suggested that the higher stability of SBP compared to HRP-C may stem from the heme binding to the apoprotein, whereas a contact between heme 8-vinyl group and the sulfur atom of Met37 is observed in the SBP structure $(88,93)$.

NMR spectroscopy has been applied successfully in the active site studies of many peroxidases, such as HRP (110-114) and $\mathrm{C} c \mathrm{P}(113,115)$, which encouraged our interest in using this technique to analyze the heme cavity of SBP. Therefore, an introduction about NMR study of hemoproteins, especially heme peroxidases, is made in the following chapter.

\subsection{Nuclear Magnetic Resonance of Hemoproteins}

Heme prosthetic group is an exquisitely sensitive chromophore whose spectroscopic properties reflect a wealth of structural detail of its environment in a protein. Therefore the heme prosthetic group offers a unique probe for many biophysical techniques, such as resonance Raman, optical absorption, fluorescence and especially, nuclear magnetic resonance (NMR) spectroscopy. 
NMR plays critical roles in bioscience and biotechnology in both imaging and complete three-dimensional structure determination. Compared with X-ray crystallography, another major structural method, NMR has its advantages because it can be carried out under solution conditions without the need for protein crystallization, which can be a frustrating task for many proteins such as glycoproteins. Moreover, in NMR experiments, solution conditions such as the $\mathrm{pH}$, temperature and solute types and concentration can be easily adjusted to closely mimic a given physiological environment. Conversely, it can also be changed to any nonphysiological conditions for studies such as protein denaturation. Furthermore, in addition to protein structure determination, NMR can be applied in investigations of protein dynamic features such as domain movements, as well as studies of protein-substrate, protein-ligand, or protein-protein interactions for structural, thermodynamic and kinetic aspects.

The limitations of NMR spectroscopic study of protein structures in solution, however, are the inherent low sensitivity and poor resolution of the technique. Additionally, the slow protein tumbling increases the spin-spin lattice relaxation time $\left(\mathrm{T}_{2}\right)$ and therefore increases the resonance linewidth. The increased linewidth coupled with the number of protons in proteins result in serious spectral overlap problems.

New developments in NMR partially alleviated these problems. The NMR sensitivity and resolution have been increased by progress in both theoretical and practical spectrometer technology, which leads to an increasingly efficient utilization from the information content of NMR spectra. The effects of line broadening and spin diffusion can be diminished with deuterium substitution for hydrogen. Likewise, NMR spectra 
from uniformly or selectively labeled samples can be drastically simplified to reduce the chance of overlap.

From ${ }^{1} \mathrm{H}$ NMR of hemoprotein, the large heme ring current near the heme group can generate a small number of well-separated resonance lines in the spectra of folded, globular hemoproteins. Historically, paramagnetic metalloproteins triggered the development of protein NMR spectroscopy. This is because for the paramagnetic states of hemoproteins, additional well-resolved resonances outside of the crowded diamagnetic 0-10 ppm window are resulted from the interactions with the unpaired electrons of the heme iron. Although these hyperfine shifts only represent less than $3 \%$ of the hydrogen atoms in most hemoprotein, these signals can provide an impressive amount of information on the heme environment by revealing the paramagnetic influence on chemical shifts in an expanded chemical shift scale. The hyperfine shifts can be used to monitor electronic structural and magnetic properties of the heme. The hyperfine shifts

can also be selectively irradiated in $1 \mathrm{D}{ }^{1} \mathrm{H}$ NMR experiments to facilitate unambiguous resonance assignment in combination with isotope labeling of heme prosthetic groups. Since the paramagnetism offers more valuable NMR information of hemoproteins, I will further focus on introducing hemoproteins NMR under paramagnetic conditions.

\subsubsection{NMR Information Content}

The general goal of NMR is to detect and assign the heme and protein signals as much as possible, and to arrange the heme and the amino acid residues in their proper spatial relationships. The information content directly given by an NMR spectrum includes chemical shifts and spin relaxations. After calibration to some convenient reference, the observed chemical shift for an arbitrary nucleus in a paramagnetic 
hemoprotein can be described as the sum of the diamagnetic shift for the nucleus in the absence of unpaired electrons and the net paramagnetic hyperfine shift caused by the unpaired electron spins on the iron (116). The latter shift can be further divided into two contributions: the contact shift which arises from partial unpaired spin density delocalization from the paramagnetic heme iron onto a ligand nucleus, and the dipolar shift caused by a dipolar or through-space interaction between any residue nuclei and the unpaired spin on the iron. The paramagnetic hyperfine shift is temperature dependent and will be discussed in Chapter 1.4.2.

Different physical processes are responsible for the spin relaxation of a nucleus. According to the corresponding relationship between the nuclear spin magnetization vector and the external magnetic field, two principal relaxation processes are defined as $T_{1}$ (spin-lattice relaxation time, longitudinal) and $T_{2}$ (spin-spin relaxation time, transverse). The relative paramagnetic contribution to the intrinsic $T_{1}$ for two nonequivalent nuclei, $i, j$ on residues not bonded to the heme iron follows the relationship of Equation 7:

$$
r_{i} / r_{j}=\left[\mathrm{T}_{1 i} / \mathrm{T}_{1 j}\right]^{1 / 6}
$$

with which the relative distances of the two nuclei to the iron, $r_{i}$ and $r_{j}$ can be determined. Therefore, it is convenient to indicate the varying effect of paramagnetic relaxivity throughout a protein in relevant of the heme iron.

\subsubsection{Temperature Dependence of Hyperfine Shifts}

The temperature dependence of hyperfine shifts is complicated depending on different oxidation/spin state of the heme iron. Under a simplest ideal situation for a well- 
isolated spin state with no zero-field splitting, the paramagnetic susceptibility tensor $\chi$ obeys Curie's law (Equation 8):

$$
\chi=\frac{\mu_{0} \mathrm{~N}_{\mathrm{A}} \mathrm{g}^{2} \beta^{2} \mathrm{~S}(\mathrm{~S}+1)}{3 k \mathrm{~T}}
$$

where $\mu_{0}$ is the permeability of vacuum, $\mathrm{N}_{\mathrm{A}}$ is Avogadro's number, $\beta$ is the Bohr magneton, $\mathrm{g}$ is the spectroscopic splitting tensor, $k$ is the Boltzmann constant and $\mathrm{T}$ is the absolute temperature. The hyperfine sift of a ligand nucleus $j, \delta_{\mathrm{hf}}^{j}$ can therefore be simplified as Equation 9:

$$
\delta_{\mathrm{hf}}^{j}=(\mathrm{A} / \mathrm{h})^{j} \frac{\mathrm{g} \beta \mathrm{S}(\mathrm{S}+1)}{3 k \mathrm{~T}(\gamma / 2 \pi)}
$$

where $(\mathrm{A} / \mathrm{h})^{j}$ is the hyperfine coupling constant for nucleus $j$ in $\mathrm{Hz}$, and $\gamma$ is the nuclear magnetogyric ratio. Therefore $\delta_{\mathrm{hf}}$ will exhibit strict Curie behavior (linear in 1/T with zero intercept at $\mathrm{T} \rightarrow \infty)$. However, with different electronic structure of the heme iron, the hyperfine shifts may exhibit hyper-Curie (intercept at $\mathrm{T} \rightarrow \infty<0$ ), hypo-Curie (intercept at $\mathrm{T} \rightarrow \infty>0$ ), or even anti-Curie (intercept at $\mathrm{T} \rightarrow \infty>\delta_{\mathrm{hf}}$ ) temperature behaviors.

For low-spin (LS) ferriheme group of hemoproteins, the four methyl groups are divided into the $1-\mathrm{CH}_{3}, 5-\mathrm{CH}_{3}$ and the $3-\mathrm{CH}_{3}, 8-\mathrm{CH}_{3}$ pairs because of their relative shifted positions shown on the NMR spectrum. Normally the methyl groups exhibit two strongly $(\sim 30 \mathrm{ppm})$ and two weakly $(\sim 5 \mathrm{ppm})$ low-field contact shifted resonances with either the $1-\mathrm{CH}_{3}, 5-\mathrm{CH}_{3}$ or the $3-\mathrm{CH}_{3}, 8-\mathrm{CH}_{3}$ shown together in the low-field region, depending on the orientation of the proximal ligand with respect to the heme plane. It is generally observed that the two strongly shifted heme methyls exhibit strong hyper-Curie and the other two weakly shifted methyls exhibit anti-Curie behavior (117-124). For the 
axial His in peroxidase- $\mathrm{CN}$ complexes, the contact shifts normally show strong hyperCurie behavior.

\subsubsection{Nuclear Overhauser Effect}

The nuclear Overhauser effect (NOE) is a manifestation of the dipolar interactions between different nuclei. Although the NOE is a common phenomenon for all combinations of closely spaced nuclear spins, ${ }^{1} \mathrm{H}-{ }^{1} \mathrm{H}$ NOEs are of prime interest for structural studies because it is related to the "through-space" distance between a pair of hydrogens that are in proximity to each other without the need for spin-coupling through covalent linkage. The intensity of the NOE is in first approximation proportional to $1 / \mathrm{r}^{6}$, in which $\mathrm{r}$ is the distance between the protons, and normally for two adjacent protons, a NOE signal is only observed if their distance is smaller than $5 \AA$.

However, the NOE is highly restricted in large proteins or paramagnetic systems because spin diffusion in large systems obscures selective primary NOEs. This problem can be solved by saturating a paramagnetically relaxed nucleus $i$ for a very short time $t$, yielding $\eta_{i-j}$, the NOE to proton $j$, becomes independent of the intrinsic relaxation time of the nucleus as shown in equation 10 :

$$
\eta_{i-j}(t)=\sigma_{i j} t
$$

where $\sigma_{i j}$ is the cross-relaxation rate. The above equation indicates that NOEs should be applicable in paramagnetic systems if resonances can be saturated quickly. Therefore, even steady-state NOEs can be observed in large paramagnetic hemoprotein systems where $\sigma_{i j}$ is large, allowing unambiguous assignment of hyperfine signals in hemoproteins at different oxidation states. The paramagnetism diminishes the NOEs but 
at the same time weakens the spin diffusion, so that the resulting NOEs reflect mostly an internuclear distance of $\sim 3 \AA(125-126)$.

The 1D NOE and 2D NOESY experiments are both useful and essential to the determination of protein structure. The $1 \mathrm{D}$ NOE is relatively more sensitive and selective for hyperfine shifts but is less effective for signals buried in the diamagnetic envelope. In contrast, the NOESY spectrum offers information of the entire ${ }^{1} \mathrm{H}$ NMR spectrum of a protein with greatly improved resolution of the individual peaks, which is especially valuable for resonances in the crowded diamagnetic window.

A series of NMR studies on $\mathrm{HRP}-\mathrm{CN}^{-}$complex using $1 \mathrm{D}$ steady state NOE and 2D NOESY (110-114, 126-133), combined with isotope labeling method, have provided unambiguous heme assignments, identification of catalytic residues, and information on the spatial disposition of such residues in the active site. Results from HRP have been used as the benchmark and model systems for developing successful approaches to the NMR studies of other paramagnetic hemoproteins.

\subsubsection{Low-Spin Cyanide Complex of Hemoprotein}

The low-spin ferric $(S=1 / 2)$ state of the heme iron is the structurally most informative oxidation/spin state, in which all the electrons are, to first order, in $\pi$-bonding or nonbonding iron orbitals. Therefore, electron spin can only be delocalized into $\pi$ ligand orbitals. For NMR spectra of low-spin state hemoproteins, both linewidth (determined by the electronic spin relaxation) and hyperfine shift dispersion (determined by the magnetic anisotropy) depend critically on the axial ligand because of the orbital splitting gap. In hemoproteins having a histidine proximal ligand, the cyanide inhibited complexes can

provide well-resolved ${ }^{1} \mathrm{H}$ NMR spectrum with the narrowest lines and the largest 
magnetic anisotropies by utilizing $\mathrm{CN}^{-}$as the distal ligand. In addition to affecting the side chains on heme, the $\mathrm{His} / \mathrm{CN}^{-}$coordination also produces strong spectral dispersion for nonligated amino acid residues around the heme iron because of significant dipolar shifts.

However, in the case of cyanide inhibited hemoproteins using cysteine as the axial ligand, the $\mathrm{Cys} / \mathrm{CN}^{-}$coordination causes less efficient iron electron spin relaxation, and hence results in larger linewidths, much smaller hyperfine-shift dispersion, and strongly relaxed heme signals, making it much more difficult to extract useful information from the NMR results for nuclei in proximity to the heme iron (123).

In conclusion, with a combination of model compounds or proteins, isotope labeling, differential relaxation study and proper 1D/2D NMR methods, resonances from the heme, the axial ligands, and amino acids in the heme cavity can be assigned. The resulted full scope of the NMR investigation can provide valuable information about the threedimensional structure of the active site of the hemoprotein in solution.

\subsection{References}

1. Chapman, S., Daff, S., and Munro, A. (1997) Heme: The most versatile redox centre in biology?, In Metal Sites in Proteins and Models (Hill, H., Sadler, P., and Thomson, A., Eds.), pp 39-70, Springer Berlin / Heidelberg.

2. Bertini, I., Sigel, A., and Sigel, H. (2001) Handbook on metalloproteins, Marcel Dekker, New York.

3. Lu, Y., Berry, S. M., and Pfister, T. D. (2001) Engineering Novel Metalloproteins: Design of Metal-Binding Sites into Native Protein Scaffolds, Chemical Reviews 101, 3047-3080.

4. Malatesta, F., Antonini, G., Sarti, P., and Brunori, M. (1995) Structure and function of a molecular machine: cytochrome c oxidase, Biophysical Chemistry $54,1-33$. 
5. Scott, R. A., and Mauk, A. G. (1996) Cytochrome c: A multidisciplinary approach, University Science Books, Sausalito.

6. Silvestrini, M. C., Falcinelli, S., Ciabatti, I., Cutruzzolà, F., and Brunori, M. (1994) Pseudomonas aeruginosa nitrite reductase (or cytochrome oxidase): An overview, Biochimie 76, 641-654.

7. La Mar, G. N., Toi, H., and Krishnamoorthi, R. (1984) Proton NMR investigation of the rate and mechanism of heme rotation in sperm whale myoglobin: evidence for intramolecular reorientation about a heme two-fold axis, Journal of the American Chemical Society 106, 6395-6401.

8. GR, M., and GW, P. (1990) Cytochromes c: Evolutionary, structural and physicochemical aspects, Springer-Verlag, Berlin Heidelberg, New York.

9. Sligar, S. G., Makris, T. M., and Denisov, I. G. (2005) Thirty years of microbial P450 monooxygenase research: Peroxo-heme intermediates--The central bus station in heme oxygenase catalysis, Biochemical and Biophysical Research Communications 338, 346-354.

10. Garfinkel, D. (1958) Studies on pig liver microsomes. I. Enzymic and pigment composition of different microsomal fractions, Archives of Biochemistry and Biophysics 77, 493-509.

11. Klingenberg, M. (1958) Pigments of rat liver microsomes, Archives of Biochemistry and Biophysics 75, 376-386.

12. Guengerich, F. P. (1991) Reactions and significance of cytochrome P-450 enzymes, Journal of Biological Chemistry 266, 10019-10022.

13. Hasemann, C. A., Kurumbail, R. G., Boddupalli, S. S., Peterson, J. A., and Deisenhofer, J. (1995) Structure and function of cytochromes P450:a comparative analysis of three crystal structures, Structure (London, England : 1993) 3, 41-62.

14. Sono, M., Roach, M. P., Coulter, E. D., and Dawson, J. H. (1996) HemeContaining Oxygenases, Chemical Reviews 96, 2841-2888.

15. van den Brink, H. M., van Gorcom, R. F. M., van den Hondel, C. A. M. J. J., and Punt, P. J. (1998) Cytochrome P450 Enzyme Systems in Fungi, Fungal Genetics and Biology 23, 1-17.

16. Wong, L.-L. (1998) Cytochrome P450 monooxygenases, Current Opinion in Chemical Biology 2, 263-268.

17. Denisov, I. G., Makris, T. M., Sligar, S. G., and Schlichting, I. (2005) Structure and Chemistry of Cytochrome P450, Chemical Reviews 105, 2253-2278. 
18. Mansuy, D. (2007) A brief history of the contribution of metalloporphyrin models to cytochrome P450 chemistry and oxidation catalysis, Comptes Rendus Chimie $10,392-413$.

19. Mansuy, D. (1998) The great diversity of reactions catalyzed by cytochromes P450, Comparative Biochemistry and Physiology Part C: Pharmacology, Toxicology and Endocrinology 121, 5-14.

20. Bernhardt, R. (2006) Cytochromes P450 as versatile biocatalysts, Journal of Biotechnology 124, 128-145.

21. Omura, T. (2010) Structural diversity of cytochrome P450 enzyme system, Journal of Biochemistry 147, 297-306.

22. Guengerich, F. P. (2001) Common and Uncommon Cytochrome P450 Reactions Related to Metabolism and Chemical Toxicity, Chemical Research in Toxicology 14, 611-650.

23. Montellano, P. (2005) Cytochrome P450: structure, mechanism, and biochemistry, 3rd ed., Kluwer Academic/Plenum Publishers.

24. Ioannides, C. (1996) Cytochromes P450: metabolic and toxicological aspects, CRC Press.

25. Lechner, M. (1994) Cytochrome P450: biochemistry, biophysics, and molecular biology : 8th international conference, Lisbon, Portugal, 24-28 October 1993, John Libbey Eurotext.

26. Lewis, D. (1996) Cytochromes P450: structure, function and mechanism, Taylor \& Francis.

27. Lewis, D. (2001) Guide to cytochromes P450: structure and function, Taylor \& Francis.

28. Ioannides, C. (2008) Cytochromes P450: role in the metabolism and toxicity of drugs and other xenobiotics, RSC Pub.

29. Nelson, D. R. (2009) The cytochrome p450 homepage, Human Genomics 4, 5965.

30. Newcomb, M., Zhang, R., Chandrasena, R. E. P., Halgrimson, J. A., Horner, J. H., Makris, T. M., and Sligar, S. G. (2006) Cytochrome P450 Compound I, Journal of the American Chemical Society 128, 4580-4581.

31. Katagiri, M., Ganguli, B. N., and Gunsalus, I. C. (1968) A Soluble Cytochrome P450 Functional in Methylene Hydroxylation, Journal of Biological Chemistry 243, 3543-3546. 
32. Poulos, T. L., Finzel, B. C., Gunsalus, I. C., Wagner, G. C., and Kraut, J. (1985) The 2.6-A crystal structure of Pseudomonas putida cytochrome P-450, Journal of Biological Chemistry 260, 16122-16130.

33. Poulos, T. L., Finzel, B. C., and Howard, A. J. (1987) High-resolution crystal structure of cytochrome P450cam, Journal of Molecular Biology 195, 687-700.

34. Shaw, P. D., and Hager, L. P. (1959) An Enzymatic Chlorination Reaction, Journal of the American Chemical Society 81, 1011-1012.

35. Lowell P. Hager, D. R. M., Frederick S. Brown and Horst Eberwein (1966) Chloroperoxidase II. Utilization of Halogen Anions, Journal of Biological Chemistry 241, 1769-1777.

36. Dawson, J. H., and Sono, M. (1987) Cytochrome P-450 and chloroperoxidase: thiolate-ligated heme enzymes. Spectroscopic determination of their active-site structures and mechanistic implications of thiolate ligation, Chemical Reviews 87, 1255-1276.

37. Griffin, B. W. (1991) Peroxidases in Chemistry and Biology, CRC Press, Boca Raton, FL.

38. Dexter, A. F., Lakner, F. J., Campbell, R. A., and Hager, L. P. (1995) Highly Enantioselective Epoxidation of 1,1-Disubstituted Alkenes Catalyzed by Chloroperoxidase, Journal of the American Chemical Society 117, 6412-6413.

39. Zaks, A., and Dodds, D. R. (1995) Chloroperoxidase-catalyzed asymmetric oxidations: substrate specificity and mechanistic study, Journal of the American Chemical Society 117, 10419-10424.

40. Hu, S., and Hager, L. P. (1999) Highly Enantioselective Propargylic Hydroxylations Catalyzed by Chloroperoxidase, Journal of the American Chemical Society 121, 872-873.

41. G L Kedderis, D. R. K. a. P. F. H. (1980) N-Demethylation reactions catalyzed by chloroperoxidase, Journal of Biological Chemistry 255, 10174-10182.

42. Colonna S., G. N., Carrea G., Pasta P. (1992) Horseradish peroxidase catalysed sulfoxidation is enantioselective Journal of the Chemical Society, Chemical Communications 357-358.

43. Lindborg, J. T., Annukka; Kanerva, Liisa T. (2009) Chemoselective chloroperoxidase-catalyzed oxidation of hexen-1-ols, Biocatalysis and Biotransformation 27, 204-210. 
44. Hager, L. P., Lakner, F. J., and Basavapathruni, A. (1998) Chiral synthons via chloroperoxidase catalysis, Journal of Molecular Catalysis B: Enzymatic 5, 95101.

45. Allain, E. J., Hager, L. P., Deng, L., and Jacobsen, E. N. (1993) Highly enantioselective epoxidation of disubstituted alkenes with hydrogen peroxide catalyzed by chloroperoxidase, Journal of the American Chemical Society 115, 4415-4416.

46. Dugad, L. B., Wang, X., Wang, C. C., Lukat, G. S., and Goff, H. M. (1992) Proton nuclear Overhauser effect study of the heme active site structure of chloroperoxidase, Biochemistry 31, 1651-1655.

47. Kincaid, S. H. a. J. R. (1993) Heme active-site structural characterization of chloroperoxidase by resonance Raman spectroscopy, Journal of Biological Chemistry 268, 6189-6193.

48. Dawson, J. H., Trudell, J. R., Barth, G., Linder, R. E., Bunnenberg, E., Djerassi, C., Chiang, R., and Hager, L. P. (1976) Magnetic circular dichroism studies. 44. Chloroperoxidase. Evidence for P-450 type heme environment from magnetic circular dichroism spectroscopy, Journal of the American Chemical Society 98, 3709-3710.

49. Cramer, S. P., Dawson, J. H., Hodgson, K. O., and Hager, L. P. (1978) Studies of the ferric forms of cytochrome P-450 and chloroperoxidase by extended X-ray absotption fine structure. Characterization of the iron-nitrogen and iron-sulfur distances, Journal of the American Chemical Society 100, 7282-7290.

50. Sundaramoorthy, M., Terner, J., and Poulos, T. L. (1995) The crystal structure of chloroperoxidase: a heme peroxidase-cytochrome P450 functional hybrid, Structure 3, 1367-1378.

51. Palcic, M. M., Rutter, R., Araiso, T., Hager, L. P., and Dunford, H. B. (1980) Spectrum of chloroperoxidase compound I, Biochemical and Biophysical Research Communications 94, 1123-1127.

52. Kenigsberg, P., Fang, G.-H., and Hager, L. P. (1987) Post-translational modifications of chloroperoxidase from Caldariomyces fumago, Archives of Biochemistry and Biophysics 254, 409-415.

53. Hager, L. P. (2010) A Lifetime of Playing with Enzymes, Journal of Biological Chemistry 285, 14852-14860.

54. Dexter, A. F., and Hager, L. P. (1995) Transient Heme N-Alkylation of Chloroperoxidase by Terminal Alkenes and Alkynes, Journal of the American Chemical Society 117, 817-818. 
55. Debrunner, P., Dexter, A., Schulz, C., Xia, Y.-M., and Hager, L. P. (1996) Mossbauer and electron paramagnetic resonance studies of chloroperoxidase following mechanism-based inactivation with allylbenzene, Proceedings of the National Academy of Sciences 93, 12791-12798.

56. De Matteis, F. (1971) Loss of haem in rat liver caused by the porphyrogenic agent 2-allyl-2-isopropylacetamide, Biochemical Journal 124, 767-777.

57. Levin, W., Sernatinger, E., Jacobson, M., and Kuntzman, R. (1972) Destruction of Cytochrome P450 by Secobarbital and Other Barbiturates Containing Allyl Groups, Science 176, 1341-1343.

58. Kent L. Kunze, B. L. K. M., Conrad Wheeler, Hal S. Beilan, and Paul R. Ortiz de Montellano. (1983) The cytochrome P-450 active site. Regiospecificity of prosthetic heme alkylation by olefins and acetylenes., Journal of Biological Chemistry 258, 4202-4207.

59. Blobaum, A. L., Kent, U. M., Alworth, W. L., and Hollenberg, P. F. (2004) Novel Reversible Inactivation of Cytochrome P450 2E1 T303A by tert-Butyl Acetylene: The Role of Threonine 303 in Proton Delivery to the Active Site of Cytochrome P450 2E1, Journal of Pharmacology and Experimental Therapeutics 310, 281290.

60. Lu, Y., Kent, U. M., Wang, S., and Hollenberg, P. F. (2004) Formation of a novel reversible cytochrome P450 spectral intermediate: role of threonine 303 in P450 2E1 inactivation, Biochemistry 43, 11942-11952.

61. Lee, H.-I., Dexter, A. F., Fann, Y.-C., Lakner, F. J., Hager, L. P., and Hoffman, B. M. (1997) Structure of the Modified Heme in Allylbenzene-Inactivated Chloroperoxidase Determined by Q-Band CW and Pulsed ENDOR, Journal of the American Chemical Society 119, 4059-4069.

62. Adam, W., Lazarus, M., Saha-Möller, C., Weichold, O., Hoch, U., Häring, D., and Schreier, P. (1999) Biotransformations with Peroxidases, In Biotransformations (Faber, K., Ed.), pp 73-108, Springer Berlin / Heidelberg.

63. van Deurzen, M. P. J., van Rantwijk, F., and Sheldon, R. A. (1997) Selective oxidations catalyzed by peroxidases, Tetrahedron 53, 13183-13220.

64. Welinder, K. G. (1992) Superfamily of plant, fungal and bacterial peroxidases, Current Opinion in Structural Biology 2, 388-393.

65. Veitch, N. C., and Smith, A. T. (2000) Horseradish peroxidase, In Advances in Inorganic Chemistry, pp 107-162, Academic Press.

66. Wagner, M., and Nicell, J. A. (2002) Detoxification of phenolic solutions with horseradish peroxidase and hydrogen peroxide, Water Research 36, 4041-4052. 
67. Velde, F. v. d., Rantwijk, F. v., and Sheldon, R. A. (2001) Improving the catalytic performance of peroxidases in organic synthesis, Trends in Biotechnology 19, 7380.

68. Dotsikas, Y., and Loukas, Y. L. (2004) Employment of 4-(1-imidazolyl)phenol as a luminol signal enhancer in a competitive-type chemiluminescence immunoassay and its comparison with the conventional antigen-horseradish peroxidase conjugate-based assay, Analytica Chimica Acta 509, 103-109.

69. Azevedo, A. M., Martins, V. C., Prazeres, D. M. F., Vojinovic, V., Cabral, J. M. S., and Fonseca, L. P. (2003) Horseradish peroxidase: a valuable tool in biotechnology, In Biotechnology Annual Review (El-Gewely, M. R., Ed.), pp 199247, Elsevier.

70. Veitch, N. C. (2004) Horseradish peroxidase: a modern view of a classic enzyme, Phytochemistry 65, 249-259.

71. Morawski, B., Lin, Z., Cirino, P., Joo, H., Bandara, G., and Arnold, F. H. (2000) Functional expression of horseradish peroxidase in Saccharomyces cerevisiae and Pichia pastoris, Protein Engineering 13, 377-384.

72. Tupper, J., Tozer, G. M., and Dachs, G. U. (2004) Use of horseradish peroxidase for gene-directed enzyme prodrug therapy with paracetamol, Br J Cancer 90, $1858-1862$.

73. Haschke, R. H., and Friedhoff, J. M. (1978) Calcium-related properties of horseradish peroxidase, Biochemical and Biophysical Research Communications 80, 1039-1042.

74. Howes, B. D., Feis, A., Raimondi, L., Indiani, C., and Smulevich, G. (2001) The Critical Role of the Proximal Calcium Ion in the Structural Properties of Horseradish Peroxidase, Journal of Biological Chemistry 276, 40704-40711.

75. Smith, A. T., Santama, N., Dacey, S., Edwards, M., Bray, R. C., Thorneley, R. N., and Burke, J. F. (1990) Expression of a synthetic gene for horseradish peroxidase $\mathrm{C}$ in Escherichia coli and folding and activation of the recombinant enzyme with $\mathrm{Ca} 2+$ and heme, Journal of Biological Chemistry 265, 13335-13343.

76. Filizola, M., and Loew, G. H. (1999) Role of Protein Environment in Horseradish Peroxidase Compound I Formation: Molecular Dynamics Simulations of Horseradish Peroxidase-HOOH Complex, Journal of the American Chemical Society 122, 18-25.

77. Berglund, G. I., Carlsson, G. H., Smith, A. T., Szoke, H., Henriksen, A., and Hajdu, J. (2002) The catalytic pathway of horseradish peroxidase at high resolution, Nature 417, 463-468. 
78. Derat, E., and Shaik, S. (2006) The Poulos-Kraut Mechanism of Compound I Formation in Horseradish Peroxidase: A QM/MM Study, The Journal of Physical Chemistry B 110, 10526-10533.

79. Schulz, C. E., Rutter, R., Sage, J. T., Debrunner, P. G., and Hager, L. P. (1984) Moessbauer and electron paramagnetic resonance studies of horseradish peroxidase and its catalytic intermediates, Biochemistry 23, 4743-4754.

80. Penner-Hahn, J. E., Smith Eble, K., McMurry, T. J., Renner, M., Balch, A. L., Groves, J. T., Dawson, J. H., and Hodgson, K. O. (1986) Structural characterization of horseradish peroxidase using EXAFS spectroscopy. Evidence for $\mathrm{Fe}=\mathrm{O}$ ligation in compounds I and II, Journal of the American Chemical Society 108, 7819-7825.

81. Makino, R., Uno, T., Nishimura, Y., Iizuka, T., Tsuboi, M., and Ishimura, Y. (1986) Coordination structures and reactivities of compound II in iron and manganese horseradish peroxidases. A resonance Raman study, Journal of Biological Chemistry 261, 8376-8382.

82. Gajhede, M., Schuller, D. J., Henriksen, A., Smith, A. T., and Poulos, T. L. (1997) Crystal structure of horseradish peroxidase $\mathrm{C}$ at 2.15 A resolution, Nature Structural \& Molecular Biology 4, 1032-1038.

83. Poulos, T. L., and Kraut, J. (1980) The stereochemistry of peroxidase catalysis, Journal of Biological Chemistry 255, 8199-8205.

84. Bonagura, C. A., Bhaskar, B., Shimizu, H., Li, H., Sundaramoorthy, M., McRee, D. E., Goodin, D. B., and Poulos, T. L. (2003) High-Resolution Crystal Structures and Spectroscopy of Native and Compound I Cytochrome c Peroxidase $\dagger$, Biochemistry 42, 5600-5608.

85. Mauro, J. M., Fishel, L. A., Hazzard, J. T., Meyer, T. E., Tollin, G., Cusanovich, M. A., and Kraut, J. (1988) Tryptophan-191 .fwdarw. phenylalanine, a proximalside mutation in yeast cytochrome c peroxidase that strongly affects the kinetics of ferrocytochrome c oxidation, Biochemistry 27, 6243-6256.

86. Erman, J. E., and Yonetani, T. (1975) A kinetic study of the endogenous reduction of the oxidized sites in the primary cytochrome c peroxidase-hydrogen peroxide compound, Biochimica et Biophysica Acta (BBA) - Protein Structure $393,350-357$.

87. Gillikin, J. W., and Graham, J. S. (1991) Purification and Developmental Analysis of the Major Anionic Peroxidase from the Seed Coat of Glycine max, Plant Physiol. 96, 214-220. 
88. Henriksen, A., Mirza, O., Indiani, C., Teilum, K., Smulevich, G., Welinder, K. G., and Gajhede, M. (2001) Structure of soybean seed coat peroxidase: A plant peroxidase with unusual stability and haem-apoprotein interactions, Protein Science 10, 108-115.

89. Kamal, J. K. A., and Behere, D. V. (2003) Activity, stability and conformational flexibility of seed coat soybean peroxidase, Journal of Inorganic Biochemistry 94 , 236-242.

90. Amisha Kamal, J. K., and Behere, D. V. (2001) Steady-State and Picosecond Time-Resolved Fluorescence Studies on Native and Apo Seed Coat Soybean Peroxidase, Biochemical and Biophysical Research Communications 289, 427433.

91. McEldoon, J. P., and Dordick, J. S. (1996) Unusual Thermal Stability of Soybean Peroxidase, Biotechnology Progress 12, 555-558.

92. Kamal, J. K. A., and Behere, D. V. (2002) Thermal and Conformational Stability of Seed Coat Soybean Peroxidase, Biochemistry 41, 9034-9042.

93. Kamal, J. K. A., and Behere, D. V. (2008) Kinetic stabilities of soybean and horseradish peroxidases, Biochemical Engineering Journal 38, 110-114.

94. Kenausis, G., Chen, Q., and Heller, A. (1997) Electrochemical Glucose and Lactate Sensors Based on "Wired" Thermostable Soybean Peroxidase Operating Continuously and Stably at $37^{\circ} \mathrm{C}$, Analytical Chemistry 69, 1054-1060.

95. Wang, B., Li, B., Wang, Z., Xu, G., Wang, Q., and Dong, S. (1999) Sol-Gel Thin-Film Immobilized Soybean Peroxidase Biosensor for the Amperometric Determination of Hydrogen Peroxide in Acid Medium, Analytical Chemistry 71, 1935-1939.

96. Ramos, M. C., Torijas, M. C., and Díaz, A. N. (2001) Enhanced chemiluminescence biosensor for the determination of phenolic compounds and hydrogen peroxide, Sensors and Actuators B: Chemical 73, 71-75.

97. Patapas, J., Al-Ansari, M. M., Taylor, K. E., Bewtra, J. K., and Biswas, N. (2007) Removal of dinitrotoluenes from water via reduction with iron and peroxidasecatalyzed oxidative polymerization: A comparison between Arthromyces ramosus peroxidase and soybean peroxidase, Chemosphere 67, 1485-1491.

98. Gómez, J. L., Bódalo, A., Gómez, E., Hidalgo, A. M., Gómez, M., and Murcia, M. D. (2008) A transient design model of a continuous tank reactor for removing phenol with immobilized soybean peroxidase and hydrogen peroxide, Chemical Engineering Journal 145, 142-148. 
99. Al-Ansari, M. M., Steevensz, A., Al-Aasm, N., Taylor, K. E., Bewtra, J. K., and Biswas, N. (2009) Soybean peroxidase-catalyzed removal of phenylenediamines and benzenediols from water, Enzyme and Microbial Technology 45, 253-260.

100. Gomez, M., Matafonova, G., Gomez, J. L., Batoev, V., and Christofi, N. (2009) Comparison of alternative treatments for 4-chlorophenol removal from aqueous solutions: Use of free and immobilized soybean peroxidase and $\mathrm{KrCl}$ excilamp, Journal of Hazardous Materials 169, 46-51.

101. Jiménez, L., Parra, M., Tomás, V., Martinez-Gutiérrez, R., García-Cánovas, F., and Tudela, J. (2009) Indigo carmine biodegradation catalysed by soybean peroxidase, New Biotechnology 25, S161-S161.

102. Parra, M., Martínez-Ruíz, J., Tomás, V., Martínez-Gutiérrez, R., García-Cánovas, F., and Tudela, J. (2009) Optimization of the soybean peroxidase catalysed biodegradation of 2,4,6-trichlorophenol, New Biotechnology 25, S160-S161.

103. Shi, L., Liu, X., Niu, W., Li, H., Han, S., Chen, J., and Xu, G. (2009) Hydrogen peroxide biosensor based on direct electrochemistry of soybean peroxidase immobilized on single-walled carbon nanohorn modified electrode, Biosensors and Bioelectronics 24, 1159-1163.

104. Vdovenko, M. M., Ciana, L. D., and Sakharov, I. Y. (2009) 3-(10'Phenothiazinyl)propane-1-sulfonate is a potent enhancer of soybean peroxidaseinduced chemiluminescence, Analytical Biochemistry 392, 54-58.

105. Vdovenko, M. M., Zubkov, A. V., Kuznetsova, G. I., Ciana, L. D., Kuzmina, N. S., and Sakharov, I. Y. (2010) Development of ultra-sensitive soybean peroxidase-based CL-ELISA for the determination of human thyroglobulin, Journal of Immunological Methods 362, 127-130.

106. Marchis, T., Avetta, P., Bianco-Prevot, A., Fabbri, D., Viscardi, G., and Laurenti, E. (2011) Oxidative degradation of Remazol Turquoise Blue G 133 by soybean peroxidase, Journal of Inorganic Biochemistry 105, 321-327.

107. Bedard, P., and Mabrouk, P. A. (1997) Resonance Raman Spectroscopy of Soybean Peroxidase, Biochemical and Biophysical Research Communications 240, 65-67.

108. Nissum, M., Feis, A., and Smulevich, G. (1998) Characterization of soybean seed coat peroxidase: Resonance Raman evidence for a structure-based classification of plant peroxidases, Biospectroscopy 4, 355-364.

109. Welinder, K. G., and Larsen, Y. B. (2004) Covalent structure of soybean seed coat peroxidase, Biochimica et Biophysica Acta (BBA) - Proteins \& Proteomics 1698, 121-126. 
110. Thanabal, V., DeRopp, J. S., and La Mar, G. N. (1987) Proton NMR study of the electronic and molecular structure of the heme cavity in horseradish peroxidase. Complete heme resonance assignments based on saturation transfer and nuclear Overhauser effects, Journal of the American Chemical Society 109, 265-272.

111. Thanabal, V., De Ropp, J. S., and La Mar, G. N. (1988) Proton NMR characterization of the catalytically relevant proximal and distal hydrogenbonding networks in ligated resting state horseradish peroxidase, Journal of the American Chemical Society 110, 3027-3035.

112. Thanabal, V., La Mar, G. N., and De Ropp, J. S. (1988) A nuclear Overhauser effect study of the heme crevice in the resting state and compound I of horseradish peroxidase: evidence for cation radical delocalization to the proximal histidine, Biochemistry 27, 5400-5407.

113. Banci, L., Bertini, I., Turano, P., Ferrer, J. C., and Mauk, A. G. (1991) Comparative proton NMR study of ferric low-spin cytochrome $\mathrm{c}$ peroxidase and horseradish peroxidase, Inorganic Chemistry 30, 4510-4516.

114. De Ropp, J. S., Yu, L. P., and La Mar, G. N. (1991) 2D NMR of paramagnetic metalloenzymes: Cyanide-inhibited horseradish peroxidase, Journal of Biomolecular NMR 1, 175-190.

115. Satterlee, J. D., Erman, J. E., LaMar, G. N., Smith, K. M., and Langry, K. C. (1983) Assignment of hyperfine-shifted resonances in low-spin forms of cytochrome c peroxidase by reconstitutions with deuterated hemins, Journal of the American Chemical Society 105, 2099-2104.

116. Bertini, I., and Luchinat, C. (1996) Chapter 1 Introduction, Coordination Chemistry Reviews 150, 1-28.

117. McDonald, C. C., and Phillips, W. E. (1973) Proton magnetic resonance studies of horse cytochrome c, Biochemistry 12,3170-3186.

118. Chao, Y.-Y. H., Bersohn, R., and Aisen, P. (1979) Proton NMR and ESR studies of oxidized cytochrome c551 from Pseudomonas aeruginosa, Biochemistry 18, $774-779$.

119. Smith, G. M. (1979) Proton nuclear magnetic resonance studies of Rhodospirillum rubrum cytochrome c2, Biochemistry 18, 1628-1634.

120. Timkovich, R., Cork, M. S., and Taylor, P. V. (1984) Proton NMR spectroscopy of Paracoccus denitrificans cytochrome c-550, Biochemistry 23, 3526-3533.

121. Turner, D. L. (1993) Evaluation of 13C and 1H Fermi contact shifts in horse cytochrome $\mathrm{c}$ The origin of the anti-Curie effect, European Journal of Biochemistry 211, 563-568. 
122. Zhang, W., La Mar, G. N., and Gersonde, K. (1996) Solution 1H-NMR Structure of the Heme Cavity in the Low-Affinity State for the Allosteric Monomeric Cyano-Met Hemoglobins from Chironomus thummi thummi, European Journal of Biochemistry 237, 841-853.

123. Kolczak, U., Han, C., Sylvia, L. A., and La Mar, G. N. (1997) Approaches to the Solution NMR Characterization of Active Sites for $65 \mathrm{kDa}$ Tetrameric Hemoglobins in the Paramagnetic Cyanomet State, Journal of the American Chemical Society 119, 12643-12654.

124. Banci, L., Bertini, I., Luchinat, C., Pierattelli, R., Shokhirev, N. V., and Walker, F. A. (1998) Analysis of the Temperature Dependence of the 1H and 13C Isotropic Shifts of Horse Heart Ferricytochrome c: Explanation of Curie and Anti-Curie Temperature Dependence and Nonlinear Pseudocontact Shifts in a Common TwoLevel Framework, Journal of the American Chemical Society 120, 8472-8479.

125. Kalk, A., and Berendsen, H. J. C. (1976) Proton magnetic relaxation and spin diffusion in proteins, Journal of Magnetic Resonance (1969) 24, 343-366.

126. Thanabal, V., De Ropp, J. S., and La Mar, G. N. (1986) Determination of vinyl orientation in resting state and compound I of horseradish peroxidase by the proton nuclear Overhauser effect, Journal of the American Chemical Society 108, 4244-4246.

127. Thanabal, V., De Ropp, J. S., and La Mar, G. N. (1987) Identification of the catalytically important amino acid residue resonances in ferric low-spin horseradish peroxidase with nuclear Overhauser effect measurements, Journal of the American Chemical Society 109, 7516-7525.

128. La Mar, G. N., Hernandez, G., and De Ropp, J. S. (1992) Proton NMR investigation of the influence of interacting sites on the dynamics and thermodynamics of substrate and ligand binding to horseradish peroxidase, Biochemistry 31, 9158-9168.

129. Lee, K.-B., La Mar, G. N., Mansfield, K. E., Smith, K. M., Pochapsky, T. C., and Sligar, S. G. (1993) Interpretation of hyperfine shift patterns in ferricytochromes b5 in terms of angular position of the heme: a sensitive probe for peripheral heme protein interactions, Biochimica et Biophysica Acta (BBA) - Protein Structure and Molecular Enzymology 1202, 189-199.

130. Chen, Z., de Ropp, J. S., Hernandez, G., and La Mar, G. N. (1994) 2D NMR Approaches to Characterizing the Molecular Structure and Dynamic Stability of the Active Site for Cyanide-Inhibited Horseradish Peroxidase, Journal of the American Chemical Society 116, 8772-8783. 
131. de Ropp, J. S., Mandal, P., Brauer, S. L., and La Mar, G. N. (1997) Solution NMR Study of the Electronic and Molecular Structure of the Heme Cavity in High-Spin, Resting State Horseradish Peroxidase, Journal of the American Chemical Society $119,4732-4739$.

132. de Ropp, J. S., Mandal, P. K., and La Mar, G. N. (1998) Solution 1H NMR Investigation of the Heme Cavity and Substrate Binding Site in Cyanide-Inhibited Horseradish Peroxidase $\dagger$, Biochemistry 38, 1077-1086.

133. Chattopadhyay, K., and Mazumdar, S. (1999) Structural and Conformational Stability of Horseradish Peroxidase: Effect of Temperature and $\mathrm{pH}$, Biochemistry 39, 263-270. 


\section{CHAPTER II. ISOLATION AND STRUCTURE DETERMINATION OF A NOVEL HEME IN ALLYLBENZENE-MODIFIED CHLOROPEROXIDASE}

\subsection{Introduction}

Chloroperoxidase (CPO) from the ascomycetous fungus, Caldariomyces fumago (1), is one of the most diverse and versatile catalysts among hemoproteins. CPO is the most extensively studied halogenating enzyme. It is heavy glycosylated ( $\sim 20 \%$ carbohydrates) and has a molecular mass of $42 \mathrm{kDa}$. In addition to halogenation, $\mathrm{CPO}$ can catalyze a variety of oxidations including epoxidation of olefins (2), oxidation of alcohols and aldehydes (3-4), hydroxylation of benzylic and propargylic compounds (5-7), oxidation of amino into nitroso groups (8), $N$-dealkylation (9-10), sulfoxidations (11), as well as classic peroxidase (12) and catalase (13) reactions. Except for the classical heme peroxidase reactions, most catalytic activities of $\mathrm{CPO}$ are also common to cytochromes P450. The x-ray crystal structures of CPO (14) reveals a cysteine thiolate as the proximal ligand to the heme, which is in marked contrast with other heme peroxidases dominated with a proximal histidine ligand.

The catalytic cycle of CPO is initiated by the heterolytic cleavage of a loosely bound $\mathrm{H}_{2} \mathrm{O}_{2}$ molecule, which leads to the formation of a typical heme peroxidase intermediate, compound I. All CPO catalyzed reactions are subsequently facilitated by this highly reactive compound I through direct redox reaction or the formation of other intermediates, such as compound II and the protonated compound II (15).

A unique mechanism-based "suicidal" inactivation of CPO was reported that when incubated with allylbenzene $(\mathrm{AB})$ or other selected terminal unsaturated substrates in the presence of hydrogen peroxide, $\mathrm{CPO}$ will be rapidly and quantitatively converted into a 
green protein species that is devoid of any enzymatic activity (16). Surprisingly, the green $\mathrm{CPO}$ can slowly auto-recover to the native $\mathrm{CPO}$ upon standing with more than $80 \%$ recovery of the original enzyme's activity (Figure 1.6) (17).

Similar mechanism-based heme modifications have also been reported for cytochromes P-450 (18-39), hemoglobin (40), sulfmyoglobin (41), horseradish peroxidase (42), and heme model systems (43-53). Of all known hemoproteins that undergo mechanism-based inactivations, only $\mathrm{CPO}$ can spontaneously regain activity. However, mechanism-based inactivations of a P450 2E1 mutant (34-35) and P450 2B4 as well as its mutant (36) were recently claimed to be partially reversible upon dialysis. The results from the studies of these modified heme systems greatly enhanced the understanding of the active site environment and catalytic mechanism of these hemoproteins.

It is difficult to establish the chemical identity of the modified heme because of the auto-recovery behavior of green CPO. Q-band continuous wave $(\mathrm{CW})$, pulsed electron nuclear double resonance spectroscopy (ENDOR), Mössbauer and electron paramagnetic resonance (EPR) studies of allylbenzene-inactivated CPO had proposed the green heme species as an allylbenzene $\mathrm{N}$-alkylated heme. A nitrogen in one of the heme pyrrole rings is alkylated by the terminal carbon of allylbenzene and the compound I oxygen is ligated with the internal double-bond carbon, forming a five-membered heterocyclic chelate ring $(17,54)$. However, none of the above mentioned techniques was able to establish to which pyrrole nitrogen the allylbenzene moiety is attached.

To better understanding the catalytic mechanism of $\mathrm{CPO}$, the topology of substrate access orientation to the heme active center, the mechanism of spontaneous heme dealkylation and restoration of catalytic activity in this system, I report a novel process 
for establishing the exact structure of the $N$-alkylated heme center of CPO. Being aware of the significantly increased stability of the isolated $N$-alkylated heme (16), the modified heme was extracted out of the green CPO followed by NMR spectroscopic and LC-MS studies to define the structure of the isolated "green heme".

\subsection{Materials and Methods}

\subsubsection{Materials}

Caldariomyces fumago was purchased from ATCC (Manassas, VA). Deuterated solvents were from Cambridge Isotope Laboratories (Andover, MA). Deionized water with $18.2 \mathrm{megohm} \cdot \mathrm{cm}$ resistivity were freshly produced from a Milli-Q BioCel Water Purification System (Millipore, Billerica, MA). All other reagents were of the highest purity available from Fisher Scientific (Pittsburg, PA) or Sigma-Aldrich (St. Louis, MO).

\subsubsection{Growth of Mold and Preparation of CPO}

Stock cultures of C. fumago were inoculated on Potato Dextrose Agar (PDA) plates and grew for 7 days at $28{ }^{\circ} \mathrm{C}$ as a surface mat. The surface mat from three plates of inoculum was used to inoculate $300 \mathrm{~mL}$ of Fructose Peptone Salts (FPS) medium supplemented with $50 \mathrm{mg} / \mathrm{L}$ carbenicillin and homogenized for $30 \mathrm{sec}$ with a sterile tissue homogenizer (Omni TH115). The homogenate was equally divided into six $125 \mathrm{~mL}$ Erlenmeyer flasks plugged with autoclaved cotton balls. After 7 days of growth at $22{ }^{\circ} \mathrm{C}$ in a rotary shaker (Bruker), the mold mycelium was homogenized again and inoculated into six $4 \mathrm{~L}$ baffled culture flasks, each containing $2 \mathrm{~L}$ of FPS medium. The inoculum was grown at $19{ }^{\circ} \mathrm{C}$ in a shaker with a rotation speed of $200 \mathrm{rpm}$. Chloroperoxidase production was monitored kinetically via the standard MCD assay (55) three times a week. The C. fumago medium containing secreted $\mathrm{CPO}$ was harvested when the MCD 
chlorination activity stop increasing, typically 12-14 days after incubation, followed by isolation and purification of $\mathrm{CPO}$ from the growth medium according to the method established by Morris et al. (56) with minor modifications, using acetone instead of ethanol in the solvent fractionation step.

The Reinheitzahl value (Rz, A398 nm/A280 nm) was used to check the purity of the final sample. Protein preparations with Rz values of 1.44 or higher were concentrated to $\sim 3 \mathrm{mM}$ with ultrafiltration and stored at $4{ }^{\circ} \mathrm{C}$ for future use. Concentrations of native CPO were determined using an extinction coefficient of $91200 \mathrm{M}^{-1} \mathrm{~cm}^{-1}$ at $400 \mathrm{~nm}$.

\subsubsection{Preparation, Extraction and Purification of Allylbenzene-Modified Heme}

Green CPO was prepared as described by Debrunner et al. (17). Modified green heme was extracted by the conventional acid-butanone method (57). Since the extracted green heme can automatically form a $\mu$-oxo dimer (54), $0.1 \mu \mathrm{L}$ of $12 \mathrm{~N} \mathrm{HCl}$ was applied to the extracted fraction to depolymerize the dimer. To eliminate the native heme auto-recovered during green heme preparation and other possible contaminant, the extracted green heme was vacuum dried and then subjected to further purification with high-performance liquid chromatography (HPLC) on apHera C18 column (Waters) with a mobile phase of 70:30 $(\mathrm{v} / \mathrm{v})$ methanol/ $\mathrm{H}_{2} \mathrm{O}$. The purified green heme fraction was collected, vacuum dried and then stored at $-20{ }^{\circ} \mathrm{C}$ for structure characterization. Unless otherwise specified, the entire procedure for the green heme preparation was performed at $4{ }^{\circ} \mathrm{C}$.

\subsubsection{Spectroscopic Characterization of Green Heme}

UV-Visible spectrophotometric characterization of the green heme and its cyanide adduct was obtained in methanol at room temperature on a Cary 300 Bio spectrophotometer (Varian, Palo Alto, CA). 
Mass spectra were performed on Finnigan LCQ Deca XP MAX system (ThermoFinnigan, San Jose, CA) with Surveyor autosampler and Surveyor LC Pump. The mass spectrometer was equipped with an ESI source operated in the positive ion mode. Secondary mass spectrometry (MS/MS) experiments were performed to analyze the targeted ions. The operating conditions of the MS detector were optimized with a solution of 90:10 (v/v) acetonitrile/water at a flow rate of $20 \mu \mathrm{L} / \mathrm{min}$. The signal abundance of major peak was maximized as follows: sheath gas flow, 30 arbitrary units; spray voltage, $5 \mathrm{kV}$; heated capillary temperature, $260{ }^{\circ} \mathrm{C}$; capillary voltage, $45 \mathrm{~V}$; tube lens offset, $55 \mathrm{~V}$, a relative collision energy of $30 \%$ was used for all MS/MS experiments with an isolation width of $3.0 \mu$.

The NMR sample was prepared by dissolving green heme in $500 \mu \mathrm{L}$ deuterated methanol-D4. The cyanide adducts of the green heme were prepared by addition of twenty equivalence of cyanide from a freshly made $1 \mathrm{M}$ stock solution of $\mathrm{KCN}$ in $99.9 \%$ $\mathrm{D}_{2} \mathrm{O}$. Demetallation of the green heme was achieved by treatment with $5 \%(\mathrm{v} / \mathrm{v})$ $\mathrm{DCl} /$ methanol-s4 (18).

Proton NMR spectra of both cyanide-bound and demetallated green heme were recorded on a Bruker Avance 600 (Ultrashield) NMR spectrometer operating at a proton frequency of $599.97 \mathrm{MHz}$. The most intense solvent signal was suppressed with presaturation during relaxation delay. Chemical shift values were referenced to the residual HDO signal at $4.76 \mathrm{ppm}$. All NMR spectra were acquired at $298 \mathrm{~K}$ unless otherwise specified.

Phase-sensitive NOESY spectra for both cyanide-bound and demetallated green heme dissolved in DMSO-D6 were acquired with a mixing time of 1.5 to $300 \mathrm{~ms}$ and a 
repetition time of 0.02 to 3 seconds. Typical NOESY spectra were collected with 256 experiments in the F1 dimension using the hypercomplex method (58). Total number of 64-4000 scans were accumulated for each F1 experiment, which was acquired with 2048 complex points in the F2 dimension over a spectral width of 24 to $200 \mathrm{ppm}$. A $200 \mathrm{~ms}$ presaturation with a weak decoupler power was used in all NOESY experiments to suppress the residual solvent signal. Magnitude COSY spectra were acquired with the same parameters in NOESY, using standard Bruker pulse sequence (cosyprqf).

Clean TOCSY (59) spectra of demetallated green heme were recorded over different spectral windows using 2048 F2 points and 256 complex F1 points of 48 scans. Solvent suppression was achieved by a 200-ms direct saturation during the relaxation delay period. A mixing time of $60 \mathrm{~ms}$ was used to allow effective spin lock for protons with different relaxation properties.

All two-dimensional data were processed on a HP Workstation with a Pentium 4 processor using Topspin 1.3 (Bruker). Various apodization functions were employed to emphasize protons with different relaxation properties. For example, apodization over 256, 512, and 1024 points was used to emphasize fast relaxing broad cross-peaks at the expense of resolution, whereas apodization over 2048 points is necessary to emphasize slowly relaxing cross-peaks. All two-dimensional data for the green heme were zerofilled to obtain 2048 x 2048 matrices as required by the large hyperfine shift dispersion exhibited by the paramagnetic nature of this heme adduct.

\subsection{Results}

The modified heme species with a bright green color was isolated from the allylbenzene inactivated CPO. Through purification, a red colored species was separated 
from the dominant green fraction, which should be the protoheme $b$ derived from the trace amount of auto-recovered CPO. Small amount of other green species $(<1 \%)$ was also isolated from the main product, which suggested the presence of isomers of the allylbenzene $N$-alkylated heme. The purified green heme species is very stable at room temperature and shows no noticeable optical change for weeks.

\subsubsection{Mass Analysis}

The Mass spectrum of purified green heme showed a dominant peak at $\mathrm{m} / \mathrm{z} 759.4$ (Figure 2.1A), which can be assigned to a doubly charged $\mu$-oxo dimer as shown in the inset of Figure 2.1A with a total MW of 1517. The secondary MS/MS spectrum of this peak clearly showed the largest fraction at $\mathrm{m} / \mathrm{z} 750.0$ (Figure 2.1B), which is consistent with the MW of allylbenzene $N$-alkylated heme plus an oxygen atom (inset of Figure 2.1B).

Upon addition of 2 equivalents of $\mathrm{HCl}$, the major component of green heme in mass spectrum is observed at $m / z 750.2$, which is consistent with result in the absence of $\mathrm{HCl}$ and previously reported electrospray mass spectrometric analysis of the green $\mathrm{AB}-\mathrm{CPO}(16)$. This $m / z=750.2$ species is therefore assigned to the green heme monomer resulted from the depolymerization of the $\mu$-oxo dimer. The secondary MS/MS spectrum of the $m / z 750.2$ peak (Fig. 2.2) clearly shows the cleavage of one of the propionic acid side chain, removal of the allylbenzene adduct together with the oxygen bridge, and other fragments as shown in the insets of Fig. 2.2. Therefore, our MS studies confirm the presence of a modified heme with an allybenzene moiety added to one of the pyrrole nitrogens as reported in literature. However, the exact position of the modification cannot be established by our MS analysis. To elucidate the chemical nature of allybenzene modified heme, detailed NMR spectroscopic studies were carried out as described in the next section of this chapter. 

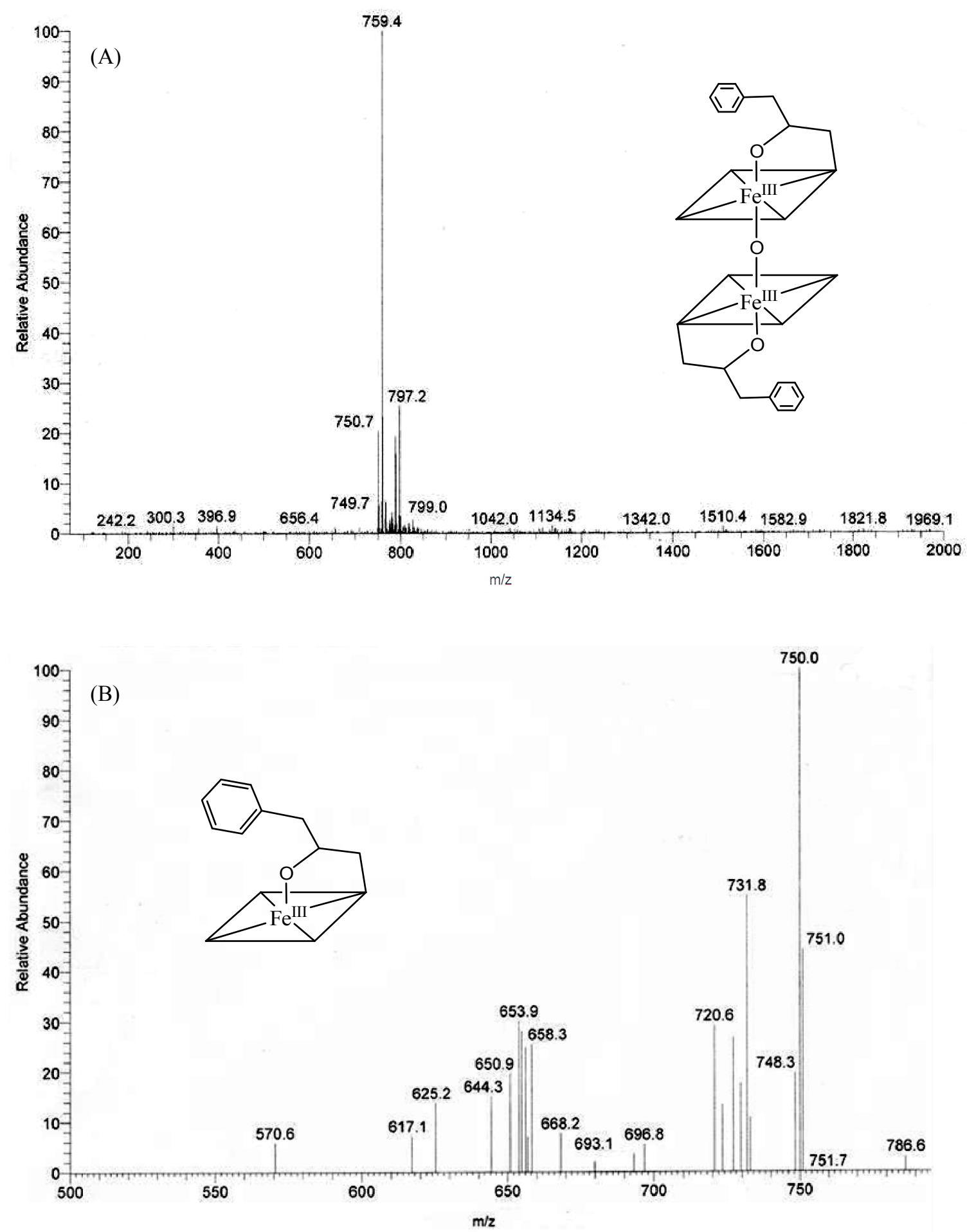

Figure 2.1 MS (A) and MS/MS (B) spectra of purified green species extracted from green CPO. The insets are the schematic structures of allylbenzene modified heme $\mu$-oxo dimer (A) and monomer (B), respectively. 


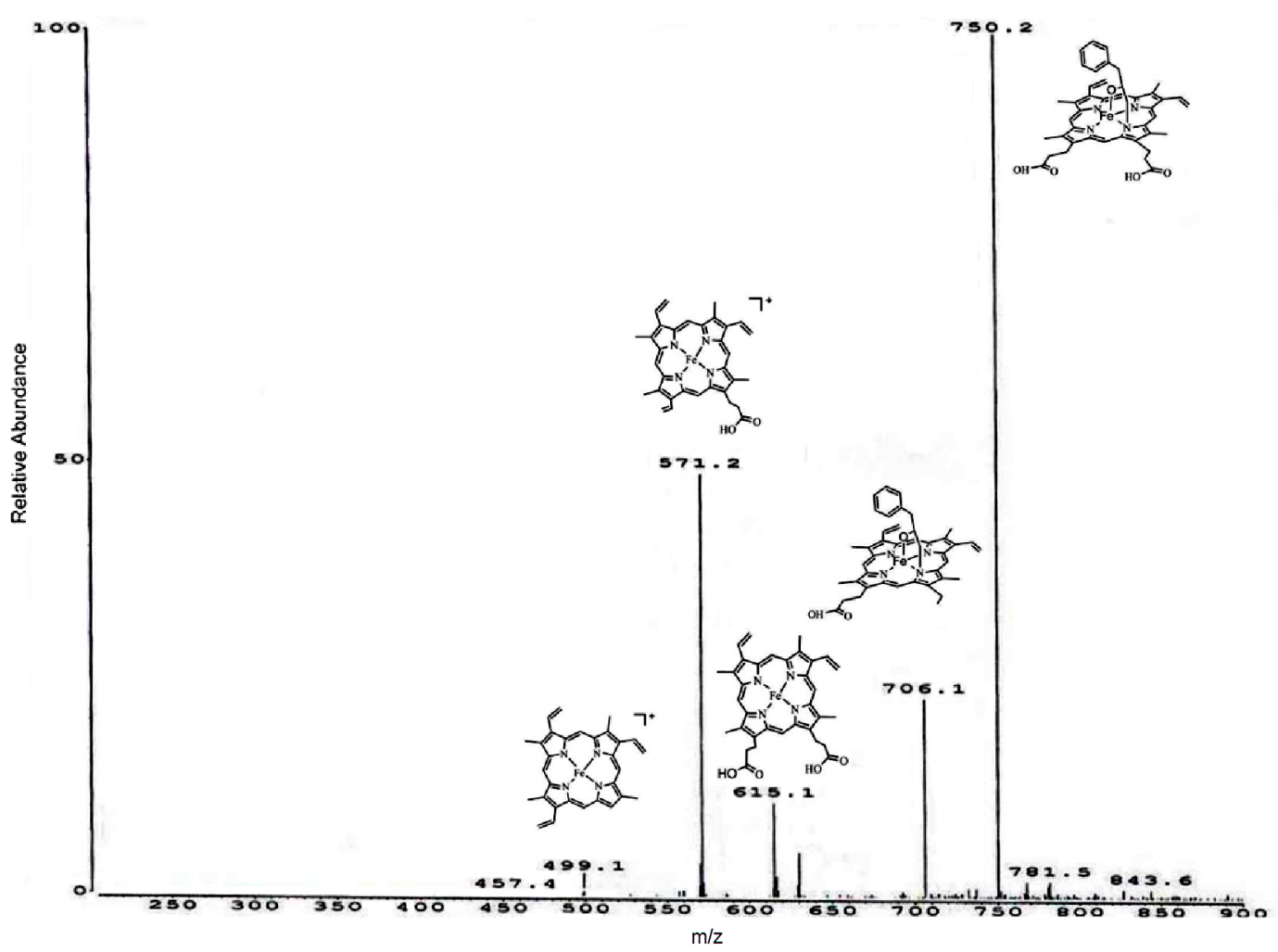

Figure 2.2 MS/MS spectra of depolymerized green heme. The insets are the hypothetic structures of the green heme monomer and subsequent fragments.

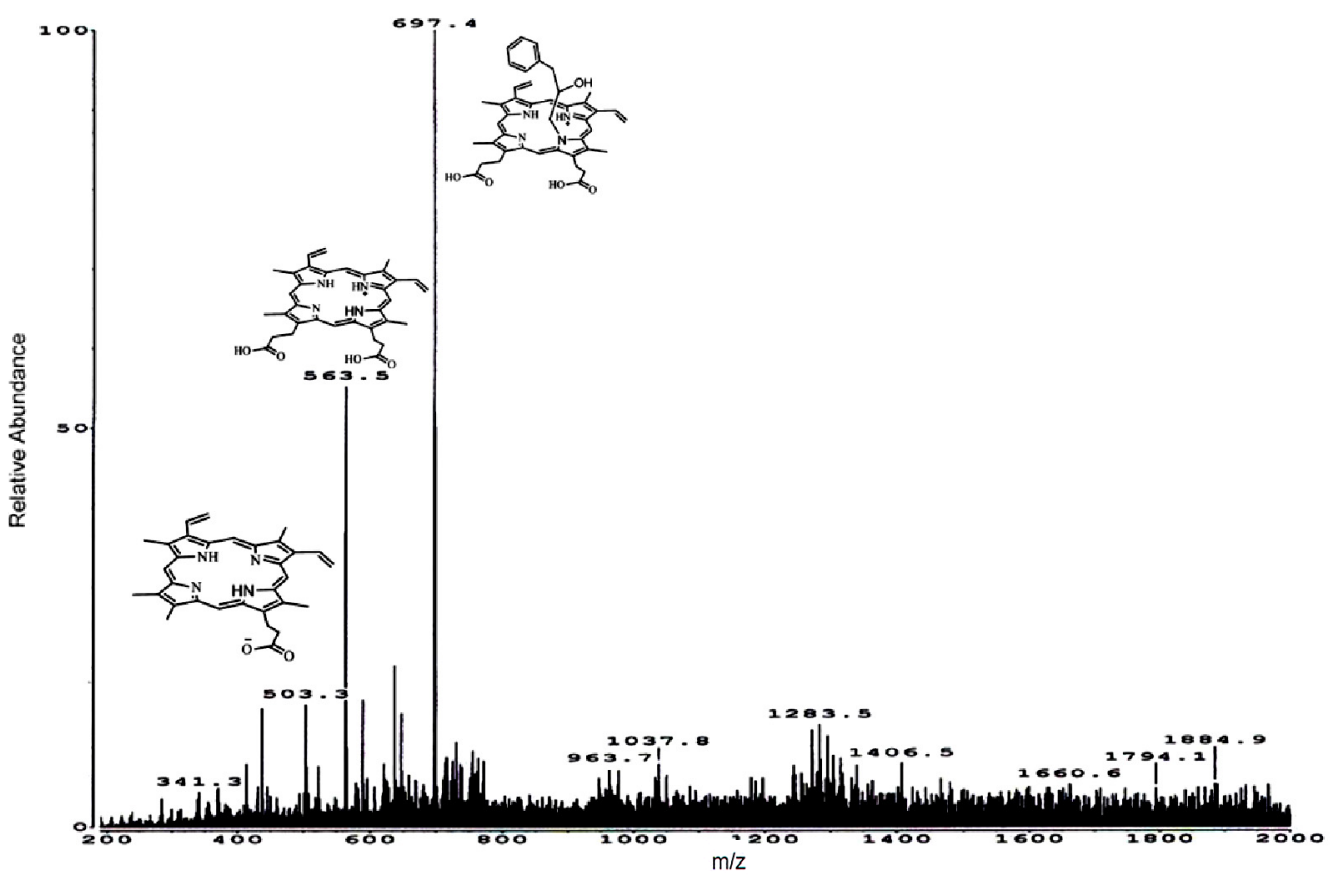

Figure 2.3 MS/MS spectra of demetallated green heme. The insets are the hypothetic structures of the demetallated green heme and subsequent fragments. 
The demetallation of the green heme was achieved by addition of excess amount of $\mathrm{HCl}$ to green heme solution. Mass spectrum of the demetallated green heme confirms the success of iron removal with a major component at $m / z 697.4$ and no peak for the green heme monomer or dimer was observed (Figure 2.3). From the mass spectrum, the demetallated green heme is shown to maintain the allylbenzene $N$-alkylated porphyrin structure with the iron-bound oxygen protonated to form a hydroxyl group.

\subsubsection{UV-Visible Spectroscopy Results}

The UV-visible spectrum of the as-isolated green heme in methanol showed broad electronic absorption bands at 384 and $612 \mathrm{~nm}$, which is inconsistent with previously reported 408, 504, and $596 \mathrm{~nm}$ for $\mu$-oxo green heme (54). The difference may be attributed to solvent effect since the solvent used in the literature was not specified. No observable changes in the UV-vis spectrum of the as-isolated green heme solution was noticed upon addition of $\mathrm{CN}$ suggesting the isolated green heme exists as a $\mu$-oxo dimer. The UV-visible spectrum of the green heme monomer has a Soret band at $404 \mathrm{~nm}$ and blue shifted to $395 \mathrm{~nm}$ upon cyanide binding (Figure 2.4).

\subsubsection{Proton NMR Results}

The proton NMR spectrum of the as-isolated green heme (Figure 2.5A) displays signals within the normal spectral window typical of standard diamagnetic compounds suggesting the presence of a magnetically coupled system, in complete agreement with the $\mu$-oxo dimer nature postulated previously (54). After depolymerization, with addition of concentrated $\mathrm{DCl}$, a typical five-coordinated high-spin iron-porphyrin proton NMR spectrum (Figure 2.5B) is observed. Because of the high-spin nature of the green heme monomer, considerably broad resonances are observed in the NMR spectra that provide 


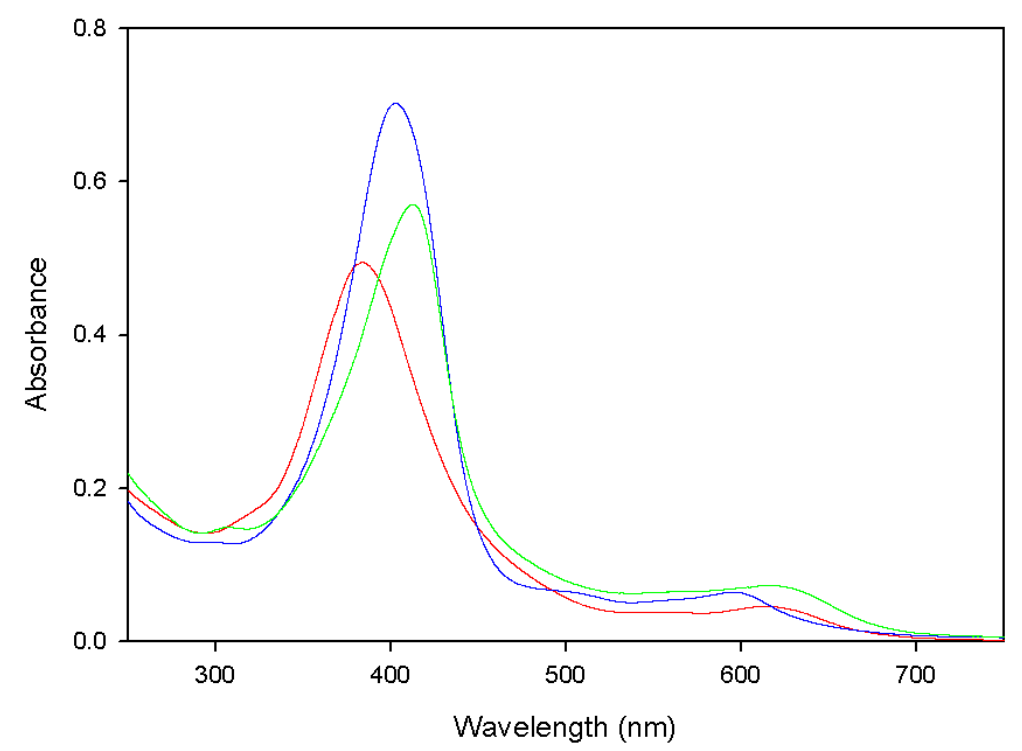

Figure 2.4 UV-vis spectra of the green heme dimer (red), monomer (blue) and cyanide derivative (green) in methanol.

only limited information about the structural properties of the green heme. Therefore, cyanide was added to the sample to convert the green heme monomer to a sixcoordinated low-spin derivative that gives much sharper and better resolved signals in the proton NMR spectrum (Figure 2.5C).

However, although the resonances of green heme- $\mathrm{CN}^{-}$are well resolved in the proton NMR spectrum, no further structural information can be extracted because no Nuclear Overhauser Effect (NOE) can be obtained even with a combination of irradiation power and duration in 1D experiments. Similarly, no cross peaks were detected in all routine 2D experiments including NOESY, COSY, and TOCSY. The lack of NOE information could be resulted from the sizable paramagnetic leakage quenching (60), the molecular mass unfortunately falls into the intermediate motion region, or other unknown reasons. Methods involving changing the temperature, using different ligands, such as azide or imidazole, and using different deuterated solvents, such as chloroform, DMSO and the 
viscous solvent ethylene glycol as suggested by Dr. La Mar $(41,61)$, all failed to solve this frustrating problem. Therefore, the iron had to be removed from the green heme by the addition of an excess amount of DCl to enable further NMR study of the green heme structure. The proton NMR spectrum of the demetallated green heme supports the presence of an $N$-allylbenzene-protoporphyrin adduct (N-ABPP) as shown in Figure 2.5D $(43,62)$.

Complete assignment of the signals for the $N$-ABPP was achieved through twodimensional (2D) NOESY measurements as well as through bond connectivities (COSY and TOCSY) with confirmation through comparison with the assignments made for other $N$-alkylprotoporphyrins (Table 2.1) $(43,62)$. Figure 2.6 shows the NOESY spectrum of $N$ ABPP collected in DMSO-D6 buffer with a mixing time of $35 \mathrm{~ms}$. The COSY and TOCSY spectra as shown in Figure 2.7 and Figure 2.8 clearly shows the correlation between coupled protons and helped us unambiguously confirm the resonance assignments.

\subsection{Discussion}

\subsubsection{Assignment of the Porphyrin Resonances of $N$-ABPP}

The proton NMR spectrum of $N$-ABPP shows a high similarity to the NMR spectra of $\mathrm{N}$-Methylprotoporphyrin IX ( $N$-MePP) isomers and $\mathrm{N}$-griseofulvin-protoporphyrin $(\mathrm{N}$ GfPP) $(43,62)$. The successful assignments of these $N$-alkylprotoporphyrins demonstrated that resonance positions of different proton types in $\mathrm{N}$-alkylporphyrins display the following pattern: (a) meso- $\mathrm{H}$ at 9.9-10.4 ppm, (b) vinyl- $\mathrm{H} \alpha$ at 7.9-8.4 ppm, (c) vinyl- $\mathrm{H} \beta$ at 6.0-6.5 ppm, (d) propionate- $\mathrm{H} \alpha$ at 4.0-4.4 ppm, (e) methyl groups at 3.4$3.8 \mathrm{ppm}$, (f) propionate- $\mathrm{H} \beta$ at approximately $2.9-3.2 \mathrm{ppm}$, and (g) $N$-methyl or methylene protons at -3.0 to $-4.6 \mathrm{ppm}$. Therefore, the four most down-field shifted single-proton resonances located at $10.78,10.72,10.60$ and $10.35 \mathrm{ppm}$, must arise from the four 


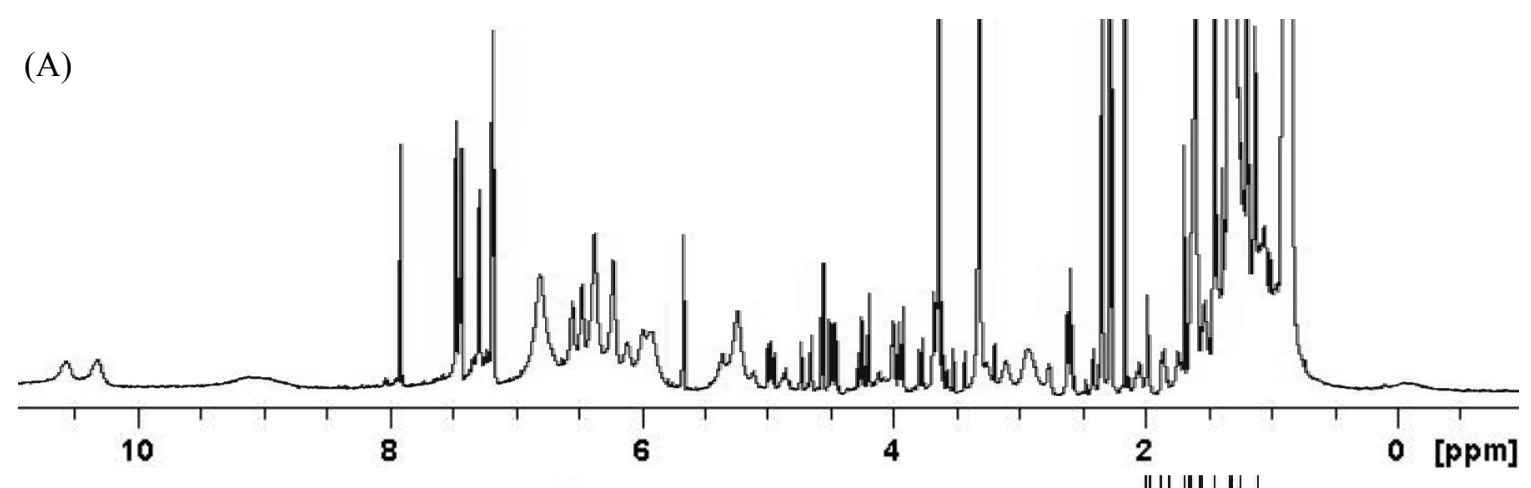

(B)

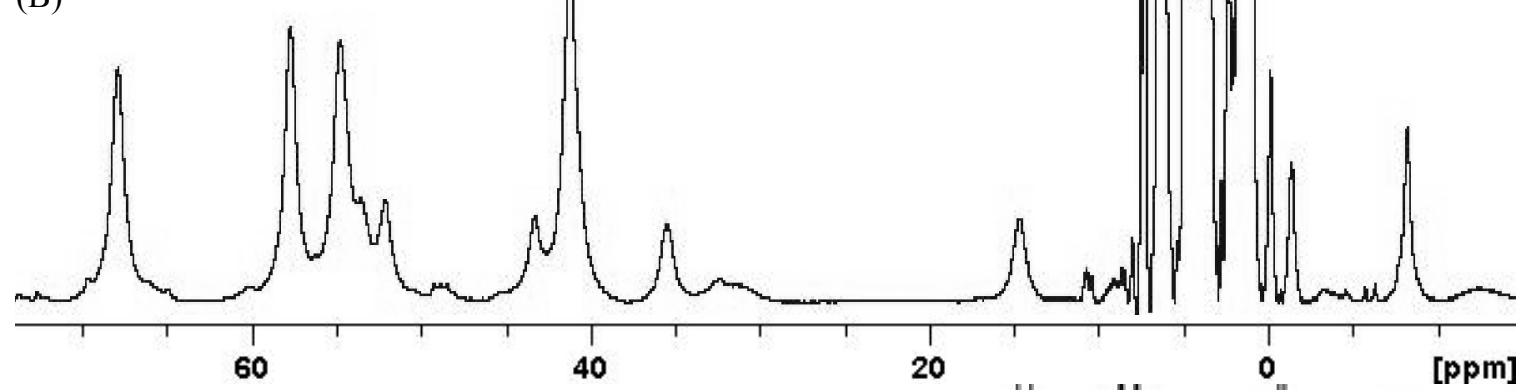

(C)

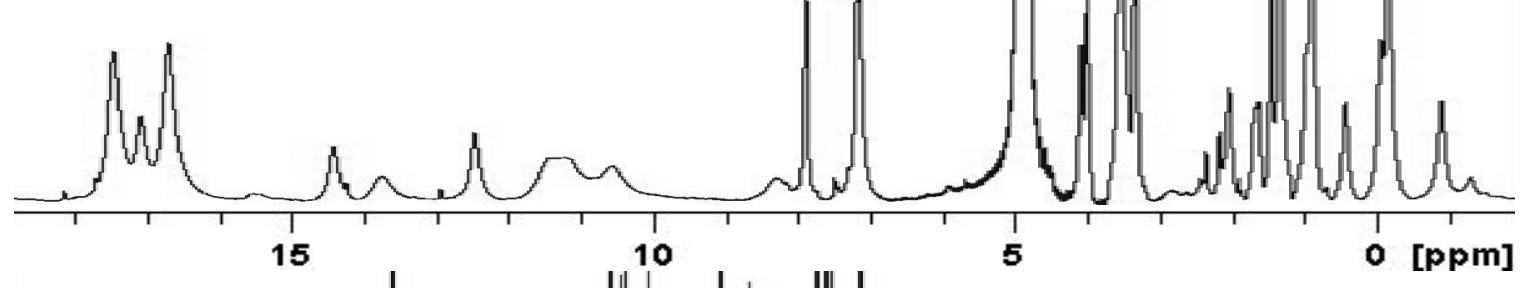

(D)

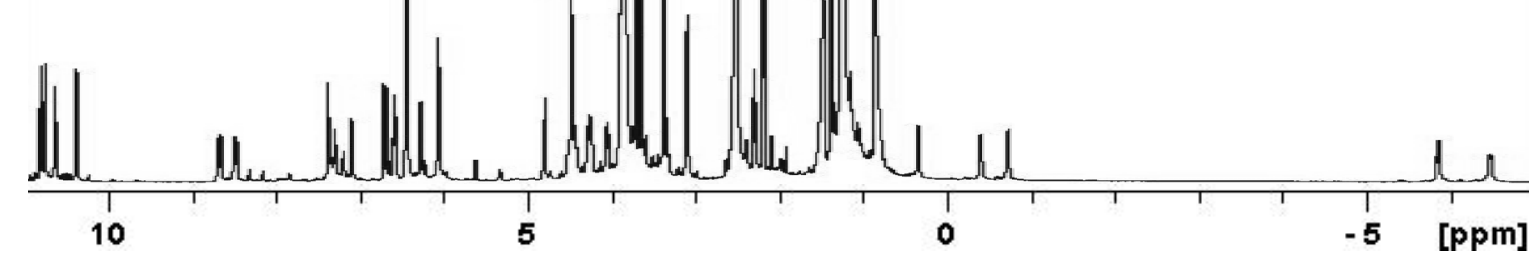

Figure 2.5 The proton NMR spectra of (A) the green heme dimer in deuterated methanol-D4, (B) the green heme monomer in deuterated methanol-D4, (C) the green heme- $\mathrm{CN}^{-}$adduct in deuterated methanol-D4, and (D) the demetallated green heme (N-ABPP) in deuterated DMSO-D6. All spectra were collected at $298 \mathrm{~K}$ with solvent presaturation. 
Table 2.1 Proton NMR chemical shifts (ppm) of the resonances for the $N$-ABPP and the $\mathrm{N}_{\text {III }}$ regioisomer of $N$-methylprotoporphyrin $(N-\mathrm{MePP})(43,62)$.

\begin{tabular}{|c|c|c|c|c|}
\hline \multicolumn{2}{|c|}{ Shift } & Assignment & Shift & Assignment \\
\hline$N$-АВPP & $N$-MePP & Heme & $N$-ABPP & Allylbenzene \\
\hline 10.78 & 9.94 & $\delta$-meso $\mathrm{H}$ & 6.56 & Benzene $p$-H \\
\hline 10.72 & 9.94 & $\gamma$-meso $\mathrm{H}$ & 6.04 & Benzene $m-\mathrm{H}$ \\
\hline 10.60 & 10.10 & $\alpha$-meso $\mathrm{H}$ & 4.46 & Benzene $o-\mathrm{H}$ \\
\hline 10.35 & 10.02 & $\beta$-meso $\mathrm{H}$ & 0.32 & Propyl-1 H \\
\hline 8.65 & 8.34 & 2-H $\alpha$ & -0.42 & Propyl-2 H \\
\hline 8.46 & 8.18 & $4-\mathrm{H} \alpha$ & -0.74 & Propyl-1 H' \\
\hline 6.67 & 6.39 & $2-\mathrm{H} \beta$ & -5.88 & Propyl-3 H \\
\hline 6.42 & 6.23 & $2-\mathrm{H} \beta^{\prime}$ & -6.49 & Propyl-3 H' \\
\hline 6.41 & 6.25 & $4-\mathrm{H} \beta$ & & \\
\hline 6.24 & 6.10 & $4-\mathrm{H} \beta^{\prime}$ & & \\
\hline 4.41 & 4.05 & $6-\mathrm{H} \alpha$ & & \\
\hline 4.26 & 4.05 & $6-\mathrm{H} \alpha^{\prime}$ & & \\
\hline 4.21 & 4.25 & $7-\mathrm{H} \alpha$ & & \\
\hline 4.03 & 4.25 & 7-H $\alpha^{\prime}$ & & \\
\hline 3.89 & 3.75 & 1-CH3 & & \\
\hline 3.66 & 3.58 & $3-\mathrm{CH} 3$ & & \\
\hline 3.61 & 3.47 & $5-\mathrm{CH} 3$ & & \\
\hline 3.36 & 3.49 & 8-CH3 & & \\
\hline 3.08 & 2.76 & $6-\beta \mathrm{CH}_{2}$ & & \\
\hline 2.50 & 3.24 & $7-\beta \mathrm{CH}_{2}$ & & \\
\hline
\end{tabular}

meso protons on the porphyrin ring. The relatively more downfield shifted position of meso protons in $N$-ABPP compared to those in $N$-MePP and $N$-GfPP suggests a bigger ringcurrent effect of the macrocycle due to the out-of-plane distortion of the $\mathrm{N}$-alkylated pyrrole (62-64). Among the four meso protons, the meso-H $\gamma$ and meso-H $\delta$ shall reveal distinctive NOE correlations with only the 6- and 7-propionate protons, and with only two methyl groups (1- and 8- $\mathrm{CH}_{3}$ ), respectively. The meso-H $\alpha$ and meso- $\mathrm{H} \beta$ shall both show NOEs with one methyl and one vinyl-H $\alpha$. In the NOESY spectrum (Figure 2.6), the signal 


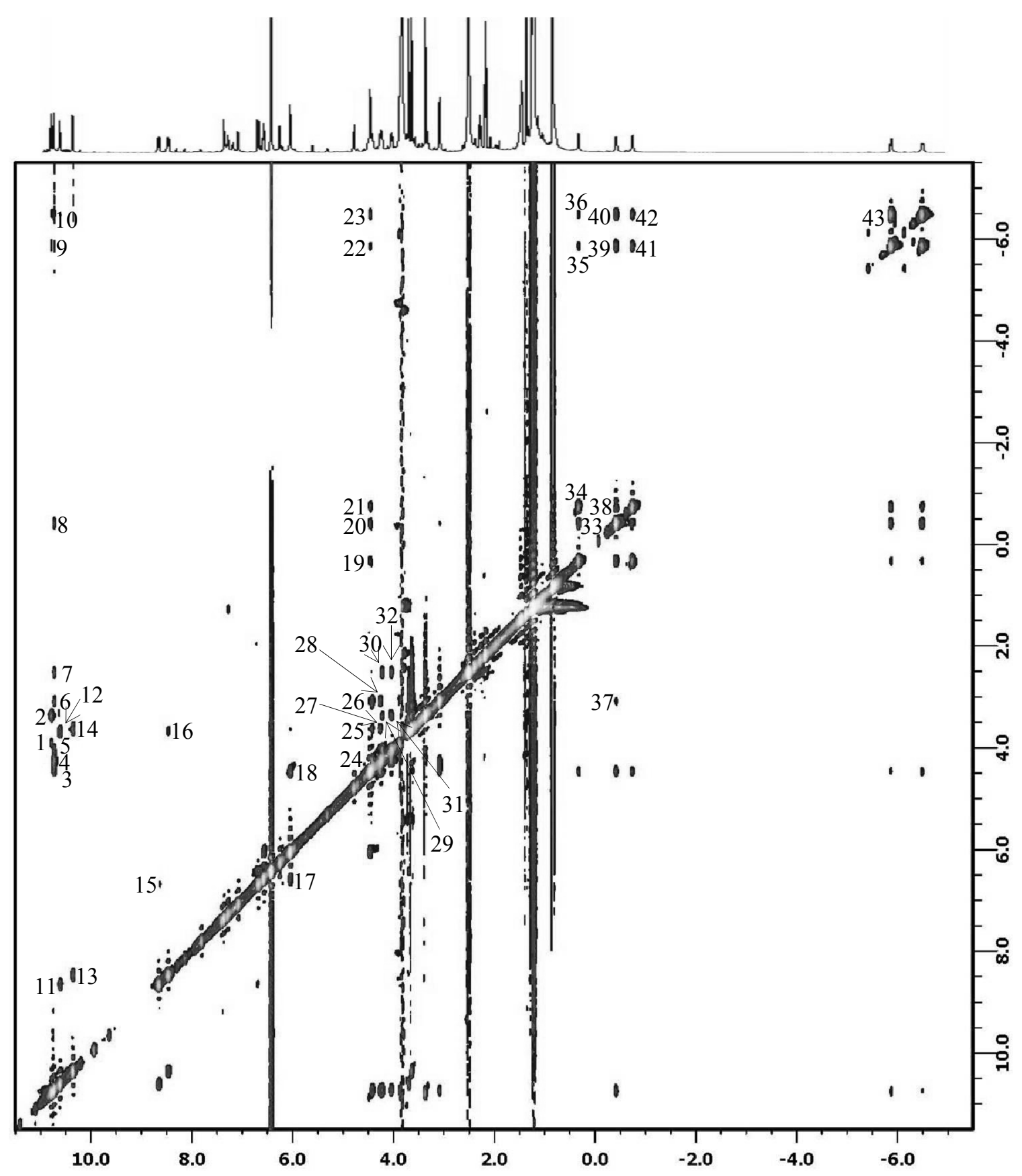

Figure 2.6 The 600-MHz phase-sensitive ${ }^{1} \mathrm{H}$ NOESY spectra of $1 \mathrm{mM} N$-ABPP in DMSO-D6 taken at $298 \mathrm{~K}$ with a mixing time of $35 \mathrm{~ms}$. Cross-peak assignments are as follows: $1, \mathrm{H}-\delta_{\text {meso }}: 1-\mathrm{CH}_{3} ; 2, \mathrm{H}-\delta_{\text {meso }}: 8-\mathrm{CH}_{3} ; 3, \mathrm{H}-$ $\gamma_{\text {meso }}: 6-\mathrm{H} \alpha ; 4, \mathrm{H}-\gamma_{\text {meso }}: 6-\mathrm{H} \alpha^{\prime} / 7-\mathrm{H} \alpha ; 5, \mathrm{H}-\gamma_{\text {meso }}: 7-\mathrm{H} \alpha^{\prime} ; 6, \mathrm{H}-\gamma_{\text {meso }}: 6-\mathrm{H} \beta ; 7, \mathrm{H}-\gamma_{\text {meso }}: 7-\mathrm{H} \beta ; 8, \mathrm{H}-\gamma_{\text {meso }}$ :AB propyl-2H; 9 , $\mathrm{H}-\gamma_{\text {meso }}: \mathrm{AB}$ propyl-3H; $10, \mathrm{H}-\gamma_{\text {meso }}$ :AB propyl-3H'; $11, \mathrm{H}-\alpha_{\text {meso }}: 2-\mathrm{H} \alpha ; 12, \mathrm{H}-\alpha_{\text {meso }}: 3-\mathrm{CH}_{3} ; 13, \mathrm{H}-\beta_{\text {meso }}: 4-\mathrm{H} \alpha ; 14, \mathrm{H}-$ $\beta_{\text {meso }}: 5-\mathrm{CH}_{3} ; 15,2-\mathrm{H} \alpha: 2-\mathrm{H} \beta ; 16,4-\mathrm{H} \alpha: 3-\mathrm{CH}_{3} ; 17, \mathrm{AB}$ benzene $m-\mathrm{H}: \mathrm{AB}$ benzene $p-\mathrm{H} ; 18$, AB benzene $m-\mathrm{H}: \mathrm{AB}$ benzene $o-\mathrm{H} ; 19$, AB benzene $o-\mathrm{H}: \mathrm{AB}$ propyl-1H; 20, AB benzene $o-\mathrm{H}: \mathrm{AB}$ propyl-2H; 21, AB benzene $o-\mathrm{H}: \mathrm{AB}$ propyl-1H'; 22, AB benzene $o$-H:AB propyl-3H; 23, AB benzene $o-\mathrm{H}: A B$ propyl-3H'; 24, 6-H $\alpha: 6-\mathrm{H} \alpha$; $25,6-\mathrm{H} \alpha$ : 5- $\mathrm{CH}_{3} ; 26,6-\mathrm{H} \alpha: 6-\mathrm{H} \beta ; 27,6-\mathrm{H} \alpha$ ': 5- $\mathrm{CH}_{3} ; 28,6-\mathrm{H} \alpha$ ':6-H $\beta ; 29,7-\mathrm{H} \alpha: 8-\mathrm{CH}_{3} ; 30,7-\mathrm{H} \alpha: 7-\mathrm{H} \beta ; 31,7-\mathrm{H} \alpha$ ': 8-CH ; 32, 7-H $\alpha$ '7-H $\beta$; 33, AB propyl-1H:AB propyl-2H; 34, AB propyl-1H:AB propyl-1H'; 35, AB propyl-1H:AB propyl-3H; 36, AB propyl-1H:AB propyl-3H'; 37, AB propyl-2H:6-H $\beta$; 38, AB propyl-2H:AB propyl-1H'; 39, AB propyl-2H:AB propyl-3H; 40, AB propyl-2H:AB propyl-3H'; 41, AB propyl-1H':AB propyl-3H; 42, AB propyl-1H':AB propyl-3H'; 43, AB propyl-3H:AB propyl-3H'. 


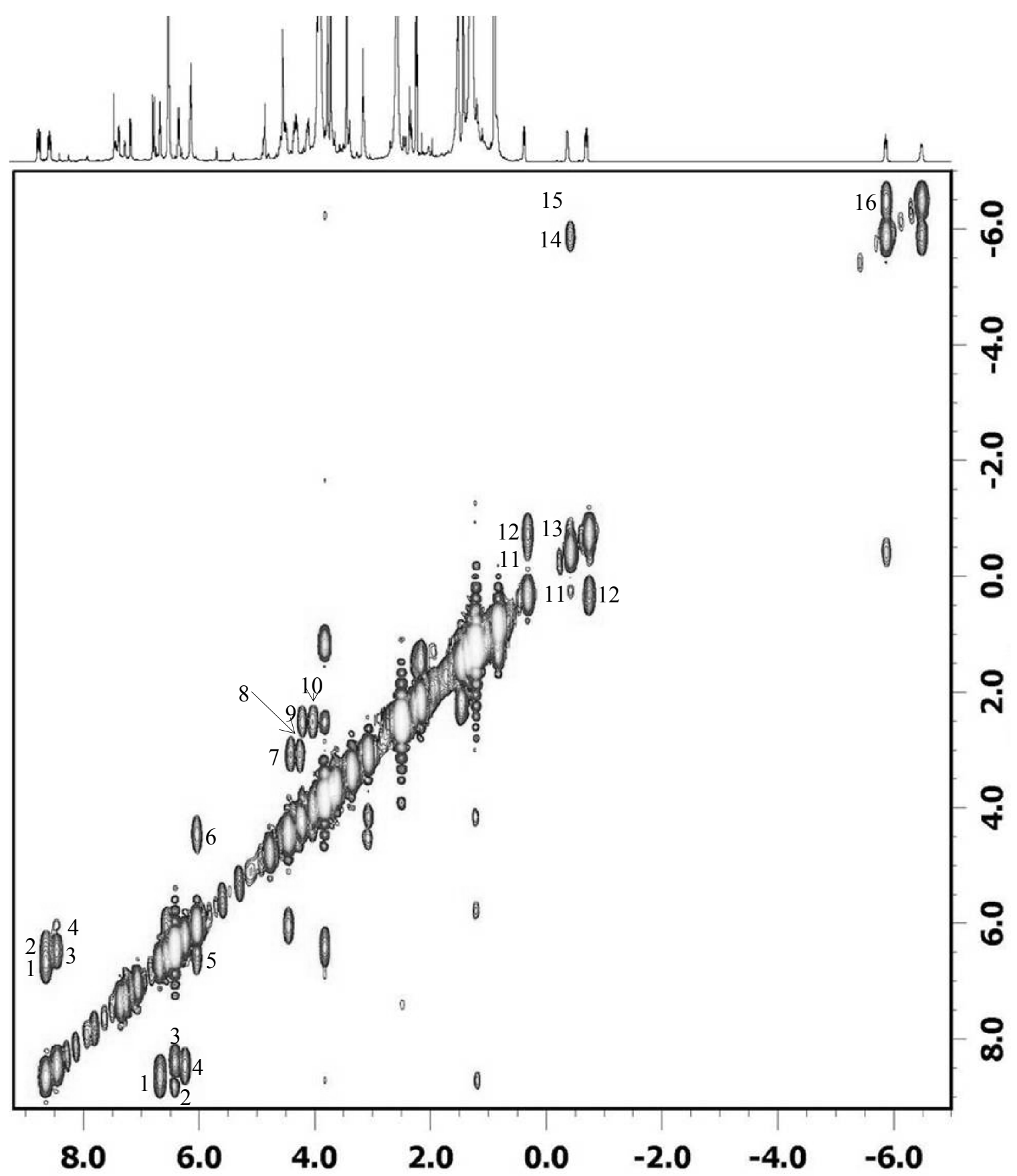

Figure 2.7 The 600-MHz phase-sensitive ${ }^{1} \mathrm{H}$ COSY spectra of $1 \mathrm{mM} \mathrm{N}$-ABPP in DMSO-D6 taken at $298 \mathrm{~K}$. Cross-peak assignments are as follows: 1, 2- $\mathrm{H} \alpha: 2-\mathrm{H} \beta ; 2,2-\mathrm{H} \alpha: 2-\mathrm{H} \beta$ '; 3, 4-H $\alpha: 4-\mathrm{H} \beta ; 4,4-\mathrm{H} \alpha: 4-\mathrm{H} \beta$; ;, $\mathrm{AB}$ benzene $m-\mathrm{H}: \mathrm{AB}$ benzene $p-\mathrm{H} ; 6$, AB benzene $m-\mathrm{H}: \mathrm{AB}$ benzene $o-\mathrm{H} ; 7,6-\mathrm{H} \alpha: 6-\mathrm{H} \beta ; 8,6-\mathrm{H} \alpha$ ' $6-\mathrm{H} \beta ; 9,7-$ $\mathrm{H} \alpha: 7-\mathrm{H} \beta ; 10,7-\mathrm{H} \alpha^{\prime}: 7-\mathrm{H} \beta ; 11, \mathrm{AB}$ propyl-1H:AB propyl-2H; 12, AB propyl-1H:AB propyl-1H'; 13, AB propyl-2H:AB propyl-1H'; 14, AB propyl-2H:AB propyl-3H; 15, AB propyl-2H:AB propyl-3H'; 16, AB propyl-3H:AB propyl-3H'. Cross-peak 15 can only be seen with lower contour levels. 


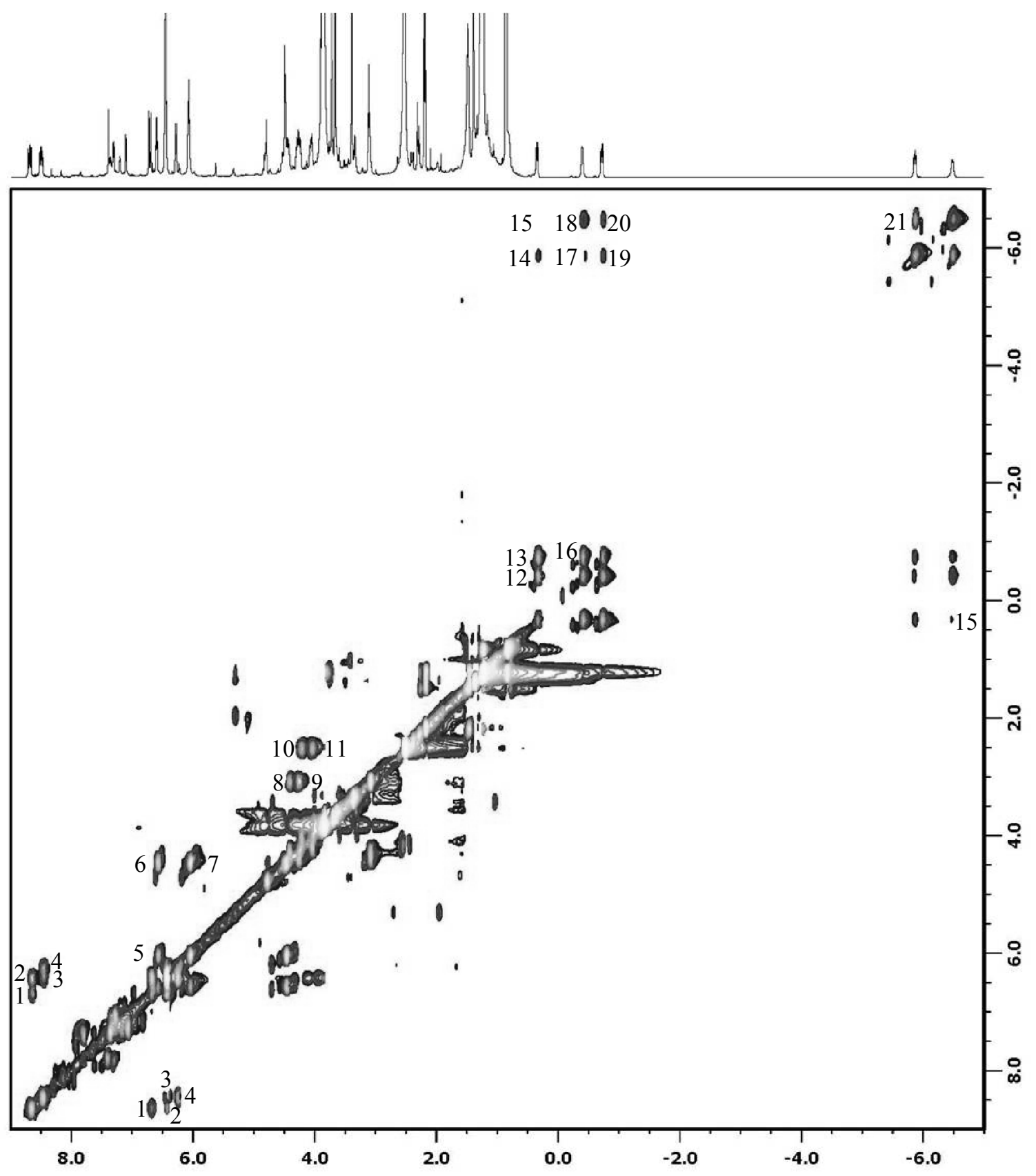

Figure 2.8 The 600-MHz phase-sensitive ${ }^{1} \mathrm{H}$ TOCSY spectra of $1 \mathrm{mM} \mathrm{N}$-ABPP in DMSO-D6 taken at 298 K. Cross-peak assignments are as follows: 1, 2- $\mathrm{H} \alpha: 2-\mathrm{H} \beta ; 2,2-\mathrm{H} \alpha: 2-\mathrm{H} \beta$ '; 3, 4- $\mathrm{H} \alpha: 4-\mathrm{H} \beta ; 4,4-\mathrm{H} \alpha: 4-\mathrm{H} \beta^{\prime} ; 5$, AB benzene $p$-H:AB benzene $m-\mathrm{H} ; 6$, AB benzene $p$-H:AB benzene $o-\mathrm{H} ; 7$, AB benzene $m-\mathrm{H}: \mathrm{AB}$ benzene

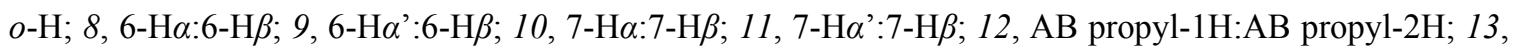
AB propyl-1H:AB propyl-1H'; 14, AB propyl-1H:AB propyl-3H; 15, AB propyl-1H:AB propyl-3H'; 16, AB propyl-2H:AB propyl-1H'; 17, AB propyl-2H:AB propyl-3H; 18, AB propyl-2H:AB propyl-3H'; 19, AB propyl-1H':AB propyl-3H; 20, AB propyl-1H':AB propyl-3H'; 21, AB propyl-3H:AB propyl-3H'. 
at the lowest field $(10.78 \mathrm{ppm})$ shows NOEs to two resonances at 3.89 and $3.36 \mathrm{ppm}$ that correspond to resonance positions of methyl groups. Therefore the $10.78 \mathrm{ppm}$ peak must arise from the meso-H $\delta$ as it is the only proton that could give such an NOE pattern. Consequently, peaks at 3.89 and 3.36 ppm must arise from either 1- or $8-\mathrm{CH}_{3}$. The meso-H at $10.72 \mathrm{ppm}$ displays several NOE correlations with peaks within the propionate $\alpha$-proton and $\beta$-proton regions and can be thus assigned to the meso- $\mathrm{H} \gamma$. The other two meso-Hs at 10.60 and $10.35 \mathrm{ppm}$, so far can be putatively assigned to the meso- $\mathrm{H} \alpha$ and $\mathrm{H} \beta$, respectively, because in other $\mathrm{N}$-alkylprotoporphyrins, meso- $\mathrm{H} \alpha$ is always lower shifted than meso- $\mathrm{H} \beta$. Accordingly, the methyl resonance at $3.66 \mathrm{ppm}$ and the vinyl- $\mathrm{H} \alpha$ resonance at 8.65 ppm both showing NOE to the 10.60 ppm meso-H, can be assigned to the $3-\mathrm{CH}_{3}$ and $2-\mathrm{H} \alpha$, respectively. Similarly, the 10.35 ppm meso-H NOE correlated 3.61 and 8.46 ppm resonances can be assigned to the $5-\mathrm{CH}_{3}$ and $4-\mathrm{H} \alpha$, respectively.

The NOE correlation between the assigned $3-\mathrm{CH}_{3}$ at $3.66 \mathrm{ppm}$ and the $4-\mathrm{H} \alpha$ at 8.46 ppm is in complete agreement with the above proposed assignments. Additionally, the observation of NOE correlations between the $5-\mathrm{CH}_{3}$ at $3.61 \mathrm{ppm}$ and two propionate $\alpha$ protons (4.41 and $4.26 \mathrm{ppm}$ ) also solidly confirmed the previous assignments and leads the assignment of the 4.41 and $4.26 \mathrm{ppm}$ peaks to the 6-propionate $\alpha$-protons. As a result, the other two propionate $\alpha$-protons at 4.21 and $4.03 \mathrm{ppm}$ must derive from the 7propionate group. The assignments of the vinyl $\beta$-protons and propionate $\beta$-protons are archived through COSY and TOCSY by the spin correlations with corresponding vinyl and propionate $\alpha$-protons and are listed in Table 2.1. Therefore, all the 22 nonexchangeable protoporphyrin IX proton resonances are unambiguously assigned through a combination of 2D NMR experiments. 


\subsubsection{Assignment of the Linking Group Resonances of $N$-ABPP}

The spectrum of $N$-ABPP displays two very high-field resonances at -5.88 and -6.49 ppm, which are the result of strong shielding from the ring-current of porphyrin. Similar high-field resonances are assigned to the $N$-linking group protons in $N$-MePP and $N$-GfPP $(43,62)$, and thus the -5.88 and -6.49 ppm peaks must arise from the propyl 3-Hs where the allylbenzene backbone is linked with the heme pyrrole nitrogen. The strong correlations revealed in NOESY, COSY and TOCSY spectra firmly establish the geminal partnerships between these two protons. The inequivalence of the two propyl 3-protons indicates a relatively fixed confirmation of the $N$-ABPP.

Shown in the COSY spectrum (Figure 2.7), the cross-peaks between the $-0.42 \mathrm{ppm}$ and both the propyl 3-protons direct the assignment of the $-0.42 \mathrm{ppm}$ resonance to the propyl 2-H. Subsequently, the 0.32 and -0.74 ppm peaks correlated both with the propyl 2-H and with each other in COSY must arise from the propyl 1-protons.

The two-proton resonance at $4.46 \mathrm{ppm}$ displays NOE correlations to all of the assigned propyl protons and therefore must be derived from the two ortho-Hs of the benzene ring. Again through the COSY and TOCSY spectra, the two meta-Hs and the para- $\mathrm{H}$ on the benzene ring are assigned to the 6.04 and $6.56 \mathrm{ppm}$ peaks, respectively.

The multiplicity of the assigned peaks shown in the 1D spectrum and the complementary correlation information revealed by all the $2 \mathrm{D}$ experiments are in total agreement with the above assignments. Therefore, the unequivocal assignments for all the allylbenzene protons are successfully accomplished. 


\subsubsection{Identification of the Predominant Regioisomer of $\mathrm{N}$-ABPP}

In the NOESY spectrum, the assigned propyl-2H, propyl-3H, and propyl-3H' from allylbenzene moiety, all show indubitable space correlations with the meso-H $\gamma$, which indicates that the $\mathrm{N}$-alkylation must occur at either pyrrole ring III or IV. The conclusive evidence for the $\mathrm{N}$-alkylation position is the NOESY correlation between the propyl-2H of allylbenzene moiety and the 6-propionate $\mathrm{H} \beta$ of the porphyrin side chain, which indicates that the nitrogen of the pyrrole ring III is alkylated by allylbenzene in green CPO.

The exact same conclusion for the identification of the $N$-ABPP regioisomer can also be obtained through analyzing the porphyrin resonances pattern by comparing with the reported results for $N$-MePP regioisomers (62). It is concluded that, in the $\mathrm{N}_{\text {III }}$ and $\mathrm{N}_{\text {IV }}$ regioisomers of $N$-MePP, the propionate $\beta$-proton resonance of the ring that bears the alkyl group is shifted upfield by approximately $0.5 \mathrm{ppm}$, whereas in the $\mathrm{N}_{I}$ and $\mathrm{N}_{\text {II }}$ regioisomers, the $\beta$-protons derived from the two propionate groups are essentially equivalent. For the $N$-ABPP from green $\mathrm{CPO}$, the 6-propionate $\mathrm{H} \beta(2.76 \mathrm{ppm})$ is upfield shifted by 0.48 ppm comparing to the 7-propionate $\mathrm{H} \beta(3.24 \mathrm{ppm})$, indicating the $\mathrm{N}_{\mathrm{III}}{ }^{-}$ alkylation of the allylbenzene modification. Moreover, it is also concluded that in the $\mathrm{N}_{\text {III- }}$ and $\mathrm{N}_{\mathrm{IV}}-\mathrm{MePP}$ regioisomers, the alkylation results in an approximate $0.15 \mathrm{ppm}$ upfield shift for the vinyl $\mathrm{H} \alpha$ resonance on the neighboring ring (4-vinyl for $\mathrm{N}_{\text {III }}$, 2-vinyl for $\mathrm{N}_{\text {IV }}$ ) comparing to that on the ring opposite to the alkylated ring (2-vinyl for $\mathrm{N}_{\text {III }}$, 4vinyl for $\mathrm{N}_{\text {IV }}$ ). While for the $\mathrm{N}_{\mathrm{I}}$ and $\mathrm{N}_{\text {II }}$ regioisomers, the alkylated ring vinyl $\mathrm{H} \alpha$ resonance is approximate $0.4 \mathrm{ppm}$ upfield shifted to the corresponding resonance on the other ring. As shown in Table 2.1, the 4-vinyl $\mathrm{H} \alpha(8.46 \mathrm{ppm})$ of $N$-ABPP is upfield 
shifted by $0.19 \mathrm{ppm}$ compared with the 2-vinyl $\mathrm{H} \alpha(8.65 \mathrm{ppm})$, which therefore also indicates that the $\mathrm{N}$-alkylation is on the nitrogen of pyrrole ring III.

With the above definitive signal assignment, the structure of the $N$-ABPP is clearly defined as $\mathrm{N}_{\mathrm{III}}$-(3-phenyl-2-hydroxyalkyl) protoporphyrin IX (Figure 2.9A) and the green heme extracted from green $\mathrm{CPO}$ is consequently established as shown in Figure 2.9B. By comparing with the $N$-alkylated porphyrin models that has no rigid regiospecificity $(40,46,48,52)$ or only small regiospecificity (29), and the $N$-alkylation of cytochrome P-450 heme that favors the alkylatation of pyrrole ring I $(23,38)$, and IV $(22,38)$, or shows no regiospecificity $(21,24-25,43)$, CPO exhibits a high regio- and stereospecificity with a different $\mathrm{N}$-alkylation position on the prosthetic heme. The unique regioselectivity of the alkylated heme could be related to the specific exposure of the heme group in $\mathrm{CPO}$ and the mechanism of $\mathrm{CPO}$ catalyzed epoxidation in general and allylbenzene initiated inactivation of $\mathrm{CPO}$ in particular as discussed in the next section of this Chapter.
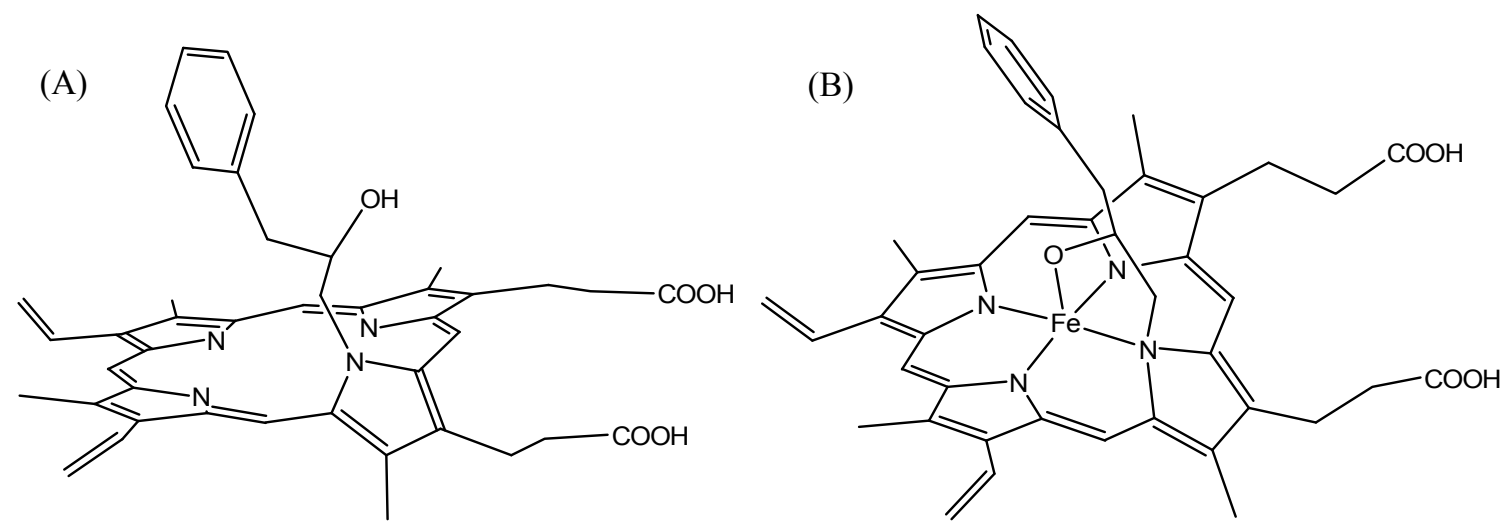

Figure 2.9 The Structure of $N$-ABPP (A) and green heme (B) from CPO calculated by MM2 energy minimization. Note the distance between the AB propyl 2-H and the porphyrin 6-H $\beta$ is consistent with the observed NOE in NOESY. 


\subsubsection{Mechanism of Epoxidation and Substrate Binding of CPO Revealed by the Regiospecific $\mathrm{N}_{\text {III-alkylation }}$}

A well-accepted none-concerted epoxidation versus $\mathrm{N}$-alkylation competition mechanism was proposed for cytochromes P450 as depicted in Figure $2.10(28,30)$. When a terminal olefin substrate encounters the compound I oxygen, the activated oxygen can oxidize either the terminal (pathway A) or the internal (Pathway B) carbon of the double bond. It is postulated that only pathway B can lead to heme $N$-alkylation by the terminal carbon of the substrate, which causes enzyme inactivation (28).

The determination of the high regio- and stereospecific $\mathrm{N}_{\mathrm{III}}$-alkylation of the heme provides a good view point to the $N$-alkylation and active site topology of CPO. Additional constraints rather than electronic factors must exist to control the site of pyrrole $\mathrm{N}$-alkylation at the $\mathrm{CPO}$ active center and the orientation of alkene addition to the heme face. Although currently there is no allylbenzene or styrene bound CPO crystal structure available, useful information can be suggested from other crystal structures of $\mathrm{CPO}$, because the enzyme active site has only minor conformational change upon substrates binding (65). As shown in the crystal structures of CPO (Figure 2.11A) (14, 65), amino acid residues Val67, Ile68, Leu70, Ala71, Asn74, Phe103, Ile179, Val182, Glu183, Phe186 and Ala267 are located at the distal side close to the heme center. These residues together with the heme group, form the substrate binding pocket at the active site.

The regiospecificity of allylbenzene $\mathrm{N}_{\mathrm{III}}$-alkylation strongly suggests that when the internal carbon of the double bond of allylbenzene is oxidized by CPO compound I, the terminal carbon of the double bond is located on top of the pyrrole ring III nitrogen of heme. Therefore, the benzene ring of allylbenzene must head to the opposite side (above 


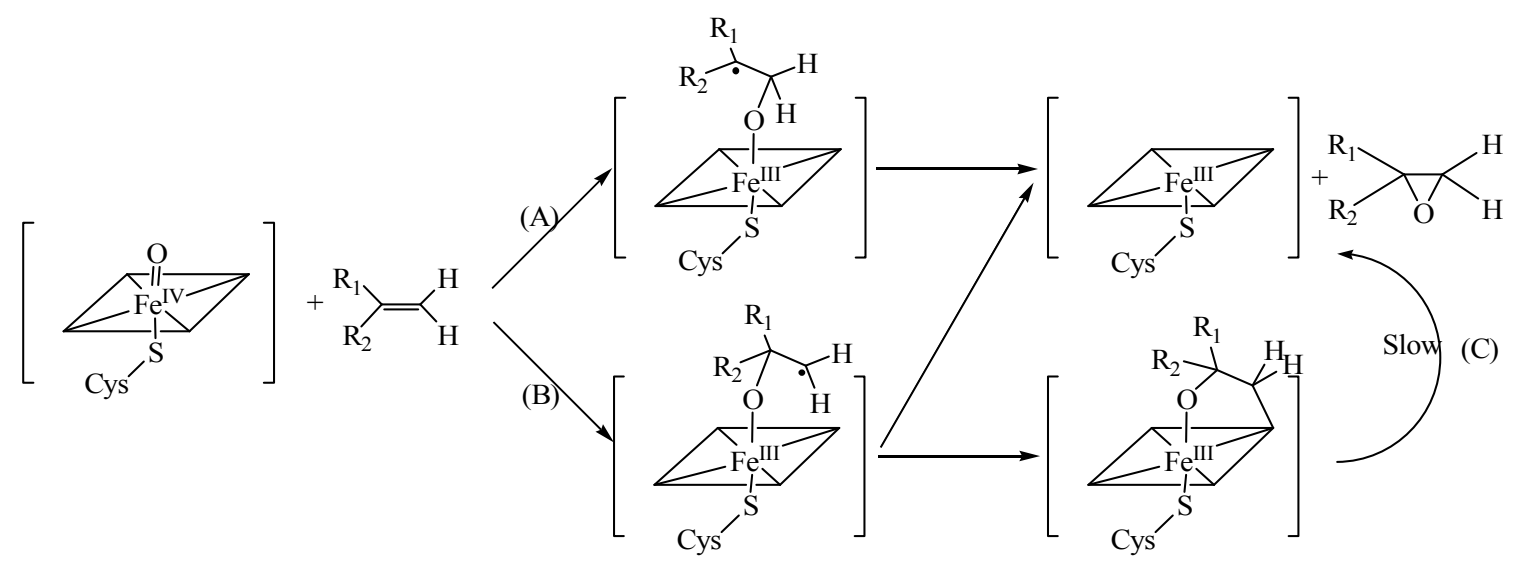

Figure 2.10 Postulated catalytic pathways of (A) epoxidation only or (B) possible for both epoxidation and $\mathrm{N}$-alkylation. The pathway (C) is the recovery of the $\mathrm{N}$-alkylation and is only observed in CPO and two P450 mutants as stated in the introduction.
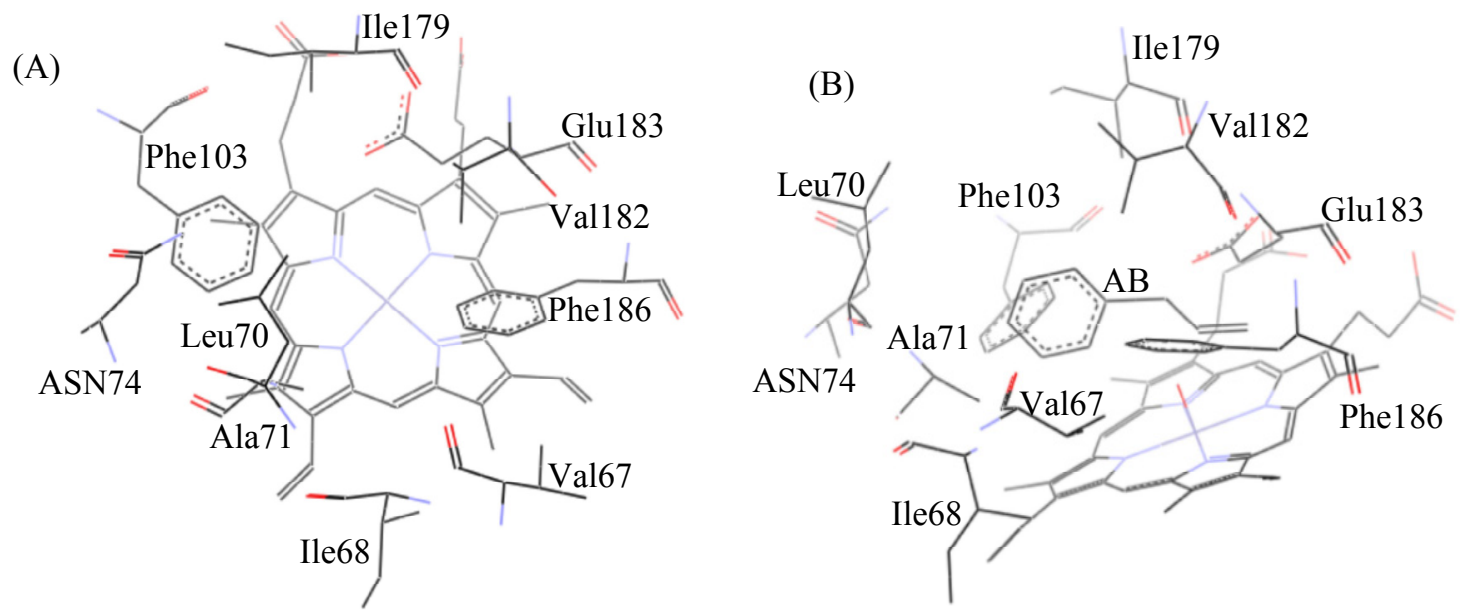

Figure 2.11 CPO distal side binding pocket top view (A), and side view with incorporated substrate allylbenzene (B).

the pyrrole I, Figure 2.11B), which is reasonably the end of the substrate access channel where allylbenzene gets into the active site. Orientations of allylbenzene different from that shown in Figure 2.11B are very unlikely because of the steric constrains caused by the distal residues. Noticeably, the benzene ring of allylbenzene is "coincidentally" sandwiched by the phenyl side chains of Phe103 and Phe186, which are located at the bottom of the substrate access channel and are almost parallel to the heme plane. The 
sandwich structure is also revealed in the crystal structure of CPO complexes with cyclopentanedione, nitrate and acetate (65), indicating that the phenyl side chains of Phe103 and Phe186 may work together as a clamp to hold substrates at fixed orientation, leading to the formation of one enantiomer. The benzene ring of allylbenzene is therefore oriented perpendicular to the heme plane when allylbenzene enters the active site, placing the double bond carbons on top of pyrrole III that facillitates the $\mathrm{N}$-alkylation pathway and affords the $\mathrm{N}_{\mathrm{III}}$-alkylated product. The flexibility of Phe103 (14) makes the doublephenyl clamp capable of holding substrates using steric and edge-to-face $\pi-\pi$ interactions. Meanwhile, Asp74 and Glu183, with their hydrophilic side chains, may also help to guide allylbenzene. Finally, other distal side residues could provide steric hindrances to further control substrate orientation (Figure 2.11B).

Similar assumption was made concerning the function of Phe103 and Phe186 in the oxidation of benzyl alcohol catalyzed by CPO (3), although no direct or indirect evidence was provided. Therefore, it is the first time that the Phe103-Phe186 double phenyl clamp function is demonstrated with experimental support. To the best of my knowledge, this double phenyl clamp motif has never been reported in literature. Although more evidence is required to corroborate this novel motif, it extends and inspires our understanding of enzyme substrate binding strategy.

The proposed CPO active site topology together with the above mentioned epoxidation versus $\mathrm{N}$-alkylation mechanism can flawlessly explain the spontaneous restoration of the green $\mathrm{CPO}$ and the enantioselectivity of $\mathrm{CPO}$ catalyzed epoxidations. With the double-phenyl clamp holding the benzene ring of allylbenzene, a small distortion force from the steric clashes with distal residues is applied to the $\mathrm{N}$-alkylation 
bond, which will decrease its stability and lead to the epoxidation reaction. This is why the extracted green heme, without the interference of the protein backbone, becomes much more stable in either dimer or monomer forms. For CPO catalyzed enantioselective epoxidations, using the styrene epoxidation as an example, the double-phenyl clamp holds the benzene ring of styrene at approximately the same position as that of allylbenzene. The terminal carbon of the double bond will be placed close to the Compound I oxygen because it is one carbon shorter than styrene, and therefore undertakes the epoxidation pathway, which strongly supports the proposed mechanism shown in Figure 2.10. The highly enantioselective epoxidation activity can be attributed to the presence of the double-phenyl clamp that holds the benzene ring at a orientation that favors the formation of $(\mathrm{R})$-enantiomer. This hypothesis is supported by the fact that (R)-styrene epoxide $(\sim 50 \%$ ee) is the main product from $\mathrm{CPO}$ catalyzed oxidation of styrene (66).

The double-phenyl clamp theory is strongly supported by the CPO F103A mutant (Wang, et al. unpublished results), in which the phenyl side chain of Phe103 is removed and the double-phenyl clamp function is disabled. This active site change shall not stop the $N$-alkylation of heme, but may affect the stability of the $\mathrm{N}$-alkylated heme product because the deletion of the phenyl side chain can remove or reduce the distortion force. As predicted, this mutant can be inactivated by allylbenzene but is not able to reverse to the resting state upon standing. Additionally, the activity and enantioselectivity of the epoxidation will also be affected by this mutation. For small substrates such as styrene, the ee value of the product should be decreased because the removal of the phenyl ring will allow the substrate rotate more freely. Indeed, the styrene epoxidation product of 
CPO F103A mutant only has an ee of $\sim 3 \%$ (Chen, et al. unpublished results), a dramatic decrease compared to that of wide-type CPO.

In conclusion, the modified heme in allylbenzene inactivated CPO was extracted, demetallated and characterized by UV-vis, ES-MS/MS, and extensive 1- and 2-D NMR methods. The results indisputably demonstrated that allylbenzene induced alkylation of heme occurs at the nitrogen atom of heme pyrrole ring III in CPO. Based on this discovery, a new mechanism of CPO catalysis and topology of heme active site is proposed, in which a Phe103-Phe186 double-phenyl clamp together with other distal residues are suggested to play an important role in adjusting the position of phenyl substrates, making CPO a highly stereospecific catalyst. This mechanism could be further explored by determining the crystal structure of $\mathrm{CPO}$ complexed with allylbenzene or styrene, site-specific mutagenesis, specially designed substrate and theoretical calculations, and could be expanded in exploring the inhibition and epoxidation of P450 enzymes.

\subsection{References}

1. Shaw, P. D., and Hager, L. P. (1959) An Enzymatic Chlorination Reaction, Journal of the American Chemical Society 81, 1011-1012.

2. Lakner, F. J., and Hager, L. P. (1996) Chloroperoxidase as Enantioselective Epoxidation Catalyst: An Efficient Synthesis of (R)-(-)-Mevalonolactone, The Journal of Organic Chemistry 61, 3923-3925.

3. Baciocchi, E., Manduchi, L., and Lanzalunga, O. (1999) Prochiral selectivity and deuterium kinetic isotope effect in the oxidation of benzyl alcohol catalyzed by chloroperoxidase, Chemical Communications, 1715-1716.

4. Zaks, A., and Dodds, D. R. (1995) Chloroperoxidase-catalyzed asymmetric oxidations: substrate specificity and mechanistic study, Journal of the American Chemical Society 117, 10419-10424. 
5. Miller, V. P., Tschirretguth, R. A., and Demontellano, P. R. O. (1995) Chloroperoxidase-Catalyzed Benzylic Hydroxylation, Archives of Biochemistry and Biophysics 319, 333-340.

6. Hu, S., and Hager, L. P. (1998) Unusual Propargylic Oxidations Catalyzed by Chloroperoxidase, Biochemical and Biophysical Research Communications 253, 544-546.

7. Hu, S., and Hager, L. P. (1999) Highly Enantioselective Propargylic Hydroxylations Catalyzed by Chloroperoxidase, Journal of the American Chemical Society 121, 872-873.

8. Corbett, M., Chipko, B., and Baden, D. (1978) Chloroperoxidase-catalysed oxidation of 4-chloroaniline to 4-chloronitrosobenze, Biochemical Journal 175, 353-360.

9. Kedderis, G. L., Koop, D. R., and Hollenberg, P. F. (1980) N-Demethylation reactions catalyzed by chloroperoxidase, Journal of Biological Chemistry 255, 10174-10182.

10. Okazaki, O., and Guengerich, F. P. (1993) Evidence for specific base catalysis in N-dealkylation reactions catalyzed by cytochrome P450 and chloroperoxidase. Differences in rates of deprotonation of aminium radicals as an explanation for high kinetic hydrogen isotope effects observed with peroxidases, Journal of Biological Chemistry 268, 1546-1552.

11. Colonna, S., Gaggero, N., Manfredi, A., Casella, L., Gullotti, M., Carrea, G., and Pasta, P. (1990) Enantioselective oxidations of sulfides catalyzed by chloroperoxidase, Biochemistry 29, 10465-10468.

12. La Rotta H, C., and Bon, E. (2002) 4-Chlorophenol degradation by chloroperoxidase from Caldariomyces fumago: formation of insoluble products, Applied Biochemistry and Biotechnology 98, 191-203.

13. Dunford, H. B., Lambeir, A.-M., Kashem, M. A., and Pickard, M. (1987) On the mechanism of chlorination by chloroperoxidase, Archives of Biochemistry and Biophysics 252, 292-302.

14. Sundaramoorthy, M., Terner, J., and Poulos, T. L. (1995) The crystal structure of chloroperoxidase: a heme peroxidase-cytochrome $\mathrm{P} 450$ functional hybrid, Structure 3, 1367-1378.

15. Hofrichter, M., and Ullrich, R. (2006) Heme-thiolate haloperoxidases: versatile biocatalysts with biotechnological and environmental significance, Applied Microbiology and Biotechnology 71, 276-288. 
16. Dexter, A. F., and Hager, L. P. (1995) Transient Heme N-Alkylation of Chloroperoxidase by Terminal Alkenes and Alkynes, Journal of the American Chemical Society 117, 817-818.

17. Debrunner, P., Dexter, A., Schulz, C., Xia, Y.-M., and Hager, L. P. (1996) Mossbauer and electron paramagnetic resonance studies of chloroperoxidase following mechanism-based inactivation with allylbenzene, Proceedings of the National Academy of Sciences 93, 12791-12798.

18. Ortiz de Montellano, P. R., and Kunze, K. L. (1980) Self-catalyzed inactivation of hepatic cytochrome P-450 by ethynyl substrates, Journal of Biological Chemistry $255,5578-5585$.

19. Ortiz de Montellano, P. R., Kunze, K. L., and Mico, B. A. (1980) Destruction of Cytochrome P-450 by Olefins: N-Alkylation of Prosthetic Heme Molecular Pharmacology 18, 602-605.

20. Ortiz de Montellano, P. R., and Mico, B. A. (1980) Destruction of Cytochrome P450 by Ethylene and Other Olefins, Molecular Pharmacology 18, 128-135.

21. Ortiz de Montellano, P. R., Beilan, H. S., and Kunze, K. L. (1981) NAlkylprotoporphyrin IX formation in 3,5-dicarbethoxy-1,4-dihydrocollidinetreated rats. Transfer of the alkyl group from the substrate to the porphyrin, Journal of Biological Chemistry 256, 6708-6713.

22. Ortiz de Montellano, P. R., Beilan, H. S., Kunze, K. L., and Mico, B. A. (1981) Destruction of cytochrome P-450 by ethylene. Structure of the resulting prosthetic heme adduct, Journal of Biological Chemistry 256, 4395-4399.

23. Ortiz de Montellano, P. R., and Kunze, K. L. (1981) Cytochrome P-450 inactivation: structure of the prosthetic heme adduct with propyne, Biochemistry 20, 7266-7271.

24. Ortiz de Montellano, P. R., Beilan, H. S., and Mathews, J. M. (1982) Alkylation of the prosthetic heme in cytochrome P-450 during oxidative metabolism of the sedative-hypnotic ethchlorvynol, Journal of Medicinal Chemistry 25, 1174-1179.

25. Ortiz de Montellano, P. R., Kunze, K. L., Beilan, H. S., and Wheeler, C. (1982) Destruction of cytochrome P-450 by vinyl fluoride, fluroxene, and acetylene. Evidence for a radical intermediate in olefin oxidation, Biochemistry 21, 13311339.

26. Kunze, K. L., Mangold, B. L. K., Beilan, C. H., and Ortiz de Montellano, P. R. (1983) The cytochrome P-450 active site. Regiospecificity of prosthetic heme alkylation by olefins and acetylenes., Journal of Biological Chemistry 258, 42024207. 
27. Ortiz de Montellano, P. R., Wheeler, C., Kunze, K. L., and Reich, N. (1983) Stereochemistry of cytochrome P-450-catalyzed epoxidation and prosthetic heme alkylation, Journal of Biological Chemistry 258, 4208-4213.

28. Luke, B. T., Collins, J. R., Loew, G. H., and McLean, A. D. (1990) Theoretical investigations of terminal alkenes as putative suicide substrates of cytochrome P450, Journal of the American Chemical Society 112, 8686-8691.

29. Shirane, N., Sui, Z., Peterson, J. A., and Ortiz de Montellano, P. R. (1993) Cytochrome P450BM-3 (CYP102): Regiospecificity of oxidation of omegaunsaturated fatty acids and mechanism-based inactivation, Biochemistry 32, $13732-13741$.

30. De Visser, S. P., Ogliaro, F., Harris, N., and Shaik, S. (2001) Multi-State Epoxidation of Ethene by Cytochrome P450: A Quantum Chemical Study, Journal of the American Chemical Society 123, 3037-3047.

31. Henne, K. R., Kunze, K. L., Zheng, Y.-M., Christmas, P., Soberman, R. J., and Rettie, A. E. (2001) Covalent Linkage of Prosthetic Heme to CYP4 Family P450 Enzymes, Biochemistry 40, 12925-12931.

32. LeBrun, L. A., Xu, F., Kroetz, D. L., and Ortiz de Montellano, P. R. (2002) Covalent Attachment of the Heme Prosthetic Group in the CYP4F Cytochrome P450 Family, Biochemistry 41, 5931-5937.

33. Shaik, S., de Visser, S. P., Ogliaro, F., Schwarz, H., and Schröder, D. (2002) Two-state reactivity mechanisms of hydroxylation and epoxidation by cytochrome P-450 revealed by theory, Current Opinion in Chemical Biology 6, $556-567$.

34. Blobaum, A. L., Kent, U. M., Alworth, W. L., and Hollenberg, P. F. (2004) Novel Reversible Inactivation of Cytochrome P450 2E1 T303A by tert-Butyl Acetylene: The Role of Threonine 303 in Proton Delivery to the Active Site of Cytochrome P450 2E1, Journal of Pharmacology and Experimental Therapeutics 310, 281290.

35. Lu, Y., Kent, U. M., Wang, S., and Hollenberg, P. F. (2004) Formation of a novel reversible cytochrome P450 spectral intermediate: role of threonine 303 in P450 2E1 inactivation, Biochemistry 43, 11942-11952.

36. Harris, D. L., and Hollenberg, P. F. (2005) P450 Active Site Architecture and Reversibility: Inactivation of Cytochromes P450 2B4 and 2B4 T302A by tertButyl Acetylenes?, Biochemistry 44, 3831-3844. 
37. Blobaum, A. L. (2006) Mechanism-Based Inactivation and Reversibility: Is Therer a New Trend in the Inactivation of Cytochrome P450 Enzymes?, Drug Metabolism and Disposition 34, 1-7.

38. Kunze, K. L., Wheeler, C., Beilan, H. S., and Ortiz de Montellano, P. R. (1983) The cytochrome P-450 active site. Regiospecificity of prosthetic heme alkylation by olefins and acetylenes., Journal of Biological Chemistry 258, 4202-4207.

39. Ortiz de Montellano, P. R., Wheeler, C., Kunze, K. L., and Reich, N. O. (1983) Stereochemistry of cytochrome P-450-catalyzed epoxidation and prosthetic heme alkylation, Journal of Biological Chemistry 258, 4208-4213.

40. Ortiz de Montellano, P. R., and Kunze, K. L. (1981) Formation of N-phenylheme in the hemolytic reaction of phenylhydrazine with hemoglobin, Journal of the American Chemical Society 103, 6534-6536.

41. Chatfield, M. J., La Mar, G. N., Parker, W. O., Smith, K. M., Leung, H. K., and Morris, I. K. (1988) Nuclear magnetic resonance study of the molecular and electronic structure of the stable green sulfhemin prosthetic groups extracted from sulfmyoglobin, Journal of the American Chemical Society 110, 6352-6358.

42. Huang, L., Wojciechowski, G., and Ortiz de Montellano, P. R. (2005) Prosthetic Heme Modification during Halide Ion Oxidation. Demonstration of Chloride Oxidation by Horseradish Peroxidase, Journal of the American Chemical Society $127,5345-5353$.

43. Kunze, K. L., and Ortiz de Montellano, P. R. (1981) N-Methylprotoporphyrin IX. Identification by NMR of the nitrogen alkylated in each of the four isomers, Journal of the American Chemical Society 103, 4225-4230.

44. Mashiko, T., Dolphin, D., Nakano, T., and Traylor, T. G. (1985) NAlkylporphyrin formation during the reactions of cytochrome P-450 model systems, Journal of the American Chemical Society 107, 3735-3736.

45. Collman, J. P., Hampton, P. D., and Brauman, J. I. (1986) Stereochemical and mechanistic studies of the "suicide" event in biomimetic P-450 olefin epoxidation, Journal of the American Chemical Society 108, 7861-7862.

46. Artaud, I., Devocelle, L., Battioni, J. P., Girault, J. P., and Mansuy, D. (1987) Suicidal inactivation of iron-porphyrin catalysts during 1-alkene oxidation. Isolation of a new type of N-alkylporphyrin, Journal of the American Chemical Society 109, 3782-3783.

47. Lavallee, D. K. (1987) The Chemistry and Biochemistry of N-Substituted Porphyrins, VCH, New York. 
48. Traylor, T. G., Nakano, T., Miksztal, A. R., and Dunlap, B. E. (1987) Transient formation of $\mathrm{N}$-alkylhemins during hemin-catalyzed epoxidation of norbornene. Evidence concerning the mechanism of epoxidation, Journal of the American Chemical Society 109, 3625-3632.

49. Balch, A. L., Cornman, C. R., Latos-Grazynski, L., and Olmstead, M. M. (1990) Characterization of five- and six-coordinate iron(III) complexes of Nmethylporphyrins, Journal of the American Chemical Society 112, 7552-7558.

50. Collman, J. P., Hampton, P. D., and Brauman, J. I. (1990) Suicide inactivation of cytochrome P-450 model compounds by terminal olefins. 2. Steric and electronic effects in heme N-alkylation and epoxidation, Journal of the American Chemical Society 112, 2986-2998.

51. Collman, J. P., Hampton, P. D., and Brauman, J. T. (1990) Suicide inactivation of cytochrome P-450 model compounds by terminal olefins. 1. A mechanistic study of heme N-alkylation and epoxidation, Journal of the American Chemical Society 112, 2977-2986.

52. Tian, Z.-Q., Richards, J. L., and Traylor, T. G. (1995) Formation of Both Primary and Secondary N-Alkylhemins during Hemin-Catalyzed Epoxidation of Terminal Alkenes, Journal of the American Chemical Society 117, 21-29.

53. Metcalfe, C. L., Ott, M., Patel, N., Singh, K., Mistry, S. C., Goff, H. M., and Raven, E. L. (2004) Autocatalytic Formation of Green Heme: Evidence for $\mathrm{H}_{2} \mathrm{O}_{2}$ Dependent Formation of a Covalent Methionine-Heme Linkage in Ascorbate Peroxidase, Journal of the American Chemical Society 126, 16242-16248.

54. Lee, H.-I., Dexter, A. F., Fann, Y.-C., Lakner, F. J., Hager, L. P., and Hoffman, B. M. (1997) Structure of the Modified Heme in Allylbenzene-Inactivated Chloroperoxidase Determined by Q-Band CW and Pulsed ENDOR, Journal of the American Chemical Society 119, 4059-4069.

55. Lowell P. Hager, D. R. M., Frederick S. Brown and Horst Eberwein (1966) Chloroperoxidase II. Utilization of Halogen Anions, Journal of Biological Chemistry 241, 1769-1777.

56. Morris, D. R., and Hager, L. P. (1966) Chloroperoxidase: I. Isolation and Properties of the Crystalline Glycoprotein Journal of Biological Chemistry 241, 1763-1768.

57. Wagner, G. C., Perez, M., Toscano, W. A., and Gunsalus, I. C. (1981) Apoprotein formation and heme reconstitution of cytochrome P-450cam, Journal of Biological Chemistry 256, 6262-6265. 
58. Wang, X., Tachikawa, H., Yi, X., Manoj, K. M., and Hager, L. P. (2003) Twodimensional NMR Study of the Heme Active Site Structure of Chloroperoxidase, Journal of Biological Chemistry 278, 7765-7774.

59. Griesinger, C., Otting, G., Wuethrich, K., and Ernst, R. R. (1988) Clean TOCSY for Proton Spin System Identification in Macromolecules, Journal of the American Chemical Society 110, 7870-7872.

60. Freeman, R., Pachler, K. G. R., and Mar, G. N. L. (1971) Quenching the Nuclear Overhauser Effect in NMR Spectra of Carbon-13, The Journal of Chemical Physics 55, 4586-4593.

61. Yu, C., Unger, S. W., and La Mar, G. N. (1986) NOE experiments for resonance and structure assignments of paramagnetic hemin derivatives, Journal of Magnetic Resonance 67, 346-350.

62. Bellingham, R. M., Gibbs, A. H., de Matteis, F., Lian, L. Y., and Roberts, G. C. (1995) Determination of the structure of an N-substituted protoporphyrin isolated from the livers of griseofulvin-fed mice, Biochem. J. 307, 505-512.

63. Lavallee, D. K., Kopelove, A. B., and Anderson, O. P. (1978) Structures and properties of N-methyltetraphenylporphyrin complexes. The crystal and molecular structure of chloro-N-methyl-.alpha.,.beta.,.gamma.,.delta.tetraphenylporphinatozinc(II) and the chelate effect of zinc(II), cobalt(II), and cadmium(II) N-methylporphyrin complexes, Journal of the American Chemical Society 100, 3025-3033.

64. McLaughlin, G. M. (1974) Crystal and molecular structure of a non-metallo, Nsubstituted porphyrin, 21-ethoxycarbonylmethyl-2,3,7,8,12,13,17,18octaethylporphyrin, Journal of the Chemical Society, Perkin Transactions 2, 136140 .

65. Kuhnel, K., Blankenfeldt, W., Terner, J., and Schlichting, I. (2006) Crystal Structures of Chloroperoxidase with Its Bound Substrates and Complexed with Formate, Acetate, and Nitrate, Journal of Biological Chemistry 281, 23990-23998.

66. Dexter, A. F., Lakner, F. J., Campbell, R. A., and Hager, L. P. (1995) Highly Enantioselective Epoxidation of 1,1-Disubstituted Alkenes Catalyzed by Chloroperoxidase, Journal of the American Chemical Society 117, 6412-6413. 


\section{CHAPTER III. THE ACTIVE SITE STRUCTURE OF SOYBEAN PEROXIDASE AS PROBED BY 1D AND 2D NUCLEAR OVERHAUSER ENHANCEMENT EFFECT}

\subsection{Introduction}

Plant peroxidases are a ubiquitous class of enzymes that catalyze the oxidation of a wide variety of organic and inorganic substrate molecules at the expense of hydrogen peroxide or other organic hydroperoxides. Plant peroxidases have found important and widespread medical and environmental applications, such as antigen conjugates for color visualization in immunoassays, catalysts in the treatment of various industrial wastes, and key component of biosensors in analytical diagnosis (1-3). The most widely studied plant peroxidase, horseradish peroxidase (HRP), has been historically used for such purposes because of its favorable stability and ease of isolation. However, the limited availability of the horseradish root extract combined with the shortage of alternative sources of the enzyme has made the use of HRP a relatively expensive practice (4-5). Therefore, an abundant and moderately inexpensive source of peroxidases for commercial utilization, especially for large scale environmental applications in the treatment of various industrial wastes to remove hazardous and/or toxic materials is highly desired.

Soybean peroxidase (SBP) is a very attractive candidate for such purposes as the enzyme shows a very similar reactivity profile with that of HRP (6). Meanwhile, SBP is readily obtained in reasonable yield from soybean seed hulls that are plentiful and inexpensive (7). Moreover, the conformational, thermal and kinetic stabilities, together with the catalytic efficiency of SBP are substantially higher than HRP (8-11). As a result, SBP has received an extensive amount of attention as a less expensive, more active, and more stable replacement of HRP for immunological, biocatalytical, biosensing 
applications during recent years (12-21).

Most of the previous investigations have been concerned with the physiological and biological functions of SBP. Less attention has been paid to the structural and chemical properties of the enzyme. The lack of structural information is a result of the difficulty encountered in obtaining suitable single crystals for X-ray diffraction, a common problem for most glycoprotein peroxidases (22). The presence of more than one closely-similar isozyme which leads to the microheterogeneity of the enzyme preparations is an additional problem for crystallization (23). Alternatively, the non-glycosylated recombinant SBP (rSBP) was expressed in Escherichia coli and its structure in the trisbound form was solved at a relative low resolution ( $2.8 \AA$, $88.2 \%$ overall completeness $)$ (6). Other structure studies of this enzyme include electronic absorption and resonance Raman (24), tryptophan fluorescence (25), Edman sequencing, and MALDI-TOF MS studies (26). Unfortunately, none of these studies were able to unequivocally reveal the structure of the active site cavity in native SBP. Despite this fact, structural information about many peroxidases from various sources has accumulated over the years through use of other indirect methods, such as computer modeling (27), sequence homology comparison to structurally characterized $\mathrm{C} c \mathrm{P}$ (28-32), and especially, NMR spectroscopy (33-34). The successful application of NMR spectroscopy to the elucidation of the heme active-site structural features of numerous peroxidases encouraged my interest in the project.

The primary objective of this study is to characterize the heme active-site structural properties of SBP through use of proton NMR spectroscopy, since information about the active-site structure of heme peroxidases is pivotal to the understanding of the structure- 
function relationship of this class of enzymes (35). The hyperfine-shifted signals of the heme protons were assigned unambiguously, and the NMR parameters and peak patterns of SBP are compared with those of HRP and CcP. The effects of the secondary metal ions, $\mathrm{Ca}^{2+}$, on the NMR features of SBP are discussed. Paramagnetically shifted signals are fully assigned through use of the 1D and 2D nuclear Overhauser enhancement (NOE) experiments and comparison with the spectral properties of $\mathrm{HRP}$ and $\mathrm{CcP}$.

\subsection{Materials and Methods}

Crude SBP was purchased from Bio-Research Products, Inc. (North Liberty, IA) and was further purified by passage through a DEAE Sephadex A-50 anion exchange column $(2.6 \times 20 \mathrm{~cm})$ followed by gel filtration (sephadex G-75, $2.6 \times 100 \mathrm{~cm}$ ). Proteins were eluted from the anion exchange column with a linear salt gradient consisting of 0 to $0.5 \mathrm{M}$ $\mathrm{NaCl}$ in $10 \mathrm{mM}$ phosphate buffer at $\mathrm{pH}$ 6.8. The procedure gives typical protein preparations with Rz values of 2.8 or even higher.

The concentration of the enzyme samples was determined spectrophotometrically using $\varepsilon_{403}=94.6 \mathrm{mM}^{-1} \mathrm{~cm}^{-1}$ for SBP (9). The molecular weight of SBP was determined to be $42 \mathrm{kD}$ through SDS electrophoresis. On the basis of the Soret band absorption, it was found that commercially purchased SBP preparations were about $60 \%$ pure by weight.

Enzymatic activity of SBP preparations (both native and $\mathrm{Ca}^{2+}$ depleted) was measured by both ABTS and guaiacol assays with each assay triplicated and the results averaged. ABTS is the registered trade name for 2,2'-azino-bis(3-ethylbenzthiazoline-6-sulfonic acid) diammonium salt, which has been suggested to be the most ideal substrate for peroxidase assays (36). Typically, the aqueous reaction mixture consisted of $1.5 \mathrm{mM}$ ABTS, $1.0 \mathrm{mM} \mathrm{H} \mathrm{H}_{2} \mathrm{O}_{2}$, and $0.1 \mu \mathrm{g} / \mathrm{ml} \mathrm{SBP}$. Hydrogen peroxide concentration was 
determined by its molar absorptivity of $39.4 \mathrm{M}^{-1} \mathrm{~cm}^{-1}$ at $240 \mathrm{~nm}$ (37). The reaction mixture was buffered at $\mathrm{pH} 6.0$ with $10 \mathrm{mM}$ phosphate and the reaction was started by the addition of $\mathrm{H}_{2} \mathrm{O}_{2}$. The progress of the reaction was monitored at $406 \mathrm{~nm}\left(\varepsilon_{406}=18.6\right.$ $\mathrm{mM}^{-1} \mathrm{~cm}^{-1}$ ) with the rate constant calculated from the first 15 seconds of the reaction. For the guaiacol assay, the reaction mixture consisted of $10 \mathrm{mM}$ sodium phosphate, $6.5 \mathrm{mM}$ guaiacol, $0.1 \mu \mathrm{g} \mathrm{SBP} / \mathrm{ml}$, and $0.06 \mathrm{mM} \mathrm{H}_{2} \mathrm{O}_{2}$. The reaction was carried out at $\mathrm{pH} 7.0$ and was monitored at $470 \mathrm{~nm}\left(\varepsilon=26.6 \mathrm{mM}^{-1} \mathrm{~cm}^{-1}\right)$ after addition of $\mathrm{H}_{2} \mathrm{O}_{2}$. The rate constant was calculated from the first 60 seconds of the reaction (38). All assays were carried out at room temperature.

The removal of $\mathrm{Ca}^{2+}$ from SBP was accomplished by the same procedure as described for HRP (39). Native SBP was incubated with $60 \mathrm{ml}$ of $6 \mathrm{M}$ guanidine hydrochloride containing $10 \mathrm{mM}$ EDTA at $\mathrm{pH} 7$ for 8 hours. The incubation mixture was then dialyzed three times against $4 \mathrm{~L}$ of $10 \mathrm{mM}$ phosphate buffer (pH 7) containing $10 \mathrm{mM}$ EDTA at 4 ${ }^{\circ} \mathrm{C}$ over a period of 24 hours, followed by dialysis at the same condition without EDTA. The reconstitution of $\mathrm{Ca}^{2+}$ was achieved by addition of a 100 -fold excess of $\mathrm{CaCl}_{2}$ to the calcium-depleted SBP $(0.7 \mathrm{mM})$ in $10 \mathrm{mM}$ Tris buffer at $\mathrm{pH}$ 6.8. Incubation of the native SBP with excess $\mathrm{Ca}^{2+}$ showed no uptake of extra $\mathrm{Ca}^{2+}$ into the protein as determined by atomic absorption analysis, indicating that all calcium-binding sites were occupied. Tris buffers were used in calcium reconstitution experiments in order to minimize interference from formation of insoluble or refractory calcium phosphate which might otherwise be experienced with phosphate buffers.

The heme group of SBP was easily extracted from the native enzyme by the reported 2-butanone procedure (40). It was found that the prosthetic group of SBP is indeed an 
iron protoporphyrin IX (Figure 1.1B) as was earlier suggested, since the NMR spectrum of the extracted prosthetic group is identical to that of the standard protoheme. Therefore, no efforts were made to reconstitute the standard iron protoporphyrin IX into the apoprotein of SBP.

Proton NMR spectra of both native and calcium-depleted SBP samples were recorded at $20,25,30,35,40{ }^{\circ} \mathrm{C}$ on a Bruker Avance 600 (Ultrashield) NMR spectrometer operation at a proton frequency of 599.93 MHz. Protein concentrations in NMR samples varied from 2.5 to $2.8 \mathrm{mM}$ in either $\mathrm{H}_{2} \mathrm{O}$ or $\mathrm{D}_{2} \mathrm{O}$ buffers ( $10 \mathrm{mM}$ phosphate, $\mathrm{pH}$ 6.8). The NMR samples in $\mathrm{D}_{2} \mathrm{O}$ were prepared by at least five times of isotope exchange of the protein solution with $\mathrm{D}_{2} \mathrm{O}$ buffer at $\mathrm{pH} 6.8$ (uncorrected for any isotope effect). The isotope exchanges were carried out in a $2.0 \mathrm{ml}$ Millipore Centricon YM-30 centrifugal filter unit at $4{ }^{\circ} \mathrm{C}$. The ${ }^{1} \mathrm{H}$ NMR spectra of the high-spin ferric native, calcium-depleted, and calcium-reconstituted SBP were collected with a $125 \mathrm{kHz}$ spectral width, 16384 data points, and a repetition rate of approximately 15 per second. Generally, 20,000 to 60,000 scans were needed to obtain a decent spectrum due to the high spin nature of the protein. Free induction decays were exponentially multiplied by a line broadening factor of 100 $\mathrm{Hz}$ before Fourier transformation to improve the signal-to-noise ratio of the spectra.

The low-spin cyanide-adducts of the protein were prepared by adding a 20 fold excess of freshly prepared $\mathrm{KCN}$ to the high-spin enzyme samples. The NMR spectra of the ferric low-spin forms were obtained by collecting 1000-2000 scans with $4 \mathrm{~K}$ data points over a $62.5 \mathrm{kHz}$ bandwidth, and a repetition time of 0.2 second with solvent presaturation during relaxation delay. The spectra were obtained by multiplication of the free induction decays with a 20 to $40 \mathrm{~Hz}$ apodization. Fast repetition spectra of the low- 
spin protein-cyanide complexes were also recorded with a $125 \mathrm{kHz}$ sweep width, 2048 data points, and a repetition rate of $10 \mathrm{~ms}$ to detect the fast relaxing proton signals which are intrinsically broad. Chemical shift values at different temperatures for both the ferric high- and low-spin forms of SBP are referenced to the residual HDO signal according to equation $11(41)$ :

$$
\delta_{(\mathrm{HDO})}=7.835-T(\mathrm{~K}) / 96.9
$$

The spin-lattice relaxation times $\left(T_{1}\right)$ of the hyperfine-shifted protons for the cyanide complex of SBP were measure by the standard inversion-recovery pulse sequence. A repetition rate of 3.3 per second and a $90^{\circ}$ transmitter pulse of $7.6 \mu$ s was used. The $T_{1}$ values were estimated from the null point with the relationship of $T_{1}=\tau / \ln 2$.

The NOE experiments on the cyanide complex of the protein were performed by irradiation of the resonance of interest prior to the $90^{\circ}$ pulse. Typically, the peak of interest is irradiated for $100 \mathrm{~ms}$ with a weak decoupler power of $65 \mathrm{~dB}$ and a repetition time of 0.2 second to obtain steady state NOEs. The buildup of NOE is time dependent and is expressed by equation 12 for an isolated two-spin system (42):

$$
\eta_{i}(t)=\frac{\sigma_{i j}}{\rho_{i}}\left(1-\mathrm{e}^{-\rho_{i} t}\right)
$$

where, $\rho_{i}^{-1}=\mathrm{T}_{1 i}$, the selective spin-lattice relaxation time of $\mathrm{H}_{i}, t$ is the duration of the saturating pulse on $\mathrm{H}_{j}$, and $\sigma_{i j}$ is the cross-relaxation between $\mathrm{H}_{i}$ and $\mathrm{H}_{j}$. In the high-field case $\left(\omega^{2} \tau_{\mathrm{c}}^{2} \gg 1\right)$, the cross-relaxation is given by equation $13(42)$ :

$$
\sigma_{i j}=\frac{-\hbar^{2} \gamma^{4}}{10 r_{i j}^{6}} \tau_{\mathrm{c}}
$$

where $\tau_{\mathrm{c}}$ is the reorientation time of the vector defined by $\mathrm{H}_{i}$ and $\mathrm{H}_{j}$, and $r_{i j}$ is the length of this vector. For short irradiation times $(<30 \mathrm{~ms}) \eta_{i}$ takes the form as in equation 14: 


$$
\eta_{i}(t)=\sigma_{i j} t
$$

and is independent of the spin-lattice relaxation time of the nucleus.

2D phase-sensitive NOESY and magnitude COSY spectra for the cyanide-bound derivative of SBP were recorded at $600 \mathrm{MHz}$ by using presaturation to eliminate the residual water signal. All pulse sequences utilized here employed single $90^{\circ}$ hard pulses to maximize excitation bandwidth. NOESY spectra with a spectral width of about $60 \mathrm{ppm}$ were recorded at 35 and $40{ }^{\circ} \mathrm{C}$ with a repetition rate of $5 \mathrm{~s}^{-1}$ and a mixing time of $20 \mathrm{~ms}$ to avoid spin diffusion. A total of 2000 experiments were collected with 1024 data points in the F2 direction. The COSY spectra were collected at $25^{\circ} \mathrm{C}$ with other parameters similar to NOESY. All 2D spectra data consist of 4096 data points in the acquisition dimension and of 1024 in the indirect dimension. The data were multiplied in both dimensions and were zero-filled and Fourier-transformed to obtain $1024 \times 1024$ real data points.

\subsection{Results}

\subsubsection{Proton NMR of the Native Ferric High-Spin SBP}

The proton NMR spectra of the native ferric high-spin SBP in $\mathrm{D}_{2} \mathrm{O}$ solution are shown in Figure 3.1. No additional hyperfine shifted signals were detected when the experiments were carried out in $\mathrm{H}_{2} \mathrm{O}$ solution. A very broad signal " $\mathrm{A}$ " at $98 \mathrm{ppm}$, which was observed for other hemoproteins $(34,43-45)$, was also detected for SBP. Because this broad, far downfield shifted signal has been assigned to the $\mathrm{N}-\delta_{1}-\mathrm{H}$ of the proximal histidine (Figure 3.2) in several hemoproteins, the presence of this peak is a strong indication that the proximal ligand in SBP is the same as that in most traditional hemoproteins, which is consistent with the crystal structure of rSBP. Signal "A" is

exchangeable and its exchange behavior varies with different proteins. For manganese 
and lignin peroxidase this signal disappear soon after the sample is placed in $\mathrm{D}_{2} \mathrm{O}(34$, 45), while for HRP the signal maintains its intensity even after the protein is dissolved in $\mathrm{D}_{2} \mathrm{O}$ for several weeks (43). The exchange rate of this signal in SBP is very similar to that of the corresponding signal in HRP, as the signal remains detectable even after 3 weeks in $\mathrm{D}_{2} \mathrm{O}$ buffer.

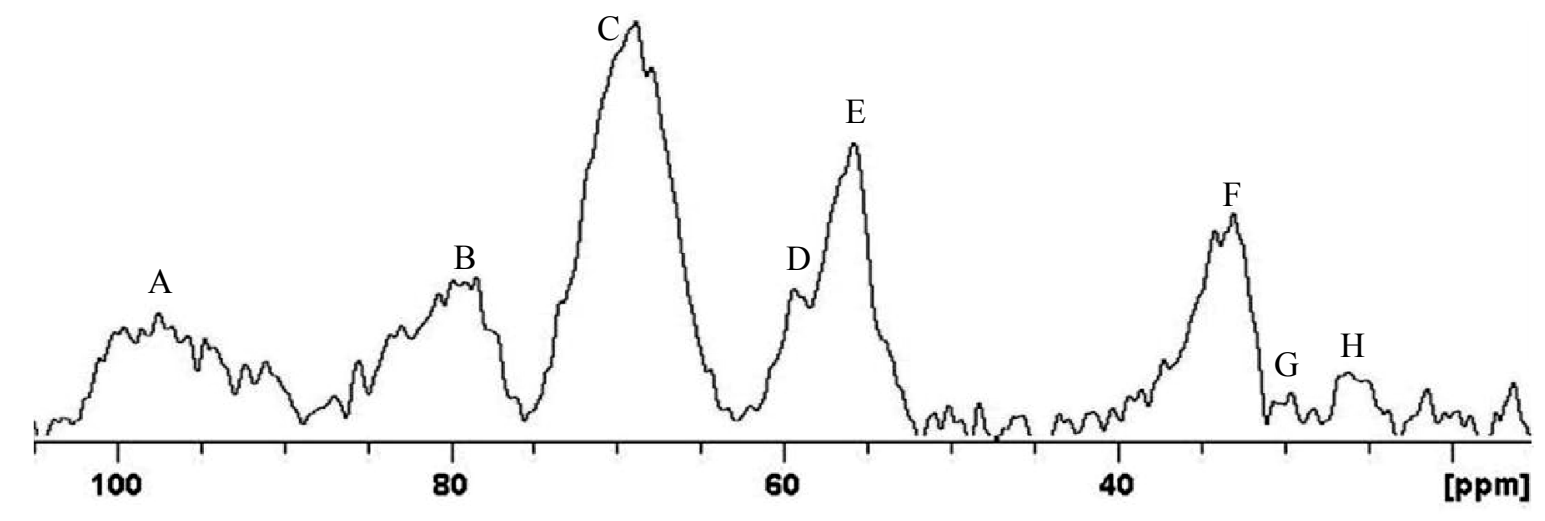

Figure 3.1 The $600 \mathrm{MHz}$ proton NMR spectra of $2.5 \mathrm{mM}$ native high-spin SBP in $10 \mathrm{mM}$ phosphate buffer at $\mathrm{pH} 6.8$ and $298 \mathrm{~K}$.

\subsubsection{Proton NMR of the Cyanide Adduct of Native SBP}

The proton NMR spectrum of the cyanide-ligated SBP complex in $\mathrm{D}_{2} \mathrm{O}$ solution is shown in Figure 3.3A. Sharp lines for the paramagnetically shifted signals were observed since the $\mathrm{CN}^{-}$adducts of all heme peroxidases contain low-spin ferric iron $(\mathrm{S}=1 / 2)$. Additional peaks were detected when the spectrum was recorded in $\mathrm{H}_{2} \mathrm{O}$ solution (Figure 3.3B). The spectral features of SBP- $\mathrm{CN}^{-}$display a high degree of similarity to those of HRP-CN ${ }^{-}$and $\mathrm{CcP}-\mathrm{CN}^{-}$shown in Figures $3.3 \mathrm{C}$ and 3.3D. The close similarity of the proton NMR spectra of these peroxidases is an indication that they share the same protoheme IX prosthetic group. 


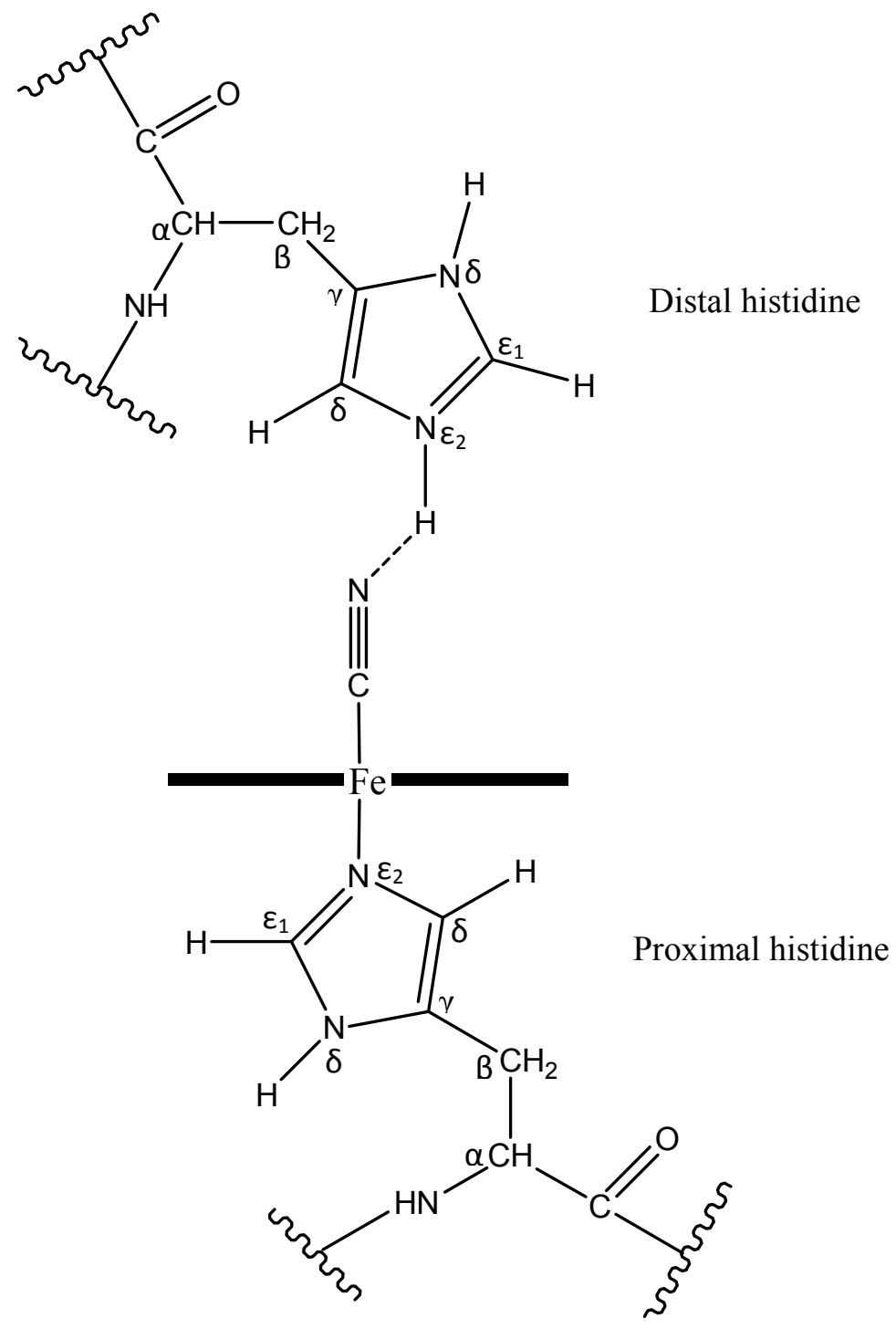

Figure 3.2 The schematic representation of the active site structure of the cyanide-ligated form of most heme peroxidases showing the presence of both the proximal and distal histidine. The protoporphyrin plane is represented by the two bold horizontal bars.

Assignment of the hyperfine shifted signals for the SBP-CN${ }^{-}$complex was achieved through comparison with the assignments made for $\mathrm{HRP}-\mathrm{CN}^{-}$and $\mathrm{C} c \mathrm{P}-\mathrm{CN}^{-}$(Table 3.1) (34, 46-52) and examination of the active site structure revealed by the rSBP crystal structure (6) with confirmation through one- and two-dimensional NOE measurements as well as through bond connectivities (COSY). The 1D NOE experiments were carried out 
by irradiation of the hyperfine shifted signals with a weak decoupler power for a short period of time, typically $100 \mathrm{~ms}$. Some NOE difference spectra are shown in Figures 3.4(B to E), and Figures 3.5(B to F).

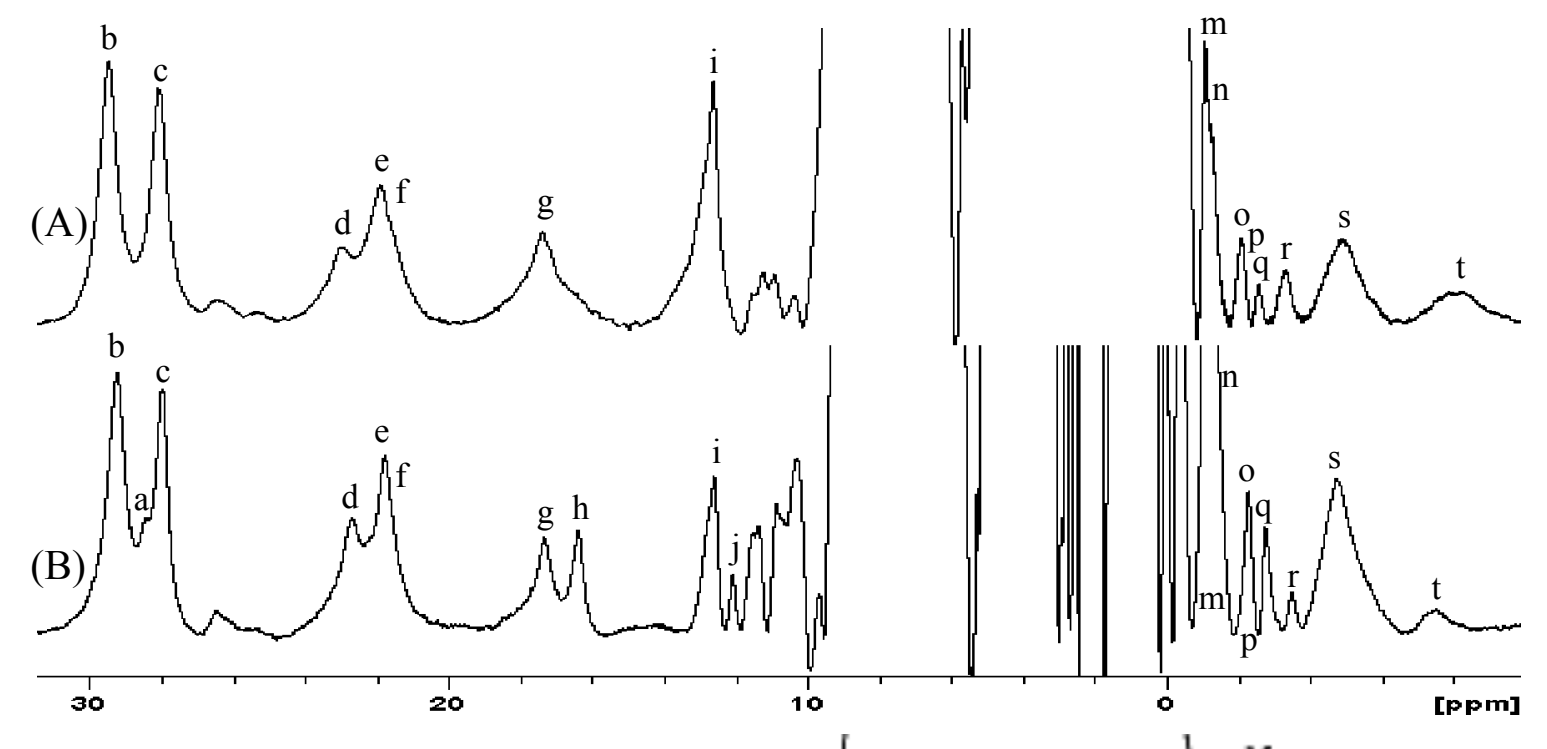

(C)
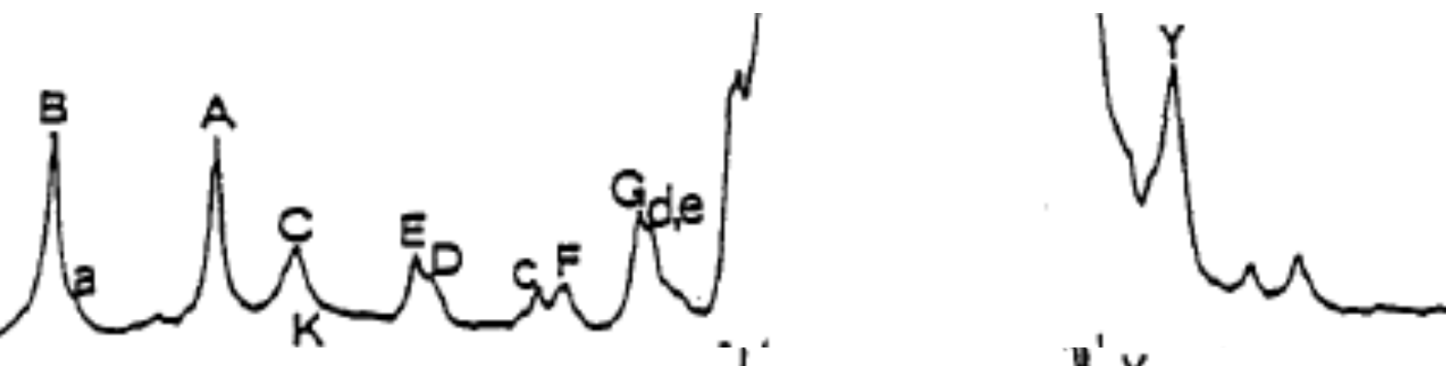

(D)
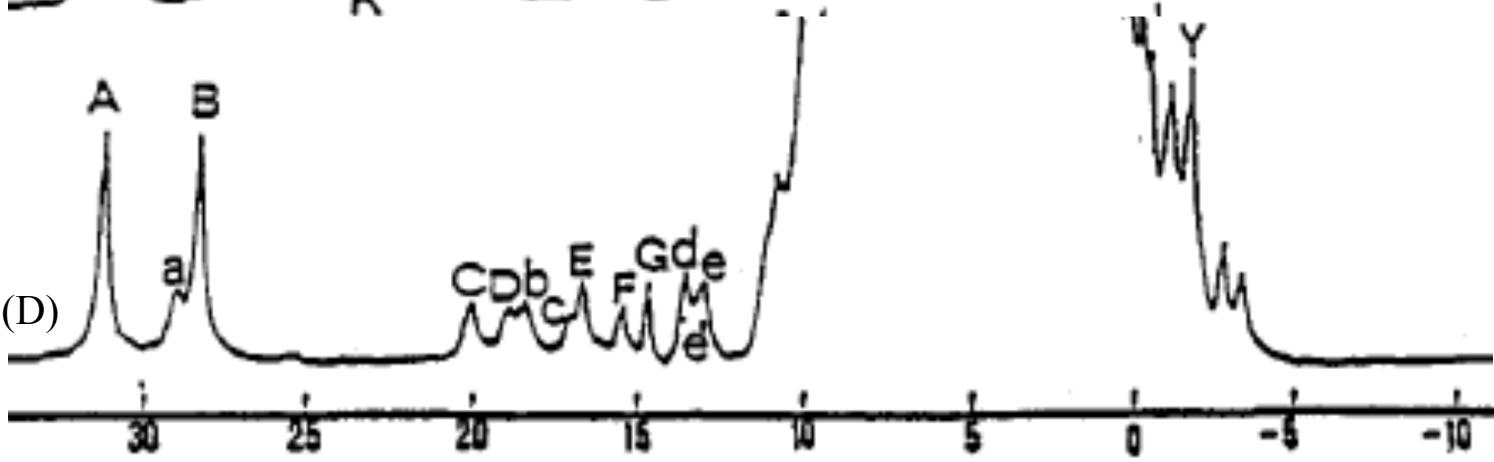

Figure 3.3 The proton NMR spectra of the low-spin protein-cyanide complexes. (A) SBP in $10 \mathrm{mM}$ phosphate $\mathrm{D}_{2} \mathrm{O}$ buffer at $\mathrm{pH} 6.8,298 \mathrm{~K}$.; (B) SBP in the same buffer with $90 \% \mathrm{H}_{2} \mathrm{O}$ and $10 \% \mathrm{D}_{2} \mathrm{O}$ at $298 \mathrm{~K}$; (C) HRP in the same buffer with $90 \% \mathrm{H}_{2} \mathrm{O}$ and $10 \% \mathrm{D}_{2} \mathrm{O}$ at $\mathrm{pH} 6.3,301 \mathrm{~K}$; and (D) $\mathrm{CcP}$ in the same buffer with $90 \% \mathrm{H}_{2} \mathrm{O}$ and $10 \% \mathrm{D}_{2} \mathrm{O}$ at $\mathrm{pH} 6.3,301 \mathrm{~K}$. Spectra (C) and (D) are from ref (34). 
The spectrum of the low-spin SBP cyanide complex collected with a rapid repetition rate at $298 \mathrm{~K}$ is shown in Figure 3.6. Two broad resonances (peaks "z" and " $y$ " each of single proton intensity) were observed in the downfield and upfield region, respectively. Peak " $y$ " is poorly resolved from the methyl peak "b" and shows only as a shoulder of peak "b" at $298 \mathrm{~K}$. These two fast relaxing signals were also detected earlier for several other low-spin heme peroxidases, such as $\mathrm{CMP}_{-} \mathrm{CN}^{-}(43,52)$.

Shown in Figure 3.7 and 3.8 are the NOESY spectra of SBP-CN $-\mathrm{CN}^{-} \mathrm{D}_{2} \mathrm{O}$ buffer with a mixing time of $20 \mathrm{~ms}$ collected at $308 \mathrm{~K}$ and $313 \mathrm{~K}$, respectively. The NOESY spectra of SBP-CN ${ }^{-}$in $90 \% \mathrm{H}_{2} \mathrm{O}$ and $10 \% \mathrm{D}_{2} \mathrm{O}$ buffer with a mixing time of $20 \mathrm{~ms}$ collected at 323 $\mathrm{K}$ is shown in Figure 3.9. The NOESY spectra provide a greatly improved resolution, especially for overlapped peaks which are hard to differentiate from 1D NOE results. The clear NOESY connectivities and the results from scalar (COSY; Figure 3.10) connectivities prove the validity of the NOE connectivities observed in 1D NOE experiments and lead to the proposed assignments for all of the hyperfine shifted protons from the heme active site in SBP-CN. Furthermore, resonances from protons that are near to the heme active center but are buried in the diamagnetic envelope were also revealed from NOESY spectra as shown in Table 3.2. The suggested assignments for hyperfine shifts are further verified by the Curie intercepts predicted from the temperature dependence of the resonances (Figure 3.11).

\subsubsection{Role of Endogenous Calcium}

Atomic absorption spectroscopic analysis showed that native SBP contains an

average of $2.9 \mathrm{Ca}^{2+}$ ions per molecule of SBP based on the Soret absorption of the protein (Wang, unpublished results). This result is in complete agreement with the results 
Table 3.1 ${ }^{\mathrm{a}}$ Proton NMR chemical shift (ppm), $\mathrm{T}_{1}(\mathrm{~ms})$, and Curie plots intercept (ppm) values of the isotropically shifted resonances for the low-spin cyanide derivatives of SBP, $\mathrm{HRP}$ and $\mathrm{C} c \mathrm{P}$.

\begin{tabular}{|c|c|c|c|c|c|c|c|c|c|}
\hline \multirow[b]{2}{*}{ Peak } & \multicolumn{3}{|c|}{$\mathrm{SBP}_{-\mathrm{CN}^{-}}$} & \multicolumn{3}{|c|}{ HRP-CN ${ }^{-}$} & \multicolumn{2}{|c|}{$\mathrm{C} c \mathrm{P}-\mathrm{CN}^{-}$} & \multirow{2}{*}{ Assignment } \\
\hline & Shift & $\mathrm{T}_{1}$ & Int & Shift & $\mathrm{T}_{1}$ & $\operatorname{Int}^{\mathrm{c}}$ & Shift & $\mathrm{T}_{1}$ & \\
\hline & ppm & $m s$ & ppm & ppm & $m s$ & ppm & ppm & $m s$ & \\
\hline$a^{b}$ & 26.7 & --- & --- & 31.0 & --- & --- & 28.4 & 7 & His $42 \mathrm{~N}-\varepsilon_{2}-\mathrm{H}$ \\
\hline $\mathrm{b}$ & 28.0 & 108 & -3.9 & 31.0 & 57 & -0.8 & 27.6 & 40 & $8-\mathrm{CH}_{3}$ \\
\hline $\mathrm{c}$ & 26.6 & 101 & -4.8 & 26.0 & 44 & -1.0 & 30.6 & 39 & $3-\mathrm{CH}_{3}$ \\
\hline d & 21.4 & 58 & -7.8 & 23.7 & 21 & -9.8 & 19.4 & 34 & His $169 \mathrm{C}-\beta-\mathrm{H}$ \\
\hline $\mathrm{d}^{\prime}$ & 21.4 & --- & --- & $20.8^{\mathrm{c}}$ & --- & --- & 15.8 & --- & His $169 \mathrm{C}-\delta_{2}-\mathrm{H}$ \\
\hline $\mathrm{e}$ & 21.4 & 94 & 7.0 & 19.6 & 83 & 7.6 & 18.3 & 45 & 4-vinyl Ha \\
\hline $\mathrm{f}$ & 20.7 & 129 & 1.9 & 20.1 & 96 & 5.7 & 16 & 60 & 7-propionate $\mathrm{H} \alpha$ \\
\hline $\mathrm{g}$ & 16.3 & 94 & -5.4 & 15.6 & 41 & -4.7 & 14.8 & 34 & His $169 C-\beta^{\prime}-H$ \\
\hline$h^{b}$ & 16.2 & --- & 11.3 & 16.3 & 260 & --- & 16.5 & --- & His $42 \mathrm{~N}-\delta_{1}-\mathrm{H}$ \\
\hline $\mathrm{i}$ & 12.2 & 87 & 3.8 & 13.3 & 42 & 3.6 & 14 & 67 & His $42 \mathrm{C}-\varepsilon_{1}-\mathrm{H}$ \\
\hline$i^{\prime}$ & 12.2 & --- & --- & 12.9 & 238 & 1.0 & 12.9 & 110 & His $169 \mathrm{~N}_{\mathrm{pep}} \mathrm{H}$ \\
\hline$j^{b}$ & 12.1 & --- & 10.7 & $9.9^{c}$ & --- & 9.9 & 10.2 & --- & His $169 \mathrm{~N}-\delta_{1}-\mathrm{H}$ \\
\hline $\mathrm{m}$ & -0.9 & 302 & 1.8 & $-1.1^{c}$ & --- & 5.8 & --- & --- & 2-vinyl $\mathrm{H}_{\beta}$-cis \\
\hline $\mathrm{n}$ & -1.2 & 302 & 0.1 & $-2.9^{c}$ & --- & 1.3 & 85 & --- & Leu $243 \delta_{2}-\mathrm{CH}_{3}$ \\
\hline o & -1.7 & 216 & 6.3 & -1.8 & --- & 4.4 & --- & --- & 4-vinyl $\mathrm{H}_{\beta}$-trans \\
\hline $\mathrm{p}$ & -2.1 & 216 & 7.0 & $-2.8^{\mathrm{c}}$ & --- & 5.5 & --- & --- & 4-vinyl $\mathrm{H}_{\beta}$-cis \\
\hline $\mathrm{q}$ & -2.5 & 115 & 5.7 & $-2.3^{c}$ & --- & 5.4 & --- & --- & 6-propionate $\mathrm{H} \beta$ \\
\hline $\mathrm{r}$ & -2.8 & 115 & 8.2 & $-2.2^{\mathrm{c}}$ & --- & 6.6 & --- & --- & 2-vinyl $\mathrm{H}_{\beta}$-trans \\
\hline $\mathrm{s}$ & -4.6 & 80 & 3.5 & $-4.8^{c}$ & --- & 4.2 & 90 & --- & $\operatorname{Arg} 38 \mathrm{H} \beta$ \\
\hline $\mathrm{t}$ & -7.7 & 50 & 2.4 & $-6.5^{c}$ & --- & 0.5 & 45 & --- & $\operatorname{Arg} 38 \mathrm{H} \delta$ \\
\hline $\mathrm{y}$ & 30.1 & --- & --- & --- & --- & --- & --- & --- & \\
\hline $\mathrm{z}$ & -28.3 & --- & --- & -29.9 & 2.7 & --- & -20.6 & 2.5 & His $169 \mathrm{C}-\varepsilon_{1}-\mathrm{H}$ \\
\hline
\end{tabular}

\footnotetext{
${ }^{a}$ Results for HRP-CN- and $\mathrm{CcP}-\mathrm{CN}$ - are from ref (34) at 301K. Results of SBP are from NMR spectra collected at $313 \mathrm{~K}$.

${ }^{\mathrm{b}}$ Indicates exchangeable protons.

${ }^{\mathrm{c}}$ From ref (47-48) measured at $323 \mathrm{~K}$.
} 


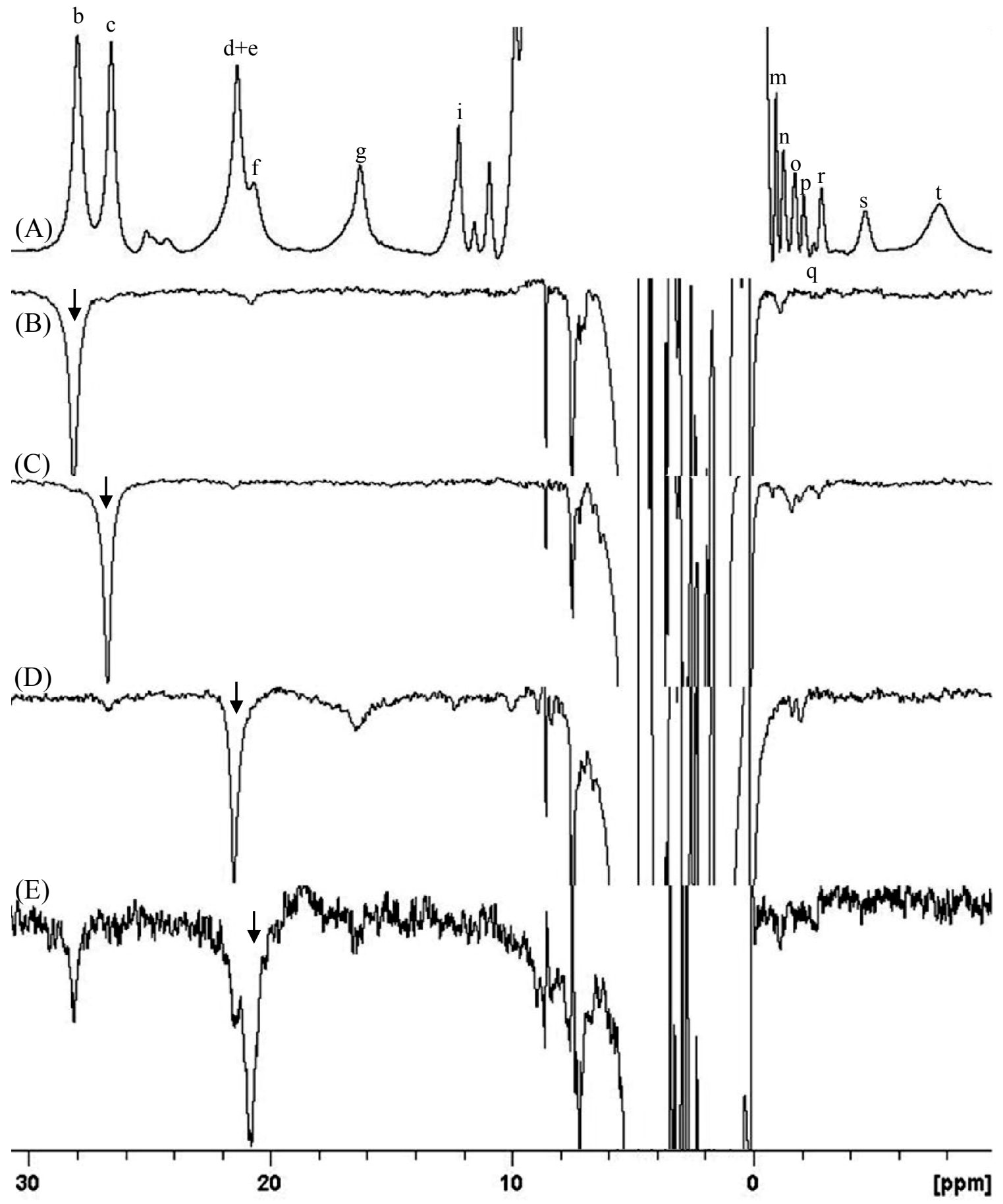

Figure 3.4 The 600-MHz proton NMR spectrum of (A) $2.8 \mathrm{mM}$ low-spin cyanide-ligated form of SBP in $10 \mathrm{mM}$ phosphate $\mathrm{D}_{2} \mathrm{O}$ solution at $313 \mathrm{~K}, \mathrm{pH} 6.8$; note peak "d" and "e" overlap at $21.4 \mathrm{ppm}$. (B) trough (E) are the NOE difference spectra obtained upon irradiation of the downfield shifted signals "b", "c", "d+e", and "f", respectively. The irradiated peaks are indicated by a downward arrow in each difference spectrum. 


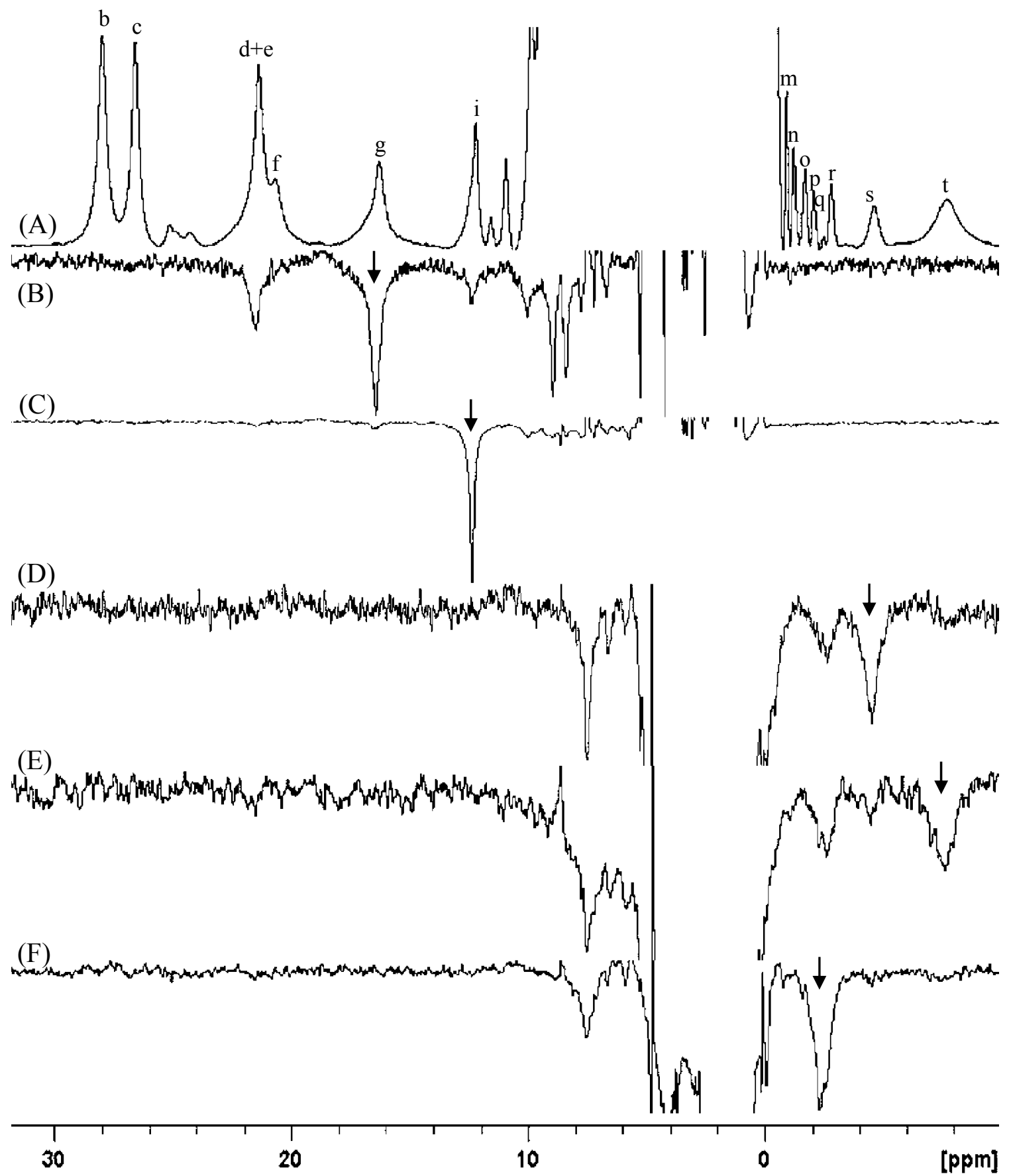

Figure 3.5 The 600-MHz proton NMR spectrum of (A) $2.8 \mathrm{mM}$ low-spin cyanide-ligated form of SBP in $10 \mathrm{mM}$ phosphate $\mathrm{D}_{2} \mathrm{O}$ solution at $313 \mathrm{~K}, \mathrm{pH} 6.8$; (B) trough (F) are the NOE difference spectra obtained upon irradiation of the downfield shifted signals "g", "i", "s", "t" and "q", respectively. The irradiated peaks are indicated by a downward arrow in each difference spectrum. 


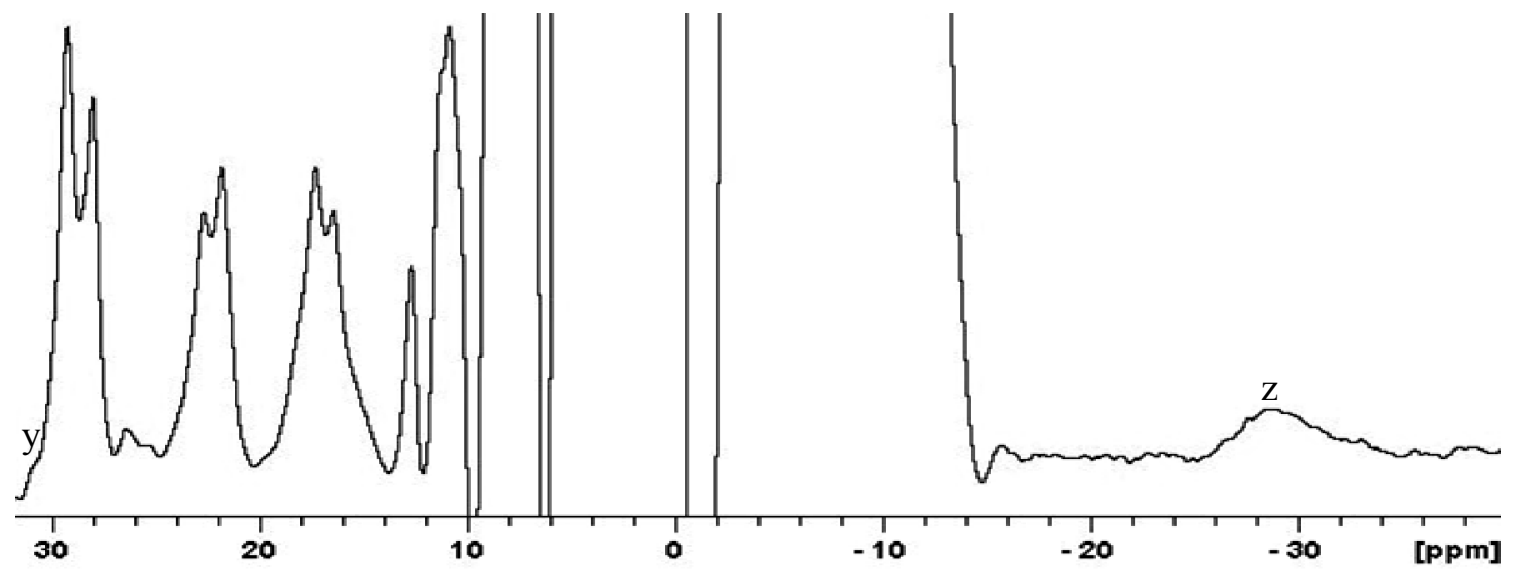

Figure 3.6 The $600-\mathrm{MHz}$ proton NMR spectrum of the ferric low-spin $\mathrm{SBP}^{-\mathrm{CN}^{-}}$complex in $10 \mathrm{mM}$ phosphate $\mathrm{D}_{2} \mathrm{O}$ buffer collected with a rapid repetition rate at $298 \mathrm{~K}, \mathrm{pH} 6.8$.

obtained for Coprinus cinereus peroxidase (53) and Coprinus macrorhizus peroxidase. However, different results have been reported for an average of $2 \mathrm{Ca}^{2+}$ per molecule content of $\operatorname{rSBP}(6)$ and $\operatorname{HRP}(39,54)$. Nevertheless, results of as high as $4 \mathrm{Ca}^{2+}$ per molecule of HRP have also been reported (55). Despite the controversial results reported for the calcium content in HRP, all the above mentioned references have presented the complete removal of the calcium ion from the protein by incubating the enzyme with guanidine hydrochloride and EDTA.

Enzymatic activity measurements indicated that elimination of the guanidine-EDTA removable calcium ion from SBP decreased the initial protein activity by $20 \%$ for the ABTS assay (from 338 to $272 \mu$ mole/min'mg SBP) and $31 \%$ for the guaiacol assay (from 565 to $390 \mu \mathrm{mole} / \mathrm{min} \cdot \mathrm{mg} \mathrm{SBP})$. Incubation of the calcium "depleted" SBP with excess of $\mathrm{CaCl}_{2}$ regained the full activity of the enzyme. However, in the case of HRP-C, removal of the bound calcium resulted in approximately $60 \%$ drop of the initial activity of the protein (39). 


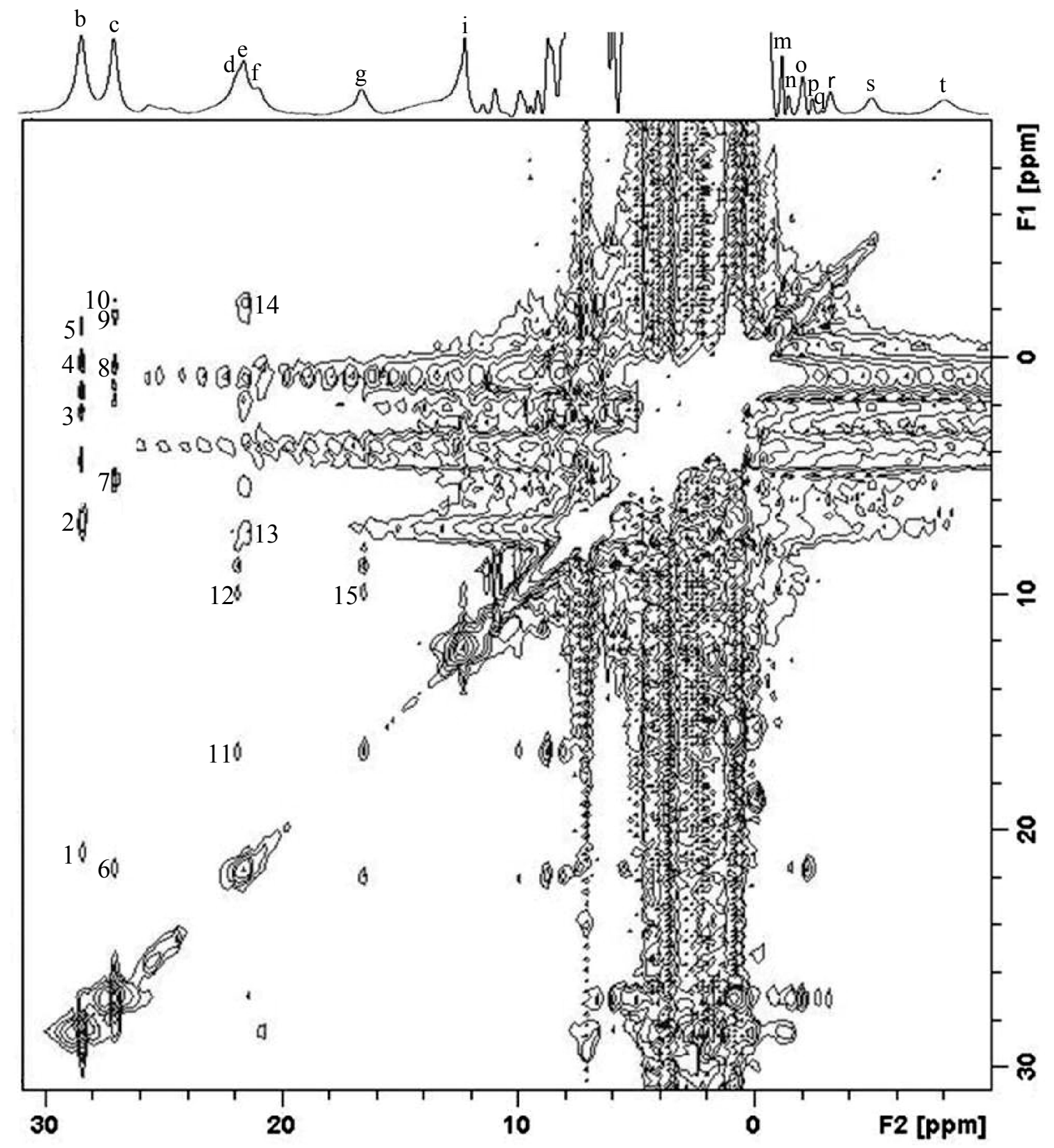

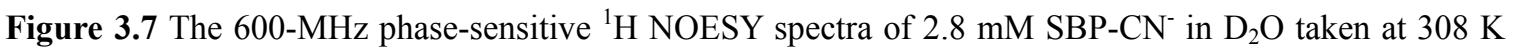
with a mixing time of $20 \mathrm{~ms}$. Cross-peak assignments are as follows: $1,8-\mathrm{CH}_{3}: 7-\mathrm{H} \alpha ; 2,8-\mathrm{CH}_{3}: \mathrm{H}-\delta_{\text {meso }} ; 3$, 8- $\mathrm{CH}_{3}: 7-\mathrm{H} \beta ; 4,8-\mathrm{CH}_{3}: 7-\mathrm{H} \beta \prime ; 5,8-\mathrm{CH}_{3}: \delta_{2}-\mathrm{CH}_{3}$ Leu243 (4.13 $\AA$ ); 6, 3- $\mathrm{CH}_{3}: 4-\mathrm{H} \alpha ; 7,3-\mathrm{CH}_{3}:$ ring-H Phe41 ( 4.5 $\AA$ ); 8, 3- $\mathrm{CH}_{3}: \mathrm{H}-\alpha_{\text {meso }} ; 9,3-\mathrm{CH}_{3}: 4-\mathrm{H} \beta_{\text {trans }} ; 10,3-\mathrm{CH}_{3}: 4-\mathrm{H} \beta_{c i s} ; 11, \mathrm{H} \beta$ His 169:H $\beta$ ' His 169; $12, \mathrm{H} \beta$ His169:H $\alpha$ His 169; 13, 4-H $\alpha: \mathrm{H}-\beta_{\text {meso }} ; 14,4-\mathrm{H} \alpha: 4-\mathrm{H} \beta_{\text {trans }} ; 15, \mathrm{H} \beta$ ' His169:H $\alpha$ His169; The numbers in parenthesis are the distances between protons that give rise to the cross-peak measured in rSBP crystal structure. 


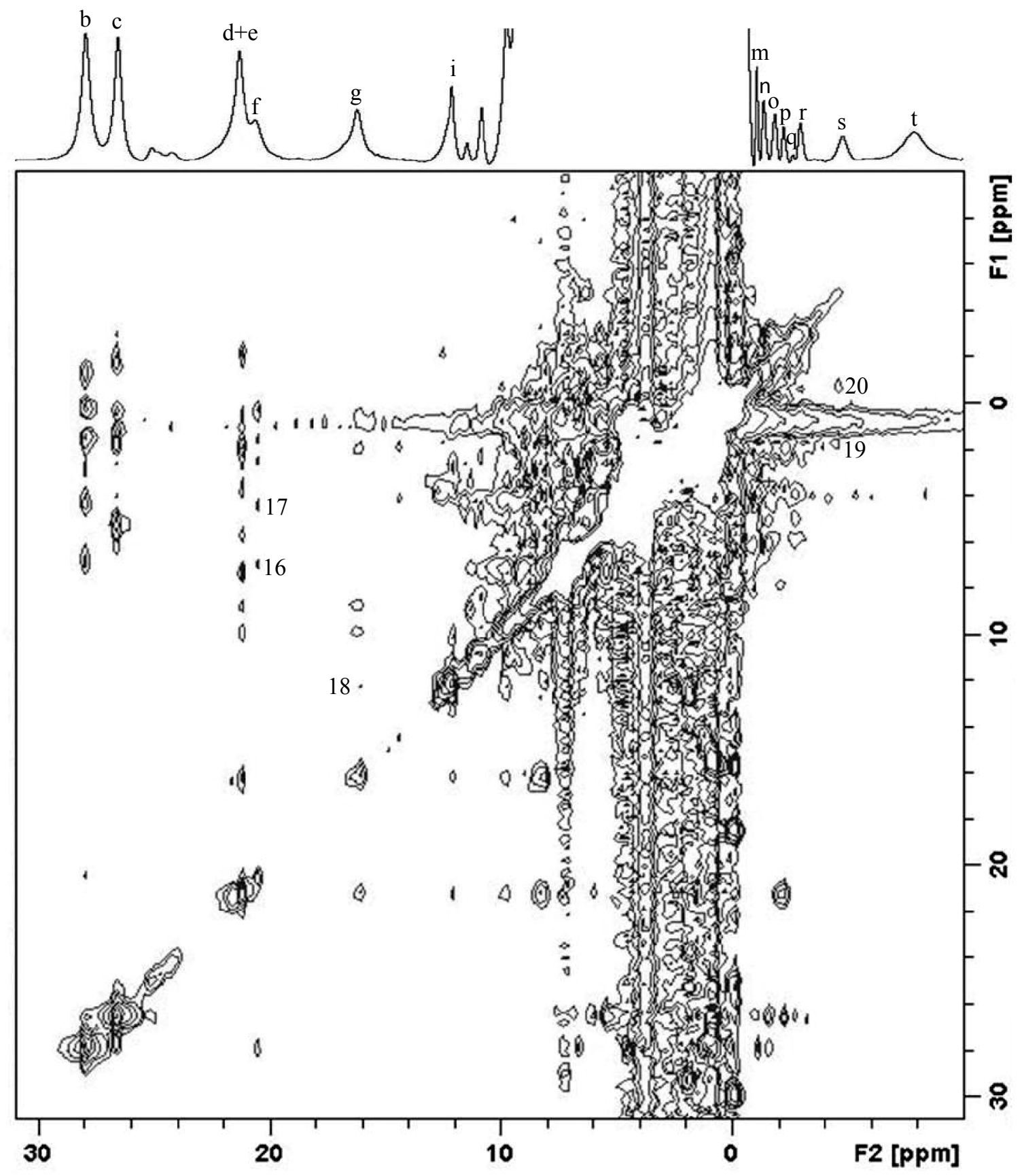

Figure 3.8 The $600-\mathrm{MHz}$ phase-sensitive ${ }^{1} \mathrm{H}$ NOESY spectra of $2.8 \mathrm{mM} \mathrm{SBP-CN}^{-}$in $\mathrm{D}_{2} \mathrm{O}$ taken at $313 \mathrm{~K}$ with a mixing time of 20 ms. Cross-peak assignments in addition to those of Figure 3.7 are as follows (numbering continuously from Figure 3.7): 16, 7-H $\alpha: 7-\mathrm{H} \alpha ; 17,7-\mathrm{H} \alpha: \mathrm{H}-\gamma_{\text {meso }} ; 18, \mathrm{H} \beta$ ' His 169: $\mathrm{N}_{\mathrm{PEP}} \mathrm{H}$ His 169; $19, \mathrm{H} \beta \operatorname{Arg} 38: \mathrm{H} \delta{ }^{\prime} \operatorname{Arg} 38 ; 20, \mathrm{H} \beta \operatorname{Arg} 38: \mathrm{H} \beta$ ' Arg38. 


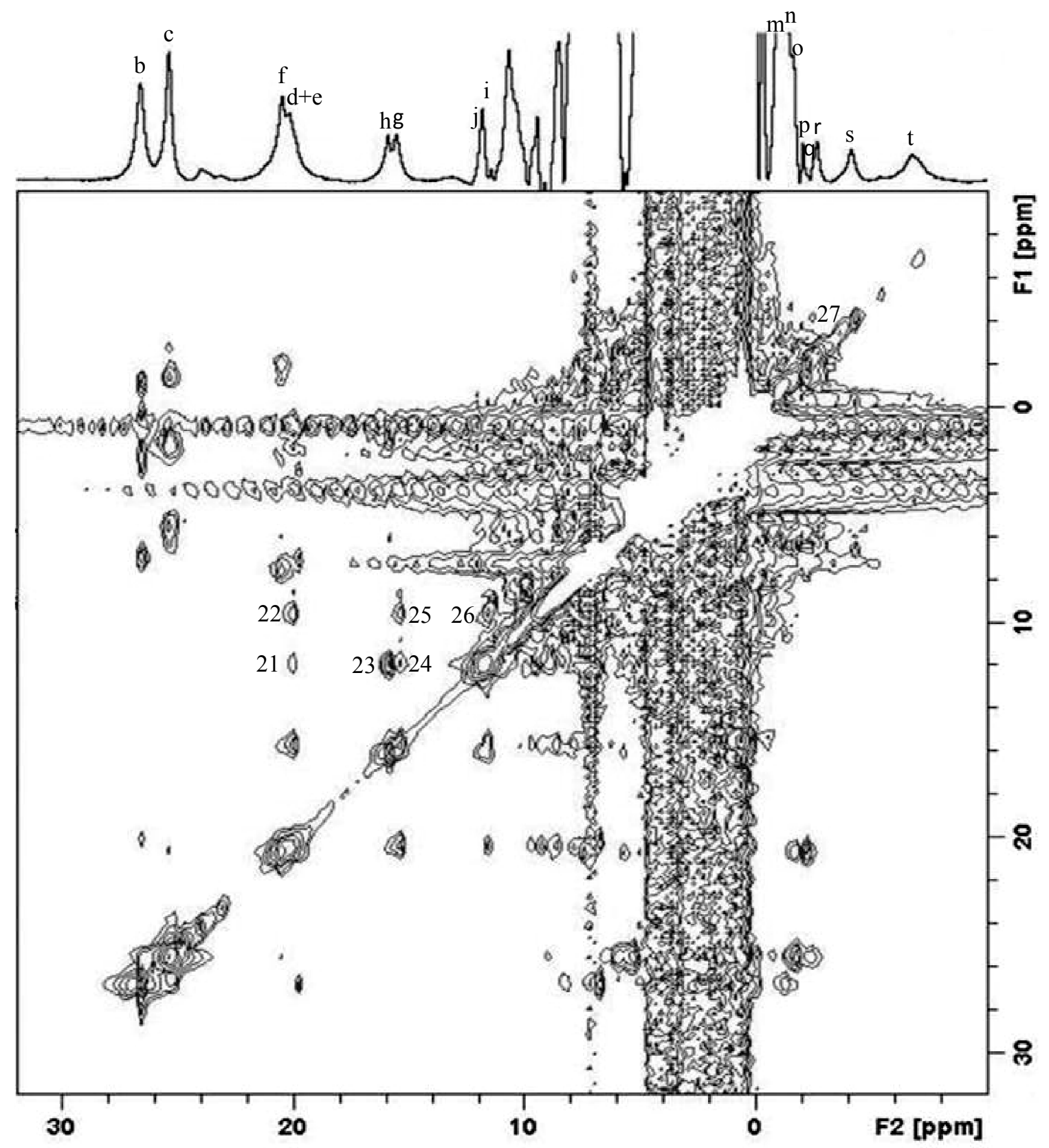

Figure 3.9 The 600-MHz phase-sensitive ${ }^{1} \mathrm{H}$ NOESY spectra of $2.5 \mathrm{mM} \mathrm{SBP}^{-\mathrm{CN}}{ }^{-}$in $90 \% \mathrm{H}_{2} \mathrm{O}$ and $10 \%$ $\mathrm{D}_{2} \mathrm{O}$ taken at $323 \mathrm{~K}$ with a mixing time of $20 \mathrm{~ms}$. Cross-peak assignments in complete agreement with 1D NOE assignments for distal and proximal histidine protons are as follows (numbering continuously from

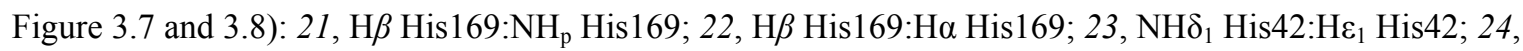
$\mathrm{H} \beta$ ' His169: $\mathrm{NH}_{\mathrm{p}}$ His 169; 25, $\mathrm{H} \beta$ ' His169:H $\alpha$ His 169; 26, $\mathrm{NH}_{\mathrm{p}}$ His169:H $\alpha$ His 169; 27, 6-H $\beta: \mathrm{H} \beta$ Arg38. 


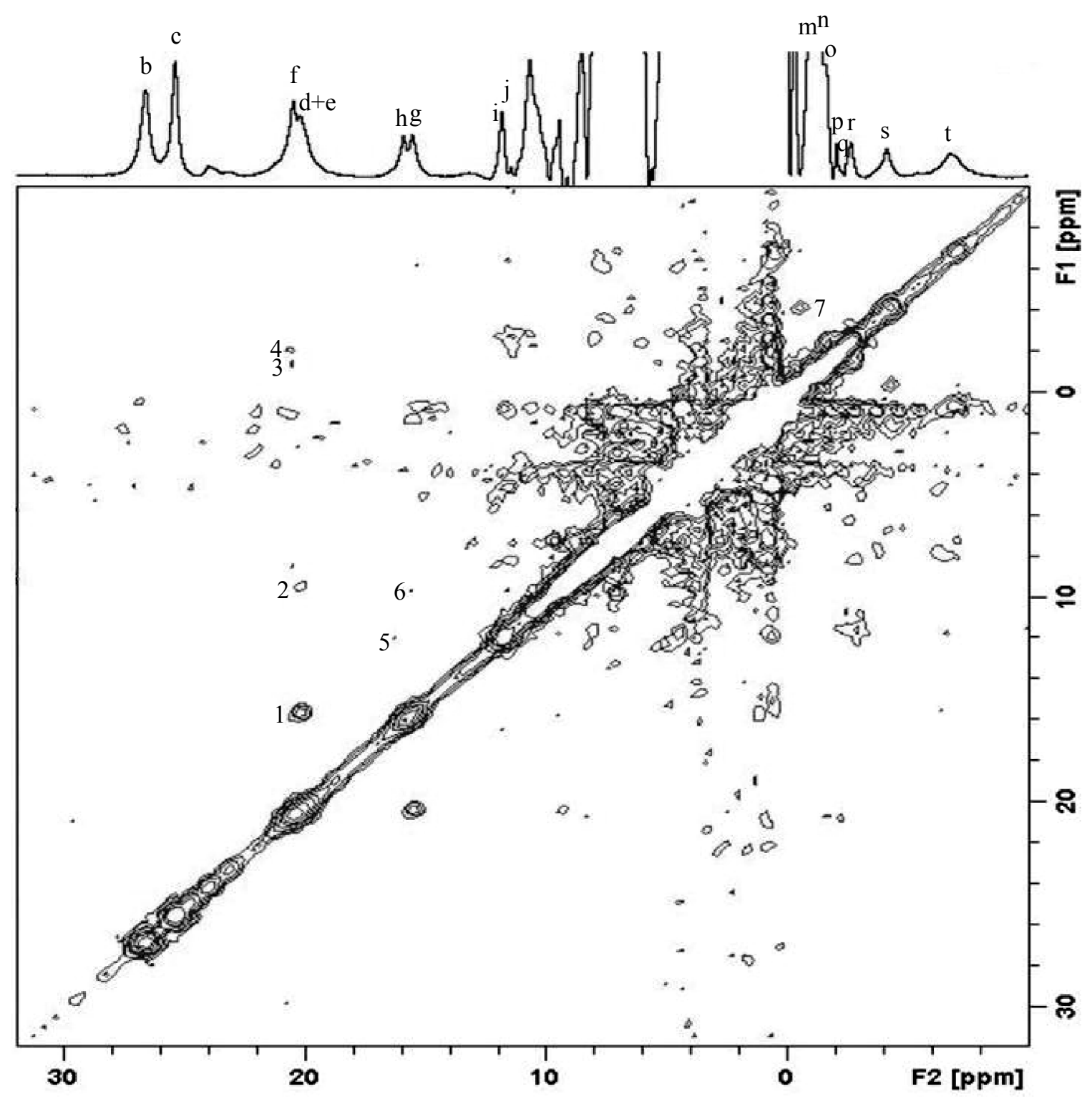

Figure 3.10 The 600-MHz phase-sensitive ${ }^{1} \mathrm{H}$ COSY spectra of $2.5 \mathrm{mM} \mathrm{SBP}^{-\mathrm{CN}^{-}}$in $90 \% \mathrm{H}_{2} \mathrm{O}$ and $10 \%$ $\mathrm{D}_{2} \mathrm{O}$ taken at $323 \mathrm{~K}$. The spectrum is symmetrized for better resolution. Cross-peak assignments in complete agreement with previous 1D and 2D NOE assignments for hyperfine shifted protons are as follows (numbering differently from NOESY spectra): 1, H $\beta$ His169:H $\beta$ ' His 169; 2, H $\beta$ His169:H $\alpha$ His 169;

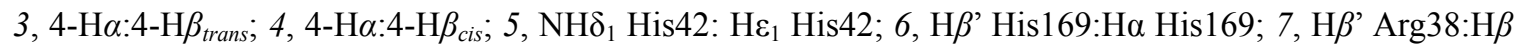
$\operatorname{Arg} 38$. 
Table 3.2 Proton NMR chemical shift ( $\mathrm{ppm}$ ) values of the diamagnetic resonances for the low-spin cyanide derivatives of SBP (assigned through NOESY ${ }^{\mathrm{a}}$ ) and HRP ${ }^{\mathrm{b}}$.

\begin{tabular}{|c|c|c|c|c|c|}
\hline \multirow{2}{*}{\multicolumn{3}{|c|}{$\frac{\text { SBP-CN }}{\text { Shift (ppm) }}$}} & \multirow{2}{*}{\multicolumn{2}{|c|}{$\begin{array}{c}\text { HRP-CN'- } \\
\text { Shift (ppm) }\end{array}$}} & \multirow{3}{*}{ Assignment } \\
\hline & & & & & \\
\hline $308 \mathrm{~K}$ & $313 \mathrm{~K}$ & $323 \mathrm{~K}$ & $308 \mathrm{~K}$ & $328 \mathrm{~K}$ & \\
\hline 0.27 & 0.30 & 0.39 & 0.47 & 0.57 & 7-propionate $\mathrm{H} \beta$ \\
\hline 2.37 & 2.68 & 2.47 & 2.63 & 2.54 & 7-propionate $\mathrm{H} \beta$, \\
\hline--- & 7.01 & 6.95 & 9.65 & 9.74 & 7-propionate $\mathrm{H} \alpha$ \\
\hline 1.90 & --- & 1.62 & --- & 1.90 & $\alpha_{\text {meso }}-\mathrm{H}$ \\
\hline 7.35 & 7.39 & 7.33 & 6.87 & 7.15 & $\beta_{\text {meso }}-\mathrm{H}$ \\
\hline--- & 4.34 & --- & --- & 5.25 & $\gamma_{\text {meso }}-\mathrm{H}$ \\
\hline 6.86 & 6.87 & 7.0 & 6.32 & 6.70 & $\delta_{\text {meso }}-\mathrm{H}$ \\
\hline 10.0 & 9.96 & 9.42 & --- & 9.25 & His169 H $\alpha$ \\
\hline 5.14 & 5.22 & 5.33 & --- & 5.55 & Phe 41 ring $H$ \\
\hline--- & -0.8 & $-0.54^{\mathrm{c}}$ & $-0.61^{\mathrm{d}}$ & -0.6 & Arg38 H $\beta^{\prime}$ \\
\hline--- & 1.7 & $1.47^{\mathrm{c}}$ & $0.93^{d}$ & 0.9 & $\operatorname{Arg} 38 \mathrm{H} \delta^{\prime}$ \\
\hline
\end{tabular}

${ }^{a}$ Results for SBP-CN ${ }^{-}$at $308 \mathrm{~K}$ and $313 \mathrm{~K}$ are obtained in $\mathrm{D}_{2} \mathrm{O}$ buffer; and at $323 \mathrm{~K}$ are obtained in $90 \%$ $\mathrm{H}_{2} \mathrm{O}$ and $10 \% \mathrm{D}_{2} \mathrm{O}$ buffer; note that the isotopic effect may slightly vary the chemical shift values.

${ }^{\mathrm{b}}$ Results for HRP-CN${ }^{-}$are from ref (47-48).

${ }^{\mathrm{c}}$ Observed at different contour level. Data not shown.

${ }^{\mathrm{d}}$ Results are from ref $(50)$ collected at $323 \mathrm{~K}$.

The proton NMR spectra for both the ferric high-spin and low spin cyanide bound forms of SBP are substantially changed when calcium is partially removed from the protein (Figures 3.12). Similar changes were reported for HRP upon calcium removal (54-55). The spectral changes were interpreted as arising from the substantial alterations in the heme environments, most likely the heme proximal and distal sides. Incubation of the calcium-depleted SBP with excess $\mathrm{CaCl}_{2}$ resulted in the re-uptake of calcium as is reflected by the proton NMR spectra of the calcium recombined SBP (data not shown) which are essentially identical to those of the native SBP (Figures 3.1 and 3.2). Similar, reversible behavior of the bound calcium ion in both HRP and SBP implies that the ion plays some common function in the two proteins. 

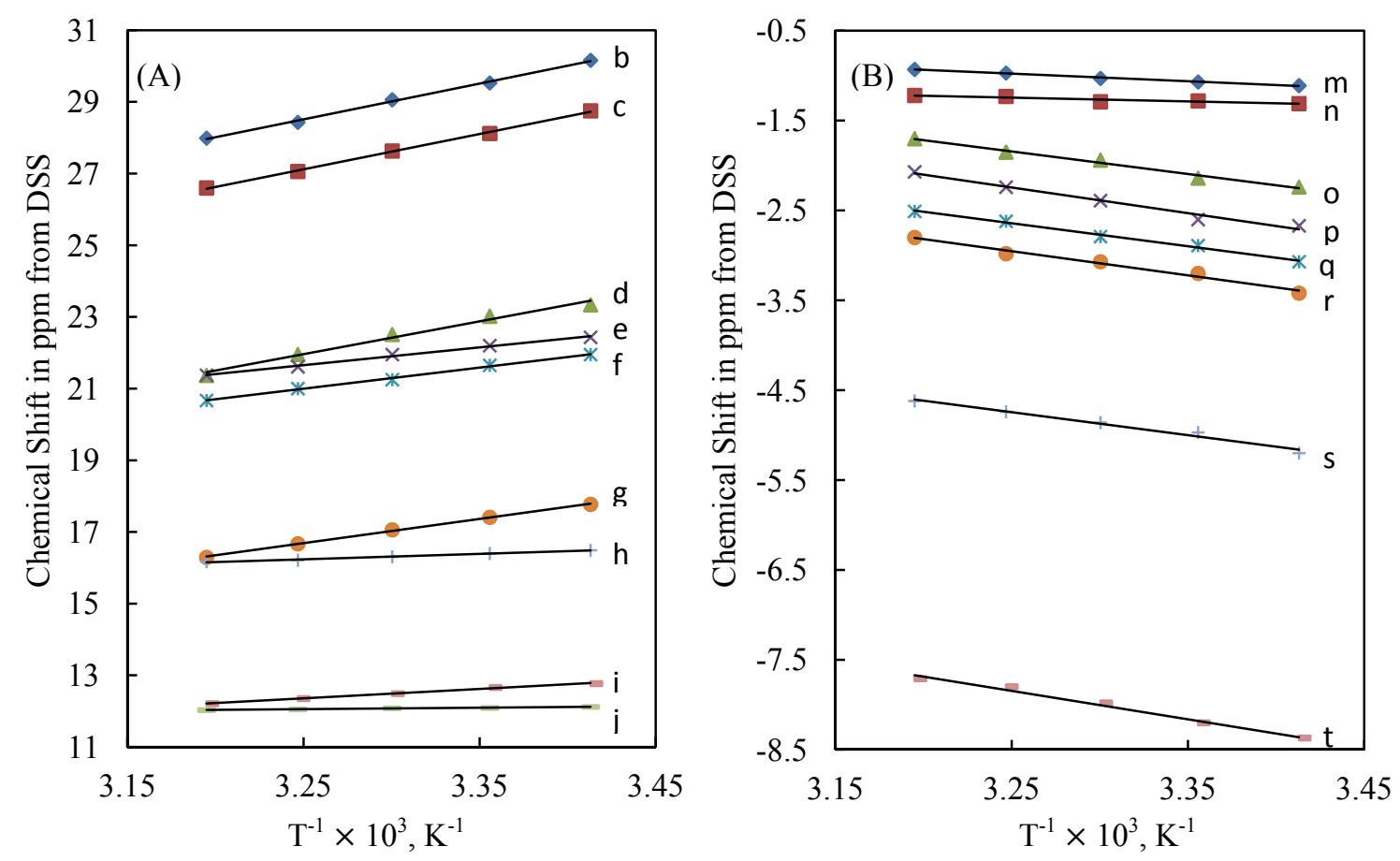

Figure 3.11 Curie plots of observed shift vs. reciprocal temperature for the resolved hyperfine shifted resonances of SBP-CN ${ }^{-}$in the (A) downfield and (B) upfield regions. The letter designation of each peak following the scheme of Figure $3.3 \mathrm{~A}$ is given to the right of each line, and the extrapolated diamagnetic intercept at $\mathrm{T}^{-1}=0$ is given in Table 3.1.

\subsection{Discussion}

\subsubsection{Assignment of the Hyperfine-Shifted Resonances for Native SBP}

Assignment of the paramagnetically-shifted signals for high-spin heme proteins was previously thought to be problematic as the signals are invariable broad and application of NOE and two dimensional NMR methods are thwarted by the extremely short relaxation times of the shifted protons. However, the advances in the NMR technology and methodology combined with isotope labeling has allowed the complete assignment of the hyperfine-shifted signals in several high-spin hemoprotein systems such as HRP, $\mathrm{CcP}$ and lignin peroxidase $(34,44,49,57)$. On the other hand, the peculiar magnetic property of the high-spin form of heme peroxidases also serves as an important hint for 


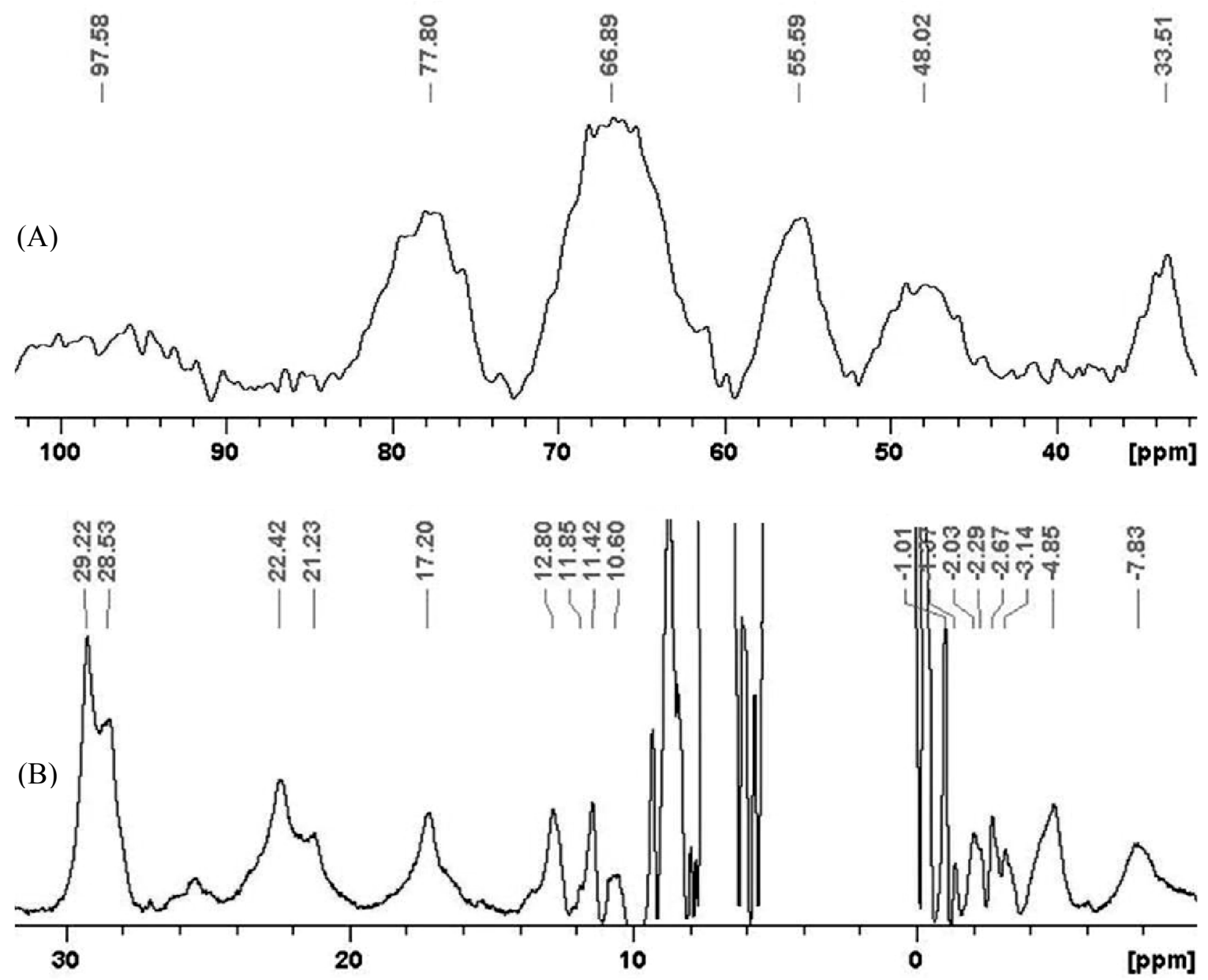

Figure 3.12 The 600-MHz proton NMR spectra of (A) calcium "depleted" ferric high-spin form and (B) calcium "depleted" ferric low-spin cyanide-ligated form of SBP in $10 \mathrm{mM}$ phosphate buffer in $\mathrm{D}_{2} \mathrm{O}$ at $\mathrm{pH}$ $6.8,298 \mathrm{~K}$. Proton chemical shift is referenced with respect to the signal of HDO in the sample solution.

the assignment of the isotropically-shifted signals. For high-spin heme-containing proteins, only protons which directly interact with the heme iron through a net of covalent bonds are expected to exhibit substantial hyperfine shifts, as the magnetic anisotropy is negligible in such systems. By analogy with $\mathrm{HRP}$ and $\mathrm{CcP}$, it is anticipated that only the resonances of the 1-, 3-, 5-, 8- $\mathrm{CH}_{3}$, of the 2- and 4- $\mathrm{H}_{\alpha}$, of the 6- and 7- $\mathrm{H}_{\alpha}$ protons, and of the $\mathrm{H}_{\beta}$ protons of the proximal His (Figure 3.2) will be well-shifted outside the diamagnetic region in the down field region of the spectrum $(44,49,51,58$ - 
59). The chemical shift values for the paramagnetically-shifted signals for native highspin SBP are shown in Table 3.3 with the corresponding results from HRP for comparison.

Table $3.3^{\text {a }}$ Proton NMR chemical shift $(\mathrm{ppm})$ values of the isotropically shifted resonances for the high-spin native SBP and HRP.

\begin{tabular}{cccc}
\hline Signal & SBP & HRP & Assignment $^{\mathrm{b}}$ \\
\hline A & 97.7 & 95.2 & His169 N- $\delta_{1}-\mathrm{H}$ \\
B & 79.3 & 73.7 & $5-\mathrm{CH}_{3}$ \\
C & 69.0 & 67.7 & $1-\mathrm{CH}_{3}$ \\
D & 59.5 & 65.8 & $2-\mathrm{H}_{\alpha}$ \\
E & 55.8 & 64.3 & $8-\mathrm{CH}_{3}$ \\
F & 33.2 & 49.6 & $3-\mathrm{CH}_{3}$ \\
G & 30.1 & 48.3 & $7-\mathrm{H}_{\alpha}$ \\
H & 26.7 & 46.1 & $6-\mathrm{H}_{\alpha}$ \\
\hline
\end{tabular}

${ }^{a}$ Chemical shifts are relative to the residual HDO signal at $4.76 \mathrm{ppm}$. Measurement are carried out at $298 \mathrm{~K}$. Results for HRP are from ref (59) collected at $328 \mathrm{~K}$.

${ }^{\mathrm{b}}$ Assignments are achieved through relative pattern instead of chemical shift value comparison because of the large temperature dependence of the chemical shifts.

\subsubsection{Hyperfine-Shifted Heme Resonances Assignments for the SBP-CN${ }^{-}$Complex}

As was stated in Chapter I, the low-spin nature of the cyanide adducts of heme peroxidases invariably gives well-resolved, sharp signals in their proton NMR spectra, providing a wealth of unique information on the electronic, magnetic, and molecular structural properties of the heme pocket. Therefore, the detection, assignment, and interpretation of the isotropically-shifted signals of the low-spin form peroxidase have been one of the most interesting challenges in NMR investigations. Although 2D NMR methods have been applied to several paramagnetic systems, steady-state 1D NOE 
experiments remain to be the most sensitive techniques for revealing magnetization transfer and are still the preferred techniques for detecting connectivities between very closely spaced signals (60). Therefore, I have performed a series of 1D NOE experiments, in order to assign the hyperfine-shifted signals for the SBP- $\mathrm{CN}^{-}$complex as are shown in Figures 3.4(B to E), and Figures 3.5(B to F).

Because of the relatively longer relaxation time and closer position of the hyperfineshifted resonances in SBP-CN${ }^{-}$than those in $\mathrm{HRP}_{-} \mathrm{CN}^{-}$, particular care must be paid when setting up the NOE experiment to prevent power spillage and off-resonance effects which could mask the real NOE effect. As is shown in Figures 3.4B and 3.4C, irradiation of the two heme methyl resonances "b" and "c" gives NOE patterns that can be attributed to a methyl group near either a vinyl or propionate group (Figure 1.1B). The NOE experiments on HRP-CN${ }^{-}$have shown that the heme methyls which are close to a vinyl group exhibit NOEs to either the $\alpha-\mathrm{CH}$ proton or the $\beta-\mathrm{CH}_{2}$ protons depending on the vinyl group orientation as shown in Figure 1.1B (47-48). The $\alpha-\mathrm{CH}$ vinyl proton resonance is normally observed in the down field region because of $\pi$-spin delocalization of the unpaired spin density in the vinyl group, whereas the $\beta-\mathrm{CH}_{2}$ vinyl proton peaks are usually positioned between 0 to $-5.0 \mathrm{ppm}$. Saturation of peak "b" yields detection of peak "f" at "20.7" ppm and peak " $n$ " at "-1.2" ppm in the difference spectrum. Several other peaks are also observed in the difference spectrum in the diamagnetic aliphatic region, indicating the development of multiple secondary NOEs or spin diffusion (61). The lack of selectivity in NOEs is a result of the relatively longer irradiation times used in setting up the experiment (48). Nevertheless, observation of the NOE to the peak at "20.7" ppm correspond to the result observed for $\mathrm{HRP}-\mathrm{CN}^{-}$when the $8-\mathrm{CH}_{3}$ signal is irradiated (48). 
Comparison of the spectral pattern of $\mathrm{SBP}-\mathrm{CN}^{-}$with that of $\mathrm{HRP}^{-\mathrm{CN}^{-}}$leads to the assignment of resonance " $b$ " to the 5 - or $8-\mathrm{CH}_{3}$ group $(34,45,48)$.

The detection of only two of the four heme methyl proton signals outside the diamagnetic region is consistent with earlier observations for $\mathrm{HRP}_{-} \mathrm{CN}^{-}$and other lowspin hemoprotein cyanide complexes. The result has been attributed to the particular orientation of the proximal histidine ring with respect to the heme plane $(48,62)$. Depending on the position of the proximal histidine, the four heme methyls display a unique isotropic shift pattern as either the $1-\mathrm{CH}_{3}$ and $5-\mathrm{CH}_{3}$ or the $3-\mathrm{CH}_{3}$ and $8-\mathrm{CH}_{3}$ pair in the down field region (62-63). Therefore, the methyl peak "c" at $26.6 \mathrm{ppm}$ must arise from either the $1-\mathrm{CH}_{3}$ or the $3-\mathrm{CH}_{3}$ group of the heme unit. The observed resonance "c" for $\mathrm{SBP}-\mathrm{CN}^{-}$is assigned to the $3-\mathrm{CH}_{3}$ group, as irradiation of peak "c" gives $\mathrm{NOE}$ patterns very similar to those reported for $\mathrm{HRP}$ when $3-\mathrm{CH}_{3}$ is irradiated (48). By analogy with HRP-CN" (48), the peaks "e" at 21.4 ppm observed in the NOE difference spectrum by saturation peak "c" (Figure 3.4C) can be assigned to $4-\mathrm{H}_{\alpha}$, and the four upfield shifted peaks " $m$ " at -0.9 , "o" at -1.7 , "p" at -2.1 , and " $\mathrm{r}$ " at $-2.8 \mathrm{ppm}$ should arise from all the four vinyl $\beta$-protons of heme. Since peak "o" displays the strongest NOE correlation with the $3-\mathrm{CH}_{3}$ group, it can be assigned to the $4-\mathrm{H}_{\beta}-\mathrm{t}$, which is the most proximate proton to the $3-\mathrm{CH}_{3}$ group (Figure 1.1B). Saturation of the assigned $4-\mathrm{H}_{\alpha}$ (peak "e") yields the difference trace of Figure 3.4D, in which peak "o" and "p" exhibit clear NOE connections with the 4- $\mathrm{H}_{\alpha}$, therefore peaks "p" which has stronger NOE with peak "e" must arise from the other $4-\mathrm{H}_{\beta}$ proton $\left(4-\mathrm{H}_{\beta}-\mathrm{c}\right)$. Consequently, the two remaining peaks " $m$ " and " $\mathrm{r}$ " can be assigned to the $2-\mathrm{H}_{\beta}-\mathrm{c}$ and $2-\mathrm{H}_{\beta}-\mathrm{t}$, respectively.

The spectral pattern of the four vinyl $\mathrm{H}_{\beta}$ 's between SBP and HRP is slightly different, 
indicating the existence of minor heme cavity discrepancy in these two peroxidases. The absence of an $\mathrm{NOE} 4-\mathrm{H}_{\alpha} \rightarrow 3-\mathrm{CH}_{3}$ (Figure 3.4D) dictate that the 4-vinyl group is close to in-plane and is oriented in the cis position $(48,58)$. Moreover, upon saturation of 3- $\mathrm{CH}_{3}$, the relative magnitudes of the NOEs, $4-\mathrm{H}_{\beta-\mathrm{t}}>4-\mathrm{H}_{\beta-\mathrm{c}} \approx 2-\mathrm{H}_{\beta-\mathrm{t}}>2-\mathrm{H}_{\beta-\mathrm{c}}$ are qualitatively consistent with distances estimated for trans-2-vinyl and cis-4-vinyl, therefore unambiguously establishes the orientations of the 2- and 4-vinyl groups in SBP-CN.

While peak "c" is now unequivocally assigned to the $3-\mathrm{CH}_{3}$, peak "b" at $28.0 \mathrm{ppm}$ must arise from the $8-\mathrm{CH}_{3}$ and the NOE observed for the peak "f" at $20.7 \mathrm{ppm}$ upon saturation of peak "b" must arise from a 7-propionate $\mathrm{H}_{\alpha}$. The assignment of peak " $\mathrm{n}$ " at "- 1.2 " ppm is circuitous, because the equivalent peak of HRP was originally assigned to a leucine methyl group (49) and later on revealed through 2D NMR methods as deriving from an Ile244 methyl, which is in proximity to the $8-\mathrm{CH}_{3}(56)$. However, in SBP, the corresponding Ile position is replaced with Leu243, whose methyl group is only $4.13 \AA$ from the $8-\mathrm{CH}_{3}$ (carbon-carbon distance, measured from the rSBP crystal structure). Therefore, peak " $n$ " is assigned to the Leu243 $\delta_{2}-\mathrm{CH}_{3}$.

Figure 3.5F shows the NOE difference spectra obtained upon saturating a far upfield shifted peak "q" at $-2.5 \mathrm{ppm}$. The absence of NOE with any assigned hyperfine heme resonances indicates that it is not in proximity to 2-, 3-, 4-, 7-, or 8-substituents of the heme. The ${ }^{1} \mathrm{H}$ NMR results of HRP-CN ${ }^{-}$complex (48) reveals that the resonance assigned to the 6-propionate $\mathrm{H}_{\beta}$ is almost identical in both chemical shift value $(-2.8 \mathrm{ppm})$ and temperature dependence (Curie plot intercept at $5.4 \mathrm{ppm}$ in comparison with $5.7 \mathrm{ppm}$ for peak "q"), and additionally exhibits similar NOE pattern to peak "q", leading the final assignment of peak "q" to one of the $\beta$ protons of 6-propionate group. 


\subsubsection{Distal and Proximal Histidine Assignments for the SBP-CN ${ }^{-}$Complex}

The appearance of the exchangeable peak "a" at $26.7 \mathrm{ppm}$ is an indication of the presence of a distal histidine (His42) within the heme pocket of SBP, which is consistent with the rSBP crystal structure. This exchangeable proton has been detected for a number of heme peroxidases, such as $\mathrm{HRP}, \mathrm{CcP}$, and $\operatorname{LiP}(34,47-48)$. Support for the existence of the distal histidine is also found in the presence of another labile proton signal " $\mathrm{h}$ " at 16.2 ppm which is comparable to the position of 16.3 and $16.5 \mathrm{ppm}$ for the corresponding proton in $\mathrm{HRP}-\mathrm{CN}^{-}$and $\mathrm{C} c \mathrm{P}-\mathrm{CN}^{-}(34)$. No attempt through 1D NOE experiments was made to identify NOE connectivities of the labile protons to and from other signals. The assignment of peak " $h$ " to the His $42 \mathrm{~N}-\delta_{1}-\mathrm{H}$ is somewhat arbitrary, but is confirmed through 2D NOESY experiment as will be later discussed. The absence of NOE to hyperfine peaks when peak "i" at $12.2 \mathrm{ppm}$ is irradiated (Figure 3.5C) suggested that peak "i" is not in contact with any of other hyperfine-shifted signals. The corresponding peak at $13.3 \mathrm{ppm}$ with similar temperature dependence (Curie plot intercept at $3.6 \mathrm{ppm}$ in comparison with 3.8 ppm for peak "ii") in HRP has been assigned to the C- $\varepsilon_{1}-\mathrm{H}$ of the distal histidine (34). By analogy, peak "i”" for SBP can also be assigned to the C- $\varepsilon_{1}-\mathrm{H}$ of the distal histidine (His42).

The presence of the extremely broad, fast relaxing, far upfield-shifted peak " $\mathrm{z}$ " at $-28.3 \mathrm{ppm}$ is a characteristic feature of the existence of the proximal histidine within the heme crevice. This peak has been previously detected for the low-spin cyanide adducts of a number of heme peroxidases, such as HRP, CcP, Lip, LPO, and MPO, and is assigned to the C-E1-H of the proximal histidine imidazole ring $(34,50,56,64-65)$. The line width and chemical shift values of peak " $\mathrm{z}$ " in $\mathrm{SBP}-\mathrm{CN}^{-}$match quit closely to those of the 
corresponding peak in $\mathrm{HRP}-\mathrm{CN}^{-}$, suggesting the imidazolate character of the proximal histidine for $\mathrm{SBP}-\mathrm{CN}^{-}$as was earlier determined for $\mathrm{HRP}_{-} \mathrm{CN}^{-}(50,65)$. Additional support for the presence of the proximal histidine is found in the observation of another broad, very fast relaxing signal " $\mathrm{y}$ " at $30.1 \mathrm{ppm}$ which has been assigned to the C- $\varepsilon 2-\mathrm{H}$ of the proximal histidine in several heme peroxidases (45).

Signals "d" and "g" are assigned to the C- $\beta-H s$ of the proximal histidine (His169), as an intense NOE is observed for resonance "g" at $16.3 \mathrm{ppm}$ when peak "d" at $21.4 \mathrm{ppm}$ is irradiated (Figure 3.4D). Support for this assignment is found from the strong reciprocal NOE to peak "d" when signal "g" is saturated (Figure 3.5B), confirming the geminal nature of peak "d" and "g". Saturation of peak "d" (Figure 3.4D) and "g" (Figure 3.5B) both yields NOEs to peak "i" and several common peaks in the diamagnetic region. Integration of peak "d" reveals that two protons are overlapped at the same position at all temperatures in $\mathrm{SBP}-\mathrm{CN}^{-}$, which is the same as in $\mathrm{HRP}-\mathrm{CN}^{-}$. Correspondingly the other proton at $21.4 \mathrm{ppm}$ is assigned to the His $169 \mathrm{C}-\delta_{2}-\mathrm{H}$.

Another unfortunate two-proton overlap occurs at peak "i" at $12.2 \mathrm{ppm}$, which shows weak or strong NOE correlation upon irradiation of peak "d" or "g", respectively. However, as mentioned above, no reciprocal NOE is observed while saturating peak " $i$ ", which has been assigned as the distal His $42 \mathrm{C}-\varepsilon_{1}-\mathrm{H}$. Therefore the other proton must arise from one of the proximal His 170 protons. The corresponding peak in HRP at $11.98 \mathrm{ppm}$, which is better resolved without overlapping, is assigned to the proximal histidine $\mathrm{N}_{\text {pep }} \mathrm{H}$ through 2D NMR methods. Therefore, the proton overlaps with peak "i" is tentatively assigned to the His $169 \mathrm{~N}_{\text {pep }} \mathrm{H}$, which is resistant to isotope exchange in holoprotein, and waiting to be proved by 2D NMR experiments. 
The two labile protons " $h$ " and "j" are assigned through comparison with HRP and $\mathrm{C} c \mathrm{P}$ cyanide complexes to the $\mathrm{N}_{\delta 1}$ protons of the distal and proximal histidine, respectively. The assignment of peak " $\mathrm{h}$ " is relatively straightforward because of the similar chemical shift position as comparing $\mathrm{HRP}$ and $\mathrm{CcP}$ and has been proved by NOESY experiment as shown later. However, the peak “ $\mathrm{j}$ ” was originally assigned to the $\mathrm{N}_{\text {pep }} \mathrm{H}$ of the proximal histidine by solely comparing the chemical shift value. As just mentioned above, the $\mathrm{N}_{\text {pep }} \mathrm{H}$ is resistant to isotope exchange with exchange lifetimes of several years $(66)$, which is in contrast with the rapid exchangeable behavior of proton “ $\mathrm{j}$ ". Therefore, the proton " $\mathrm{j}$ " is assigned to the most possible candidate, which is the His $169 \mathrm{~N}_{\delta 1} \mathrm{H}$ who has a matching Curie plot extrapolated intercept at infinite temperature with “j”.

Although more NOE correlations with proximal histidine protons in the diamagnetic region are revealed through $1 \mathrm{D}$ NOE experiments, no further attempt was made because of the relatively poor resolution within the complicated diamagnetic envelope. More confident assignment for these correlations can only be achieved through 2D NMR.

\subsubsection{Distal Arginine Assignments for the SBP-CN${ }^{-}$Complex}

The presence of a distal Arg as catalytically critical residue was suggested to be highly conserved in HRP (47), CcP (30), and other plant heme peroxidases (67). The rSBP crystal structure also revealed a distal Arg38 located right above the prosthetic heme, with its extended, essentially planar trimethylene side chain extends over pyrrole III. The position and $\mathrm{T}_{1}$ of two upfield hyperfine shifted signals " $\mathrm{s}$ " at $-4.6 \mathrm{ppm}$ and " $\mathrm{t}$ " at $-7.7 \mathrm{ppm}$ are well comparable with resonances of -4.75 and $-6.45 \mathrm{ppm}$ from distal Arg of HRP-CN" (47). Therefore, signals "s" and " $\mathrm{t}$ " are assigned to Arg38 C- $\beta_{1}-\mathrm{H}$ and Arg38 C- 
$\delta_{1}-\mathrm{H}$, correspondingly.

Both the result from HRP and the crystal structure of rSBP suggest that the trimethylene side chain extends over pyrrole III of the prosthetic heme, making contact with the heme 5- $\mathrm{CH}_{3}, 6-\beta-\mathrm{CH}_{2}$, and $\gamma$-meso-H. Although resonances from the heme 5$\mathrm{CH}_{3}$, and $\gamma$-meso-H are not assigned through 1D NOE methods in this study, irradiations of peak "s" and " $\mathrm{t}$ " (Figure 3.5D and E) both yield a clear NOE to peak "q" at -2.5 ppm, which has been formerly assigned to 6-propionate $\mathrm{H}_{\beta}$. Reciprocal NOE correlation between peak "q", "s" and "t" (Figure 3.5F) indicating that Arg38 side chain does extend above pyrrole III, in complete agreement with the rSBP crystal structure.

\subsubsection{D NOESY and COSY Results for the SBP-CN'Complex}

The NOESY experiments were carried out at different conditions: $308 \mathrm{~K}$ (Figure 3.7), and $313 \mathrm{~K}$ (Figure 3.8) in $\mathrm{D}_{2} \mathrm{O}$, and $323 \mathrm{~K}$ (Figure 3.9) in $90 \% \mathrm{H}_{2} \mathrm{O}$ and $10 \% \mathrm{D}_{2} \mathrm{O}$, to minimize the affect of overlaps and to verify previous nonexchangeable and labile proton assignments. Most of the 1D NOE correlations are confirmed in NOESY specta, especially for correlations between the hyperfine shifts. The assignments of the labile proton peaks "h" and "j", together with the overlapping peaks "d" and "i", are all confirmed as shown in Figure 3.9. Moreover, resonances which have been buried in the diamagnetic envelop in the 1D NMR results are now exposed with high resolutions and allow further assignments for the SBP-CN ${ }^{-}$complex by comparison with $\mathrm{HRP}^{-\mathrm{CN}^{-}} \mathrm{NMR}$ assignments. The assignments and corresponding chemical shift values at different conditions are shown in Table 3.2. All the four meso-protons and the 7-propionate protons of the prosthetic heme, together with the proximal His $170 \mathrm{H} \alpha$ and two distal Arg38 protons are successfully assigned through 2D NMR. The COSY spectrum in $90 \% \mathrm{H}_{2} \mathrm{O}$ 
and $10 \% \mathrm{D}_{2} \mathrm{O}$ further confirmed the assignments by revealing the spin-spin coupling correlations between proximal and distal histidine protons, 4-vinyl protons and distal arginine protons (Figure 3.10).

In summary, 16 of all the 22 resonances of the prosthetic heme, all proximal His170 resonances, and all $\beta$ and $\delta$ proton resonances of the distal $\operatorname{Arg} 38$ in $\mathrm{SBP}_{-} \mathrm{CN}^{-}$complex are identified through the combination of 1D and 2D NOE NMR methods in this work. All hyperfine-shifted signals are unambiguously assigned. Additionally, the cis-, transorientation of the two vinyl groups and the orientation of the distal arginine are confirmed as consistent with the recombinant SBP crystal structure.

\subsubsection{Attempted Calcium Removal and Reconstitution}

Partial removal of calcium from SBP causes substantial changes in the proton NMR spectral features of both the ferric high-spin and cyanide adduct low-spin forms of the protein (Figures 3.12). For the native enzyme, the four heme peripheral methyl proton signals are observed at 79.3, 69.0, 59.5, and $55.8 \mathrm{ppm}$, while the signals for the $\mathrm{Ca}^{2+}$ depleted enzyme are located at $77.8,66.9,55.6$, and $48.0 \mathrm{ppm}$. The heme methyl proton peaks of the low-spin cyanide complex of the $\mathrm{Ca}^{2+}$-depleted SBP are observed at 29.2 and $28.5 \mathrm{ppm}$, whereas those of the cyanide complex of the native SBP are located at 29.5 and $28.1 \mathrm{ppm}$. More minor spectral differences are also noticeable between these spectra. The spectral changes derived from the calcium removal of SBP are similar as reported for HRP (54-55), indicating that calcium ions play the same structural and functional roles in these two peroxidases.

Similar as in HRP, addition of an excess amount of $\mathrm{CaCl}_{2}$ to the calcium "depleted" SBP regenerates the spectral changes caused upon calcium removal (data not shown), 
indicating the reversible binding of calcium within the protein.

The spin state of the heme iron of the $\mathrm{Ca}^{2+}$-depleted HRP is reported to be much different from that of the heme iron of the native protein (a thermal spin mixing between ferric high and low spin states vs. pure ferric high spin state) as is reflected in the dramatic changes of the chemical shift values of the two forms of the protein (54-55). It is also reported recently that the $\mathrm{Ca}^{2+}$ depletion of HRP does not change the alpha helicity of the protein, but it changes the whole tertiary structure to a loosened, molten globulelike conformation, introduces tertiary structural changes in the immediate vicinity of the heme, and also increase the overall planarity of the heme (68). The high similarity of spectral changes caused by calcium depletion for SBP and HRP suggests that calcium ions play same role in these two closed related enzymes.

\subsubsection{Comparison between SBP and HRP}

Proton NMR examination of both the high-spin native and the low-spin cyanide ligated forms of SBP suggests the presence of the same prosthetic group, iron protoporphyrin IX, in this protein. Confirmation of this comes from the identical NMR spectral pattern of the extracted prosthetic group from SBP to that of the standard hemin. NMR spectral analysis reveals proximal histidine ligation to the heme iron and the presence of a distal histidine in the active site vicinity. Moreover, the obvious similarity between the proton NMR hyperfine shifts of SBP-CN ${ }^{-}$and $\mathrm{HRP}-\mathrm{CN}^{-}$indicates that the two enzymes adopt the same heme geometry. The similar structural features of the active cavity of SBP and HRP suggest similar reactivates of these two plant peroxidases, as is reflected by their similar substrate profile. The trans and cis orientations of the 2- and 4vinyl groups of the heme in SBP and HRP are the same and therefore suggesting identical 
heme orientations.

However, differences do exist between SBP and HRP. Major differences include the chemical shift and $T_{1}$ values of the two hyperfine-shifted methyl signals and the proton resonance positions of both the proximal and distal histidine residues as were shown in Figures 3.3 and Table 3.1. Additionally, the 2 - and 4- vinyl $\beta$ protons corresponding position are slightly different in $\operatorname{SBP}\left(2-\mathrm{H}_{\beta-c}>4-\mathrm{H}_{\beta-\mathrm{t}}>4-\mathrm{H}_{\beta-\mathrm{c}}>2-\mathrm{H}_{\beta-\mathrm{t}}\right)$ and $\mathrm{HRP}\left(2-\mathrm{H}_{\beta-\mathrm{c}}>\right.$ 4- $\left.\mathrm{H}_{\beta-\mathrm{t}}>2-\mathrm{H}_{\beta-\mathrm{t}}>4-\mathrm{H}_{\beta-\mathrm{c}}\right)$. These dissimilarities can be attributed to the differences in amino acid sequence and/or tertiary structures of the two proteins. The difference of the iron out-of-planarity or heme ruffling between these two closely related peroxidases may also affect their NMR spectral properties significantly.

\subsection{References}

1. Ramos, M. C., Torijas, M. C., and Díaz, A. N. (2001) Enhanced chemiluminescence biosensor for the determination of phenolic compounds and hydrogen peroxide, Sensors and Actuators B: Chemical 73, 71-75.

2. Iwuoha, E. I., Joseph, S., Zhang, Z., Smyth, M. R., Fuhr, U., and Ortiz de Montellano, P. R. (1998) Drug metabolism biosensors: electrochemical reactivities of cytochrome P450cam immobilised in synthetic vesicular systems, Journal of Pharmaceutical and Biomedical Analysis 17, 1101-1110.

3. Yoshida, Kaothien, Matsui, Kawaoka, and Shinmyo. (2003) Molecular biology and application of plant peroxidase genes, Applied Microbiology and Biotechnology 60, 665-670.

4. Velde, F. v. d., Rantwijk, F. v., and Sheldon, R. A. (2001) Improving the catalytic performance of peroxidases in organic synthesis, Trends in Biotechnology 19, 73-80.

5. Veitch, N. C. (2004) Horseradish peroxidase: a modern view of a classic enzyme, Phytochemistry 65, 249-259.

6. Henriksen, A., Mirza, O., Indiani, C., Teilum, K., Smulevich, G., Welinder, K. G., and Gajhede, M. (2001) Structure of soybean seed coat peroxidase: A plant peroxidase with unusual stability and haem-apoprotein interactions, Protein Science 10, 108-115. 
7. Pokora, A. R., and Johnson, M. A. (1993) Soybean peroxidase treatment of contaminated substances, The Mead Corporation (Dayton, OH), United States.

8. McEldoon, J. P., and Dordick, J. S. (1996) Unusual Thermal Stability of Soybean Peroxidase, Biotechnology Progress 12, 555-558.

9. Kamal, J. K. A., and Behere, D. V. (2002) Thermal and Conformational Stability of Seed Coat Soybean Peroxidase, Biochemistry 41, 9034-9042.

10. Kamal, J. K. A., and Behere, D. V. (2003) Activity, stability and conformational flexibility of seed coat soybean peroxidase, Journal of Inorganic Biochemistry 94, 236242.

11. Kamal, J. K. A., and Behere, D. V. (2008) Kinetic stabilities of soybean and horseradish peroxidases, Biochemical Engineering Journal 38, 110-114.

12. Patapas, J., Al-Ansari, M. M., Taylor, K. E., Bewtra, J. K., and Biswas, N. (2007) Removal of dinitrotoluenes from water via reduction with iron and peroxidase-catalyzed oxidative polymerization: A comparison between Arthromyces ramosus peroxidase and soybean peroxidase, Chemosphere 67, 1485-1491.

13. Gómez, J. L., Bódalo, A., Gómez, E., Hidalgo, A. M., Gómez, M., and Murcia, M. D. (2008) A transient design model of a continuous tank reactor for removing phenol with immobilized soybean peroxidase and hydrogen peroxide, Chemical Engineering Journal $145,142-148$.

14. Al-Ansari, M. M., Steevensz, A., Al-Aasm, N., Taylor, K. E., Bewtra, J. K., and Biswas, N. (2009) Soybean peroxidase-catalyzed removal of phenylenediamines and benzenediols from water, Enzyme and Microbial Technology 45, 253-260.

15. Gomez, M., Matafonova, G., Gomez, J. L., Batoev, V., and Christofi, N. (2009) Comparison of alternative treatments for 4-chlorophenol removal from aqueous solutions: Use of free and immobilized soybean peroxidase and $\mathrm{KrCl}$ excilamp, Journal of Hazardous Materials 169, 46-51.

16. Jiménez, L., Parra, M., Tomás, V., Martinez-Gutiérrez, R., García-Cánovas, F., and Tudela, J. (2009) Indigo carmine biodegradation catalysed by soybean peroxidase, New Biotechnology 25, S161-S161.

17. Parra, M., Martínez-Ruíz, J., Tomás, V., Martínez-Gutiérrez, R., García-Cánovas, F., and Tudela, J. (2009) Optimization of the soybean peroxidase catalysed biodegradation of 2,4,6-trichlorophenol, New Biotechnology 25, S160-S161.

18. Shi, L., Liu, X., Niu, W., Li, H., Han, S., Chen, J., and Xu, G. (2009) Hydrogen peroxide biosensor based on direct electrochemistry of soybean peroxidase immobilized on single-walled carbon nanohorn modified electrode, Biosensors and Bioelectronics 24, 1159-1163. 
19. Vdovenko, M. M., Ciana, L. D., and Sakharov, I. Y. (2009) 3-(10'Phenothiazinyl)propane-1-sulfonate is a potent enhancer of soybean peroxidase-induced chemiluminescence, Analytical Biochemistry 392, 54-58.

20. Vdovenko, M. M., Zubkov, A. V., Kuznetsova, G. I., Ciana, L. D., Kuzmina, N. S., and Sakharov, I. Y. (2010) Development of ultra-sensitive soybean peroxidase-based CLELISA for the determination of human thyroglobulin, Journal of Immunological Methods $362,127-130$.

21. Marchis, T., Avetta, P., Bianco-Prevot, A., Fabbri, D., Viscardi, G., and Laurenti, E. (2011) Oxidative degradation of Remazol Turquoise Blue G 133 by soybean peroxidase, Journal of Inorganic Biochemistry 105, 321-327.

22. Azevedo, A. M., Martins, V. C., Prazeres, D. M. F., Vojinovic, V., Cabral, J. M. S., and Fonseca, L. P. (2003) Horseradish peroxidase: a valuable tool in biotechnology, In Biotechnology Annual Review (El-Gewely, M. R., Ed.), pp 199-247, Elsevier.

23. Hoyle, M. C. (1977) High Resolution of Peroxidase-Indoleacetic Acid Oxidase Isoenzymes from Horseradish by Isoelectric Focusing, Plant Physiol. 60, 787-793.

24. Bedard, P., and Mabrouk, P. A. (1997) Resonance Raman Spectroscopy of Soybean Peroxidase, Biochemical and Biophysical Research Communications 240, 65-67.

25. Amisha Kamal, J. K., and Behere, D. V. (2001) Steady-State and Picosecond Time-Resolved Fluorescence Studies on Native and Apo Seed Coat Soybean Peroxidase, Biochemical and Biophysical Research Communications 289, 427-433.

26. Welinder, K. G., and Larsen, Y. B. (2004) Covalent structure of soybean seed coat peroxidase, Biochimica et Biophysica Acta (BBA) - Proteins \& Proteomics 1698, 121-126.

27. Sakurada, J., Takahashi, S., and Hosoya, T. (1987) Proton nuclear magnetic resonance studies on the iodide binding by horseradish peroxidase, Journal of Biological Chemistry 262, 4007-4010.

28. de Ropp, J. S., Chen, Z., and La Mar, G. N. (1995) Identification of Residues in the Aromatic Substrate Binding Site of Horseradish Peroxidase by 1H NMR Studies on Isoenzymes, Biochemistry 34, 13477-13484.

29. Poulos, T. L., and Kraut, J. (1980) The stereochemistry of peroxidase catalysis, Journal of Biological Chemistry 255, 8199-8205.

30. Finzel, B. C., Poulos, T. L., and Kraut, J. (1984) Crystal structure of yeast cytochrome c peroxidase refined at 1.7-A resolution, Journal of Biological Chemistry 259, 13027-13036.

31. Montellano, P. R. O. (1992) Catalytic Sites of Hemoprotein Peroxidases, Annual Review of Pharmacology and Toxicology 32, 89-107. 
32. Takio, K., Titani, K., Ericsson, L. H., and Yonetani, T. (1980) Primary structure of yeast cytochrome c peroxidase, II. The complete amino acid sequence, Archives of Biochemistry and Biophysics 203, 615-629.

33. Bertini, I., Turano, P., and Vila, A. J. (1993) Nuclear magnetic resonance of paramagnetic metalloproteins, Chemical Reviews 93, 2833-2932.

34. Banci, L., Bertini, I., Turano, P., Ferrer, J. C., and Mauk, A. G. (1991) Comparative proton NMR study of ferric low-spin cytochrome c peroxidase and horseradish peroxidase, Inorganic Chemistry 30, 4510-4516.

35. Chang, C. S., Yamazaki, I., Sinclair, R., Khalid, S., and Powers, L. (1993) pH dependence of the active site of horseradish peroxidase compound II, Biochemistry 32, 923-928.

36. Pütter, J., and Becker, R. (1983) Methods of Enzymatic Analysis, (Bergmeyer, H. U., Ed.), pp 286-293, Verlag Chemie GmbH, Weinheim.

37. Nelson, D. P., and Kiesow, L. A. (1972) Enthalpy of decomposition of hydrogen peroxide by catalase at $25^{\circ} \mathrm{C}$ (with molar extinction coefficients of $\mathrm{H}_{2} \mathrm{O}_{2}$ solutions in the UV), Analytical Biochemistry 49, 474-478.

38. Maehly, A. C., and Chance, B. (1954) Assay of catalases and peroxidases, In Methods of Biochemical Analysis (Glick, D., Ed.), pp 357-424, Interscience Publishers, New York.

39. Haschke, R. H., and Friedhoff, J. M. (1978) Calcium-related properties of horseradish peroxidase, Biochemical and Biophysical Research Communications 80, 1039-1042.

40. Yonetani, T., and Schleyer, H. (1967) Studies on Cytochrome c Peroxidase, Journal of Biological Chemistry 242, 1974-1979.

41. Hartel, A. J., Lankhorst, P. P., and Altona, C. (1982) Thermodynamics of Stacking and of Self-Association of the Dinucleoside Monophosphate m62A-U from Proton NMR Chemical Shifts, European Journal of Biochemistry 129, 343-357.

42. Noggle, J. H., and Schirmer, R. E. (1971) The nuclear Overhauser effect: chemical applications, Academic Press, New York.

43. La Mar, G. N., and de Ropp, J. S. (1979) Assignment of exchangeable proximal histidine resonances in high-spin ferric hemoproteins: Substrate binding in horseradish peroxidase, Biochemical and Biophysical Research Communications 90, 36-41.

44. Lukat, G. S., Rodgers, K. R., Jabro, M. N., and Goff, H. M. (1989) Magnetic resonance spectral characterization of the heme active site of Coprinus cinereus peroxidase, Biochemistry 28, 3338-3345. 
45. Banci, L., Bertini, I., Pease, E. A., Tien, M., and Turano, P. (1992) Proton NMR investigation of manganese peroxidase from Phanerochaete chrysosporium. A comparison with other peroxidases, Biochemistry 31, 10009-10017.

46. De Ropp, J. S., La Mar, G. N., Smith, K. M., and Langry, K. C. (1984) Proton NMR studies of the electronic and molecular structure of ferric low-spin horseradish peroxidase complexes, Journal of the American Chemical Society 106, 4438-4444.

47. Thanabal, V., De Ropp, J. S., and La Mar, G. N. (1987) Identification of the catalytically important amino acid residue resonances in ferric low-spin horseradish peroxidase with nuclear Overhauser effect measurements, Journal of the American Chemical Society 109, 7516-7525.

48. Thanabal, V., DeRopp, J. S., and La Mar, G. N. (1987) Proton NMR study of the electronic and molecular structure of the heme cavity in horseradish peroxidase. Complete heme resonance assignments based on saturation transfer and nuclear Overhauser effects, Journal of the American Chemical Society 109, 265-272.

49. Thanabal, V., De Ropp, J. S., and La Mar, G. N. (1988) Proton NMR characterization of the catalytically relevant proximal and distal hydrogen-bonding networks in ligated resting state horseradish peroxidase, Journal of the American Chemical Society 110, 3027-3035.

50. La Mar, G. N., De Ropp, J. S., Chacko, V. P., Satterlee, J. D., and Erman, J. E. (1982) Axial histidyl imidazole non-exchangeable proton resonances as indicators of imidazole hydrogen bonding in ferric cyanide complexes of heme peroxidases, Biochimica et Biophysica Acta (BBA) - Protein Structure and Molecular Enzymology 708, 317-325.

51. Satterlee, J. D., Erman, J. E., LaMar, G. N., Smith, K. M., and Langry, K. C. (1983) Assignment of hyperfine-shifted resonances in low-spin forms of cytochrome c peroxidase by reconstitutions with deuterated hemins, Journal of the American Chemical Society 105, 2099-2104.

52. Satterlee, J. D., Erman, J. E., and DeRopp, J. S. (1987) Proton hyperfine resonance assignments in cyanide-ligated cytochrome $\mathrm{c}$ peroxidase using the nuclear Overhauser effect, Journal of Biological Chemistry 262, 11578-11583.

53. Morita, Y., Yamashita, H., Mikami, B., Iwamoto, H., Aibara, S., Terada, M., and Minami, J. (1988) Purification, Crystallization, and Characterization of Peroxidase from Coprinus cinereus, Journal of Biochemistry 103, 693-699.

54. Shiro, Y., Kurono, M., and Morishima, I. (1986) Presence of endogenous calcium ion and its functional and structural regulation in horseradish peroxidase, Journal of Biological Chemistry 261, 9382-9390. 
55. Ogawa, S., Shiro, Y., and Morishima, I. (1979) Calcium binding by horseradish peroxidase $\mathrm{c}$ and the heme environmental structure, Biochemical and Biophysical Research Communications 90, 674-678.

56. De Ropp, J. S., Yu, L. P., and La Mar, G. N. (1991) 2D NMR of paramagnetic metalloenzymes: Cyanide-inhibited horseradish peroxidase, Journal of Biomolecular NMR 1, 175-190.

57. Satterlee, J. D., Erman, J. E., LaMar, G. N., Smith, K. M., and Langry, K. C. (1983) Assignment of hyperfine shifted resonances in high-spin forms of cytochrome c peroxidase by reconstitutions with deuterated hemins, Biochimica et Biophysica Acta (BBA) - Protein Structure and Molecular Enzymology 743, 246-255.

58. Thanabal, V., De Ropp, J. S., and La Mar, G. N. (1986) Determination of vinyl orientation in resting state and compound I of horseradish peroxidase by the proton nuclear Overhauser effect, Journal of the American Chemical Society 108, 4244-4246.

59. Thanabal, V., La Mar, G. N., and De Ropp, J. S. (1988) A nuclear Overhauser effect study of the heme crevice in the resting state and compound I of horseradish peroxidase: evidence for cation radical delocalization to the proximal histidine, Biochemistry 27, 5400-5407.

60. Neuhaus, D., and Williamson, M. P. (2000) The nuclear Overhauser effect in structural and conformational analysis, Wiley- $\mathrm{VCH}$.

61. Kalk, A., and Berendsen, H. J. C. (1976) Proton magnetic relaxation and spin diffusion in proteins, Journal of Magnetic Resonance (1969) 24, 343-366.

62. Guilard, R., Kadish, K. M., and Smith, K. M. (2000) The porphyrin handbook, Vol. 5, Academic Press.

63. Lee, K.-B., La Mar, G. N., Mansfield, K. E., Smith, K. M., Pochapsky, T. C., and Sligar, S. G. (1993) Interpretation of hyperfine shift patterns in ferricytochromes b5 in terms of angular position of the heme: a sensitive probe for peripheral heme protein interactions, Biochimica et Biophysica Acta (BBA) - Protein Structure and Molecular Enzymology 1202, 189-199.

64. Thanabal, V., and La Mar, G. N. (1989) A nuclear Overhauser effect investigation of the molecular and electronic structure of the heme crevice in lactoperoxidase, Biochemistry 28, 7038-7044.

65. Dugad, L. B., and Goff, H. M. (1992) Proton nuclear Overhauser effect study of the heme active site structure of Coprinus macrorhizus peroxidase, Biochimica et Biophysica Acta (BBA) - Protein Structure and Molecular Enzymology 1122, 63-69.

66. Chen, Z., de Ropp, J. S., Hernandez, G., and La Mar, G. N. (1994) 2D NMR Approaches to Characterizing the Molecular Structure and Dynamic Stability of the 
Active Site for Cyanide-Inhibited Horseradish Peroxidase, Journal of the American Chemical Society 116, 8772-8783.

67. Poulos, T. L. (2010) Thirty years of heme peroxidase structural biology, Archives of Biochemistry and Biophysics 500, 3-12.

68. Szigeti, K., Smeller, L., Osváth, S., Majer, Z., and Fidy, J. (2008) The structure of horseradish peroxidase $\mathrm{C}$ characterized as a molten globule state after $\mathrm{Ca} 2+$ depletion, Biochimica et Biophysica Acta (BBA) - Proteins \& Proteomics 1784, 1965-1974. 


\section{CHAPTER IV. ${ }^{1}$ H-NMR STUDY OF PARAMAGNETIC CYTOCHROME P450CAM-CYANIDE COMPLEXES: ASSIGNMENT OF HYPERFINE-SHIFTED HEME RESONANCES}

\subsection{Introduction}

Cytochrome P450 (CYP) enzymes are a widely distributed superfamily of hemecontaining, single chain monooxygenases involved in a diversity of vital processes. The P450 enzymes can catalyze the monooxygen insertion of a variety of hydrophobic compounds, such as steroids, fatty acids, and exogenous xenobiotics. All p450s share a common catalytically active species, compound I, which is formed by activating the heme-bound molecular oxygen through a heterolytic $\mathrm{O}-\mathrm{O}$ bond cleavage (1-2). In contrasting with most other hemoproteins that possess a proximal histidine ligand, the distinctive and highly conserved active site structure in all P450 enzymes utilizes a cysteine thiolate coordinating with the prosthetic heme as the proximal ligand (3).

In spite of the large amount of physicochemical data collected over the years about structure function relationships in P450s, detailed understanding of the effect of substrate binding on the changes of the protein structure are required. Particularly attention shall be put near the dioxygen binding site at the heme iron and in the I-helix groove, because of their suggested important role for oxygen activation (4). Understanding the mechanism and specificity of substrate binding in the cytochrome P450 superfamily is of great importance in explaining its key role in drug metabolism, toxicity, xenobiotic degradation, and several important biosynthetic pathways.

Among the various members of the P450 superfamily, cytochrome P450cam (EC 1.14.15.1; CYP 101; P450cam hereafter), from bacteria Pseudomonas putida, is the most 
extensively studied and best characterized CYP because of its high solubility, relative stability, and ease of purification. Especially, the mature recombinant enzyme production using Escherichia coli expression system makes P450cam more accessible for studies for most purposes. The natural function of P450cam is to catalyze the stereo- and regiospecifically hydroxylation of $(1 R)$-camphor. The heme prosthetic group of P450cam has been shown to adopt a conformation (Figure 4.1) (5) different from the heme in HRP (6) and SBP (7), but similar to that in $\mathrm{CPO}(8)$ and $\mathrm{CcP}(9)$.

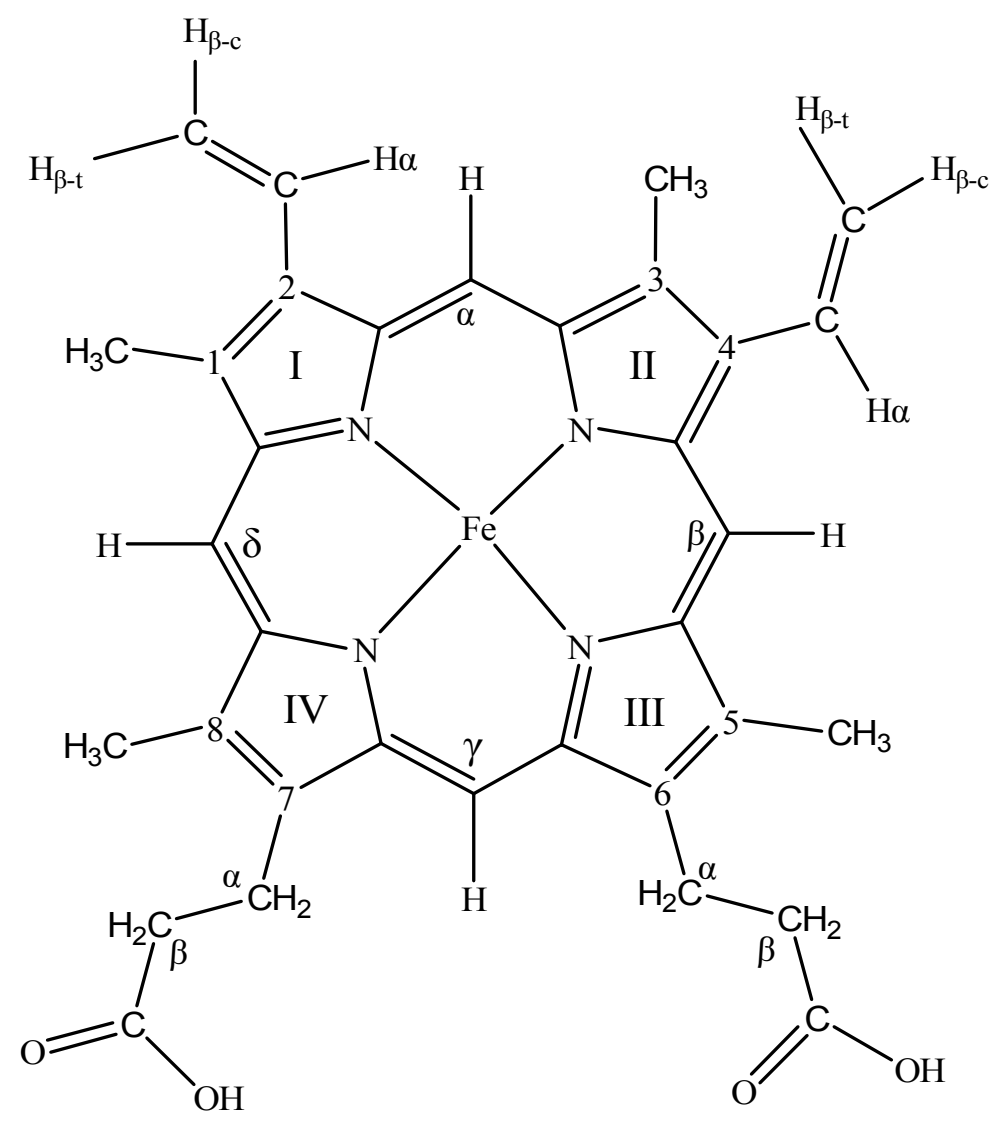

Figure 4.1 schematic presentations of structure and numbering system for iron protoporphyrin IX in P450cam with both the 2- and 4-vinyls in the cis orientation.

Among the numerous chemical and spectroscopic techniques that are used for the structural investigation of hemoproteins, NMR spectroscopy and x-ray crystallography 
have been proved as the most informative and authoritative methods for characterizing the structure of paramagnetic metalloenzymes. NMR spectroscopy, comparing with x-ray crystallography, is more emphasized on analyzing the active site heme cavity environment of hemoproteins in solution, which is more similar to the physiological conditions. Therefore, NMR spectroscopy can provide valuable complementary information to the solid state crystal structures and also enables the possibility of dynamic investigations of hemoprotein-substrates interactions.

Recently, x-ray crystallography has been employed in many studies of P450cam to illustrate enzyme conformation changes and functions of heme propionate groups (10-15). However, only a few controversial NMR studies of paramagnetic P450cam have been reported with only heme methyls assigned even with no reported progress after the year 2000 (16-21), in great contrast with the extensive and in depth NMR studies on heme peroxidases, such as $\mathrm{C} c \mathrm{P}(22-34), \operatorname{HRP}(6,23,35-51)$, and $\mathrm{CPO}$ (52-57). The stagnation in the paramagnetic P450cam NMR study is mainly caused by the proximal cysteine ligation imparted inefficiency of relaxation and magnetic properties to the heme iron electron, which adversely affect ${ }^{1} \mathrm{H}$ NMR spectra resulting short $\mathrm{T}_{1}$, large linewidths and small magnetic anisotropy (58). As a result it is more difficult to extract useful information from protons in proximity to the heme iron. Moreover, the lack of a characteristic structural model such as $\mathrm{C} c \mathrm{P}$ in peroxidase studies, the relative instability of P450cam compared to heme peroxidases, and the difficulty in the reconstitution of isotopic labeled heme, all exist as obstacles for detailed NMR studies of P450cam.

The urgent need of definitive resonance assignments of P450cam NMR spectrums, together with the successful application of the most powerful two-dimensional (2D) 
NMR techniques in characterizing the heme active site structure of chloroperoxidase, which is also a cysteine thiolate hemoprotein, triggered my ambition to use the twodimensional NMR methods to unambiguously assign the major hyperfine-shifted signals and consequently elucidate the active site structure of P450cam in solution. I present herein the results of a comprehensive NMR study of P450cam-cynaide complexes with and without substrate camphor presence, by utilizing a combination of 2D NMR methods and the Curie intercepts obtained from variable temperature experiments. The results lead to the unequivocal assignment of all heme hyperfine-shifted signals, together with certain correlated diamagnetic resonances. The resulting assigned hyperfine shift pattern yields a valuable insight into the heme cavity of P450cam and will facilitate future assignments of the substrate and amino acid side-chain signals of interest.

\subsection{Materials and Methods}

\subsubsection{Materials}

Escherichia coli Rosetta II (DE3) cells were from Invitrogen (Carlsbad, CA). Dithiothreitol (DTT), carbon monoxide, hydrogen peroxide, were purchased from SigmaAldrich (St. Louis, MO). DEAE sepharose fast flow and Sephadex G-75 were purchased from GE Healthcare (Piscataway, NJ). Water with $18.2 \mathrm{megohm} \cdot \mathrm{cm}$ resistivity were freshly produced from a Milli-Q BioCel Water Purification System (Millipore, Billerica, MA). All other reagents were of the highest purity available from Fisher Scientific (Pittsburg, PA). All chemicals were used without further purification.

\subsubsection{Preparation of Protein Samples}

The wild type (WT) P450cam protein were expressed in Escherichia coli strain Rosetta II (DE3) transformed with a plasmid constructed by ligation of pET-30a (+) with 
P450cam gene at the restriction sites of NdeI and HindIII. The bacteria were grown in the terrific broth media containing $100 \mathrm{mg} / \mathrm{L}$ of kanamycin at $37^{\circ} \mathrm{C}$ and induced with $1 \mathrm{mM}$ IPTG at $\mathrm{OD}_{600}$ over 0.8 . Induction was allowed to proceed at $30^{\circ} \mathrm{C}$ for another 18 hours prior to harvesting by high-speed centrifugation. Cell pellets were resespended and lysed at $4{ }^{\circ} \mathrm{C}$ for 30 minutes in $50 \mathrm{mM}$ Tris- $\mathrm{HCl}$ buffer at $\mathrm{pH} 7.4$ containing $50 \mathrm{mM} \mathrm{KCl}$, $0.5 \mathrm{mM}$ DTT, $1 \mathrm{mM}$ camphor, $1 \mathrm{mM}$ EDTA, $200 \mu \mathrm{M}$ PMSF, $40 \mathrm{U} / \mathrm{ml}$ Dnase I, $3 \mathrm{U} / \mathrm{ml}$ Rnase A, and $2 \mathrm{mg} / \mathrm{ml}$ lysozyme. The sonicated and filtrated cell lysate were then concentrated by ultrafiltration (Amicon cell) with a 30,000 Da cut-off membrane and purified by passage through a fast flow DEAE Sepharose column $(50 \mathrm{ml})$ equilibrated with $50 \mathrm{mM}$ Tris- $\mathrm{HCl}$ buffer ( $\mathrm{pH}$ 7.4). After the initial wash, the protein was eluted with a linear 0 to $0.5 \mathrm{M} \mathrm{KCl}$ gradient. The red fractions containing the $\sim 45 \mathrm{kDa}$ proteins were pooled for further purification by size exclusion gel filtration chromatography (Sephadex G-75, $500 \mathrm{ml}$ ) with $100 \mathrm{mM}$ potassium phosphate buffer at $\mathrm{pH}$ 7.4. All the steps in the protein purification were performed at $4{ }^{\circ} \mathrm{C}$. The purification steps typically yield pure P450cam protein with $\mathrm{Rz}$ values $\left(\mathrm{A}_{417} / \mathrm{A}_{280}\right)$ higher than 1.4. The concentration of the enzyme sample was determined using the Soret extinction coefficient $\varepsilon_{417}=115 \mathrm{mM}^{-1} \mathrm{~cm}^{-1}$ for the native low-spin camphor-free P450cam (59).

\subsubsection{NMR Spectroscopy}

Proton nuclear magnetic resonance $\left({ }^{1} \mathrm{H}\right.$ NMR) spectra for the native and cyanidebound proteins with or without excess camphor were recorded at $298 \mathrm{~K}$ on a Bruker Avance 600 (Ultrashield) spectrometer operating at a proton frequency of $599.93 \mathrm{MHz}$. Protein samples for NMR experiments were prepared in $\mathrm{D}_{2} \mathrm{O}$ buffer $(10 \mathrm{mM}$ phosphate, $\mathrm{pH}$ 7.4, uncorrected for isotope effect), by at least five times of isotope exchanges 
through a centrifuging microconcentrator (Centricon-30) at $4{ }^{\circ} \mathrm{C}$. All NMR samples contained more than $2 \mathrm{mM}$ purified P450cam and the cyanide-adducts of the protein were prepared by the addition of a 20 -fold molar excess of cyanide from a freshly made $1 \mathrm{M}$ stock solution of $\mathrm{KCN}$ in $99.9 \% \mathrm{D}_{2} \mathrm{O}$. The camphor bound protein samples were prepared by the addition of $1 \mathrm{M}$ camphor stock solution in deuterated methanol- $\mathrm{d}_{4}$ into the protein solutions until the final camphor concentration reached $7.8 \mathrm{mM}$.

The ${ }^{1} \mathrm{H}$ NMR spectra of the ferric low-spin forms (native P450cam without camphor bound, and camphor free and camphor bound P450cam cyanide-adducts) were obtained by collecting 1000-2000 scans with $4 \mathrm{~K}$ data points over a $30-40 \mathrm{kHz}$ bandwidth, and a repetition time of 0.2 seconds with solvent presaturation during relaxation delay. A 16-bit digitizer was used and the spectra were obtained by multiplication of the free induction decays with a 20 to $40 \mathrm{~Hz}$ apodization. Fast repetition spectra of the low-spin ferric protein forms were recorded with a $125 \mathrm{kHz}$ sweep width, 2048 data points, and a repetition rate of $10 \mathrm{~ms}$ to detect the fast relaxing proton signals which are intrinsically broad. The NMR spectrum of the high-spin ferric camphor-bound P450cam was collected with a $125 \mathrm{kHz}$ spectral width, 16384 data points, and a repetition rate of approximately 50 per second. A 12-bit digitizer was used and 20,000 scans were averaged. Free induction decays were exponentially multiplied by a line broadening factor of $100 \mathrm{~Hz}$ before Fourier transformation to improve the signal-to-noise ratio of the spectra. The residual solvent signal was suppressed with presaturation during relaxation delay. Chemical shift values were referenced to the residual HDO signal at $4.76 \mathrm{ppm}$.

The spin-lattice relaxation times $\left(T_{1}\right)$ of the hyperfine-shifted protons for the cyanide complexes of camphor free and camphor-bound P450cam were determined by a variation 
of the standard inversion-recovery sequence to include a composite $180^{\circ}$ pulse. A repetition rate of 3.3 per second and a $90^{\circ}$ transmitter pulse of $7.6 \mu$ s was used. The $T_{1}$ values were estimated from the null point with the relationship of $T_{1}=\tau / \ln 2$. The NOE experiments on the cyanide complexes of the protein were performed similar to what is described in Chapter 3, with the irradiation time of $100 \mathrm{~ms}$ and a weak decoupler power of $60 \mathrm{~dB}$.

Phase-sensitive NOESY spectra for the cyanide-bound derivatives of P450cam were acquired at $298 \mathrm{~K}$ with mixing times ranging from 15 to $20 \mathrm{~ms}$. Typical NOESY spectra were collected with 256 experiments in the F1 dimension. In general, 400 scans were accumulated for each F1 experiment, which was acquired with 4096 complex points in the F2 dimension over a spectral width of 45 or $65 \mathrm{ppm}$. The residual solvent signal in all NOESY experiments was suppressed using a 200-ms presaturation with a weak decoupler power. COSY spectra were collected with all parameters similar to NOESY.

Pulse sequences utilized in all the experiments employed single $90^{\circ}$ hard pulses to maximize the bandwidth of excitation. The Soret band at $440 \mathrm{~nm}$, which is characteristic for the P450cam/cyanide and P450cam/cyanide/camphor complexes, did not shift throughout all the NMR study.

\subsection{Results}

\subsubsection{Proton NMR of the Ferric Forms of the Native Low-Spin Camphor-Free P450cam and the Native High-Spin Camphor-Bound P450cam}

The proton NMR spectra of the ferric forms of P450cam (Figure 4.2) are essentially identical to the results reported previously $(17,19,21)$. Without the cyanide ligation, the NMR spectrum of the native ferric camphor-free P450cam (Figure 4.2A) exhibits a sixcoordinated low-spin feature with a water molecule serving as the distal ligand. The 
hyperfine-shifted $19.5 \mathrm{ppm}$ and $14.2 \mathrm{ppm}$ peaks had been assigned previously (19) to the 5- and 1-methyl groups of the prosthetic heme, respectively. A novel far upfield-shifted, fast relaxing broad resonance at $-21.7 \mathrm{ppm}$ (Figure 4.2A inset) is noticed for the first time. Based on the extremely short $\mathrm{T}_{1}$ of $\sim 1 \mathrm{~ms}$, the relaxation properties, the large contact shift, the expected distance to the heme iron center, and the similarity of such resonances from CPO (57), this resonance is assigned to one of the $\beta-\mathrm{CH}_{2}$ protons of Cys357, the heme iron proximal ligand. Unfortunately, this substrate-free resting state enzyme exhibits a relatively poor stability, which prevents the further application of extensive NMR study.

The native camphor-bound P450cam reveals a five-coordinated high-spin nature (Figure 4.2B) with the distal water expelled upon substrate binding. The resonances at $62.9,58.6,40.6$, and $36.4 \mathrm{ppm}$ had been tentatively assigned to the four heme methyl groups with the order $8>3>5>1$ (19), which is different from the previous proposed assignments with the order $5>1>8>3(21)$. The considerably broad resonances in the NMR spectrum can provide only limited information about the structural properties of the enzyme. Therefore, no further efforts were made to analyze the spectra of these resting states P450cam in this study.

\subsubsection{Proton NMR of the Ferric Low-Spin Cyanide-Adducts of Camphor-Free and Camphor-Bound P450cam}

The NMR spectral properties of the cyanide-bound, ferric low spin derivatives of camphor-free and camphor-bound P450cam have been focused on in this study, because their short electronic relaxation times and large magnetic anisotropy yield much sharper 

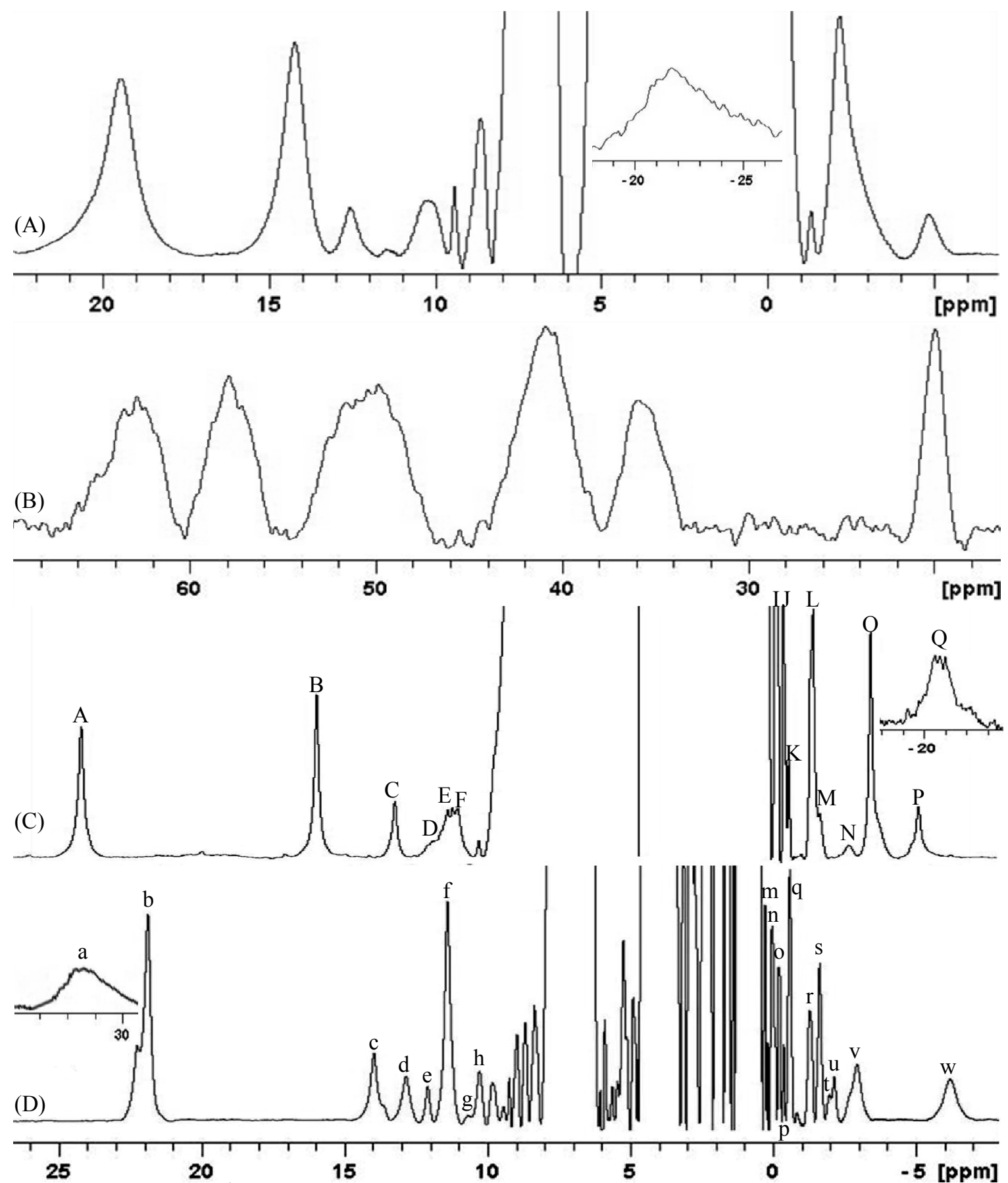

Figure 4.2 $600 \mathrm{MHz}{ }^{1} \mathrm{H}$ NMR spectra of the ferric forms of (A) low-spin resting state camphor-free P450cam in $\mathrm{D}_{2} \mathrm{O}$ and $100 \mathrm{mM}$ pH 7.0 phosphate buffer with the inset showing the $-21.7 \mathrm{ppm}$ peak, (B) high-spin camphor-bound $\mathrm{P} 450$ cam in $\mathrm{D}_{2} \mathrm{O}$ and $100 \mathrm{mM}$ pH 7.0 phosphate buffer containing $4 \mathrm{mM} \mathrm{d}$ camphor, (C) low spin cyanide complex of the camphor-free P450cam in $\mathrm{D}_{2} \mathrm{O}$ and $100 \mathrm{mM}$ phosphate buffer ( $\mathrm{pH}$ 7.0) with the inset showing the $-21.7 \mathrm{ppm}$ peak, and (D) low spin cyanide complex of the camphor-bound $\mathrm{P} 450 \mathrm{cam}$ in $\mathrm{D}_{2} \mathrm{O}$ and $100 \mathrm{mM} \mathrm{pH} 7.0$ phosphate buffer containing $7.8 \mathrm{mM}$ d-camphor at $298 \mathrm{~K}$ with the inset showing the $33.1 \mathrm{ppm}$ peak. 
and better resolved signals in their proton NMR spectra. Therefore, valuable information about the electronic and molecular structural properties of the heme pocket can be provided.

The proton NMR spectrum of the substrate-free P450cam- $\mathrm{CN}^{-}$complex in $\mathrm{D}_{2} \mathrm{O}$ solution is shown in Figure 4.2C. The paramagnetically shifted signals were observed with high similarity to that of other heme peroxidase cyanide derivatives $(57,60)$. The three intense hyperfine-shifted signals "A", "B", and "O" with integrated intensities of three protons each are typical of heme methyl groups. Such three methyl peak homologies have been tentatively assigned to the 5-, 1- and 3- heme methyls for the substrate-free P450cam-alkyl isocyanide complexes, respectively $(16,19)$, which is in contrast to the previously proposed assignments of the pyridine adduct (17) and the cyanide adduct (21). The newly discovered far upfield resonance in the native ferric camphor-free P450cam is also observed after cyanide binding as shown in the inset of Figure 4.2C labeled as peak "Q" at $-21.7 \mathrm{ppm}$ with very short $\mathrm{T}_{1}$ of $\sim 1 \mathrm{~ms}$. Accordingly, this peak "Q" is assigned to the Cys357 $\beta$-proton for the same reason as mentioned above.

The proton NMR spectrum of the camphor-bound $\mathrm{P} 450$ cam- $\mathrm{CN}^{-}$complex in $\mathrm{D}_{2} \mathrm{O}$ solution shown in Figure 4.2D reveals a far downfield-shifted broad peak "a", which is also strongly relaxed with a short $\mathrm{T}_{1}$ of $\sim 1 \mathrm{~ms}$. The single proton Peak "a" is previously assigned to a proximal Cys357 $\mathrm{C}_{\beta} \mathrm{H}$ in P450cam (21) and $\mathrm{CPO}$ (57). The upfield peak at $-21.7 \mathrm{ppm}$, which is observed in either native or cyanide-bound forms of camphor-free P450cam, is not detected with camphor presence, indicating a conformational change around the proximal cysteine upon substrate binding. The binding of camphor to P450cam-CN ${ }^{-}$complex results in 2-4 ppm low-field bias of the heme methyls (Figure 4.2 $\mathrm{C} \& \mathrm{D})$. The assignments of methyl peak "b" and "f" to $8-\mathrm{CH}_{3}$ and $3-\mathrm{CH}_{3}$ was suggested 
(21) on the basis of the tentative assignments in the pyridine complex (17). However, disagreement was proposed by Mouro et al., stating that all low-spin P450cam complexes should have the same chemical shift order of the heme methyl groups $5>1>8>3$ (19).

The assignment of the hyperfine-shifted signals for the camphor-free and camphorbound $\mathrm{P} 450 \mathrm{cam}-\mathrm{CN}^{-}$complexes was achieved through comparison with the cyanideadducts of CPO and other heme peroxidases $(23,31,34,40-41,57)$, in combination with the P450cam crystal structure analysis and the correlation information revealed by the NOESY and COSY spectrum. The chemical shifts and the corresponding diamagnetic shift values predicted from Curie plot intercept at $\mathrm{T}^{-1}=0$, as well as the spin-lattice relaxation times for the hyperfine-shifted resonances and their assignments in the ferric cyanide-adducts of camphor-free and camphor-bound P450cam are compiled in Table 4.1, along with the previously reported corresponding parameters in CPO (57).

The NOESY spectrum of the ferric camphor-free and camphor-bound $\mathrm{P} 450 \mathrm{cam}-\mathrm{CN}^{-}$ in $\mathrm{D}_{2} \mathrm{O}$ are shown in Figure 4.3 and 4.4, respectively. For the relatively simple camphorfree P450cam- $\mathrm{CN}^{-}$, unambiguous assignment of the hyperfine shifts is achieved based only on the well characterized NOESY spectrum. While for the more complicated camphor-bound P450cam-CN ${ }^{-}$spectrum, NOESY information combining with the 1D NOE experiments (Figure 4.5B-E), which have higher resolution in the paramagnetic region, as well as the scalar connectivities revealed by COSY (Figure 4.6) lead to the unambiguous assignment for most of the nonexchangeable hyperfine-shifted protons. Some resonances buried in the diamagnetic envelope complex region are also tentatively assigned in the 2D maps. The suggested assignments for hyperfine shifts are further verified by the Curie intercepts predicted diamagnetic shift of the resonances (Figure 4.7). 
Table 4.1 Proton NMR chemical shift (ppm), $T_{1}(\mathrm{~ms})$, and Curie plots intercept (ppm) values of the assigned resonances for the low-spin cyanide derivatives of camphor-free (- cam) and camphor-bound (+ cam) P450cam, and CPO ${ }^{\mathrm{a}}$.

\begin{tabular}{|c|c|c|c|c|c|c|c|c|c|c|}
\hline \multicolumn{3}{|c|}{$\mathrm{Fe}^{\mathrm{III}} \mathrm{P} 450 \mathrm{cam}-\mathrm{CN}^{-}(-\mathrm{cam})$} & \multicolumn{4}{|c|}{$\mathrm{Fe}^{\mathrm{III}} \mathrm{P} 450 \mathrm{cam}^{-\mathrm{CN}^{-}}(+\mathrm{cam})$} & \multicolumn{3}{|c|}{$\mathrm{CPO}^{-\mathrm{CN}^{-}}$} & \multirow[b]{2}{*}{ Assignment } \\
\hline Signal & Shift & $\mathrm{T}_{1}$ & Signal & Shift & $\mathrm{T}_{1}$ & Int & Shift & $\mathrm{T}_{1}$ & Int & \\
\hline \multirow[b]{2}{*}{ b } & ppm & $m s$ & & ppm & $m s$ & ppm & ppm & $m s$ & ppm & \\
\hline & --- & --- & $\mathrm{a}$ & 33.1 & 1 & -11.1 & 39.0 & 1.5 & -11.0 & Cys357 H $\beta$ \\
\hline A & 24.3 & 36 & $\mathrm{~b}$ & 22.0 & 40 & 6.5 & 24.0 & 33 & 0.1 & $5-\mathrm{CH}_{3}$ \\
\hline B & 16.1 & 34 & $\mathrm{f}$ & 11.4 & 40 & 7.8 & 20.7 & 30 & -0.7 & $1-\mathrm{CH}_{3}$ \\
\hline $\mathrm{C}$ & 13.3 & 31 & $\mathrm{c}$ & 14.0 & 36 & 7.5 & 14.8 & 29 & 8.7 & $2-\mathrm{H} \alpha$ \\
\hline $\mathrm{D}$ & 12.0 & 30 & $\mathrm{e}$ & 12.1 & 39 & 2.3 & --- & --- & --- & NA \\
\hline E & 11.5 & 16 & $\mathrm{~g}$ & 10.5 & 44 & 3.3 & --- & --- & --- & NA \\
\hline $\mathrm{F}$ & 11.1 & 33 & d & 12.9 & 38 & -2.8 & 14.1 & 35 & 3.3 & $6-\mathrm{H \alpha}$ \\
\hline G & 8.52 & --- & $\mathrm{h}$ & 10.3 & 43 & 7.0 & 11.6 & 30 & 3.5 & 6-Ha' \\
\hline c & 0.5 & --- & b & --- & --- & --- & 3 & --- & 4.2 & $4-\mathrm{H} \alpha$ \\
\hline c & 4.0 & --- & c & 4.4 & --- & --- & --- & --- & --- & $\alpha$-Meso \\
\hline c & 1.3 & --- & c & 1.4 & --- & --- & 5.8 & --- & 8.4 & $\beta$-Meso \\
\hline c & 7.5 & --- & c & 7.4 & --- & --- & --- & --- & --- & $\delta$-Meso \\
\hline b & --- & --- & c & 0.9 & --- & --- & 2.8 & --- & 2.6 & 6-H $\beta$ \\
\hline b & --- & --- & o & -0.2 & 86 & 1.3 & 2 & -- & 4.1 & 6-Hß' \\
\hline $\mathrm{I}$ & -0.2 & 35 & q & -0.6 & 38 & 2.2 & -2.7 & 70 & 9.7 & $2-\mathrm{H} \beta_{\text {trans }}$ \\
\hline $\mathrm{L}$ & -1.3 & 38 & $\mathrm{~s}$ & -1.6 & 40 & 0.7 & -4.4 & 80 & 10.8 & $2-\mathrm{H} \beta_{\mathrm{cis}}$ \\
\hline M & -1.6 & 35 & $\mathrm{p}$ & -0.3 & 40 & -0.2 & -5.2 & 58 & 4.6 & $4-\mathrm{H} \beta_{\text {cis }}$ \\
\hline $\mathrm{N}$ & -2.6 & --- & $\mathrm{r}$ & -1.3 & 250 & -0.6 & --- & --- & --- & NA \\
\hline $\mathrm{O}$ & -3.3 & 33 & c & 0.4 & --- & --- & --- & --- & --- & $3-\mathrm{CH}_{3}$ \\
\hline $\mathrm{P}$ & -5.0 & 38 & $\mathrm{u}$ & -2.1 & 43 & 3.3 & -1.4 & & 5.8 & $4-\mathrm{H} \beta_{\text {trans }}$ \\
\hline Q & -21.7 & 1 & $\mathrm{~b}$ & --- & --- & --- & -20.7 & 1.5 & 6.4 & Cys357 H $\beta^{\prime}$ \\
\hline b & --- & --- & $\mathrm{w}$ & -6.2 & 16 & 3.8 & --- & --- & --- & cam proton \\
\hline b & --- & --- & $\mathrm{c}$ & 2.3 & --- & --- & --- & --- & --- & cam proton \\
\hline
\end{tabular}

\footnotetext{
${ }^{a}$ Results for CPO-CN- are from ref (57) and the values from CPO assignment are symmetrically switched along the $\alpha-\gamma$ meso axis for comparison purpose. For example, the assigned $\mathrm{CPO} 8-\mathrm{CH}_{3}$ results are used to compare with the corresponding values of the $5-\mathrm{CH}_{3}$ in $\mathrm{P} 450 \mathrm{cam}$.

${ }^{\mathrm{b}}$ The assignment is not achieved or does not exist in this form.

${ }^{\mathrm{c}}$ The signal is located in the diamagnetic region and is not labeled with letter.
} 


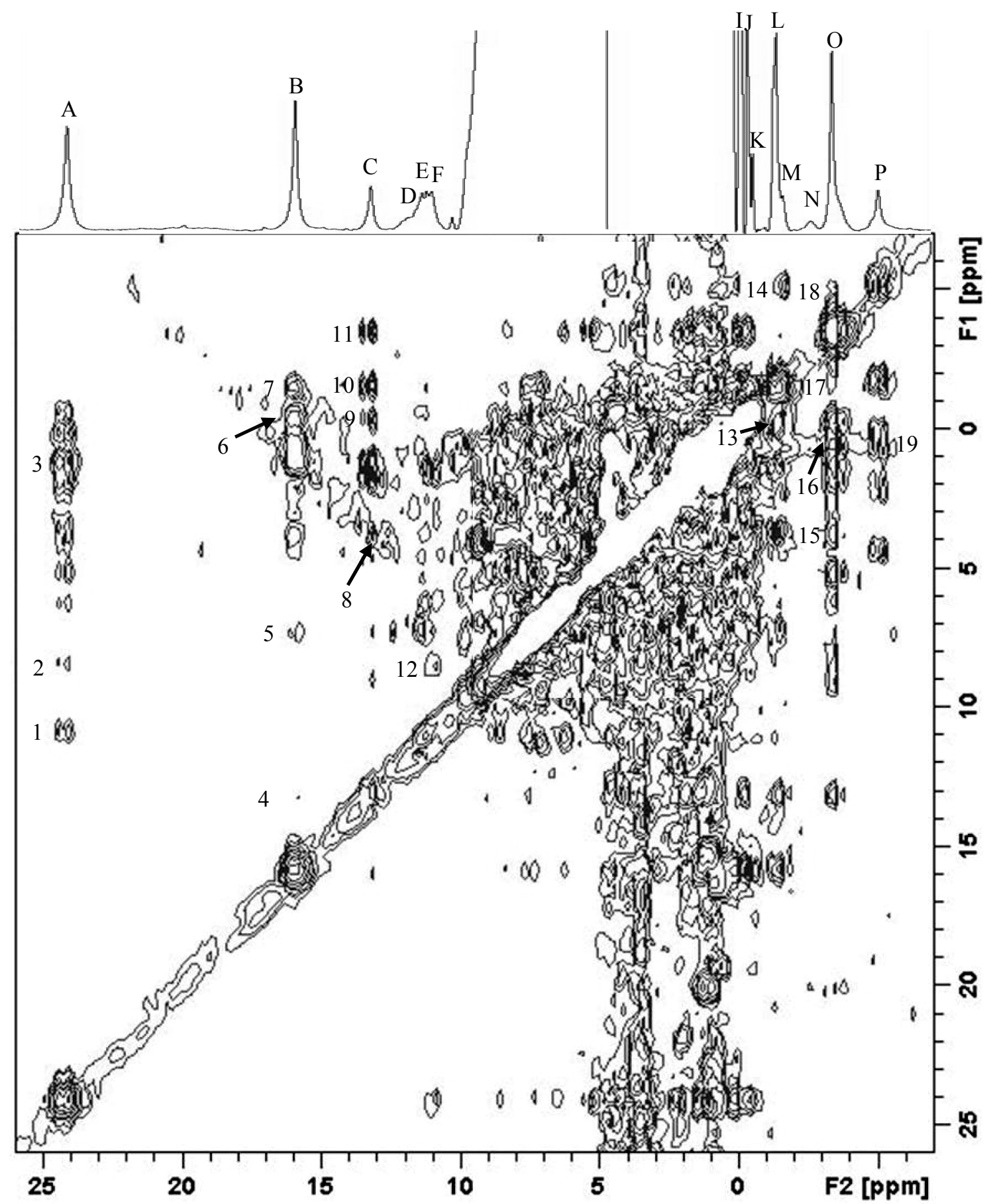

Figure 4.3 The $600 \mathrm{MHz}$ phase-sensitive ${ }^{1} \mathrm{H}$ NOESY spectra of $2.0 \mathrm{mM}$ substrate-free $\mathrm{P} 450 \mathrm{cam}-\mathrm{CN}^{-}$in $\mathrm{D}_{2} \mathrm{O}$ taken at $298 \mathrm{~K}$ with a mixing time of $15 \mathrm{~ms}$. Cross-peak assignments are as follows: $1,5-\mathrm{CH}_{3}: 6-\mathrm{H} \alpha ; 2$, $5-\mathrm{CH}_{3}: 6-\mathrm{H} \alpha ; 3,5-\mathrm{CH}_{3}: \mathrm{H}-\beta_{\text {meso }} ; 4,1-\mathrm{CH}_{3}: 2-\mathrm{H} \alpha ; 5,1-\mathrm{CH}_{3}: \mathrm{H}-\delta_{\text {meso }} ; 6,1-\mathrm{CH}_{3}: 2-\mathrm{H} \beta_{\text {trans }} ; 7,1-\mathrm{CH}_{3}: 2-\mathrm{H} \beta_{\text {cis }} ; 8$, 2-H $\alpha: \mathrm{H}-\alpha_{\text {meso }} ; 9,2-\mathrm{H} \alpha: 2-\mathrm{H} \beta_{\text {trans }} ; 10,2-\mathrm{H} \alpha: 2-\mathrm{H} \beta_{\text {cis }} ; 11,2-\mathrm{H} \alpha: 3-\mathrm{CH}_{3} ; 12,6-\mathrm{H} \alpha: 6-\mathrm{H} \alpha ; 13,2-\mathrm{H} \beta_{\text {cis }}: 2-\mathrm{H} \beta_{\text {trans }}$; $14,4-\mathrm{H} \beta_{\text {cis }}: 4-\mathrm{H} \beta_{\text {trans }} ; 15,3-\mathrm{CH}_{3}: \mathrm{H}-\alpha_{\text {meso }} ; 16,3-\mathrm{CH}_{3}: 4-\mathrm{H} \alpha ; 17,3-\mathrm{CH}_{3}: 4-\mathrm{H} \beta_{\text {cis }} ; 18,3-\mathrm{CH}_{3}: 4-\mathrm{H} \beta_{\text {trans }} ; 19,4-$ $\mathrm{H} \beta_{\text {trans }}: 4-\mathrm{H} \alpha$. 


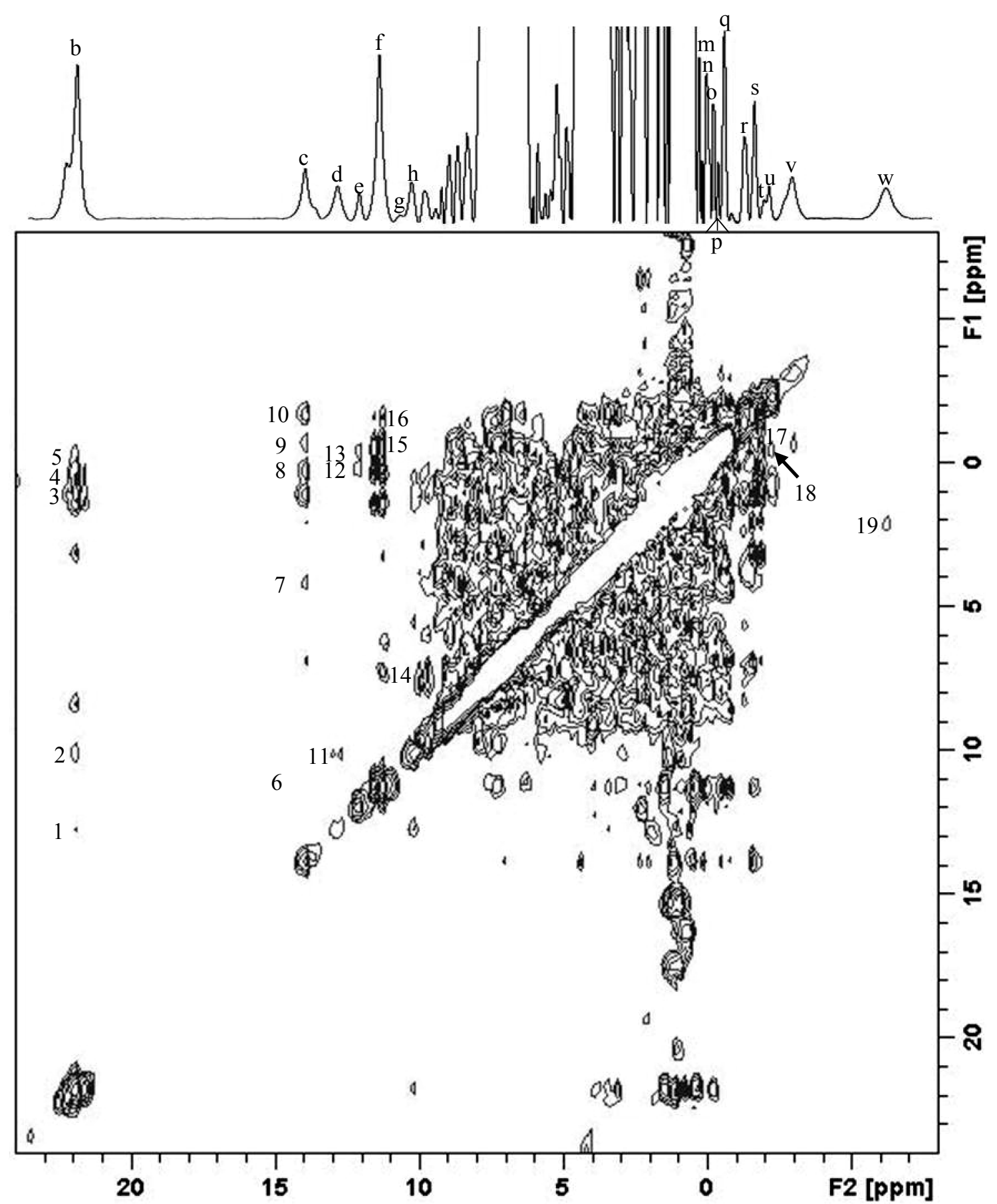

Figure 4.4 The $600 \mathrm{MHz}$ phase-sensitive ${ }^{1} \mathrm{H}$ NOESY spectra of $2.0 \mathrm{mM}$ camphor-bound $\mathrm{P} 450 \mathrm{cam}-\mathrm{CN}^{-}$in $\mathrm{D}_{2} \mathrm{O}$ taken at $298 \mathrm{~K}$ with a mixing time of $15 \mathrm{~ms}$. Cross-peak assignments are as follows: $1,5-\mathrm{CH}_{3}: 6-\mathrm{H} \alpha ; 2$,

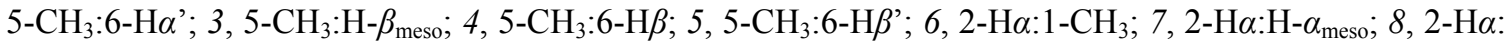
$3-\mathrm{CH}_{3} ; 9,2-\mathrm{H} \alpha: 2-\mathrm{H} \beta_{\text {trans }} ; 10,2-\mathrm{H} \alpha: 2-\mathrm{H} \beta_{\text {cis }} ; 11,6-\mathrm{H} \alpha: 6-\mathrm{H} \alpha ; 12,6-\mathrm{H} \alpha: 6-\mathrm{H} \beta ; 13,6-\mathrm{H} \alpha: 6-\mathrm{H} \beta$; $14,1-\mathrm{CH}_{3}: \mathrm{H}-$ $\delta_{\text {meso }} ; 15,1-\mathrm{CH}_{3}: 2-\mathrm{H} \beta_{\text {trans }} ; 16,1-\mathrm{CH}_{3}: 2-\mathrm{H} \beta_{\text {cis }} ; 17,2-\mathrm{H} \beta_{\text {cis }}: 2-\mathrm{H} \beta_{\text {trans }} ; 18,4-\mathrm{H} \beta_{\text {cis }}: 4-\mathrm{H} \beta_{\text {trans }} ; 19$, camphor proton: camphor proton. Cross-peak 6 can only be seen with lower contour levels. 


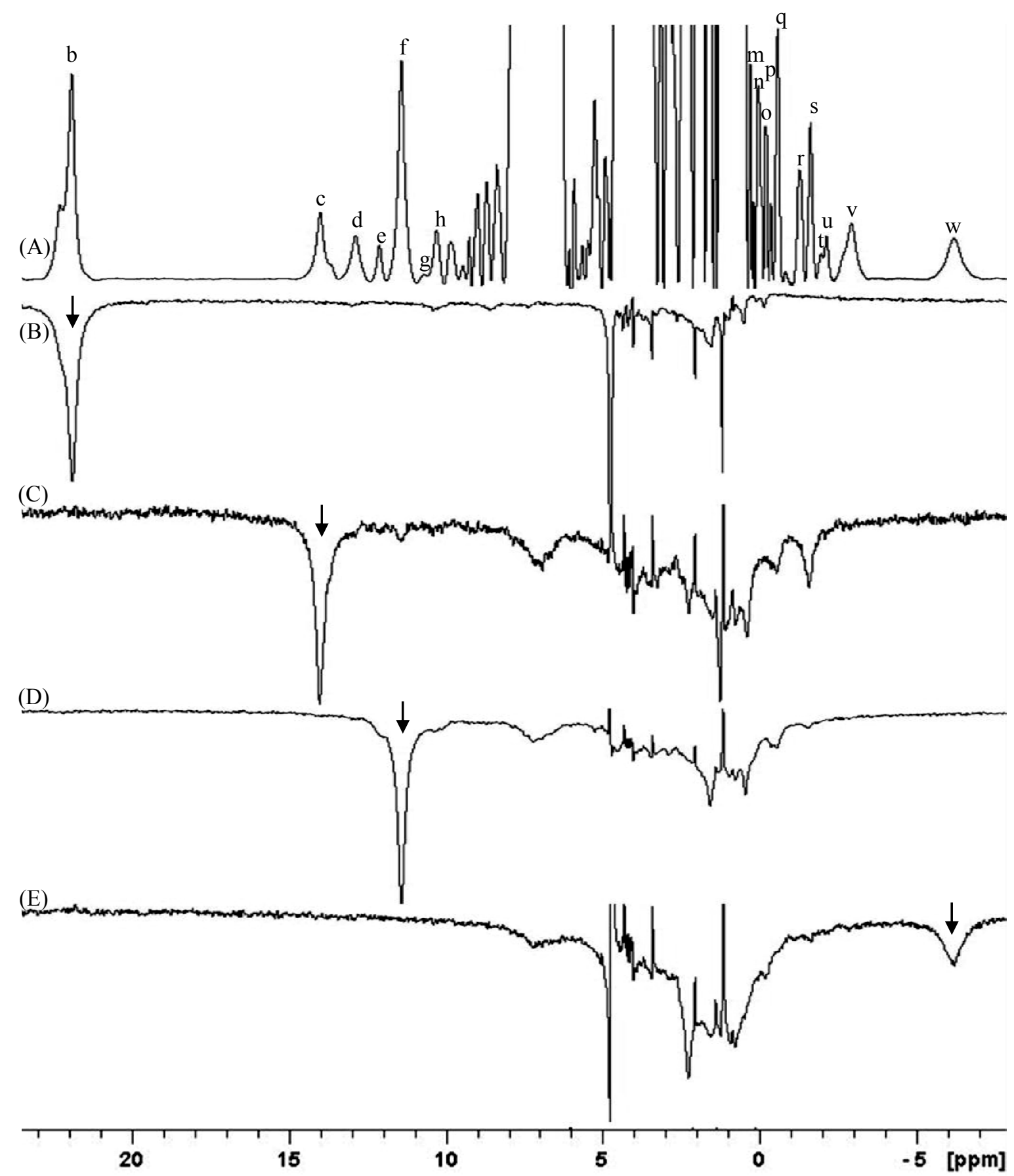

Figure 4.5 The 600-MHz proton NMR spectrum of (A) $2 \mathrm{mM}$ ferric low-spin camphor-bound form of P450cam- $\mathrm{CN}^{-}$in $\mathrm{D}_{2} \mathrm{O}$ and $100 \mathrm{mM} \mathrm{pH} 7.0$ phosphate buffer containing $7.8 \mathrm{mM}$ d-camphor at $298 \mathrm{~K}$. (B) trough (E) are the NOE difference spectra obtained upon irradiation of the hyperfine-shifted signals "b", "c", " $\mathrm{f}$ ", and "w", respectively. The irradiated peaks are indicated by a downward arrow in each difference spectrum. 


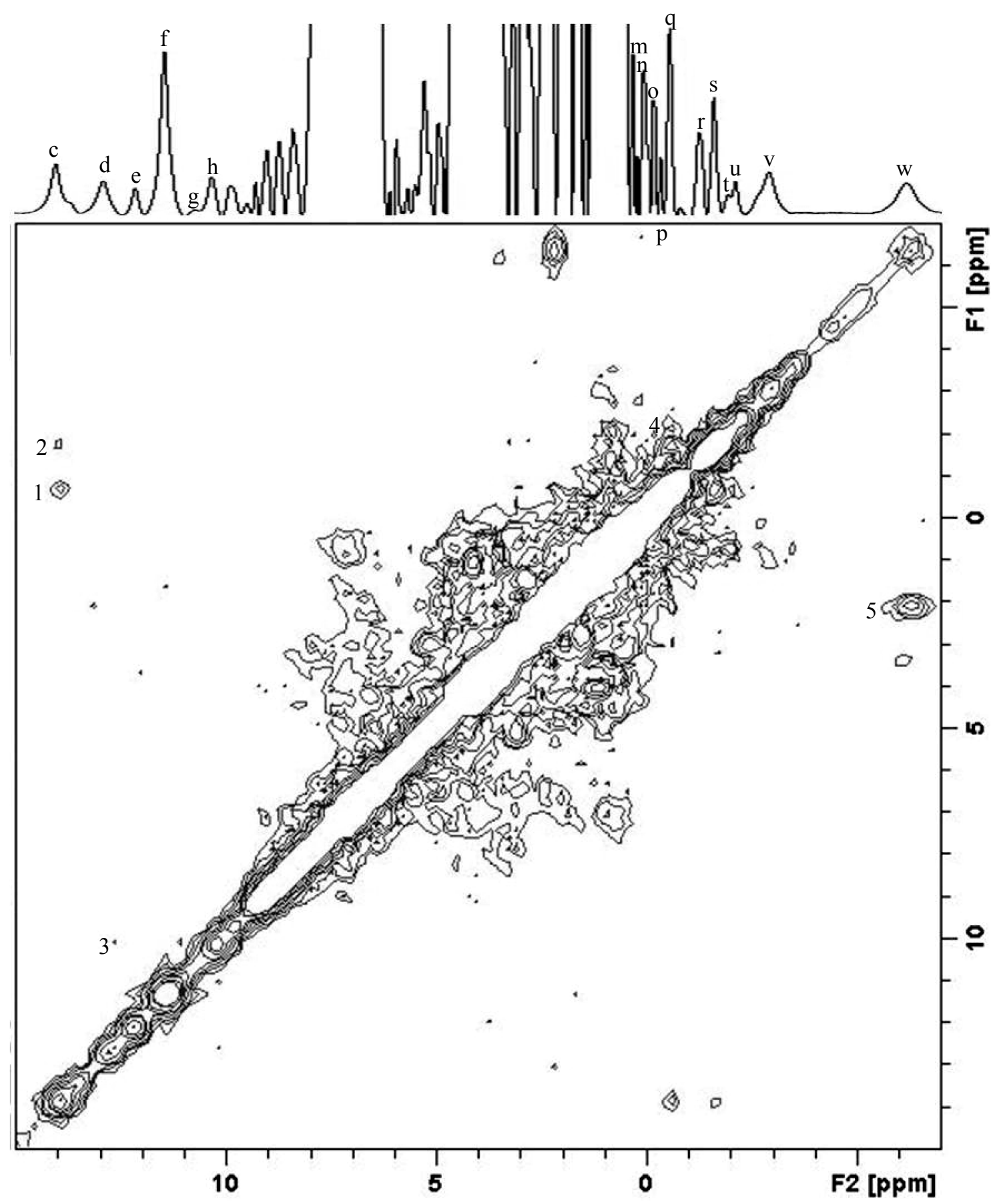

Figure 4.6 The $600 \mathrm{MHz}$ phase-sensitive ${ }^{1} \mathrm{H}$ COSY spectra of $2.0 \mathrm{mM}$ camphor-bound $\mathrm{P} 450 \mathrm{cam}-\mathrm{CN}^{-}$in $\mathrm{D}_{2} \mathrm{O}$ taken at $298 \mathrm{~K}$ after symmetrization for better resolution; note the different scale from that in the NOESY spectrum. Cross-peak assignments in complete agreement with previous NOESY assignments for hyperfine-shifted protons are as follows: $1,2-\mathrm{H} \alpha: 2-\mathrm{H} \beta_{\text {trans }} ; 2,2-\mathrm{H} \alpha: 2-\mathrm{H} \beta_{c i s} ; 3,6-\mathrm{H} \alpha: 6-\mathrm{H} \alpha$; 4 , 2- $\mathrm{H} \beta_{\text {trans }}: 2-$ $\mathrm{H} \beta_{c i s} ; 5$, camphor proton: camphor proton. 

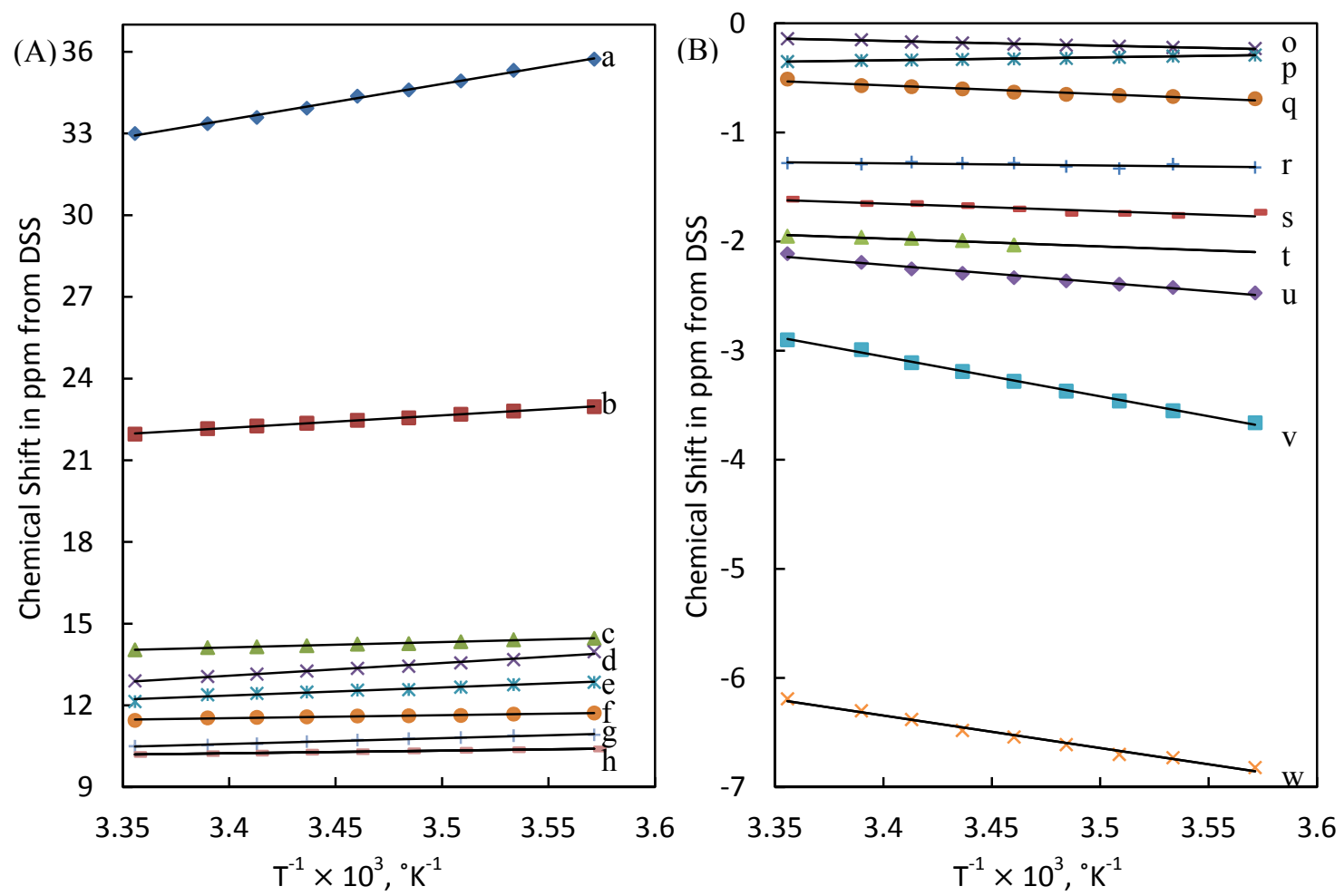

Figure 4.7 Curie plots of observed shift vs. reciprocal temperature for the resolved hyperfine-shifted resonances of camphor-bound $\mathrm{P} 450 \mathrm{cam}-\mathrm{CN}^{-}$in the (A) downfield and (B) upfield regions. The letter designation of each peak following the scheme of Figure 4.2D is given to the right of each line, and the extrapolated diamagnetic intercept at $\mathrm{T}^{-1}=0$ is given in Table 4.1 .

\subsection{Discussion}

\subsubsection{Assignment of the Hyperfine-Shifted Resonances for Ferric Camphor-Free P450cam-CN- Complex}

The ferric camphor-free $\mathrm{P} 450 \mathrm{cam}-\mathrm{CN}^{-}$complex shows a typical hemoprotein-cyanide adduct NMR spectrum (Figure 4.2C) with the two well characterized heme methyl groups located at the most downfield region. These two peaks " $\mathrm{A}$ " and "B", as discussed before, shall arise from either $5-\mathrm{CH}_{3}$ and $1-\mathrm{CH}_{3}$ or the $8-\mathrm{CH}_{3}$ and $3-\mathrm{CH}_{3}$ pair on heme depending on the position and orientation of the proximal ligand (61-62). This is confirmed by the NOESY connections as shown in Figure 4.3, in which peak "A" gives 
weak NOEs to peak "F" at $11.1 \mathrm{ppm}$ and peak "G" at $8.5 \mathrm{ppm}$, typical as the NOE pattern of a heme methyl near a propionate group as shown in many heme peroxidases such as HRP, CcP, and CPO $(28,32,34,44,57)$. The methyl peak "B", on the other hand, yields a very weak NOE to peak "C" at 13.3 ppm and two weak NOEs to the upfield peaks "I" at -0.2 ppm and "L" at -1.3 ppm. Clear NOEs are also observed between "C", "I" and "L", which is in perfect agreement with the three protons from a same heme vinyl group. Therefore, peak " $\mathrm{B}$ " must be either 1- or 3-methyl of the prosthetic heme.

Another methyl peak "O" in the upfield region at $-3.3 \mathrm{ppm}$ is observed and has been suggested to be the other heme methyl next to a vinyl group in contrast with the methyl peak " $\mathrm{B}$ ". This is confirmed by the weak NOEs between peak "O" and two peaks " $\mathrm{M}$ " at $-1.6 \mathrm{ppm}$ and "P" at $-5.0 \mathrm{ppm}$, two resonances located in the typical upfield region similar to heme vinyl- $\beta$ protons in other hemoproteins $(23,40,57)$. The last heme methyl resonance in pairing with peak "O" must be buried in the diamagnetic envelope, which is a common feature for most hemoproteins. Interestingly, a weak NOE is observed between peak "C" and peak "O", which lead to the solid conclusion that peak "O" must arise from the $3-\mathrm{CH}_{3}$ because peak " $\mathrm{C}$ " has been assigned to the proton from a vinyl group that is near to another heme methyl "B". Since both vinyl groups in P450cam heme adopt the cis orientation (Figure 4.1), peak "C" can be unambiguously assigned to the 2vinyl $\mathrm{H} \alpha$, and consequently, resonance " $\mathrm{B}$ " are assigned to the $1-\mathrm{CH}_{3}$, with the paring methyl " $A$ " assigned to the 5- $\mathrm{CH}_{3}$. Resonances " $F$ " and "G" therefore must arise from the 6-propinate $\alpha$-protons. The assignments of three heme methyl groups results a chemical shift order of $5>1>8>3$, in perfect agreement with the previous assignments using magnetization transfer method and isocyanides (19), and in disagreement with the earlier 
putative assignment with the order $8>3>5>1$, which was determined solely on homology with $\operatorname{HRP}(17,21)$.

The vinyl $\beta$-protons are assigned by comparing their relative NOE strengths to decide the cis- or trans- identity. For example, comparing with "L", resonance "I" reveals a much stronger NOE with the 1-CH3 peak "B", and a weaker NOE with peak "C", yielding the assignment of "L" to 2-vinyl $\mathrm{H} \beta$-cis and "I" to 2-vinyl $\mathrm{H} \beta$-trans. Similarly, the resonances " $\mathrm{M}$ " and "P" are assigned to 4-vinyl $\mathrm{H}_{\beta}$-cis and 4-vinyl $\mathrm{H}_{\beta}$-trans, respectively, and a peak at $0.5 \mathrm{ppm}$ showing NOE with both of the 4-H $\beta$ 's is assigned to the $4-\mathrm{H} \alpha$.

The heme $\alpha$-, $\beta$-, and $\delta$-mesoprotons are tentatively assigned by careful examination of the NOESY correlations with their geminal partners $\left(2-\mathrm{H} \alpha \& 3-\mathrm{CH}_{3}, 5-\mathrm{CH}_{3}\right.$, and 1$\mathrm{CH}_{3}$, respectively) within the proposed common mesoproton resonance range around 1-7 ppm (40).

\subsubsection{Assignment of the Hyperfine-Shifted Resonances for Ferric Camphor-bound P450cam-CN- Complex}

The binding of camphor results a more complicated spectrum (Figure 4.2D), with both the downfield methyl signals shifted by $2-4 \mathrm{ppm}$ to the low-field and the upfield methyl peak overwhelmed by the diamagnetic envelope, indicating an electronic field change in the heme cavity induced by the substrate binding.

The decided chemical shift order of heme methyl groups of $5>1>8>3$ was suggested to be consistent in all ferric low-spin P450cam forms (19). Therefore, methyl peaks "b" at $22.0 \mathrm{ppm}$ and "f" at $11.4 \mathrm{ppm}$ are assigned to the 5- and 1-methyl, respectively. The NOE correlations between peak " $b$ " and two downfield-shifted peaks 
"d" and "h" are consistent in both 1D and 2D NOE spectra (Figure $4.4 \& 4.5 \mathrm{~B}$ ), leading the assignment of "d" and "h" to the two 6-propionate $\alpha$-protons, which is confirmed by the COSY correlation between "d" and "h" (Figure 4.6). Furthermore, saturation of peak "b" yields detection of a peak at $0.9 \mathrm{ppm}$ and peak "o" at $-0.2 \mathrm{ppm}$ in the 1D NOE difference spectrum, which is also revealed in the NOESY spectrum with peak "d" showing the same NOE pattern. Because of the indicated geminal relationships between these resonances and the fact that propionate $\beta$-protons are normally located around the upfield region, the " 0.9 ppm" peak and peak "o" are assigned as the two 6-propionate $\beta$ protons.

The NOE pattern of the assigned 1- $\mathrm{CH}_{3}$ resonance " $\mathrm{f}$ " with a downfield-shifted peak "c" at $14.0 \mathrm{ppm}$ and two upfield-shifted peaks "q" at $-0.6 \mathrm{ppm}$ and "s" at $-1.6 \mathrm{ppm}$ are very similar to that in the camphor-free P450cam-CN". Correspondingly, peak "c" is assigned to the 2-vinyl $\mathrm{H} \alpha$ and signals "q" and "s" are assigned to 4-vinyl $\mathrm{H} \beta$-trans and 4-vinyl $\mathrm{H} \beta$-cis, respectively. These assignments are solid proved by the COSY result, and therefore, the early proposed putative assignment of peak "S" to an amino acid methyl group (21) must be misleaded by an overlapping at this position.

The $3-\mathrm{CH}_{3}$ is identified from the diamagnetic envelope through careful examination of both 1D and 2D NOE experiments. Similar NOE correlations between 2-Ha and a peak at $0.4 \mathrm{ppm}$ as in the camphor-free $\mathrm{P} 450 \mathrm{cam}-\mathrm{CN}^{-}$leads to the assignment of the 3methyl protons. Assignment of the $\beta$-protons from the 4-vinyl group is achieved by revealing the geminal relationships between peaks "p" at $-0.3 \mathrm{ppm}$ and " $\mathrm{u}$ " at $-2.1 \mathrm{ppm}$ from NOESY spectrum without confirmation from the COSY, which is because of the relatively low resolution in that area. The heme $\alpha$-, $\beta$-, and $\delta$-mesoprotons are assigned 
again within the 1-7 ppm region with information from NOESY correlations with their geminal heme resonances. The assigned resonances are closely located to the previously assigned mesoproton resonances in the camphor-free P450cam cyanide derivative, suggesting the success of these assignments.

The most upfield shifted resonances "w" displays no correlation with any paramagnetic resonance, indicating it is not derived from any heme proton. Nonetheless, the short $\mathrm{T}_{1}(16 \mathrm{~ms})$ reveals that it is in proximity to the heme iron, suggesting its origin from the proton of the substrate camphor, which is located closely to the heme iron in the distal side. The intensive cross peak between the peak "W" and a resonance at $2.3 \mathrm{ppm}$ shown in the COSY map (Figure 4.6) reveals the position of another camphor proton. Further verification of this assignment can be accomplished by using deuterated camphor as substrate.

The unassigned downfield peak "e" and the 1-methyl resonance "f" share several common NOE correlations with peaks in the diamagnetic region, indication that peak "e" may arise from an amino acid near the 1-methyl group.

\subsubsection{The Heme thiolate ligand Cys357}

The very broad, rapid relaxing, strongly hyperfine-shifted peaks "a" and "Q" at 33.1 and -21.7 ppm share very similar chemical shift value, relaxation time and Curie intercept with the CPO-CN ${ }^{-}$hyperfine-shifted resonances at $39.0 \mathrm{ppm}$ and $-20.7 \mathrm{ppm}$, which have been firmly assigned to the $\beta-\mathrm{CH}_{2}$ protons of the coordinated proximal cysteine (57). However, in contrast to in $\mathrm{CPO}$, the two resonances in P450cam can not coexist at the same time unless in a mixture of camphor-bound and camphor-free forms of P450cam$\mathrm{CN}^{-}$. The resonance "a" are only detected in the camphor-bound ferric $\mathrm{P} 450 \mathrm{cam}-\mathrm{CN}^{-}$ 
complex, while resonance "Q" exists in both native and cyanide derivative of camphorfree P450cam, indicating a distance and orientation change upon camphor binding. This camphor induced conformational change is proved by the distances measured from the crystal structure of camphor-free native P450cam and camphor-bound $\mathrm{P} 450 \mathrm{cam}-\mathrm{CN}^{-}$ (Table 4.2). It is shown that the distances between both the $\mathrm{Cys} 357 \beta-\mathrm{CH}_{2}$ protons and the heme iron increase by $\sim 0.3 \AA$ upon camphor binding. Additionally, the camphor bound induces one of the Cys $\beta-\mathrm{CH}_{2}$ protons shifting toward the 5-methyl group but away from the $\gamma$-meso direction. Meanwhile the other $\mathrm{Cys} \beta-\mathrm{CH}_{2}$ proton shifts away from both the 5-methyl and the $\gamma$-meso directions, indicating an orientation twist of the proximal Cys induced by camphor. The distances of the $\mathrm{Cys} \beta-\mathrm{CH}_{2}$ protons to the heme iron in $\mathrm{CPO}$ is right in between of the distances in camphor-free and camphor-bound P450cam. This may explain why both of the $\mathrm{Cys} \beta-\mathrm{CH}_{2}$ proton resonances can emerge at the same time in $\mathrm{CPO}-\mathrm{CN}^{-}$.

Table 4.2 Distances between proximal Cys $\beta$-protons and selected atoms in the low-spin cyanide adducts of camphor-free (- cam) and camphor-bound (+ cam) P450cam, and CPO.

\begin{tabular}{ccc|cc|cc}
\hline & \multicolumn{2}{c|}{$(-\mathrm{cam}) \mathrm{P} 450 \mathrm{cam}^{\mathrm{a}}$} & \multicolumn{2}{c|}{$(+\mathrm{cam}) \mathrm{P} 450 \mathrm{cam}_{-} \mathrm{CN}^{-\mathrm{b}}$} & \multicolumn{2}{c}{$\mathrm{CPO}^{2} \mathrm{CN}^{-\mathrm{c}}$} \\
\cline { 2 - 7 } Proximal Cys & $\mathrm{H} \beta$ & $\mathrm{H} \beta^{\prime}$ & $\mathrm{H} \beta$ & $\mathrm{H} \beta^{\prime}$ & $\mathrm{H} \beta$ & $\mathrm{H} \beta^{\prime}$ \\
\hline Heme iron & 2.99 & 4.06 & 3.29 & 4.34 & 3.19 & 4.24 \\
5 - $\mathrm{CH}_{3}$ carbon & 4.37 & 5.58 & 4.28 & 5.84 & 4.27 & 5.68 \\
$\gamma$-meso proton & 3.72 & 5.25 & 4.42 & 5.74 & 4.46 & 5.95 \\
\hline
\end{tabular}

\footnotetext{
${ }^{a}$ Distances are measured from the crystal data (PDB code 1PHC).

${ }^{\mathrm{b}}$ Distances are measured from the crystal data (PDB code 1O76).

${ }^{\mathrm{c}}$ Distances are measured from the crystal data (PDB code 2CIY).
} 
In summary, the presented results provide the first unambiguous assignments for most of the hyperfine-shifted resonances of the ferric low-spin camphor-free and camphorbound $\mathrm{P} 450 \mathrm{cam}-\mathrm{CN}^{-}$complexes. These assignments are achieved mainly through $2 \mathrm{D}$ NMR methods with the careful analysis of the correlation information in combination with the well established and accepted assignment strategy from earlier hemoprotein NMR studies. The observed methyl order and NOE pattern are consistent with the prosthetic heme structure and previously proposed assignments. Some diamagnetic heme resonances are tentatively assigned according to the NOE correlations and assignments in other hemoproteins. The alternation of the novel proximal cysteine $\beta-\mathrm{CH}_{2}$ resonances induced by camphor binding is an indication of the conformational change near the proximal side. The presented work solved the trouble that had hindered the ${ }^{1} \mathrm{H}-\mathrm{NMR}$ study of P450cam for a long time, and can shed more light on future NMR studies of other cytochromes P450 and P450 mutants.

\subsection{References}

1. Dawson, J. H., and Sono, M. (1987) Cytochrome P-450 and chloroperoxidase: thiolate-ligated heme enzymes. Spectroscopic determination of their active-site structures and mechanistic implications of thiolate ligation, Chemical Reviews 87, 1255-1276.

2. Sono, M., Roach, M. P., Coulter, E. D., and Dawson, J. H. (1996) HemeContaining Oxygenases, Chemical Reviews 96, 2841-2888.

3. Cramer, S. P., Dawson, J. H., Hodgson, K. O., and Hager, L. P. (1978) Studies of the ferric forms of cytochrome P-450 and chloroperoxidase by extended x-ray absotption fine structure. Characterization of the iron-nitrogen and iron-sulfur distances, Journal of the American Chemical Society 100, 7282-7290.

4. Hui Bon Hoa, G., Helms, V., Wade, R. C., and Jung, C. (1996) Structural Changes in Cytochrome P-450cam Effected by the Binding of the Enantiomers (1R)-Camphor and (1S)-Camphor, Biochemistry 35, 14127-14138. 
5. Poulos, T. L., Finzel, B. C., and Howard, A. J. (1987) High-resolution crystal structure of cytochrome P450cam, Journal of Molecular Biology 195, 687-700.

6. Thanabal, V., De Ropp, J. S., and La Mar, G. N. (1986) Determination of vinyl orientation in resting state and compound I of horseradish peroxidase by the proton nuclear Overhauser effect, Journal of the American Chemical Society 108, 4244-4246.

7. Henriksen, A., Mirza, O., Indiani, C., Teilum, K., Smulevich, G., Welinder, K. G., and Gajhede, M. (2001) Structure of soybean seed coat peroxidase: A plant peroxidase with unusual stability and haem-apoprotein interactions, Protein Science 10, 108-115.

8. Sundaramoorthy, M., Terner, J., and Poulos, T. L. (1995) The crystal structure of chloroperoxidase: a heme peroxidase cytochrome P450 functional hybrid, Structure (London, England : 1993) 3, 1367-1378.

9. Poulos, T. L., Freer, S. T., Alden, R. A., Edwards, S. L., Skogland, U., Takio, K., Eriksson, B., Xuong, N., Yonetani, T., and Kraut, J. (1980) The crystal structure of cytochrome c peroxidase, Journal of Biological Chemistry 255, 575-580.

10. Aldag, C., Gromov, I. A., García-Rubio, I., von Koenig, K., Schlichting, I., Jaun, B., and Hilvert, D. (2009) Probing the role of the proximal heme ligand in cytochrome P450cam by recombinant incorporation of selenocysteine, Proceedings of the National Academy of Sciences 106, 5481-5486.

11. Asciutto, E. K., Dang, M., Pochapsky, S. S., Madura, J. D., and Pochapsky, T. C. (2011) Experimentally Restrained Molecular Dynamics Simulations for Characterizing the Open States of Cytochrome P450cam, Biochemistry.

12. Harada, K., Sakurai, K., Ikemura, K., Ogura, T., Hirota, S., Shimada, H., and Hayashi, T. (2008) Evaluation of the Functional Role of the Heme-6-propionate Side Chain in Cytochrome P450cam, Journal of the American Chemical Society 130, 432-433.

13. Hayashi, T., Harada, K., Sakurai, K., Shimada, H., and Hirota, S. (2009) A Role of the Heme-7-Propionate Side Chain in Cytochrome P450cam as a Gate for Regulating the Access of Water Molecules to the Substrate-Binding Site, Journal of the American Chemical Society 131, 1398-1400.

14. Lee, Y.-T., Wilson, R. F., Rupniewski, I., and Goodin, D. B. (2010) P450cam Visits an Open Conformation in the Absence of Substrate, Biochemistry 49, 34123419 . 
15. Sakurai, K., Shimada, H., Hayashi, T., and Tsukihara, T. (2009) Substrate binding induces structural changes in cytochrome P450cam, Acta Crystallographica Section F 65, 80-83.

16. Mouro, C., Bondon, A., Jung, C., Hui Bon Hoa, G., De Certaines, J. D., Spencer, R. G. S., and Simonneaux, G. (1999) Proton nuclear magnetic resonance study of the binary complex of cytochrome P450cam and putidaredoxin: interaction and electron transfer rate analysis, FEBS letters 455, 302-306.

17. Banci, L., Bertini, I., Marconi, S., Pierattelli, R., and Sligar, S. G. (1994) Cytochrome P450 and Aromatic Bases: A 1H NMR Study, Journal of the American Chemical Society 116, 4866-4873.

18. Keller, R. M., Wüthrich, K., and Debrunner, P. G. (1972) Proton Magnetic Resonance Reveals High-Spin Iron (II) in Ferrous Cytochrome P450cam from Pseudomonas putida, Proceedings of the National Academy of Sciences of the United States of America 69, 2073-2075.

19. Mouro, C., Bondon, A., Jung, C., De Certaines, J. D., and Simonneaux, G. (2000) Assignment of heme methyl 1H-NMR resonances of high-spin and low-spin ferric complexes of cytochrome P450cam using one-dimensional and two-dimensional paramagnetic signals enhancement (PASE) magnetization transfer experiments, European Journal of Biochemistry 267, 216-221.

20. Philson, S. B., Debrunner, P. G., Schmidt, P. G., and Gunsalus, I. C. (1979) The effect of cytochrome P-450cam on the NMR relaxation rate of water protons, Journal of Biological Chemistry 254, 10173-10179.

21. Wakasugi, K., Ishimori, K., and Morishima, I. (1996) NMR studies of recombinant cytochrome P450cam mutants, Biochimie 78, 763-770.

22. Alam, S. L., Satterlee, J. D., Mauro, J. M., Poulos, T. L., and Erman, J. E. (1995) Proton NMR Studies of Cytochrome c Peroxidase Mutant N82A: Hyperfine Resonance Assignments, Identification of Two Interconverting Enzyme Species, Quantitating the Rate of Interconversion, and Determination of Equilibrium Constants, Biochemistry 34, 15496-15503.

23. Banci, L., Bertini, I., Turano, P., Ferrer, J. C., and Mauk, A. G. (1991) Comparative proton NMR study of ferric low-spin cytochrome c peroxidase and horseradish peroxidase, Inorganic Chemistry 30, 4510-4516.

24. Bashir, Q., Volkov, A. N., Ullmann, G. M., and Ubbink, M. (2009) Visualization of the Encounter Ensemble of the Transient Electron Transfer Complex of Cytochrome $\mathrm{c}$ and Cytochrome c Peroxidase, Journal of the American Chemical Society 132, 241-247. 
25. Crowley, P. B., Brett, K., and Muldoon, J. (2008) NMR Spectroscopy Reveals Cytochrome c-Poly(ethylene glycol) Interactions, ChemBioChem 9, 685-688.

26. Nonaka, D., Wariishi, H., Welinder, K. G., and Fujii, H. (2009) Paramagnetic 13C and 15N NMR Analyses of the Push and Pull Effects in Cytochrome c Peroxidase and Coprinus cinereus Peroxidase Variants: Functional Roles of Highly Conserved Amino Acids around Heme, Biochemistry 49, 49-57.

27. Satterlee, J. D., Alam, S. L., Mauro, J. M., Erman, J. E., and Poulos, T. L. (1994) The Effect of the Asn82 $\rightarrow$ Asp Mutation in Yeast Cytochrome c Peroxidase Studied by Proton NMR Spectroscopy, European Journal of Biochemistry 224, 81-87.

28. Satterlee, J. D., and Erman, J. E. (1991) Proton NMR assignments of heme contacts and catalytically implicated amino acids in cyanide-ligated cytochrome $\mathrm{c}$ peroxidase determined from one-and two-dimensional nuclear Overhauser effects, Biochemistry 30, 4398-4405.

29. Satterlee, J. D., Erman, J. E., and DeRopp, J. S. (1987) Proton hyperfine resonance assignments in cyanide-ligated cytochrome c peroxidase using the nuclear Overhauser effect, Journal of Biological Chemistry 262, 11578-11583.

30. Satterlee, J. D., Erman, J. E., Mauro, J. M., and Kraut, J. (1990) Comparative proton NMR analysis of wild-type cytochrome c peroxidase from yeast, the recombinant enzyme from Escherichia coli, and an Asp-235 ---Asn-235 mutant, Biochemistry 29, 8797-8804.

31. Satterlee, J. D., Russell, D. J., and Erman, J. E. (1991) Proton homonuclear correlated spectroscopy as an assignment tool for hyperfine-shifted resonances in medium-sized paramagnetic proteins: cyanide-ligated yeast cytochrome c peroxidase as an example, Biochemistry 30, 9072-9077.

32. Savenkova, M. I., Satterlee, J. D., Erman, J. E., Siems, W. F., and Helms, G. L. (2001) Expression, Purification, Characterization, and NMR Studies of Highly Deuterated Recombinant Cytochrome c Peroxidase $\uparrow$, Biochemistry 40, 1212312131.

33. Volkov, A. N., Worrall, J. A. R., Holtzmann, E., and Ubbink, M. (2006) Solution structure and dynamics of the complex between cytochrome $\mathrm{c}$ and cytochrome $\mathrm{c}$ peroxidase determined by paramagnetic NMR, Proceedings of the National Academy of Sciences 103, 18945-18950.

34. Wang, X., and Lu, Y. (1999) Proton NMR Investigation of the Heme Active Site Structure of an Engineered Cytochrome c Peroxidase that Mimics Manganese Peroxidase $\dagger$, Biochemistry 38, 9146-9157. 
35. La Mar, G. N., and De Ropp, J. S. (1980) Proton nuclear magnetic resonance characterization of the electronic structure of horseradish peroxidase compound I, Journal of the American Chemical Society 102, 395-397.

36. La Mar, G. N., De Ropp, J. S., Smith, K. M., and Langry, K. C. (1980) Proton NMR characterization of heme rotational disorder in reconstituted horseradish peroxidase, Journal of the American Chemical Society 102, 4833-4835.

37. La Mar, G. N., and De Ropp, J. S. (1982) Proton NMR characterization of the state of protonation of the axial imidazole in reduced horseradish peroxidase, Journal of the American Chemical Society 104, 5203-5206.

38. Gonzalez-Vergara, E., Meyer, M., and Goff, H. M. (1985) Proton nuclear magnetic resonance spectroscopy of horseradish peroxidase isoenzymes: correlation of distinctive spectra with isoenzyme specific activities, Biochemistry 24, 6561-6567.

39. Thanabal, V., De Ropp, J. S., and La Mar, G. N. (1987) Identification of the catalytically important amino acid residue resonances in ferric low-spin horseradish peroxidase with nuclear Overhauser effect measurements, Journal of the American Chemical Society 109, 7516-7525.

40. Thanabal, V., DeRopp, J. S., and La Mar, G. N. (1987) Proton NMR study of the electronic and molecular structure of the heme cavity in horseradish peroxidase. Complete heme resonance assignments based on saturation transfer and nuclear Overhauser effects, Journal of the American Chemical Society 109, 265-272.

41. Thanabal, V., De Ropp, J. S., and La Mar, G. N. (1988) Proton NMR characterization of the catalytically relevant proximal and distal hydrogenbonding networks in ligated resting state horseradish peroxidase, Journal of the American Chemical Society 110, 3027-3035.

42. Thanabal, V., La Mar, G. N., and De Ropp, J. S. (1988) A nuclear Overhauser effect study of the heme crevice in the resting state and compound I of horseradish peroxidase: evidence for cation radical delocalization to the proximal histidine, Biochemistry 27, 5400-5407.

43. La Mar, G. N., Thanabal, V., Johnson, R. D., Smith, K. M., and Parish, D. W. (1989) Deuterium NMR study of structural and dynamic properties of horseradish peroxidase, Journal of Biological Chemistry 264, 5428-5434.

44. De Ropp, J. S., and La Mar, G. N. (1991) 2D NMR assignment of hyperfineshifted resonances in strongly paramagnetic metalloproteins: resting-state horseradish peroxidase, Journal of the American Chemical Society 113, 43484350 . 
45. Chen, Z., de Ropp, J. S., Hernandez, G., and La Mar, G. N. (1994) 2D NMR Approaches to Characterizing the Molecular Structure and Dynamic Stability of the Active Site for Cyanide-Inhibited Horseradish Peroxidase, Journal of the American Chemical Society 116, 8772-8783.

46. de Ropp, J. S., Chen, Z., and La Mar, G. N. (1995) Identification of Residues in the Aromatic Substrate Binding Site of Horseradish Peroxidase by $1 \mathrm{H}$ NMR Studies on Isoenzymes, Biochemistry 34, 13477-13484.

47. La Mar, G. N., Chen, Z., Vyas, K., and McPherson, A. D. (1995) An Interpretive Basis of the Hyperfine Shifts in Cyanide-Inhibited Horseradish Peroxidase Based on the Magnetic Axes and Ligand Tilt. Influence of Substrate Binding and Extensions to Other Peroxidases, Journal of the American Chemical Society 117, 411-419.

48. de Ropp, J. S., Mandal, P. K., and La Mar, G. N. (1998) Solution 1H NMR Investigation of the Heme Cavity and Substrate Binding Site in Cyanide-Inhibited Horseradish Peroxidase $\dagger$, Biochemistry 38, 1077-1086.

49. Asokan, A., de Ropp, J. S., Newmyer, S. L., Ortiz de Montellano, P. R., and La Mar, G. N. (2001) Solution 1H NMR of the Molecular and Electronic Structure of the Heme Cavity and Substrate Binding Pocket of High-Spin Ferric Horseradish Peroxidase: Effect of His42Ala Mutation, Journal of the American Chemical Society 123, 4243-4254.

50. de Ropp, J. S., Sham, S., Asokan, A., Newmyer, S., Ortiz de Montellano, P. R., and La Mar, G. N. (2002) Influence of the Distal His in Imparting Imidazolate Character to the Proximal His in Heme Peroxidase: 1H NMR Spectroscopic Study of Cyanide-Inhibited His $42 \rightarrow$ Ala Horseradish Peroxidase, Journal of the American Chemical Society 124, 11029-11037.

51. Nonaka, D., Wariishi, H., and Fujii, H. (2009) Paramagnetic 13C and 15N NMR Analyses of Cyanide- (13C15N-) Ligated Ferric Peroxidases: The Push Effect, Not Pull Effect, Modulates the Compound I Formation Rate $\dagger$, Biochemistry 48, 898-905.

52. Dugad, L. B., Wang, X., Wang, C. C., Lukat, G. S., and Goff, H. M. (1992) Proton nuclear Overhauser effect study of the heme active site structure of chloroperoxidase, Biochemistry 31, 1651-1655.

53. Goff, H. M., Gonzalez-Vergara, E., and Bird, M. R. (1985) High-resolution proton nuclear magnetic resonance spectroscopy of chloride peroxidase: identification of new forms of the enzyme, Biochemistry 24, 1007-1013. 
54. Lukat, G. S., and Goff, H. M. (1986) Proton and nitrogen-15 NMR spectroscopic studies of hydrogen ion-dependent pseudo-halide ion binding to chloroperoxidase, Journal of Biological Chemistry 261, 16528-16534.

55. Lukat, G. S., and Goff, H. M. (1990) A nuclear magnetic resonance study of axial ligation for the reduced states of chloroperoxidase, cytochrome P-450cam, and porphinatoiron(II) thiolate complexes, Biochimica et Biophysica Acta (BBA) Protein Structure and Molecular Enzymology 1037, 351-359.

56. Wang, X., and Goff, H. M. (1997) A nuclear paramagnetic relaxation study of the interaction of the cyclopentanedione substrate with chloroperoxidase, Biochimica et Biophysica Acta (BBA) - Protein Structure and Molecular Enzymology 1339, 88-96.

57. Wang, X., Tachikawa, H., Yi, X., Manoj, K. M., and Hager, L. P. (2003) Twodimensional NMR Study of the Heme Active Site Structure of Chloroperoxidase, Journal of Biological Chemistry 278, 7765-7774.

58. La Mar, G. N., Satterlee, J. D., and De Ropp, J. S. (2000) Nuclear Magnetic resonance of hemoproteins, In The porphyrin handbook (Guilard, R., Kadish, K. M., and Smith, K. M., Eds.), pp 188-299, Academic Press.

59. Dawson, J. H., Andersson, L. A., and Sono, M. (1982) Spectroscopic investigations of ferric cytochrome P-450-CAM ligand complexes. Identification of the ligand trans to cysteinate in the native enzyme, Journal of Biological Chemistry 257, 3606-3617.

60. Bertini, I., Turano, P., and Vila, A. J. (1993) Nuclear magnetic resonance of paramagnetic metalloproteins, Chemical Reviews 93, 2833-2932.

61. Guilard, R., Kadish, K. M., and Smith, K. M. (2000) The porphyrin handbook, Vol. 5, Academic Press.

62. Lee, K.-B., La Mar, G. N., Mansfield, K. E., Smith, K. M., Pochapsky, T. C., and Sligar, S. G. (1993) Interpretation of hyperfine shift patterns in ferricytochromes b5 in terms of angular position of the heme: a sensitive probe for peripheral heme protein interactions, Biochimica et Biophysica Acta (BBA) - Protein Structure and Molecular Enzymology 1202, 189-199. 


\section{CHAPTER V. CONCLUDING REMARKS}

In this dissertation I have investigated the native or modified active site structure of three hemoproteins with one- and two-dimensional NMR applications. The fundamental claim I have made in this dissertation is that, NMR techniques are very powerful for the structural elucidation of large organic molecule and hemoproteins.

In Chapter 2, the $\mathrm{N}$-alkylated heme of the allylbenzene inactivated chloroperoxidase was studied, in which the modified heme was extracted, demetallated and characterized by UV-vis, ES-MS/MS, and extensive 1D and 2D NMR methods. The results firmly established the structure of the modified heme as a $\mathrm{N}_{\mathrm{III}}$-(3-phenyl-2-hydroxyalkyl) ironporphyrin IX, which indicate that the allylbenzene induced CPO inactivation is highly regio- and stereospecific. The result of this study solved a long-lasting mystery and greatly enhanced our understanding about the active site environment and catalytic mechanism of CPO. Based on the combination of our results with the observation in CPO crystal structures, a new mechanism of $\mathrm{CPO}$ catalysis and topology of heme active site was proposed. In this mechanism, the phenyl groups of Phe103-Phe186 located at the end of CPO substrate accessing channel function as a clap and play important role in controlling the substrate binding position and orientation. This first-time proposed double-phenyl motif can well explain many catalytic activities displayed by CPO, such as regiospecific epoxidation and the formation of green $\mathrm{CPO}$ and its auto-restoration. Although more evidence is required to corroborate this novel motif, it extends and inspires our understanding of enzyme substrate binding strategy.

The biggest unresolved question from this study is that why the green heme monomer- $\mathrm{CN}^{-}$complex does not show any correlation information in all NMR spectra, 
such as COSY, NOESY, and ROESY. On the other hand, the green CPO shows no paramagnetically shifted signal, which cannot be explained by the formation of the green heme dimer within the protein. Another task is to further verify the proposed doublephenyl clamp mechanism, which could be explored by determining the crystal structure of CPO complexed with phenyl substrates. Site-specific mutation of the Phe103 and Phe186, and the application of theoretical calculations can also corroborate the elucidation of this mechanism. The future study of the mechanism-based inactivation of CPO can also include the study of allyacetate inactivated green CPO, because allyacetate has no phenyl group and therefore may reveal different binding strategy and $\mathrm{N}$-alkylation preference to the $\mathrm{CPO}$ active site.

In Chapter 3, the active site structure of SBP was characterized by one- and twodimensional NMR spectroscopic studies. All hyperfine shifted resonances from the heme prosthetic group and catalytically important amino acid residues including both proximal histidine that serves as the heme iron ligand and distal histidine that serves as the general acid-base catalyst during formation of compound I of SBP have been unambiguously assigned through (a) measurement of NOE connectivities, $(b)$ prediction of the Curie intercepts from both one- and two-dimensional variable temperature studies, $(c)$ comparison with assignments made for cyanide derivatives of several well characterized heme proteins such as cytochrome $c$ peroxidase and horseradish peroxidase, and $(d)$ examination of the crystal structural parameters of recombinant SBP. The NMR parameters and peak patterns of SBP are compared with those of HRP and $\mathrm{CcP}$. Our study indicates that results from NMR investigations of the protein in solution can complement and verify the results revealed by x-ray diffraction studies of the crystal 
form and thus provide a complete and better understanding of the actual structure of the protein. The similar NMR spectral patterns of SBP and HRP suggest very similar overall structural features of the heme cavity and similar activity of these two plant peroxidases. However, differences between the NMR property of the two proteins do exist and suggest structural differences of the two proteins are localized around the 2- and 4- vinyl groups of the heme, which may be responsible for the different activity and stability of the two proteins. No significant spectral changes were observed upon removal of calcium from SBP, indicating that calcium ions play similar structural and functional roles in these two peroxidases.

The next step in this study is to identify signals from all residues in the heme, as well as proximal and distal, environment, and eventually all residues in the heme periphery. Further assignments can be extended beyond those residues in immediate contact with the heme and also include all catalytically relevant residues in the proximal and distal environments. The resulted information from such study can further clarify the high stability and activity of SBP.

In Chapter 4, the first attempt for the assignments of most hyperfine-shifted resonances and some diamagnetic heme resonances of camphor-free and camphor-bound P450cam- $\mathrm{CN}^{-}$complexes are successfully achieved through 2D NMR applications. The observed heme methyl chemical shifts order of $5>1>8>3$ and the NOE pattern are consistent with the prosthetic heme structure and finalized the controversial literature results. Resonances from the two $\beta-\mathrm{CH}_{2}$ of the proximal cysteine are observed for the first time. Our results revealed that an alternation of the proximal cysteine $\beta-\mathrm{CH}_{2}$ resonances could be induced by camphor binding, which indicated a conformational 
change near the proximal side upon camphor binding.

Future work for this study is to assign more resonances from the prosthetic heme and residues around the heme cavity, and especially, from the substrate camphor. The kinetic NMR study of camphor binding is also a challenging but exciting task to solve the frustrating question about how camphor gets into the binding pocket. The NMR study of various P450cam mutants can also shed light on explaining the function of the mutated residues. 


\section{APPENDIX A \\ Sequences of Chloroperoxidase}

\section{General information}

The wild-type chloroperoxidase protein consists of 299 amino acids as listed in Figure A.

Figure A. Amino acid sequence of full-length of WT Chloroperoxidase.

1 XEPGSGIGYP YDNNTLPYVA PGPTDSRAPC PALNALANHG YIPHDGRAIS

51 RETLQNAFLN HMGIANSVIE LALTNAFVVC EYVTGSDCGD SLVNLTLLAE

101 PHAFEHDHSF SRKDYKQGVA NSNDFIDNRN FDAETFQTSL DVVAGKTHFD

151 YADMNEIRLQ RESLSNELDF PGWFTESKPI QNVESGFIFA LVSDFNLPDN

201 DENPLVRIDW WKYWFTNESF PYHLGWHPPS PAREIEFVTS ASSAVLAASV

251 TSTPSSLPSG AIGPGAEAVP LSFASTMTPF LLATNAPYYA QDPTLGPND 


\section{APPENDIX B}

\section{Sequences of Soybean Peroxidase}

\section{General information}

The wild-type soybean peroxidase protein consists of 304 amino acids as listed in Figure B.

Figure B. Amino acid sequence of full-length of WT Soybean Peroxidase.

1 QLTPTFYRET CPNLFPIVFG VIFDASFTDP RIGASLMRLH FHDCFVQGCD 51 GSVLLNNTDT IESEQDALPN INSIRGLDVV NDIKTAVENS CPDTVSCADI 101 LAIAAEIASV LGGGPGWPVP LGRRDSLTAN RTLANQNLPA PFFNLTQLKA 151 SFAVQGLNTL DLVTLSGGHT FGRARCSTFI NRLYNFSNTG NPDPTLNTTY 201 LEVLRARCPQ NATGDNLTNL DLSTPDQFDN RYYSNLLQLN GLLQSDQELF 251 STPGADTIPI VNSFSSNQNT FFSNFRVSMI KMGNIGVLTG DEGEIRLQCN $301 \mathrm{FVNG}$ 


\section{APPENDIX C}

\section{Sequences of Cytochrome P450cam}

\section{General information}

The wild-type cytochrome P450cam protein consists of 414 amino acids as listed in Figure C.

Figure C. Amino acid sequence of full-length of WT Cytochrome P450cam.

1 MTTETIQSNA NLAPLPPHVP EHLVFDFDMY NPSNLSAGVQ EAWAVLQESN

51 VPDLVWTRCN GGHWIATRGQ LIREAYEDYR HFSSECPFIP REAGEAYDFI

101 PTSMDPPEQR QFRALANQVV GMPVVDKLEN RIQELACSLI ESLRPQGQCN 151 FTEDYAEPFP IRIFMLLAGL PEEDIPHLKY LTDQMTRPDG SMTFAEAKEA 201 LYDYLIPIIE QRRQKPGTDA ISIVANGQVN GRPITSDEAK RMCGLLLVGG 251 LDTVVNFLSF SMEFLAKSPE HRQELIERPE RIPAACEELL RRFSLVADGR 301 ILTSDYEFHG VQLKKGDQIL LPQMLSGLDE RENACPMHVD FSRQKVSHTT 351 FGHGSHLCLG QHLARREIIV TLKEWLTRIP DFSIAPGAQI QHKSGIVSGV 401 QALPLVWDPA TTKAV 
VITA

ZHONGHUA WANG

Mar 01, 1981

1999-2003

2011
Born, Heilongjiang, China

Bachelor of Science, Life Sciences

Peking University, China

Doctoral Candidate in Chemistry Florida International University

Miami, Florida

\section{PUBLICATIONS AND PRESENTATIONS}

Wang, Zhonghua; Bian, Hedong; Wang, Xiaotang. Isolation and structure determination of the modified heme in allylbenzene-inactivated chloroperoxidase. Abstracts of Papers, 238th ACS National Meeting, Washington, DC, United States, August 16-20, 2009

Tian, Hui; Wang, Zhonghua; Terentis, Andrew C.; Wang, Xiaotang. Relative stability of iron-sulfur bond vs. iron-nitrogen bond in heme thiolate protein: Evidence from an engineered cytochrome P450cam with a novel heme iron ligand set. Abstracts of Papers, 238th ACS National Meeting, Washington, DC, United States, August 16-20, 2009

Tian, Hui; Wang, Zhonghua; Wang, Xiaotang. Single amino acid switch turns P450cam from a monooxygenase to an efficient peroxidase. Abstracts of Papers, 237th ACS National Meeting, Salt Lake City, UT, United States, March 22-26, 2009 